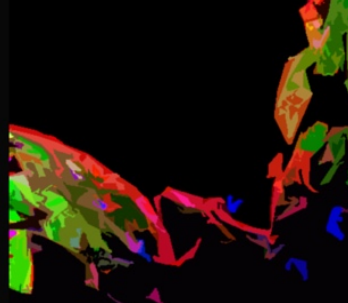

Universidad Autónoma de Madrid

\title{
Facultad de Ciencias
}

Departámento de Biología Molecular

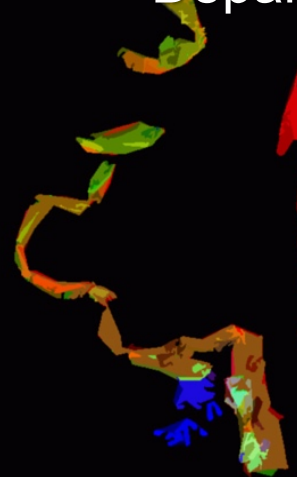

Study of Mikine-a and

(4)

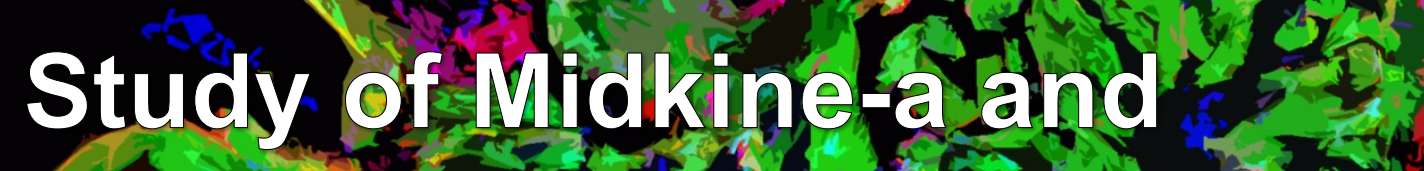
Caveolin-1 in zebrafish heart tin

- regeneration

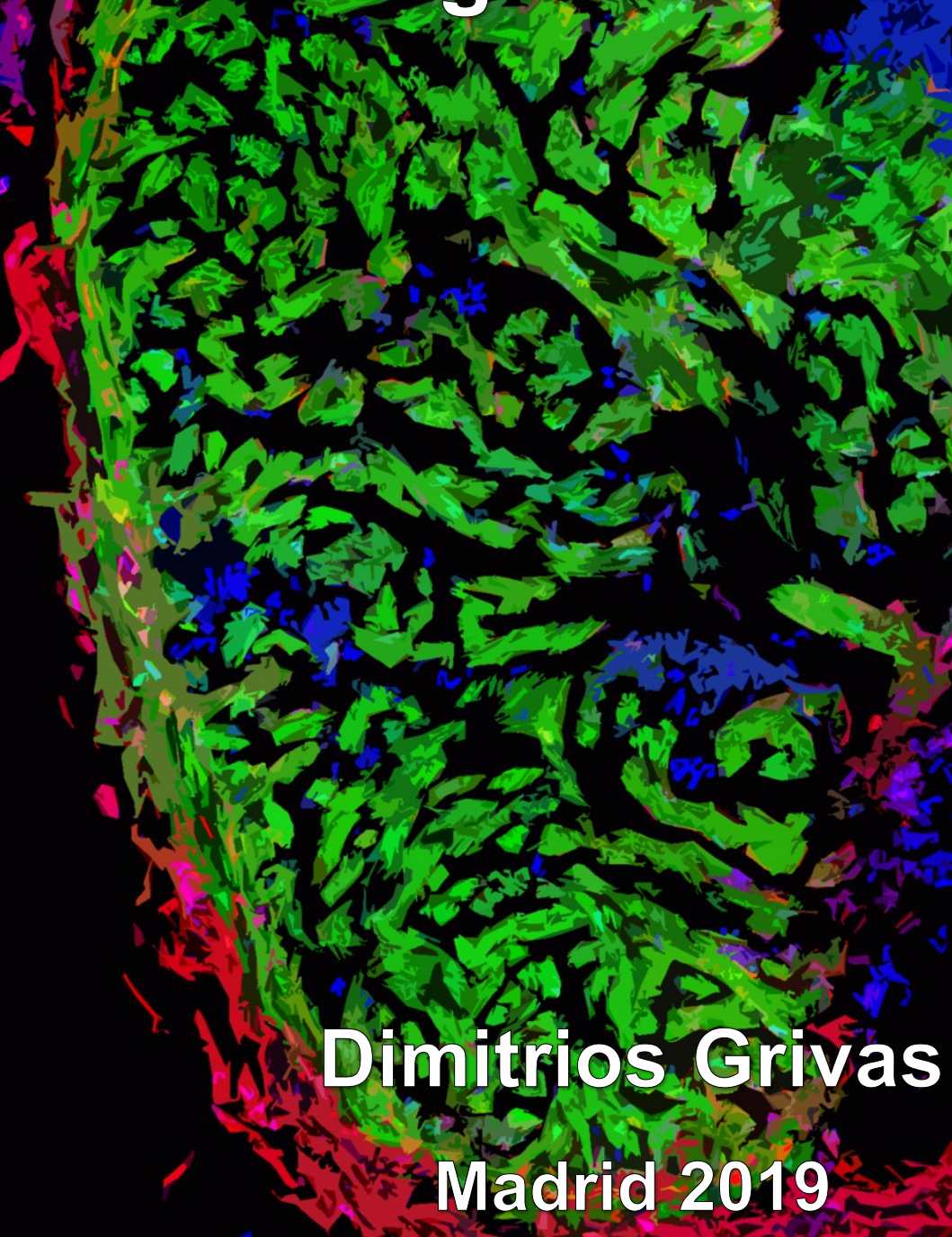



Universidad Autónoma de Madrid

Facultad de Ciencias

Departamento de Biología Molecular

Doctoral Thesis

\section{Study of Midkine-a and Caveolin-1 in zebrafish heart regeneration}

\section{Dimitrios Grivas}

Graduated in Biology

Director: Dr. José Luis de la Pompa Mínguez

Centro Nacional de Investigaciones Cardiovasculares (CNIC) 


\section{Cover legend}

Front cover image: $7 \mathrm{dpci} \operatorname{Tg}(m y l 7: G F P)$ heart labelled for Cav1(red).

Back cover image: $7 \mathrm{dpci} \operatorname{Tg}(m y l 7: G F P)$ heart labelled for Mdka (red). 


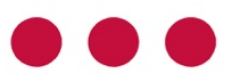

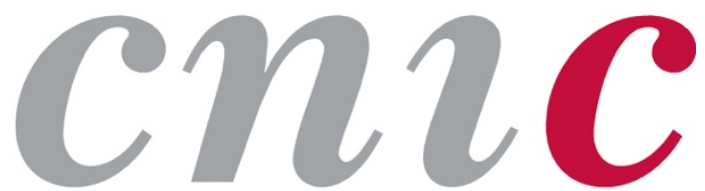

Dr. José Luis de la Pompa Mínguez, head of the Intercellular Signalling in Cardiovascular Development \& Disease Lab, Centro Nacional de Investigaciones Cardiovasculares Carlos III (CNIC),

\section{CERTIFIES:}

That Mr. Dimitrios Grivas, Graduate in Biology by the University of Athens, Greece, has completed her Doctoral Thesis entitled "Study of Midkine-a and Caveolin-1 in zebrafish heart regeneration" under my supervision and meets the necessary requirements to obtain the $\mathrm{PhD}$ degree in Molecular Biosciences. To this purpose, he will defend her doctoral thesis at the Universidad Autónoma de Madrid. I hereby authorize its defense in front of the appropriate Thesis evaluation tribunal.

I issue this certificate in Madrid on October $17^{\text {th }}, 2019$.

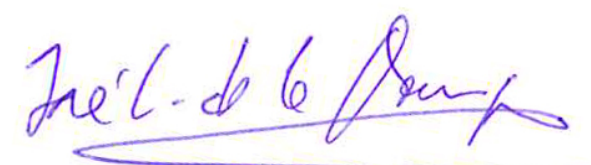

Dr. José Luis de la Pompa Mínguez

Thesis Director 

This doctoral thesis was performed in the Intercellular Signaling in Cardiovascular Development and Disease laboratory directed by Dr. José Luis de la Pompa at Centro Nacional de Investigaciones Cardiovasculares (CNIC) in Madrid.

This study was financed by different grants of Ministerio de Ciencia, Innovación y Universidades (SAF2013-45543-R, SAF2015-71863-REDT y SAF2016-78370-R), CIBER Cardiovascular. Instituto de Salud Carlos III. Ref.: CB16/11/00399, Red de Terapia Celular. Instituto de Salud Carlos III. Ref.: RD16/0011/0021, CardioNeT- International Training Network on the Cellular and Molecular Bases of Heart Homeostasis and Repair. Agency: European Union, ref. 28600, 2012-2015.

Dimitrios Grivas holds a PhD fellowship linked to the grant [PITN-GA-2011-289600] (CardioNeT) 

To my father

Panagiotis Grivas 

Acknowledgments 
All these years I met so many people that one way or another contributed to the completion of this work. I would like to start with Jose Luis and I would like to thank him for the opportunity to work in his laboratory, for his support and help all these years. Many thanks to the people that help me with the aquarium and the animals. So, I would like to thank Bea for all her kindness, support and her help with the genotyping and the organization of all the line that we had. Eduardo for his willingness to provide anything I needed and his help. Also, Vanesa E. for her help with the fish and the genotyping although her short pass from the lab, as well as Lucia.

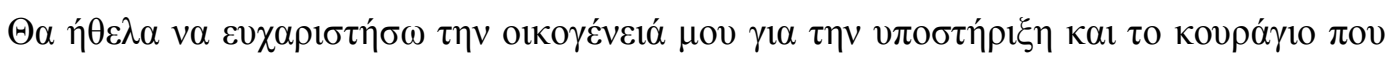

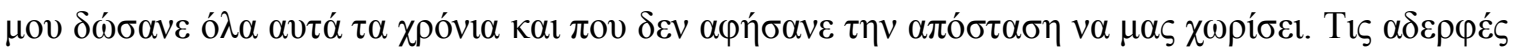

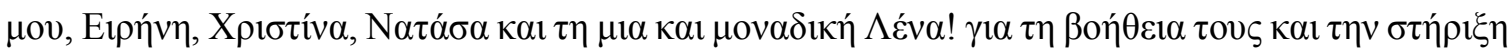

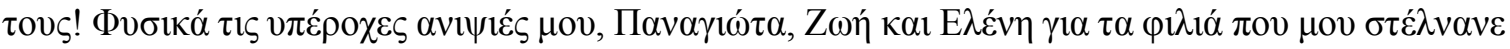

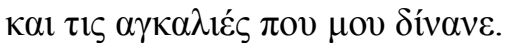

A big thank to all the members of the lab. Marcos for always been available, Luis for his help, Donal, Belen, Quim, Vanesa B., Rebeca T., Rebeca P., Vera, Alexandro, Tamara and Christina for all the fun moments and their help. I could not forget former members of the lab, $\Sigma^{\prime}$

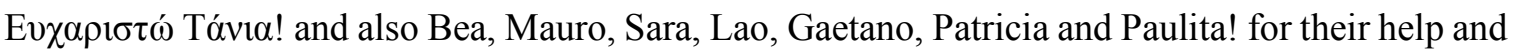
the fun. Vitor for his help, support and all the nice discussion we had. And of course, my former zebra-mates, Juliane for her support and help, and Alvaro for introducing me to these projects.

I would like to thank Veronica from the microscopy for all her patience with my requests and her significant help with the macros we designed, and the imaging. Fernando form the bioinformatics for his help with the modelling and for all the nice discussion, and Roisin for her beautiful sections. Carlos and Ricardo from the ICMM for the fruitful collaboration and their help. Dr. Peter F. Hitchcock from the University of Michigan, USA for sharing with us the Mdka antibody.

I would like also to thank Nadia for her help and all the members of her laboratory Laura, Hector, Marina, Anders, Marco and Juan Manuel for sharing reagents, especially Maria for all her help and support. As well as the 'beneditos', the 'torres', the 'flores' and the 'martins' for their help, Moreno for all the interesting conversations we had, and of course Wen, Jose and Isaak for all the fun during the weekends.

Many thanks to the Cardinet mates, Ghislaine, Alberto, Mayyasa, Megan M., Andrea, Megan R, Francesco, Nils, Adriana, Vikas, Chrysanthi, Enrique, Stuti, Noémie for all the nice conversations, the fun and the good times we had during our travels.

It's been one hell of a ride, gracias chiquititos! And as always, BE fantastico fenomenal maraviglioso!!! 
Summary 
Zebrafish have the ability to regenerate cardiac tissue after injury in contrast to mammals. Cryoinjury of the heart triggers a regenerative program that includes inflammatory response, cell proliferation and formation of a transient scar that eventually lead to complete tissue regeneration. This thesis focused on understanding the molecular and mechanical properties governing zebrafish heart regeneration. In particular, we studied the role of midkine-a ( $m d k a)$ and caveolin-1 (cavl), two genes that were upregulated in a microarray analysis of regenerating hearts performed in the laboratory. Additionally, we studied the epigenetic regulation of $m d k a$ and tested in vivo different $m d k a$ cis-regulatory elements with the goal to identify the minimum regulatory regions that drive $m d k a$ expression in development and after injury.

Midkine-a (Mdka) is a neurite-growth factor that is involved in the formation of the media floor plate in zebrafish. Here, we show that $m d k a$ expression was strongly induced after heart injury, although it was not expressed in intact hearts. In injured hearts, the onset of $m d k a$ expression was one-day post cryoinjury (dpci) in all the epicardial layer, whereas by $7 \mathrm{dpci}$ the expression became restricted to the epicardial cells covering the injured area. To study the role of Mdka in heart regeneration, we generated $m d k a$-knock out (KO) zebrafish strains. In injured heart, $m d k a$ depletion did not trigger upregulation of $m d k b$ and $p t n$, the two other members of the midkine gene family, which might compensate for the loss of Mdka. Analysis of 90dpci hearts showed that $m d k a$ deletion resulted in impaired heart regeneration, with the injured area enriched in collagen deposition. However, the proliferation ratio of cardiomyocytes (CM) and epicardial cells was not affected. Analysis of extracellular matrix turnover, immune cells infiltration in the injured area and revascularization of the regenerating tissue will clarify the reasons for the impaired cardiac regeneration in $m d k a-K O$ hearts.

During development, $m d k a$ is expressed in neural tissues such us neural tube and brain, and is not detected neither in developing hearts nor in adult intact hearts. However, $m d k a$ expression was activated upon heart injury, having dynamic expression pattern. To investigate such a change in the expression pattern, we used available ChIP-seq and ATAC-seq data to analyse the epigenetic landscape of $m d k a$. In $m d k a$ locus, there are three intronic enhancer sequences and two additional enhancers located upstream of $m d k a$. We tested these enhancers and the $m d k a$ promoter linked to GFP reporter and studied their expression patterns in our transgenic lines. We found that the promoter of $m d k a$ and the intronic enhancers were responsible for the spatio-temporal expression pattern of $m d k a$ during development, in adult tissues and after injury of the heart and the fin.

Caveolin-1 (Cav1) is the structural protein of caveolae, small membrane invaginations that are involved in signal transduction and mechanoprotection. Our expression analysis showed that Cav1 was expressed in the endocardium, endothelium and epicardium of intact adult heart. After heart cryoinjury, Cav1 was strongly expressed in epicardial cells covering the injury, and in the endocardium invading the damaged tissue. We introduced mutations in cavl sequence that resulted in loss of Cav1 and caveolae. Analysis of the regeneration process revealed that the caveolae- 
depleted hearts regenerated normally, although cavl-KO hearts showed a transient defect on CM proliferation. However, loss of Cav1 and caveolae affected heart function, since the ejection fraction and the heart rate were significantly decreased in intact hearts. Using atomic force microscopy, we found that epicardial cells and cortical cardiomyocytes lacking caveolae were stiffer than the control hearts explaining the decline of cardiac function. 
El pez cebra, al contrario de los mamíferos, tiene la capacidad de regenerar el tejido cardíaco tras sufrir un daño. La criolesión cardíaca desencadena un programa de regeneración tisular que incluye una respuesta inflamatoria, la inducción de proliferación celular y la formación de una cicatriz transitoria que lleva a la regeneración completa del tejido dañado. El objetivo de esta tesis se centra en el estudio de las propiedades moleculares y regenerativas del corazón del pez cebra. En concreto, se ha estudiado el papel de los genes midkina-a ( $m d k a)$ y caveolina-1 (cavl), ambos identificados en corazones en regeneración, a partir de un análisis de microarray de ADN llevado a cabo en nuestro laboratorio. Además hemos estudiado la regulación epigenética de mdka y para ello hemos testado elementos reguladores en cis de mdka con el objetivo de identificar las secuencias regulatorias mínimas responsables de dirigir la expresión de mdka durante el desarrollo y tras daño.

Midkine-a (Mdka) es un factor de crecimiento de neuritas, implicado en la formación de la placa basal medial en el pez cebra. En este trabajo mostramos el fuerte aumento de expresión de $m d k a$ inducido por daño cardíaco, a pesar de que no se expresa en corazones sanos. En corazones dañados, el pico de expresión de $m d k a$ se observa un día después de la criolesión (abreviado en inglés como dpci) en toda la capa del epicardio, mientras que, a los 7dpci, su expresión se restringe a las células de epicardio que envuelven el área dañada. Para estudiar la función de Mdka en la regeneración del corazón, generamos líneas mutantes deficientes en $m d k a$ (abreviado como $m d k a$ $K O$, siglas del término en inglés knock-out). En corazones dañados, la eliminación de $m d k a$ no indujo la sobreexpresión de $m d k b$ o $p t n$, los otros dos miembros de la familia génica midkine, como mecanismo de compensación a la pérdida de $m d k a$. El análisis de corazones a los 90dpci desveló que la deleción de $m d k a$ condujo a un defecto en la regeneración cardíaca y a un aumento en la deposición de colágeno en el área dañada. Sin embargo, la proliferación de los cardiomiocitos (CM) y de las células del epicardio no se vio afectada. Un análisis más profundo del recambio de la matriz extracelular, la infiltración de células inmunes en el área lesionada y la revascularización del tejido en regeneración, aclararán las causas de la regeneración cardiaca deficiente en los corazones $m d k a-$ $K O$.

Durante el desarrollo, mdka se expresa en tejidos neurales, como el tubo neural y el cerebro, pero no se detecta ni en el corazón en desarrollo ni en el corazón adulto. Sin embargo, la expresión de mdka se induce tras daño cardíaco, mostrando un patrón de expresión dinámico. Para investigar este cambio en el patrón de expresión, hemos utilizados los datos disponibles procedentes de análisis de ChIP-seq y ATAC-seq para analizar el escenario epigenético de mdka. En el locus de mdka, existen tres secuencias intrónicas y dos secuencias localizadas upstream de mdka, de actividad enhancer. Testamos estas secuencias unidas a un gen reportero GFP y/o al promotor de Mdka y estudiamos el patrón de expresión de GFP en nuestras líneas transgénicas. Encontramos las secuencias enhancer responsables de la expresión espacio-temporal de mdka durante el desarrollo, en tejido adulto y tras daños en el corazón y en la aleta. 
La Caveolina-1 (Cav1) es una proteína estructural de las caveolas, pequeñas invaginaciones de la membrana plasmática celular, involucradas en la transducción de señales y la mecanoprotección. Nuestro análisis de expresión mostró que Cav1 se expresa en el endocardio, endotelio y epicardio del corazón adulto. Después de la inducción de daño cardíaco por criolesión, se detectó una alta expresión de Cav1 a los 7 dpci, en las células epicárdicas que envolvían el área de la lesión, así como en las células endocárdicas que invadían el tejido lesionado. Generamos mutaciones en la secuencia de Cav1 que condujeron a la pérdida de Cav1 y de las caveolas. El análisis del proceso de regeneración desveló que los corazones deficientes en Cav1 se regeneraron de forma correcta, aunque detectamos un defecto transitorio en la proliferación de los $\mathrm{CM}$ en los cav1-KO corazones. Sin embargo, la pérdida de Cav1 y de caveolas afectó a la función cardiaca ya que confirmamos una disminución significativa de la fracción de eyección y de la frecuencia cardíaca en los cav1-KO corazones sanos. Mediante el uso de microscopía de fuerza atómica, encontramos que las células de epicardio y los $\mathrm{CM}$ corticales que carecían de caveolas, presentaban una mayor rigidez que en los corazones control, lo que explicaba la disminución de la función cardíaca en estos cavl-KO. 
Table of Contents - Indexes 


\section{Table of Contents}

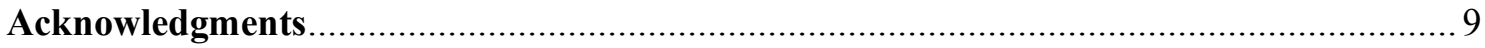

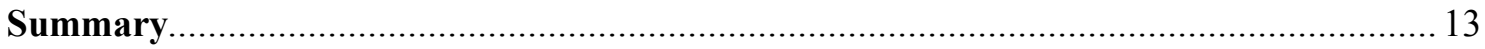

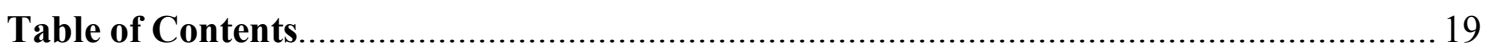

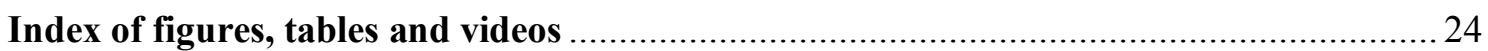

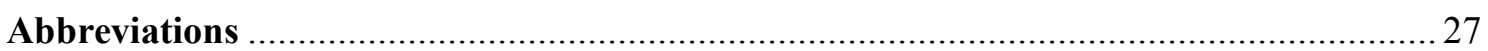

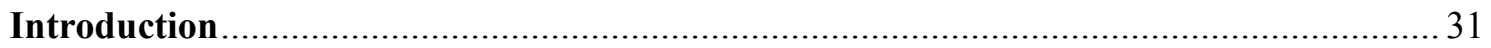

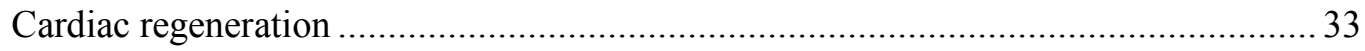

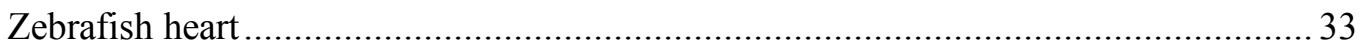

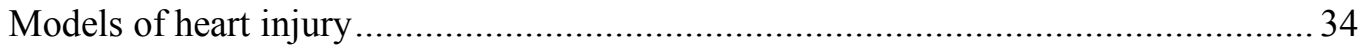

Zebrafish heart regeneration ................................................................................. 35

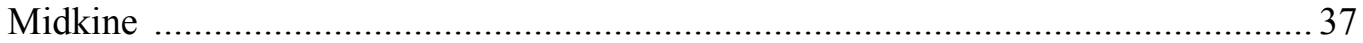

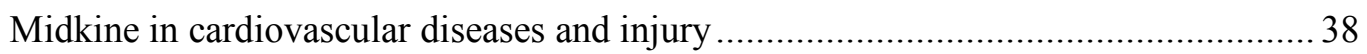

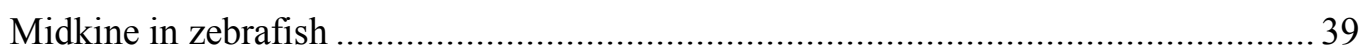

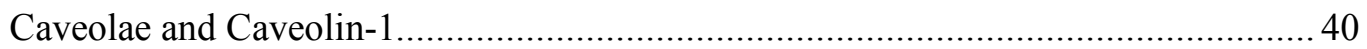

Caveolins expression, structure and caveolae assembly ........................................... 40

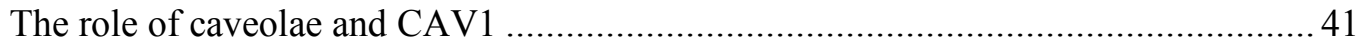

The role of CAV1 in the mammalian heart ............................................................... 43

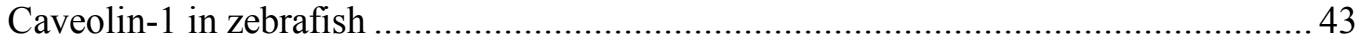

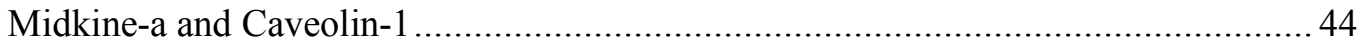

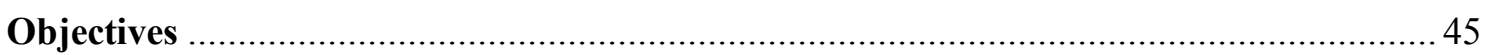

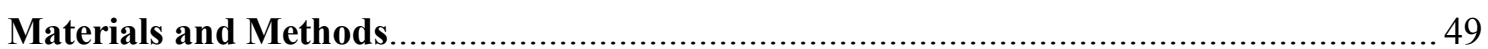

Zebrafish husbandry and transgenic lines ............................................................... 51

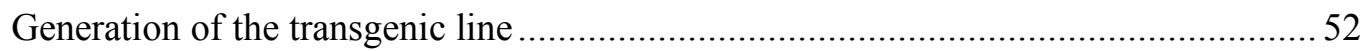

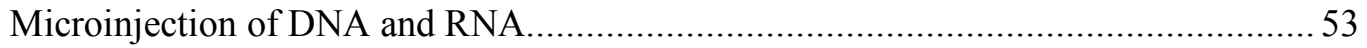

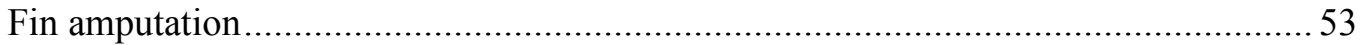

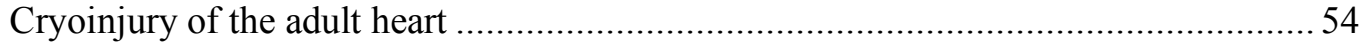

Bromodeoxyuridine (5-bromo-2'-deoxyuridine - BrdU) injection ............................... 54

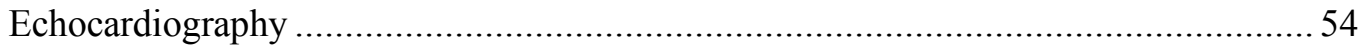

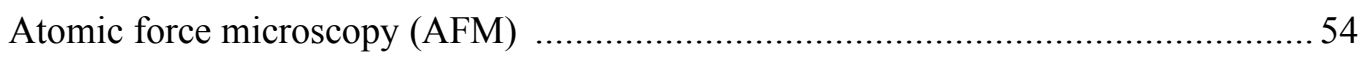

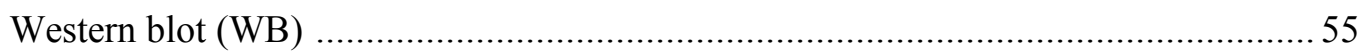

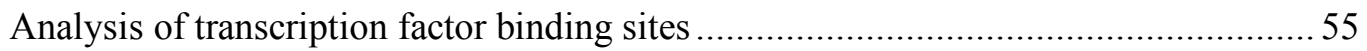

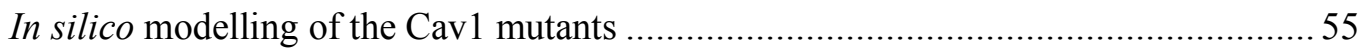

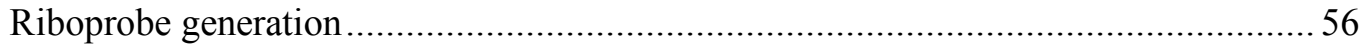

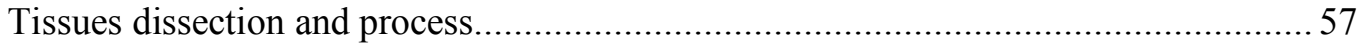

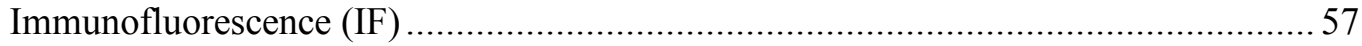




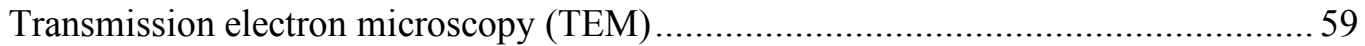

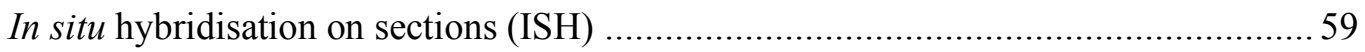

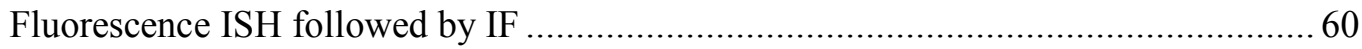

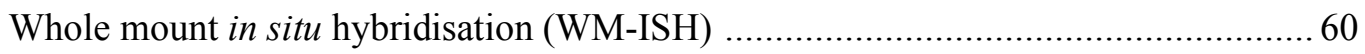

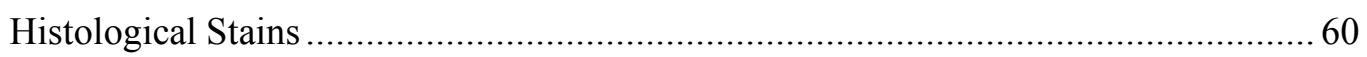

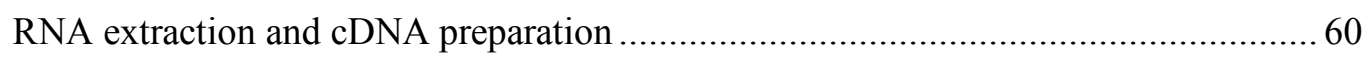

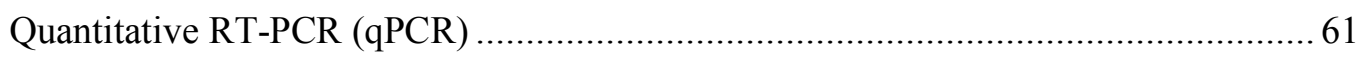

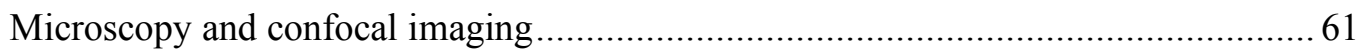

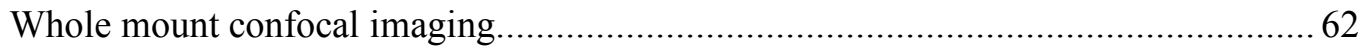

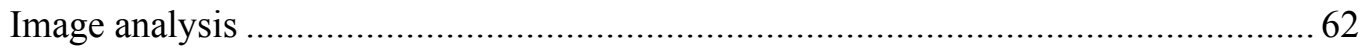

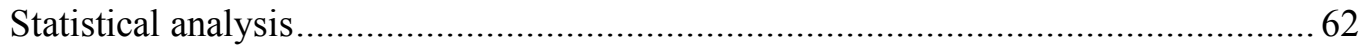

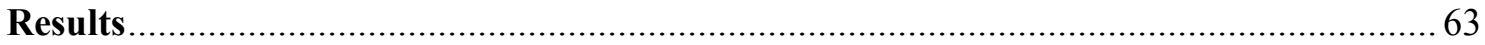

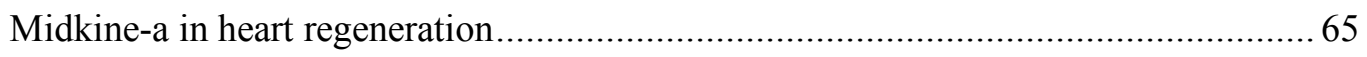

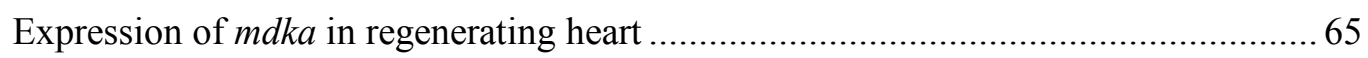

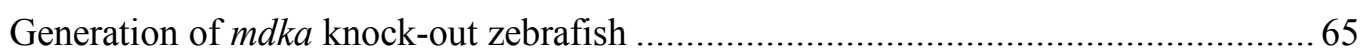

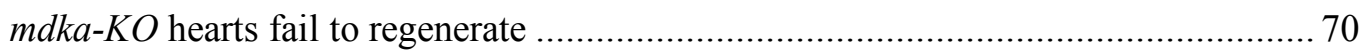

Cell proliferation analysis in $m d k a^{-/-}$regenerating hearts ............................................ 71

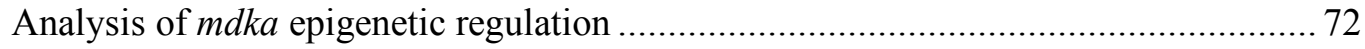

The epigenetic landscape of $m d k a$ locus in development and heart regeneration ......... 72

Analysis of $T g(m d k a: G F P)$ and $T g(e n h: G F P)$ transgenic lines ............................... 74

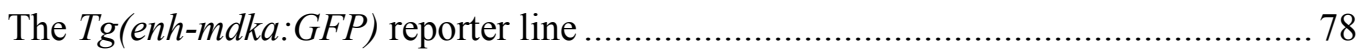

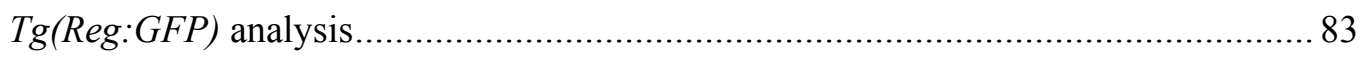

Enhancer-4 and enhancer-5 in development and regeneration ................................. 88

Transcription factors analysis on $m d k a$ cis-regulatory elements ............................... 91

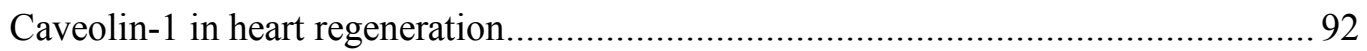

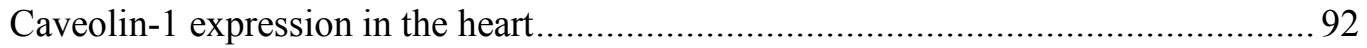

Caveolin-2 and Cavin1 expression in control and injured adult hearts........................ 94

Generation of cavl-KO zebrafish by CRISPR/Cas9 .................................................. 94

Analysis of Cav1a and Cav1b expression in X136 and X166 hearts........................... 98

Cavin1b expression in the X136 and X166 hearts.................................................. 101

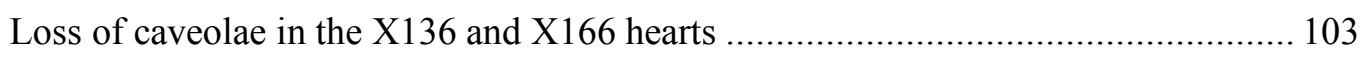

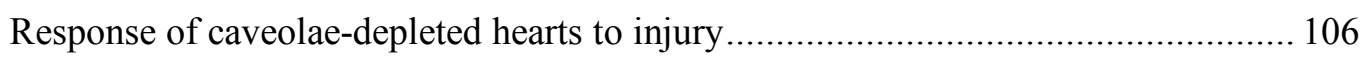

TGF $\beta$ signalling activation is unaffected in X136 hearts .......................................... 108

Epicardial and endocardial cell function in X136 heart is unaffected ........................ 109

Cardiomyocytes proliferation is transiently affected in injured X136 hearts ............. 111

Impaired heart function in the X136 animals .......................................................... 112

Depletion of caveolae affects cardiac elasticity .................................................... 112 


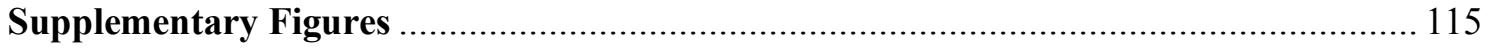

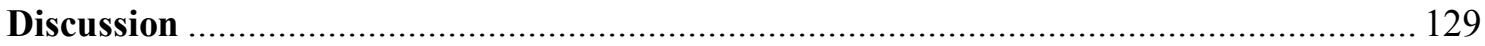

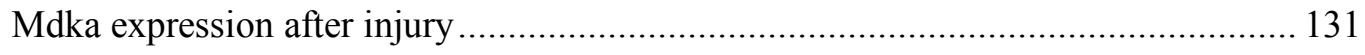

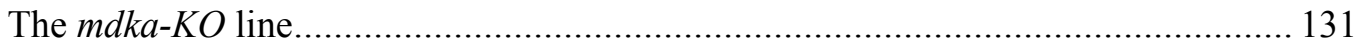

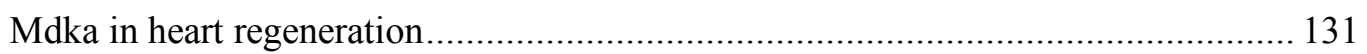

Dissection and in vivo evaluation of $m d k a$ epigenetic landscape ............................ 134

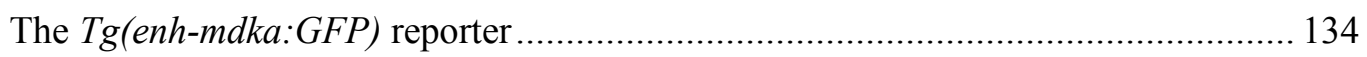

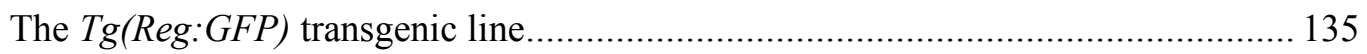

The $\operatorname{Tg}($ enha4:GFP) and $\operatorname{Tg}($ enha5:GFP) transgenic lines ..................................... 135

Conclusions of cis-regulatory elements study ….................................................. 136

Cav1 expression in intact and injured heart........................................................... 137

Generation of the cavl-KO zebrafish.................................................................... 137

Cav1 is not required for adult zebrafish heart regeneration after cryoinjury............... 138

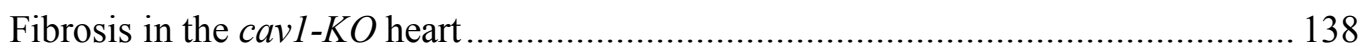

Cav1 is transiently required for CM proliferation during regeneration...................... 139

Cav1 is necessary for proper heart function and cardiac cell stiffness....................... 140

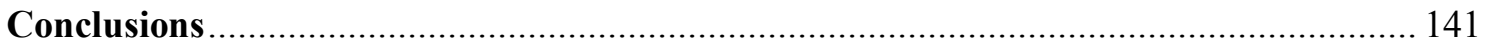

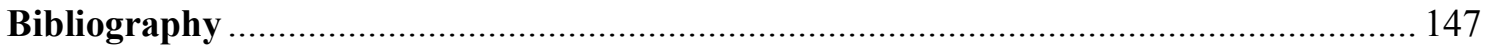




\section{Index of figures, tables and videos}

\section{Figure index}

Introduction

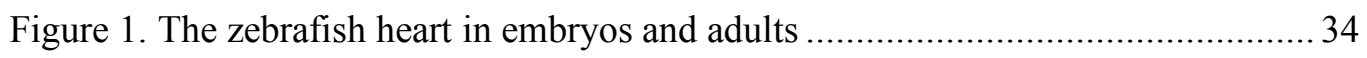

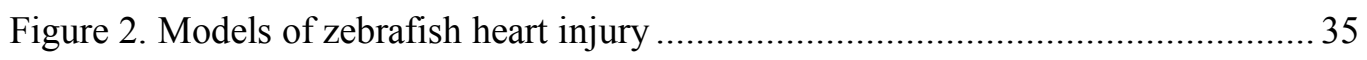

Figure 3. Caveolae and Caveolin-1 domain organization ......................................... 42

\section{Results}

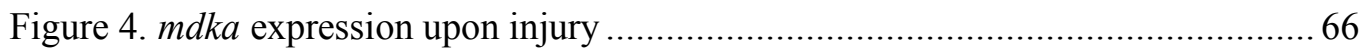

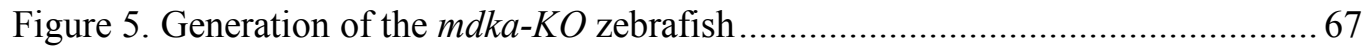

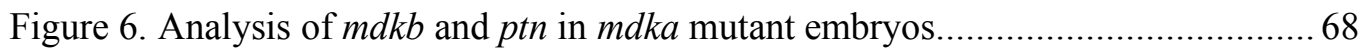

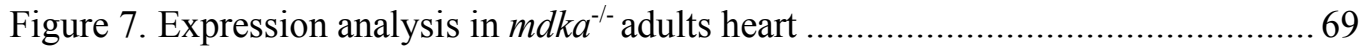

Figure 8. Loss of Mdka leads to arrest of heart regeneration ..................................... 70

Figure 9. Epicardial cell and CM proliferation in $m d k a^{-/}$hearts ................................ 71

Figure 10. $m d k a$ locus and epigenetic marks in development and heart regeneration ... 73

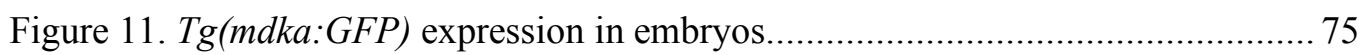

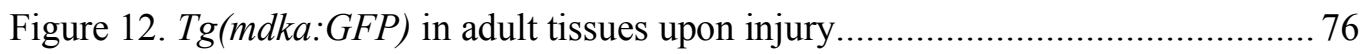

Figure 13. $\operatorname{~gg(enh:GFP)~expression~in~embryos~and~adults~........................................~} 77$

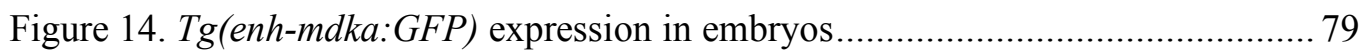

Figure 15. Expression analysis of $T g(e n h-m d k a: G F P)$ embryos by FISH/IF .............. 80

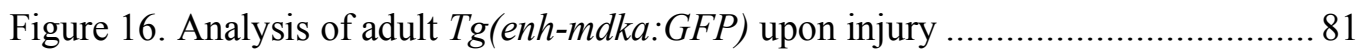

Figure 17. Examination of the GFP expression in $\operatorname{Tg}($ enh-mdka:GFP) adult neural

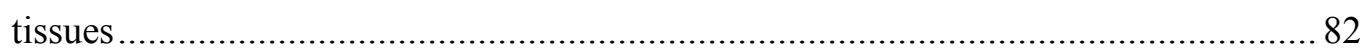

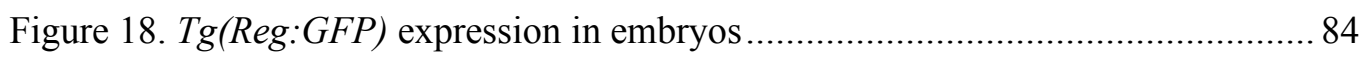

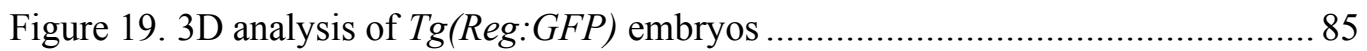

Figure 20. Immunofluorescence analysis of $\operatorname{Tg}(\operatorname{Reg}: G F P)$ embryos............................ 86

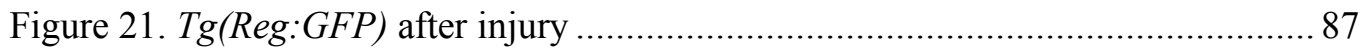

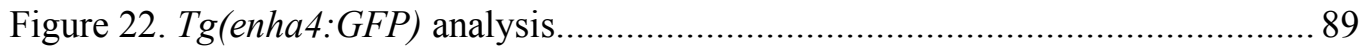

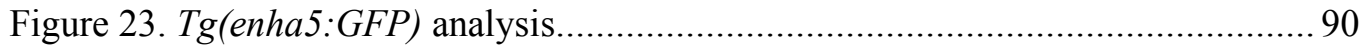

Figure 24. Expression analysis of Cav1 in the adult heart ........................................... 93

Figure 25. Caveolin-2 and Cavin1 expression in control and regenerating heart .......... 95

Figure 26. Generation of cav1-KO by CRISPR/Cas9 and transcriptional analysis ....... 97

Figure 27. Expression analysis of Cav1a in X136 and X166 hearts ............................ 99

Figure 28. Expression analysis of Cav1b in X136 and X166 hearts......................... 100

Figure 29. WB analysis of Cav1 in X136 and X166 hearts....................................... 101

Figure 30. Cavin1b expression in the X136 and X166 hearts ................................. 102

Figure 31. Caveolae abundance in adult heart ......................................................... 104 
Figure 32. Loss of caveolae in X136 and X166 hearts ........................................... 105

Figure 33. Monitoring of the regeneration process in X136 hearts ............................ 107

Figure 34. Investigation of X166 hearts regeneration............................................. 108

Figure 35. TGF $\beta$ signalling activation in injured caveolae-less heart........................ 109

Figure 36. Epicardial proliferation and endocardial behaviour in X136 hearts after

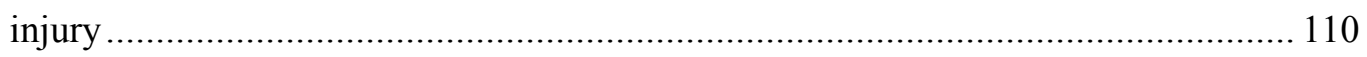

Figure 37. CM proliferation upon injury in X136 hearts ......................................... 111

Figure 38. Echocardiography analysis of WT and X136 hearts .............................. 112

Figure 39. Loss of caveolae leads to stiffer cardiac tissue ….................................... 113

\section{Supplementary Figure}

Supplementary Figure 1. Expression of $m d k a$ in control and regenerating hearts....... 117

Supplementary Figure 2. Epigenetic marks in $m d k a$ locus 48hpf............................. 118

Supplementary Figure 3. $\mathrm{Tg}($ enh-mdka:GFP) expression at $15 \mathrm{hpf} . . \ldots \ldots \ldots \ldots \ldots \ldots \ldots \ldots . . . . . . . . . . . .118$

Supplementary Figure 4. $\operatorname{Tg}($ enh-mdka:GFP) animals show GFP expression only in regenerating fin

Supplementary Figure 5. Immunofluorescence of GFP and zn3 or zn12 in $\operatorname{Tg}(\operatorname{Reg}: G F P)$ embryos

Supplementary Figure 6. Cav1 expression in embryonic zebrafish and mouse heart.. 121

Supplementary Figure 7. In silico 3D modelling and analysis of the Cav1 mutant proteins

Supplementary Figure 8. Expression analysis of Cav1 in X135 and 115del hearts.... 124 Supplementary Figure 9. cav1a deletion by CRISPR/Cas9 and Cav1 expression analysis in cavla-KO hearts 125

Supplementary Figure 10. WB analysis of Cavin1 in X136 hearts 126

Supplementary Figure 11. Analysis of collagen and fibrin of the injured area and ventricular volume 126

Supplementary Figure 12. Analysis of interstitial fibrosis in WT and X136 hearts ... 127

Supplementary Figure 13. X166 CM proliferation upon injury 127

\section{Table index}

Table 1. Zebrafish strains used in this thesis 51

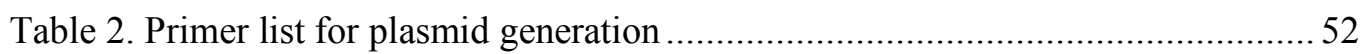

Table 3. Primers for genotyping the CRISPR/Cas9 lines............................................... 53

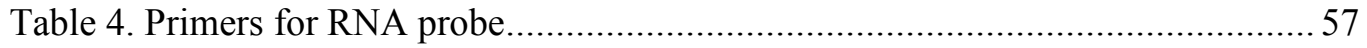

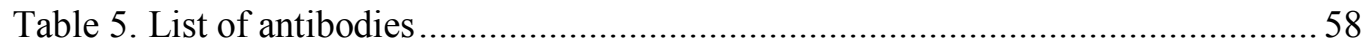

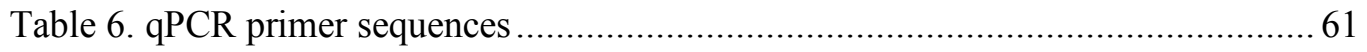


Table 7. TF binding sites in $m d k a$ cis-regulatory elements 91

\section{Video index}

Video $1 \mathrm{Tg}$ (enh-mdka:GFP) 5dpf brain

Video $2 \mathrm{Tg}$ (enh-mdka:GFP) cranial neuromast

Video $3 \operatorname{Tg}$ (Reg:GFP) 1 dpf endogenous GFP in the brain

Video $4 \mathrm{Tg}$ (Reg:GFP) 36hpf brain dorsa-ventral view

Video $5 \mathrm{Tg}(\operatorname{Reg}: G F P)$ 36hpf brain lateral view

Video $6 \operatorname{Tg}(\operatorname{Reg}: G F P) / \operatorname{Tg}(f l i l a: D s e d e x) 2 \mathrm{dpf}$ trunk

Video $7 \operatorname{Tg}(\operatorname{Reg}: G F P) / \operatorname{Tg}(f l i 1 a: D s e d e x) 2$ dpf trunk axons

Video $8 \mathrm{Tg}$ (Reg:GFP) $6 \mathrm{dpf}$ trunk lateral line with muscle

Video $9 \operatorname{Tg}($ Reg:GFP) 6dpf trunk neural tube

Video $10 \mathrm{Tg}$ (Reg:GFP) 6dpf cranial neuromast zns5

Video 11 X136 WT Tg(flila:GFP)/Tg(myl7:mRFP) 7dpci

Video 12 X136 KO Tg(flila:GFP)/Tg(myl7:mRFP) 7dpci 
Abbreviations 
AFM

AFOG

Aldh1a2

ATAC

$\mathrm{Bcl} 2$

BrdU

cg1

cg2

ChIP

Creb1

CRISPR

CSD

CUBIC

dpa

dpci

dpf

dpi

ECM

EF

enh

enha4

enha5

FISH

gfap

hpci

hpf

HUVEC

ICAM

ins

IP

ISH

LAD

Mdk

Mdka

Mdkb

mg1

MI
Atomic force microscopy

Acid Fuchsin Orange-G

Aldehyde Dehydrogenase 1 Family, Member A2

Assay for transposase-accessible chromatin

B-cell lymphoma 2

Bromodeoxyuridine

Caveolin guide 1

Caveolin guide 2

Chromatin immunoprecipitation

CAMP responsive element binding protein 1

Clustered regularly interspaced short palindromic repeats

Caveolin scaffolding domain

Clear, unobstructed brain imaging cocktails

Days post amputation

Days post cryoinjury

Days post fertilization

Days post injury (ventricular resection)

Extracellular matrix

Ejection fraction

enhancer

enhancer4

enhancer5

Fluorescence ISH

Glial fibrillary acidic protein

Hours post cryoinjury

Hours post fertilization

Human umbilical vein endothelial cell

Intercellular adhesion molecule

Insulator

Intraperitoneal injection

In situ hybridization

Left anterior descending artery

Midkine

Midkine-a

Midkine-b

mdka guide 1

Myocardial infarction 


\begin{tabular}{ll} 
mmp & Matrix metalloproteinase \\
mRFP & Membrane bound red fluorescent protein \\
myl7 & Myosin Light Chain 7 \\
psmad3 & Phospho-smad3 \\
Ptn & Pleiotrophin \\
qPCR & Quantitative real-time PCR \\
RA & Retinoic acid \\
RhoA & Ras homolog family member A \\
RNA-seq & RNA sequencing \\
Rxra & Retinoic acid receptor RXR-alpha \\
SMC & Smooth muscle cells \\
Spi1 & Transcription factor Pu.1 \\
TAC & Transverse aortic constriction \\
TEM & Transmission electron microscopy \\
TGF $\beta$ & Transforming growth factor beta \\
TGFßR1 & Transforming growth factor beta receptor 1 \\
UCSC & University of California, Santa Cruz \\
WB & Western blot \\
WM-ISH & Whole-mount in situ hybridization \\
X.Card.Actin & Xenopus cardiac actin \\
ZED & Zebrafish enhancer detection vector \\
\hline
\end{tabular}


Introduction 


\section{Cardiac regeneration}

The mammalian heart has limited regeneration capacity. Myocardial infarction (MI) results in massive cell death of cardiomyocytes (CM) that are not replaced. This leads to formation of a permanent fibrotic scar to protect the integrity of the myocardial wall (Souders et al., 2009). The scar tissue is not resolved and without contractile abilities the heart increases cardiac output to maintain function (St. John Sutton and Sharpe, 2000). Progressively, pathological cardiac remodelling occurs, including ventricular hypertrophy and dilatation that eventually lead to heart failure and increased mortality (Benjamin et al., 2019). Neonatal murine hearts have a small regeneration capacity, where damaged myocardium is replaced by CM proliferation, that is lost in adults (Porrello et al., 2011; Zogbi et al., 2014). Conversely, some lower vertebrates such as newt (Bader and Oberpriller, 1978), axolotl (Vargas-González et al., 2005) and zebrafish (Poss et al., 2002) have the remarkable ability to regenerated cardiac tissue after injury. In particular, the regeneration potential of zebrafish extends to many organs, such as brain (Kroehne et al., 2011), retina (Vihtelic and Hyde, 2000), spinal cord (Becker et al., 1997) and fin (Johnson and Weston, 1995), making it a very suitable model for regeneration studies.

\section{Zebrafish heart}

The zebrafish heart develops from a linear heart tube after fusion of mesodermal progenitor cells ten-hours post fertilization (hpf) (Yelon et al., 1999). By 24hpf, contraction and circulation begin, followed by looping of the cardiac tube to form two chambers, the ventricle and the atrium (Reischauer et al., 2014). Approximately three-days post fertilization (dpf), ventricular CM delaminate and form trabeculae that remain in adults since myocardium does not undergo compaction (Figure 1A) (Cherian et al., 2016). In parallel, proepicardial cells begins to cover the heart (Peralta et al., 2013) and the atrioventricular and aortic valves are formed from endocardial cushions (Pestel et al., 2016). The cortical myocardial wall is formed later (six-weeks post fertilization) by trabecular CM migration (Gupta and Poss, 2012) and around three-months post fertilization, coronary vasculature is established from sprouting and migration of endocardialderived endothelial cell (Harrison et al., 2015). The adult zebrafish heart is non-septated and is characterized by a 'spongy' trabecular myocardium (Figure 1B, C) (Sedmera et al., 2000). Between the cortical (outer) and the trabecular (inner) myocardium there is a junctional zone that contains fibroblasts and the primordial CM (Lafontant et al., 2013; Pfefferli and Jaźwińska, 2017). The epicardium covers the cortical layer that abounds of coronary veins while the endocardium separates the trabecular layer from the lumen. The ventricle is separated from the atrium with the atrioventricular valve and from the bulbus arteriosus (outflow track) with the bulboventricular valve (Hu et al., 2001). 

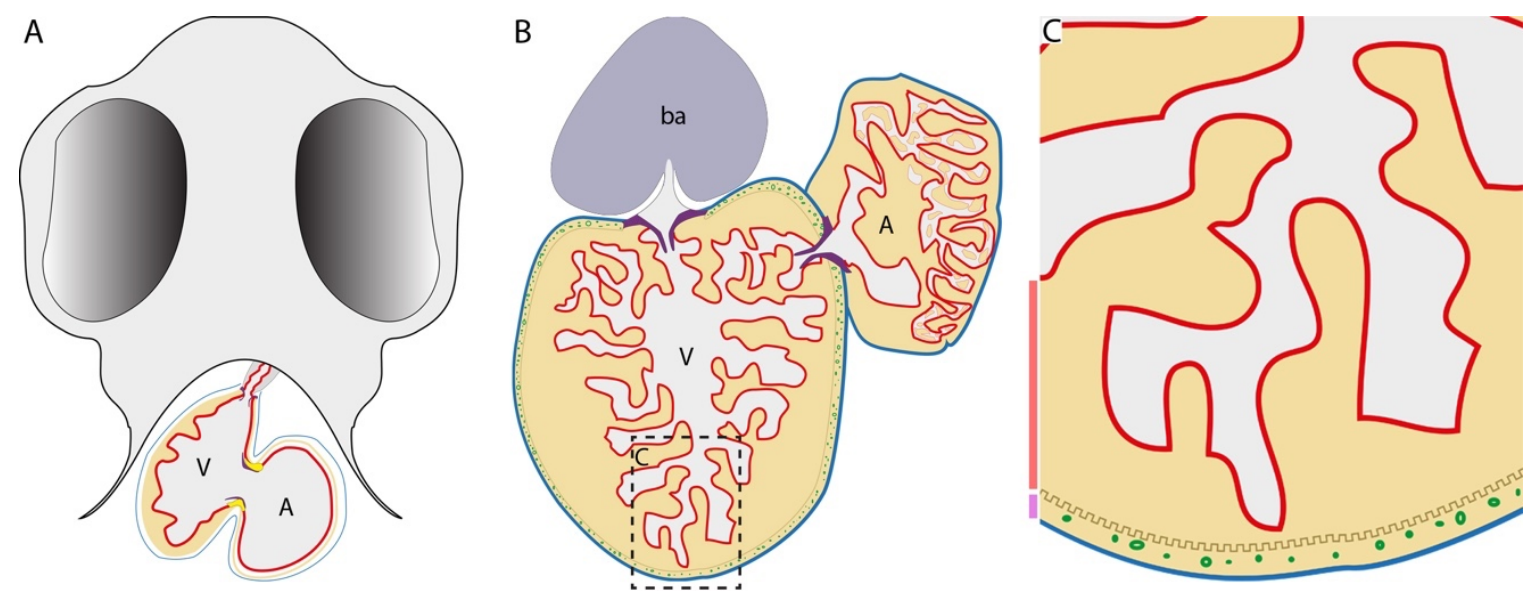

Endocardium Myocardium

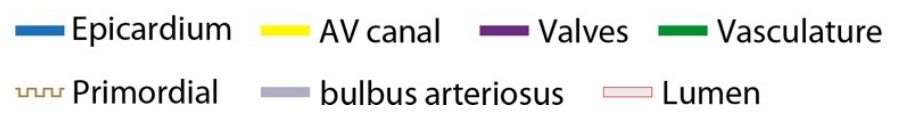

Figure 1. The zebrafish heart in embryos and adults

(A) Ventral view illustration of developing heart 3-5dpf. The three main cardiac cell layers endocardium, myocardium and epicardium are colour-indicated. (B, C) Drawing of adult heart with the 'spongy' trabecular, the flatten primordial and the outer cortical myocardium as well as the endo-, epicardium and the coronary vasculature. (C) Magnification of the ventricular apex in B. V $=$ ventricle, $\mathrm{A}=$ atrium, $\mathrm{ba}=$ bulbus arteriosus, $\mathrm{AV}=$ atrioventricular.

\section{Models of heart injury}

Different types of injuries have been used to study heart regeneration, including ventricular cryoinjury (cryocauterization), ventricular resection and genetic ablation of CM (Figure 2). Cryoinjury of the ventricle with a copper probe embedded in liquid nitrogen results in significant cell death and formation of a transient scar tissue, that regenerates within 90-days (Figure 2A) (Chablais et al., 2011; Gonzalez-Rosa et al., 2011; Schnabel et al., 2011). Ventricular resection was the first model used, in which the cardiac apex is surgically removed, followed by the formation of a fibrin clot and replacement of the lost tissue in 60-days (Figure 2B) (Poss et al., 2002). Genetic cell ablation is another approach to induce injury. In this model, cells type-specific (CM or epicardial cells) are depleted by genetic induction of cytotoxic agents (Figure 2C) (Wang et al., 2011; Wang et al., 2015).

In all these models the heart fully regenerates the damaged tissue and recovers functionally. Additionally, inflammatory response as well as epicardium, endocardium and CM activation occur independently of the method (Chablais et al., 2011; Gonzalez-Rosa et al., 2011; Poss et al., 2002; Schnabel et al., 2011; Wang et al., 2011). However, there are some significant differences. Cryoinjury results in tissue death and apoptosis in all cell types around the injured site, whereas in the resection model, apoptosis is limited. However, the most important difference is the formation of the fibrotic scar. In cryoinjury, the scar tissue is substantial, resembling the human MI infarction (Chablais et al., 2011; Gonzalez-Rosa et al., 2011; Schnabel et al., 2011). In contrast, fibrosis is 
minimum in the resection model (Poss et al., 2002). Additionally, evidence suggests that the regeneration response between cryoinjury and resection differs, since the cardiac lymphatics are activated upon cryoinjury, but not after resection (Vivien et al., 2019).
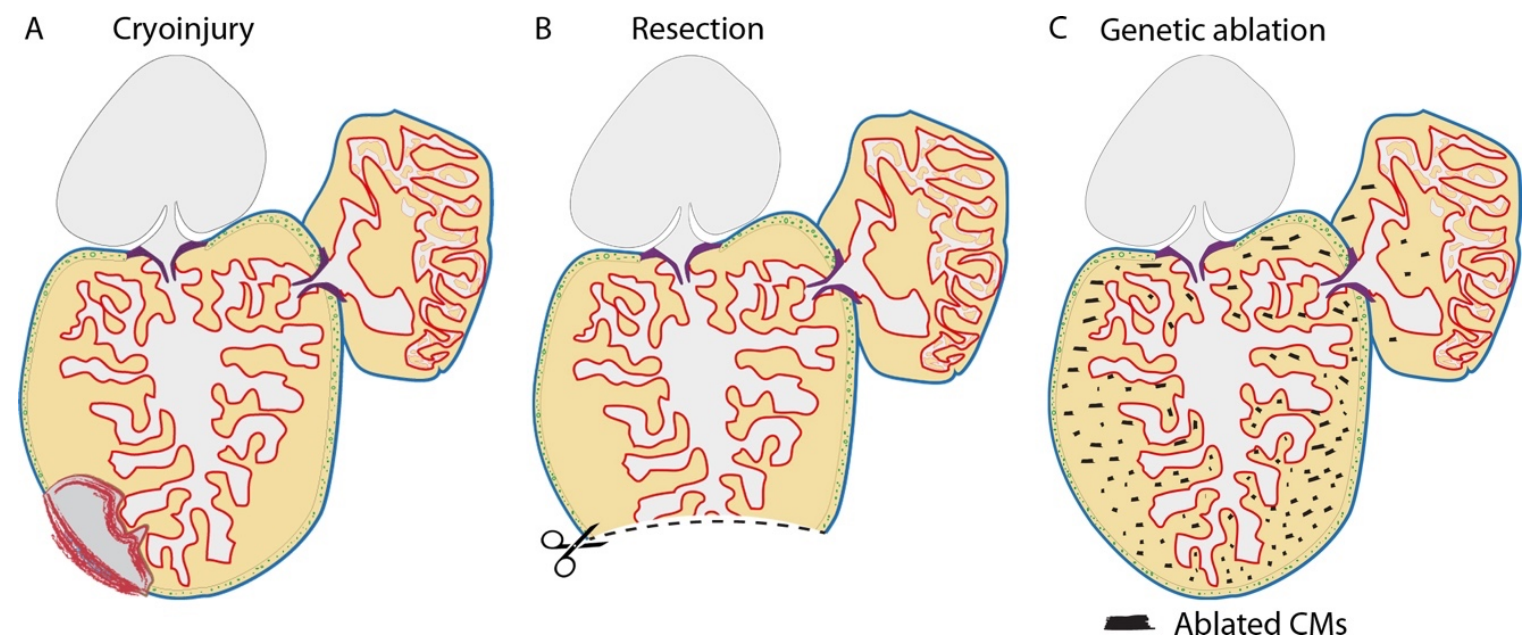

\section{Figure 2. Models of zebrafish heart injury}

(A) Cryoinjury leads to severe cell death and to a fibrotic scar. (C) Removal of the ventricular apex by amputation is the resection approach. (C) Genetic ablation of CM results in scattered CM death in the chambers.

\section{Zebrafish heart regeneration}

Injury of the zebrafish heart triggers a sequence of events including inflammatory response, epicardium and endocardium activation as well as CM proliferation, and fine tuning of these processes is necessary for proper regeneration.

Inflammation is detected hours after the injury and expression of cytokines props further immune cells recruitment (Huang et al., 2013). Inhibition of inflammation leads to decreased cell proliferation, impaired angiogenesis and thus incomplete regeneration (De Preux Charles et al., 2016; Huang et al., 2013). Additionally, delayed immune response is associated with impaired vascularization and resolution of the scar tissue (Lai et al., 2017), whereas long retention of neutrophils in the wound promotes revascularization (Xu et al., 2019).

The epicardium is activated early after injury, proliferates and moves towards the damaged area (Gonzalez-Rosa et al., 2011; Lepilina et al., 2006). $W t 1 b^{+}$(an epicardial marker gene activated upon injury) and $t c f 21^{+}$(a transcription factor gene and epicardial cell marker in zebrafish) epicardial cells give rise to perivascular cells and myofibroblasts (González-Rosa et al., 2012; Kikuchi et al., 2011a). Migration of the epicardium depends on Hedgehog signalling and depletions of epicardial cells reduces CM proliferation and arrests heart regeneration (Wang et al., 2015). Furthermore, Fgf and Pdgf signalling induce epithelial to mesenchymal transition (EMT) and 
blockage of these pathways leads to reduced vascularization and impaired regeneration (Kim et al., 2010; Lepilina et al., 2006). Similarly, deletion of Nrp1, a receptor for Pdgf and Fgf that is expressed in the epicardium, affects angiogenesis and epicardial expansion (Lowe et al., 2019). Moreover, a population of $w t 1 b^{+}$macrophages acquires a pro-regenerative profile and is involved in macrophages migration and also in CM proliferation (Sanz-Morejón et al., 2019).

Epicardial cells are also a source of extracellular matrix (ECM) components such as fibronectin, periostin and collagen XII (González-Rosa et al., 2012; Marro et al., 2016; SanzMorejón et al., 2019; Wang et al., 2013). Additionally, fibroblast from the epicardium produce collagen and periostin (Sánchez-Iranzo et al., 2018a), while endocardium is also associated with collagen (Münch et al., 2017). Interestingly, ablation of collagen expressing cells affects CM proliferation, while fibronectin-1 mutants, or the overexpression of a dominant-negative fibronectin fragment do not (Sánchez-Iranzo et al., 2018a; Wang et al., 2013). Additionally, TGF $\beta$ pathway is active during regeneration, since phospho-smad3, a downstream effector of TGF $\beta$, is detected in endocardial, epicardial cells and CM (Chablais and Jazwinska, 2012), and chemical inhibition of TGF $\beta$ decreases ECM deposition and CM proliferation.

Retinoic acid (RA) pathway is also important for CM proliferation (Kikuchi et al., 2011b). Aldh1a2, an enzyme involved in RA synthesis, is expressed in epicardial but also in endocardial cells, suggesting a role for the endocardium on heart regeneration. To that notion, balanced Notch signalling is required for endocardial cell maturation and controlled inflammatory response (Münch et al., 2017). Additionally, Notch is involved in CM proliferation by supressing Wnt signalling in the myocardium, but also by controlling the endocardial availability of Seprin1 (Münch et al., 2017; Zhao et al., 2019).

Angiogenesis is necessary for proper regeneration and has to occur in parallel with the establishment of the new tissue (Marín-Juez et al., 2016). Overexpression of a dominant-negative form of Vegfa abolish vascularization, impairs CM proliferation and arrest regeneration (MarínJuez et al., 2016). Similarly, the heart of the $\operatorname{cxcr} 4$ a mutant that lacks coronary vasculature is unable to regenerate (Harrison et al., 2015).

Surviving CM in the injured heart switch on developmental markers, dedifferentiate and proliferate to populate the injured area (Jopling et al., 2010; Kikuchi et al., 2010). Additionally, both cortical and trabecular myocytes contributing to the newly formed myocardium (Kikuchi et al., 2010; Sánchez-Iranzo et al., 2018b), while, the chemokine Cxcl12a from the epicardium and its receptor Cxcr4b in the CM drive myocytes migration (Itou et al., 2012). Different molecules have been described to affect $\mathrm{CM}$ proliferation. In addition to the aforementioned, inhibition of NF- $\mathrm{KB}$ (Karra et al., 2015) or Erbb/Nrg1 pathways (Gemberling et al., 2015), as well as blockage of Jak1/Stat3 (Fang et al., 2013) or Bmp signalling (Wu et al., 2016) impair CM proliferation and regeneration. Interestingly, deletion of Dusp6, an ERK phosphatase, (Missinato et al., 2018), and 
Yap (Hippo pathway) (Flinn et al., 2019), enhance CM proliferation, similarly to vitamin D treatment (Han et al., 2019).

The dynamic spatio-temporal orchestration of the regeneration programme implicates a tight transcriptional regulation of the multitude of tissue-specific signals and transcription factors involved, that can be achieved through chromatin and histone modifications (Rothbart and Strahl, 2014). For example, Brg1, a chromatin remodelling factor, increases the methylation of $c d k n 1 c$ promoter, and thus represses its transcription resulting in the attenuation of CM proliferation (Xiao et al., 2016). Furthermore, analysis of histone modifications reveals that the epigenetic landscape changes dramatically upon injury (Goldman et al., 2017). For example, H3K27m3 (histone3, lysine27 tri-methylation) is associated with genes suppression and is enriched in sarcomeric and cytoskeletal genes in proliferative CM (Ben-Yair et al., 2019). Additionally, it is possible that specific enhancers get activated only during regeneration, highlighting the importance of cisregulatory elements in regeneration (Kang et al., 2016).

\section{Midkine}

Midkine (Mdk) is a secreted neurite growth factor that was identified as a RA responsive gene in embryonal carcinoma cells (Kadomatsu et al., 1988). Together with Pleiotrophin (Ptn), comprises a family of secreted heparin-binding growth factors (Li et al., 1990). Mdk in the mouse embryo is widely expressed in the brain and spinal cord, but its expression becomes restricted to the kidneys by E16.5 (Fan et al., 2000; Kadomatsu et al., 1990). Detection in the developing heart is moderate, with some weak expression in the valves (Mitsiadis et al., 1995).

Mdk plays multiple roles in neural cells, being required for neurite outgrowth, neuron migration and survival (Michikawa et al., 1993; Muramatsu et al., 1993). However, it is also involved in angiogenesis and leucocytes recruitment. In an artificial blood vessel model of HUVEC and smooth muscle cell (SMC) co-culture, Mdk treatment induces interleukin 8 (Il-8) expression in the SMC, that acts on HUVEC and increases their proliferation (Sumi et al., 2002). Likewise, Mdk stimulates formation of tubular networks in a HUVEC matrigel assay (Takenaka et al., 2009). Additionally, Mdk administration enhances angiogenesis in the chick chorioallantoic membrane (CAM) assay, whereas in the Mdk knock-out (KO) mouse, angiogenesis is impaired after limb injury (Weckbach et al., 2012). Furthermore, Mdk promotes the chemotaxis of macrophages and neutrophils in vitro (Takada et al., 1997) and in vivo (Inoh et al., 2004). During acute inflammation, endothelial Mdk assists neutrophils adhesion. In neutrophils, Mdk facilitates LFA1 (b2 integrin) to acquire a high affinity confirmation for ICAM stable binding, and attachment to endothelial cells (Weckbach et al., 2014).

Mdk-KO mice have no embryonic phenotype but present aberrant behaviour associated to abnormal post-natal development of hippocampus (Nakamura et al., 1998). Moreover, Ptn is highly upregulated in different organs including spinal cord, eye and heart, but not in the brain (Herradon 
et al., 2005). Mdk; Ptn double KO mice are an example of Mdk and Ptn functional redundancy, as a-Tectorin (important for transmission of sound-induced movements in cochlea) is still expressed in the $M d k-K O$ or Ptn-KO, but in the double KO, expression is lost (Zou et al., 2006).

\section{Midkine in cardiovascular diseases and injury}

Mdk expression in adult tissues becomes almost undetectable (Muramatsu et al., 1993) but is upregulated in different types of cancer (Nakagawara et al., 1993) and in neurodegenerative disease (Muramatsul et al., 2011). Furthermore, $M d k$ is induced after injury of different adult tissues such as bone (Haffner-Luntzer et al., 2016), skeletal muscle (Ikutomo et al., 2014), renal (Sato et al., 2001) and heart (Obama et al., 1998). Such disease-associated Mdk induction can be linked to RA and HIF1a, two molecules expressed after disease or trauma [reviewed in (Das et al., 2014; Semenza, 2000)]. That is because Mdk contains RA-responsive and HIF-response elements in the promoter (Matsubara et al., 1994; Reynolds et al., 2004).

The function of Mdk after myocardial infarction has been studied in different animal models including mouse (Horiba et al., 2006; Takenaka et al., 2009), rat (Fukui et al., 2008; Obama et al., 1998; Sumida et al., 2010; Zhao et al., 2014) and pig (Ishiguro et al., 2011), and in all cases $M d k$ is activated. Upon injury, Mdk expression is reported on CM and endothelial cells in both chambers but other authors showed expression only in the $\mathrm{CM}$ of the peri-infarcted zone of rat hearts (Fukui et al., 2008; Obama et al., 1998). Nevertheless, cardiac insult in the $M d k-K O$ mouse leads to larger injury size with increased apoptosis in the periinfarct area and decreased heart function (Horiba et al., 2006). Moreover, treatment of infarcted hearts with exogenous Mdk is beneficial, since it reduces the size of the injured area (Fukui et al., 2008; Horiba et al., 2006), inhibits apoptosis (Sumida et al., 2010; Takenaka et al., 2009; Zhao et al., 2014), and thus improves cardiac function (Fukui et al., 2008; Takenaka et al., 2009; Zhao et al., 2014), and survival of the animals (Takenaka et al., 2009). Similar results are reported also in porcine hearts after ligation of the left anterior descending artery (LAD) injury and ischemia/reperfusion (I/R) with parallel Mdk administration in the infarcted area (Ishiguro et al., 2011). Hence, Mdk administration prevents cardiac remodelling, possibly through decreased collagen deposition and enhanced vascularization. In injured rat hearts treated with Mdk, increased collagen is detected in the infarcted area and reduced only in the non-infarcted area (Fukui et al., 2008). However, longer treatment results in significant smaller collagen deposition, in both the infarcted and healthy areas of the heart (Sumida et al., 2010; Takenaka et al., 2009). Moreover, Mdk administration improves angiogenesis in cardiac tissue upon injury (Fukui et al., 2008; Sumida et al., 2010; Takenaka et al., 2009). This is attributed to increased expression of Syndecans (-1, -3 and -4) and HIF1a as well as in activation of the PI3-kinase/Akt and ERK1/2 pathways that regulate expression of angiogenesis-related genes (Sumida et al., 2010; Takenaka et al., 2009). Additionally, these phosphorylation cascades contribute to $\mathrm{CM}$ survival, since $\mathrm{Mdk}$ administration in cultured myocytes with 
hypoxia/reoxygenation injury induces expression of the antiapoptotic factor Bcl2 and ERK1/2 phosphorylation, decreasing cell death (Horiba et al., 2006).

Although Mdk has a beneficial effect after heart injury, it can also have adverse results. Heart-specific Mdk overexpression results in increased fibrosis, and ERK1/2 and AKT phosphorylation, that progressively leads to cardiac hypertrophy and dysfunction after transverse aortic constriction (TAC - a model of pressure overload) (Netsu et al., 2014). Additionally, TAC results in Mdk expression in the kidney and the lung that may contribute to the phenotype (Netsu et al., 2014). Hence, secreted Midkine may be associated with cardio-renal interaction. On this regard, nephrectomy increases Mdk concentration in the circulation, leading to EGFR autophosphorylation, and to activation of ERK1/2 and AKT phosphorylation cascades, contributing to cardiac dysfunction (Honda et al., 2016). Additionally, Mdk concentration in the plasma is increased after multiple injuries in humans and pigs and affects post-trauma cardiac defects. Mdk acts directly on CM, since Mdk treatment in culture human CM alters gene expression of calcium handling molecules, and decreases the mitochondrial respiratory capacity of the cells (Lackner, Weber et al., 2019). Furthermore, Mdk is involved in the progress of myocarditis (chronic inflammatory cardiomyopathy). Mdk association with neutrophils adhesion leads to increases cardiac inflammation, while inhibition of Mdk decreases neutrophils infiltration and reduces cardiac fibrosis (Weckbach et al., 2019). Moreover, increased Mdk expression is detected in neointima of mouse carotid after balloon injury (Horiba et al., 2000). In the $M d k-K O$ mouse, neointima is repressed while administration of Mdk restores neointima progression. That is linked to impaired infiltration of leukocytes since Mdk treatment in wild type mouse increases the infiltration of macrophages and neutrophils. Similarly, suppression of Mdk represses neointima thickening of the aorta (Hayashi et al., 2005) and of the vein grafts in rabbits (Banno et al., 2006). On this regard, $M d k$ is upregulated in a mouse bleomycin-induced pulmonary fibrosis model (Misa et al., 2017), and deletion of $M d k$ results in reduction of fibrosis, due to impaired lymphocyte migration and decreased TNF $\alpha$ and TGF $\beta$ expression.

Overall, Mdk has a protective effect against ventricular and vascular remodelling after injury, through the activation of angiogenesis and leucocytes recruitment, although uncontrolled availability of Mdk can have deleterious effects.

\section{Midkine in zebrafish}

In zebrafish, Midkine-a (Mdka) was identified after a screening for neural inducing factors (Winkler et al., 2003). Mdka and its paralogue Midkine-b (Mdkb), are the product of the fishspecific genome duplication, having complementary patterns of expression in neural tube during early development (16hpf) (Winkler et al., 2003). Together with Ptn, they comprise a small protein family of neurite outgrowth cytokines in zebrafish (Chang et al., 2004). The onset of $m d k a$ expression is $8 \mathrm{hpf}$ in the paraxial mesoderm and the head, whereas later is expressed in the neural 
ectoderm of the spinal tube, in retina and the brain (Winkler et al., 2003). In adults, Mdka is still detectable in the retina and the brain (Gramage et al., 2015; Schäfer et al., 2005; Winkler et al., 2003). In embryos, Mdka overexpression results in expansion of the media floor plate (MFP) (Schäfer et al., 2005), a structure in the ventral site of neural tube involved in neurons specification and organization (Halpern et al., 1997), as well as in disturbed somite boundaries and retina overgrowth (Luo et al., 2012; Winkler et al., 2003). Mdka loss-of-function (morphants) results in reduced floor plate, decreased retina proliferation and microphthalmia (Luo et al., 2012; Schäfer et al., 2005). Furthermore, $m d k a$ is expressed in adult retina in horizontal cells of the adult retina, and it is induced after photolytic lesion in Muller glia and proliferating photoreceptor progenitors (Calinescu et al., 2009a; Gramage et al., 2015). Reduction of $m d k a$ during retina regeneration leads to decreased proliferation and reduced rod photoreceptors (Gramage et al., 2015).

\section{Caveolae and Caveolin-1}

The plasma membrane of cells consists of microdomains with different composition of lipids and proteins that are important for signal transduction (Fielding and Fielding, 2004). In that regard, small membrane invagination having omega $(\Omega)$ shape and resembling intracellular 'caves' were first reported in electron microscopy studies of epithelial cells and were named caveolae from the Latin word for caves (Figure 3A-D) (Palade, 1953; Yamada, 1955). Characterization of these membrane domains was accelerated after the discovery of CAVEOLIN-1 (CAV1 or VPN21), an integral membrane protein (Kurzchalia et al., 1992; Rothberg et al., 1992) that was proven to be the main structural protein for caveolae. CAV1 overexpression in cells lacking caveolae induces formation of caveolae-like structures, while reduction of CAV1 leads to caveolae depletion (Fra et al., 1995; Koleske et al., 1995). Since then, the caveolae-related gene family has grown with the identification of two more Caveolins, CAVEOLIN-2 (CAV2) and CAVEOLIN-3 (CAV3) (Scherer et al., 1996; Way and Parton, 1995) and the Cavin protein family including CAVIN-1 (or PTRF), CAVIN-2 (or SDPR), CAVIN-3 (or SRBC) and CAVIN-4 (or MURC) (Bastiani et al., 2009; Hansen et al., 2009; Hill et al., 2008; Liu et al., 2008; McMahon et al., 2009). CAV1, CAV3 and CAVIN1 are essential for caveolae formation, because genetic deletion leads to loss of caveolae (Drab et al., 2001; Galbiati et al., 2001; Liu et al., 2008; Razani et al., 2001a; Zhao et al., 2002). In contrast, CAV2, CAVIN-2, -3 and -4 are considered accessory proteins, since their loss does not affect caveolae formation (Hansen et al., 2013; Ogata et al., 2014; Razani et al., 2002).

\section{Caveolins expression, structure and caveolae assembly}

Caveolae are abundant in different cell types while Caveolin's distribution varies. CAV1 is strongly expressed in endothelia, epithelial cells and fibroblasts, and is more weakly expressed in myocytes (Cohen et al., 2003; Mora et al., 1999; Patel et al., 2007; Robenek et al., 2008a; Scherer et al., 1997). CAV2 has the same expression pattern than CAV1, and the stability of CAV2 depends 
on CAV1 (Mora et al., 1999; Razani et al., 2001a). In contrast, CAV3 is specific of skeletal and cardiac muscle cells (Tang et al., 1996) while CAVIN1 has been detected in a variety of cells, including epithelial and endothelial cells, as well as in cardiomyocytes (CM) (Hansen et al., 2013). Interestingly, CAVIN1 localizes also to the nucleus, and is involved in transcriptional regulation (Bai et al., 2011; Hasegawa et al., 2000).

CAV1 structure and domain organization directly reflects caveolae assemble. Both $\mathrm{N}$ - and C-terminus face the cytosol, with the middle part displaying a hairpin structure, inserted into the lipid bilayer leading to membrane curvature (Figure 3E-F) [reviewed in (Parton et al., 2006)]. The caveolin scaffolding domain (CSD) at N-terminus facilitates cholesterol recognition, protein interaction, membrane binding and CAV1 oligomerization (Couet et al., 1997; Schlegel et al., 1999), while the C-terminus is important for CAV1 oligomer interaction, intercellular trafficking and membrane attachment, having three conserved cysteines that can be palmitoylated (Monier et al., 1996; Ren, 2004; Schlegel and Lisanti, 2000; Song et al., 1997). CAVIN1 is also involved in caveolae morphogenesis by forming oligomers that interact with CAV1 and covers the surface of caveolae (Stoeber et al., 2016).

\section{The role of caveolae and CAV1}

Caveolae significance increased once studies revealed that signalling molecules and receptor localize in these membrane microdomains. Caveolae are involved in various processes, including cholesterol homoeostasis, signal transduction and mechanoprotection (Cheng et al., 2015; Cohen et al., 2003; Kim et al., 2008a). For mechanoprotection, caveolae deliver the extra membrane that cells need to buffer the impact of mechanical forces, through rapid disassemble and flattening of caveolae (Gervásio et al., 2011; Sinha et al., 2011). Physiologically, caveolae protect mouse cardiac endothelial cells from rupture due to increased cardiac output (Cheng et al., 2015), and in zebrafish, caveolae protect from damage in skeletal muscles after vigorous motion (Lo et al., 2015), and also preserve notochord integrity (Garcia et al., 2017; Lim et al., 2017). Additionally, caveolae regulate signal transduction via CAVEOLIN-1 direct interaction with signalling molecules, including endothelial nitric oxide synthase (eNOS) and TGF $\beta$ signalling. In particular, CAV1 negatively regulate the nitric oxides (NO) availability by binding and blocking eNOS that reside in caveolae (García-Cardeña et al., 1996). Additionally, CAV1 interact directly with TGFßR1, blocking Smad complex nuclear translocation and thus inhibiting transcriptional activation (Razani et al., 2001b). 

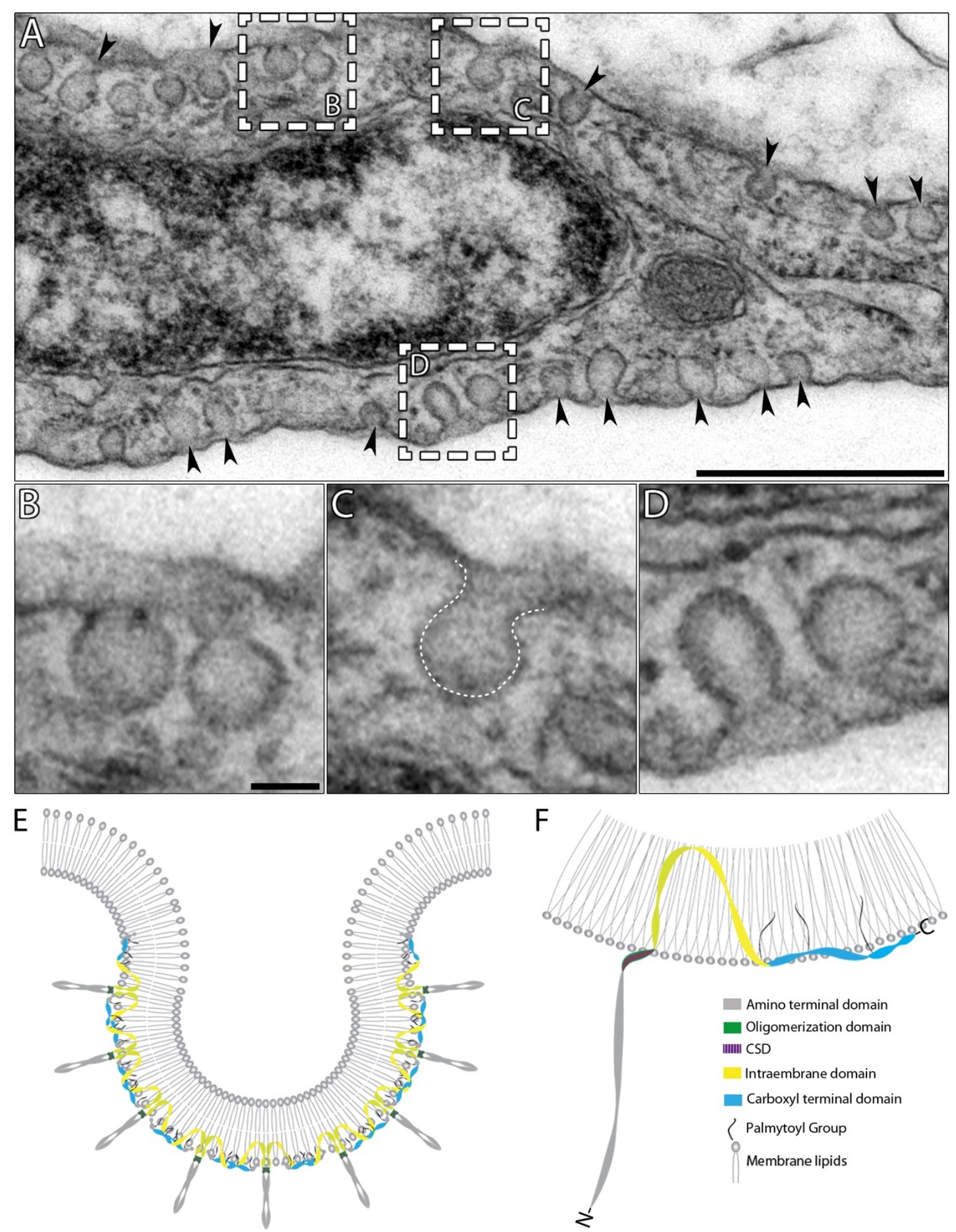

Figure 3. Caveolae and Caveolin-1 domain organization

(A) Transmission electron microscopy images of epicardial cell from adult zebrafish heart, arrowheads indicate caveolae. (B-D) Higher magnification of selected areas in A. (C) Individual caveolae with the dashed line following the omega $(\Omega)$ shape. Scale bars, A $500 \mathrm{~nm}, \mathrm{~B}, \mathrm{C}$ and D 50nm C. (E) Representation of membrane bound Cav1 oligomers on caveolae microdomain. (F) Caveolin-1 domain organization. 


\section{The role of CAV1 in the mammalian heart}

Various Cav1-KO animal models have been generated having diverse cardiovascular phenotypes. Deletion of $\mathrm{Cavl}$ in mouse results in adverse cardiac remodelling including right ventricle dilatation and left ventricle hypertrophy secondary to pulmonary hypertension (Cohen et al., 2003; Park et al., 2003; Zhao et al., 2002). These phenotypes are attributed to increased fibrosis in the heart and to the endothelial loss of CAV1 that leads to eNOS hyperactivation. (Cohen et al., 2003; Drab et al., 2001; Murata et al., 2007; Park et al., 2003). Endothelial-specific expression of CAV1 in Cavl-KO mice decreases pulmonary pressure, normalizes interstitial fibrosis and rescues remodelling of the chambers (Murata et al., 2007). Additionally, Cav1-KO mice present defects in heart function, including decreased systolic and diastolic function (Cohen et al., 2003; Park et al., 2003; Wunderlich et al., 2006; Zhao et al., 2002) that are exacerbated after myocardial infarction (Jasmin et al., 2011; Shivshankar et al., 2014). Cardiac insult also leads to aberrant fibrosis thought increased Smad2/3 phosphorylation and M2 macrophages infiltration in Cav1-KO mice (Miyasato et al., 2011; Shivshankar et al., 2014).

\section{Caveolin-1 in zebrafish}

In zebrafish, cavl expressed begins early in embryogenesis showing a broad expression pattern, including the notochord, pharyngeal vasculature and heart (Fang et al., 2006). Morpholino experiments revealed the importance of cavl in lateral line neuromast and notochord formation (Nixon et al., 2007). To date, all Caveolins and Cavins have been identified in zebrafish, except Cavin-3 (Hill et al., 2008; Housley et al., 2016; Lo et al., 2015; Nixon et al., 2005) with the addition of Cavy that initially was predicted in an evolutionary comparative study and was later identified (Kirkham et al., 2008; Lim et al., 2017). A few zebrafish caveolae-related gene knock-out models have been generated including cavin1, cavin4 and cavl (Cao et al., 2016; Garcia et al., 2017; Housley et al., 2016; Lim et al., 2017). Interestingly, KO of cavin1 does not lead to loss of caveolae but to a reduction in numbers and abnormal morphology (Lim et al., 2017; Liu et al., 2008), whereas, in the cav1-KO zebrafish the caveolae status is not addressed (Cao et al., 2016). Remarkably, deletion of the muscle-specific cavin 4 results in increased caveolae number in skeletal muscle cells (Housley et al., 2016). However, loss of Cavin1b results in traumas in the notochord upon rapid movement of the larva, whereas cavin $4 b-K O$ embryos develop fibrosis in the skeletal muscle and T-tubule abnormalities. Cardiac regeneration has been studied in the cavl-KO fish, where ventricular amputation leads to increased fibrosis. Although, CAV1 negatively regulated TGF $\beta$ (Razani et al., 2001b), they did not find increase of TGF $\beta$ signalling activity. Additionally, they reported decreased CM proliferation and arrest of the regeneration 30-days post injury (Cao et al., 2016). 


\section{Midkine-a and Caveolin-1}

In this thesis we have studied the roles of midkine-a (mdka) and caveolin-1 (cavl) in heart regeneration, two genes that were found upregulated in a microarray analysis of regenerating hearts performed in the laboratory (González Rajal, 2012). Additionally, we have analysed the epigenetic regulation of $m d k a$ and test in vivo cis-regulatory elements of $m d k a$ locus during development and in regeneration.

Here, we have generated $m d k a-K O$ zebrafish strain to investigate of the role of Mdka during heart regeneration. We show that loss of $m d k a$ arrests heart regeneration 90dpci, since the KO hearts have a significant larger injury, characterized by extensive collagen deposition. Moreover, we have studied the epigenetic regulation of $m d k a$ and tested in vivo cis-regulatory elements. We have found that there are five enhancers in $m d k a$ locus, and that the three intronic enhancers with the promoter of $m d k a$ are responsible for the dynamic expression of $m d k a$ during development, in adult tissues and after heart and fin injury.

Furthermore, we have investigated the importance of Caveolin-1 and caveolae for the maintenance of the mechanical properties of the cardiac tissue and in heart regeneration. To this end, we have generated a series of cavl-KO using CRISPR/Cas9 gene editing and have used the cryoinjury model to assess the role of Cav1 in heart regeneration. This model results in the formation of a transient scar tissue (Chablais et al., 2011; Gonzalez-Rosa et al., 2011; Schnabel et al., 2011) that resolves 90-day post cryoinjury (dpci). We have found that Cav1 and caveolae depletion do not affect heart regeneration but lead to a transient decrease in CM proliferation. Using atomic force microscopy (AFM) we have detected a significant difference in cardiac elasticity. Hearts lacking caveolae, were stiffer compared to control hearts a phenotype that was accompanied by cardiac dysfunction, highlighting the role of caveolae in the maintenance of the mechanical properties of the heart. 


\section{Objectives}


This doctoral thesis aims at understanding the molecular and mechanophysiological properties of the control and regenerating zebrafish heart. For this purpose, we have generated knock-out zebrafish strains using CRISPR/Cas9 genetic editing for midkine-a (mdka) and caveolin-1 (cavl), two genes with different characteristics and properties that are upregulated upon heart injury. Furthermore, we have studied and tested in vivo the epigenetic regulatory elements of $m d k a$ locus due to the highly dynamic expression of this gene during development and regeneration. The main objectives of this study are:

- Investigate the role of $m d k a$ during heart regeneration

- Dissect and investigate the epigenetic landscape of $m d k a$

- Identify the minimum cis-regulatory elements that drive $m d k a$ expression in development and regeneration

- Study the role of cavl in regenerating hearts

- Study the impact of cavl loss in heart function 
Materials and Methods 


\section{Zebrafish husbandry and transgenic lines}

Zebrafish were raised under standard conditions at $28^{\circ} \mathrm{C}$ as described in Kimmel et al., 1995. Experiments were performed with $1 \mathrm{dpf}$ to $6 \mathrm{dpf}$ embryos and with 6-weeks to 13-month-old adults. All zebrafish strains used or generated during this thesis are summarized in table 1.

Table 1. Zebrafish strains used in this thesis

\begin{tabular}{|c|c|c|c|}
\hline Strain & Short name & Reference & Description \\
\hline AB & wild type (WT) & & wild type zebrafish \\
\hline $\operatorname{cav1} 1^{+/}$ & & this thesis & caveolin-1 KO \\
\hline $\operatorname{cav1a}{ }^{+/-}$ & & this thesis & caveolin-1a KO \\
\hline $\begin{array}{l}\text { Tg(FRT-Xla.Actc1:DsRed- } \\
\text { GAB-FRT,LOXP- +2mdka } \\
\text {-LOXP-gata2a:EGFP- } \\
\text { 5HS4) }\end{array}$ & $\operatorname{Tg}(e n h: G F P)$ & this thesis & $\begin{array}{l}\text { enh genomic region of } m d k a \text { locus } \\
\text { upstream of the gata } 2 \text { promoter } \\
\text { driving the expression of GFP }\end{array}$ \\
\hline $\begin{array}{l}\text { Tg(FRT-Xla.Actc1:DsRed- } \\
\text { GAB-FRT,LOXP--4.8mdka } \\
\text {-LOXP-gata2a:EGFP- } \\
\text { 5HS4) }\end{array}$ & $\operatorname{Tg}($ enha4:GFP) & this thesis & $\begin{array}{l}\text { enha4 genomic region of } m d k a \text { locus } \\
\text { upstream of the gata } 2 \text { promoter } \\
\text { driving the expression of GFP }\end{array}$ \\
\hline $\operatorname{Tg}(-2.5 m d k a-m d k a: e G F P)$ & $\operatorname{Tg}($ enha5:GFP) & this thesis & $\begin{array}{l}\text { enha5 genomic region of } m d k a \text { locus } \\
\text { upstream of the } m d k a \text { promoter } \\
\text { driving the expression of GFP }\end{array}$ \\
\hline $\begin{array}{l}\text { Tg(FRT-Xla.Actc1:DsRed- } \\
\text { GAB-FRT,LOXP- } \\
+5.8 m d k a-L O X P- \\
\text { gata2a:EGFP-5HS4) }\end{array}$ & $\operatorname{Tg}(\operatorname{Reg}: G F P)$ & this thesis & $\begin{array}{l}\text { Reg genomic region of } m d k a \text { locus } \\
\text { upstream of the gata } 2 \text { promoter } \\
\text { driving the expression of GFP }\end{array}$ \\
\hline $\begin{array}{l}\text { Tg(FRT-Xla.Actc1:DsRed- } \\
\text { GAB-FRT,LOXP-mdka- } \\
\text { LOXP-gata2a:EGFP- } \\
\text { 5HS4) }\end{array}$ & $\operatorname{Tg}(m d k a: G F P)$ & this thesis & $\begin{array}{l}m d k a \text { promoter upstream of the gata } 2 \\
\text { promoter drives GFP expression }\end{array}$ \\
\hline$T g(+2 m d k a-m d k a: e G F P)$ & $\begin{array}{l}\operatorname{Tg}(e n h- \\
m d k a: G F P)\end{array}$ & this thesis & $\begin{array}{l}\text { enh region upstream of the } m d k a \\
\text { promoter drives GFP expression }\end{array}$ \\
\hline$m d k a^{+/-}$ & & this thesis & $m d k a K O$ \\
\hline $\operatorname{Tg}($ flila:GFP) & & $\begin{array}{l}\text { (Lawson and } \\
\text { Weinstein, } \\
\text { 2002) }\end{array}$ & $\begin{array}{l}\text { GFP expression in the endothelium } \\
\text { and in the endocardium }\end{array}$ \\
\hline
\end{tabular}




\begin{tabular}{|c|c|c|}
\hline Tg(fli1a:Redex) & $\begin{array}{l}\text { (Covassin et } \\
\text { al., 2009) }\end{array}$ & $\begin{array}{l}\text { RFP expression in the endothelium } \\
\text { and in the endocardium }\end{array}$ \\
\hline $\operatorname{Tg}(m y l 7: m R F P)$ & $\begin{array}{l}\text { (Rohr et al., } \\
2008)\end{array}$ & $\begin{array}{l}\text { membrane bound GFP expressed in } \\
\text { cardiomyocytes }\end{array}$ \\
\hline$T g(w t 1 b: G F P)$ & $\begin{array}{l}\text { (Perner et al., } \\
2007)\end{array}$ & $\begin{array}{l}\text { GFP expression in the epicardium of } \\
\text { the adult heart upon injury }\end{array}$ \\
\hline
\end{tabular}

\section{Generation of the transgenic line}

All $m d k a$ regulatory elements (mdka, enh, enha4, enh5 and Reg) were PCR amplified (Table 2) and ligated to the pCR8 vector by TA cloning (Invitrogen). The resulting plasmids were then inserted into the ZED (Bessa et al., 2009) or the pminiTol2 vectors by LR Clonase II recombination (Thermo Fisher Scientific). In the case of mdka-hne, the enh genomic region was PCR amplified by primers with restriction enzyme (NotI and BmgBI) sites and was ligated in reverse orientation into the pCR8-mdka plasmid 3prime of the mdka promoter and then to the pminiTol2 vector by Gateway recombination. We used the crispr.mit.edu tool (https://zlab.bio/guide-design-resources) to design the guides for $m d k a$ and $c a v 1$, selecting the guides with the less intronic off-targets. The oligos were annealed, phosphorylated and ligated according to the published protocol (Ann, F et al., 2013) to the pX330-U6-Chimeric_BB-CBhhSpCas9 vector (\#42230 Addgene) (Cong et al., 2013) that was linearized by the BbsI enzyme (New England Biolabs). Primers with the T7 polymerase promoter-specific sequences were used to PCR amplify the guide RNA (crRNA and tracrRNA). The MEGAshortscript T7 Transcription Kit (Thermo Fisher Scientific) was used for guide RNA transcription that was purified by the NucAway Spin Columns kit (Thermo Fisher Scientific).

Table 2. Primer list for plasmid generation

\begin{tabular}{|l|l|}
\hline Name & Sequence \\
\hline enh Fwd & GCTTCATTCACTCTCTCTCTTTC \\
\hline enh Rvs & AAGCTACATTAAGCATTCTATGG \\
\hline enha4 Fwd & CTACAGAAATTATGAATCCCA \\
\hline enha4 Rvs & CCTCAACTTCAAATATCCCAA \\
\hline enha5 Fwd & TGTCGGCTTAATATCTGCT \\
\hline enha5 Rvs & CTTATTTATCAGGGATCACCAC \\
\hline Reg Fwd & GGGCCTCAGCGTTACTTTAAGG \\
\hline Reg Rvs & GGGGACGTGCTCTTTTCAATCATGC \\
\hline mdka Fwd & TTGTTTTCGCGCAACATGAT \\
\hline mdka Rvs & TTTCCCCTCAGCAGTCCCATT \\
\hline
\end{tabular}




\begin{tabular}{|c|c|}
\hline enh BmgBI Fwd & GCGCGCCACGTCGCTTCATTCACTCTCTCTCTTTCC \\
\hline enh NotI Rvs & ATAAGAATGCGGCCGCAAGCTACATTAAGCATTC \\
\hline$m d k a$ top & CACCATAGAGCCACTCCGCACAGT \\
\hline mdka bot & AAACACTGTGCGGAGTGGCTCTAT \\
\hline mdka T7 & $\begin{array}{l}\text { ACGGGGTAATACGACTCACTATAGGGATAGAGCCACTCCGCA } \\
\text { CAG }\end{array}$ \\
\hline cav1 guide 2 top & CACCGGCGAGTGAGCGTATTCCTG \\
\hline cav1 guide 2 bot & AAACCAGGAATACGCTCACTCGCC \\
\hline cav1 guide 2 T7 & ACGGGGTAATACGACTCACTATAGGCGAGTGAGCGTATTCC \\
\hline cav1 guide 1 top & CACCGGTGGGCATCCCACTCGCCC \\
\hline cav1 guide 1 bot & AAACGGGCGAGTGGGATGCCCACC \\
\hline cav1 guide 1 T7 & TAATACGACTCACTATAGGTGGGCATCCCA \\
\hline tracrRNA Rvs & AAAAGCACCGACTCGGTGCCAC \\
\hline
\end{tabular}

\section{Microinjection of DNA and RNA}

The injection mix for the generation of the transgenic lines contained plasmid DNA and transposases mRNA at final concentration of $25-50 \mathrm{ng} / \mu \mathrm{l}$ and $200 \mathrm{ng} / \mu \mathrm{l}$, respectively. The DNA constructs and mRNAs were microinjected into the yoke ventrally to the cell of one-cell stage zebrafish embryos using the Narishige IM 300 microinjector. All injected embryos were sorted and selected by fluorescence expression 1dpf. For the CRISPR/Cas9 injections, the guide RNA (200 $\mathrm{ng} / \mu \mathrm{l})$ together with the Cas 9 protein $(0,5 \mu \mathrm{M}$, New England Biolabs $)$ were injected to the blastodisc of one-cell stage zebrafish embryos. The injected embryos were genotyped (Table3) 1 dpf to examine if they were carrying indels in the target locus and then the embryos were let to grow.

Table 3. Primers for genotyping the CRISPR/Cas9 lines

\begin{tabular}{|l|l|}
\hline Name & Sequence \\
\hline cav1 Fwd & GGCGAGCTTCACCACCTTC \\
\hline cav1 Rvs & GCTCTTCACGCAAGGCACCA \\
\hline cav1 $\boldsymbol{a}$ Fwd & TGGTGCTTCATTTCTATCCG \\
\hline $\boldsymbol{c a v 1} \boldsymbol{a}$ Rvs & TAAATTTAAATTGAATACCCAAC \\
\hline mdka Fwd & TGTTATGTATGATTCTGCGAT \\
\hline mak $\boldsymbol{a}$ Rvs & ACAGAGGCACAAAACTACCAA \\
\hline
\end{tabular}

\section{Fin amputation}

The amputation of the caudal fin for DNA and protein extraction was performed as described before (Akimenko et al., 1995). Briefly, the fish were anesthetized by $0,04 \%$ tricaine 
(Aldrich) and half of the caudal fin was amputated using a scalpel. The fish were let to recover in fish water before returned to their tanks.

\section{Cryoinjury of the adult heart}

The fish were anesthetized by $0,04 \%$ tricaine and placed on a wet sponge with the ventral site exposed under a stereoscope. Using microscissors and microforceps the cardiac cavity was opened and the pericardium was removed. The ventricle of the heart was exposed and dried and then was touched for $10 \mathrm{sec}$ by a coper-made probe that was immersed in liquid nitrogen (GonzálezRosa and Mercader, 2012). The fish were immediately returned to water to recover and then were moved to their tanks.

\section{Bromodeoxyuridine (5-bromo-2'-deoxyuridine - BrdU) injection}

Adult fish were anesthetized and placed on a wet sponge under a stereoscope. The BrdU was diluted in Phosphate-Buffered Saline (PBS) to $2,5 \mathrm{mg} / \mathrm{ml}$ and $30 \mu \mathrm{l}$ were injected intraperitoneally. The fish then were transferred to their tanks.

\section{Echocardiography}

Analysis of the cardiac function by echocardiography in adult fish was performed as described in (González-Rosa et al., 2014). Briefly, the fish were anesthetized by $60 \mathrm{mM}$ tricaine and $3 \mathrm{mM}$ isoflurane in fish water and transferred to a sponge immersed in the same solution. The VEVO 2100 system (VisualSonics) that has a 50MHz ultrasound probe was used for the measurement. The transductor was immersed in the medium dorsally to the cardiac cavity. After the procedure was finished, the fish were immediately transferred to fresh water to recover. The procedure lasted approximately 5 min per fish and the anaesthesia solution was refreshed every 4 fish.

\section{Atomic force microscopy (AFM)}

Adult zebrafish were sacrificed by $0,16 \%$ tricaine and the heart was dissected. The atrium was removed and the ventricle was placed horizontally on top of a 3\% agarose gel immersed in PBS with $0,1 \mathrm{M} \mathrm{KCl}$. The AFM experiments were performed with a JPK Nanowizard III (JPK Instruments) mounted on an inverted optical microscope (AXIO Observer D1, Carl Zeiss). We used Plateau-CONT-SPL cantilevers (NANOSENSORSTM) with a nominal spring constant of 0,02$0,77 \mathrm{~N} / \mathrm{m}$ and a spherical tip shape $(\mathrm{R}=30 \mu \mathrm{m})$. The actual spring constant of the cantilever was determined using the thermal noise method as implemented in the AFM software. Force-distance curves were acquired to determine the Young's modulus of the cardiac apex. The tip-sample distance was modulated by applying a triangular waveform with the tip velocity set at $10 \mu \mathrm{m} / \mathrm{s}$. The maximum force exerted on the apex of the heart during a single FDC was of $11 \mathrm{nN}$. For each sample, a complete sequence of 375 FDC was performed. These FDC were distributed in three different 
areas of $100 \times 100 \mu \mathrm{m}^{2}$ separated by approximately hundreds of microns (125 FDC were obtained per zone), all along the cardiac apex. To determine the contact point, we used a ratio of variances protocol. The Young's modulus was obtained by fitting a section of the force-distance curve (approach semi cycle of the whole FDC) with a Hertz model for spherical indenters.

\section{Western blot (WB)}

Protein expression analysis by WB was performed in hearts (pools of three or five hearts) or embryos (pools of 20 or 50 embryos). All samples were lysed in lysis buffer $(150 \mathrm{mM} \mathrm{NaCl}$, $25 \mathrm{mM}$ Tris $\mathrm{pH} 7,5,1,5 \mathrm{mM} \mathrm{MgCl} 2,1 \%$ Triton $\mathrm{X}-100,10 \mathrm{mM}$ DTT, phosphatase and protease inhibitors) or in Ripa buffer (25mM Tris $\mathrm{pH} 7,5,150 \mathrm{mM} \mathrm{NaCl}, 0,5 \% \mathrm{NP} 40,0,5 \% \mathrm{SDS}$ and protease and phosphatase inhibitors). Samples were sonicated (NESLAB RTE 7) and stored at $-80^{\circ} \mathrm{C}$ upon use. Before SDS-page electrophoresis, all samples were boiled for $5 \mathrm{~min}$ and equal amount of proteins (30mg) was loaded. Proteins were transferred to PVDF Immobilon-P (Millipore) at $4^{\circ} \mathrm{C}$ and after the membrane was blocked with $5 \%$ milk in Tris-Buffered Saline (TBS)-Tween $0,1 \%$ for $1 \mathrm{~h}$. The primary antibody was diluted in TBS-Tween $0,1 \% / 2 \%$ BSA and membranes were incubated overnight $(\mathrm{O} / \mathrm{N})$ at $4^{\circ} \mathrm{C}$ (Table 5). Membranes were washed with TBS-Tween $0,1 \%$ before incubation with the secondary antibody coupled to horseradish peroxidase (Dako Cytomation). Finally, membranes were incubated with the Immobilon Western HRP substrate (Millipore) for $1 \mathrm{~min}$ and exposed to the film for $10 \mathrm{sec}, 30 \mathrm{sec}, 1 \mathrm{~min}, 5 \mathrm{~min}$ and $10 \mathrm{~min}$. The films were developed by the KODAK Medical X-Ray Processor 102 processor. Alternatively, after the ECL incubation, membranes were imaged with the Image Quant LAS 4000 mini machine.

\section{Analysis of transcription factor binding sites}

All $m d k a$ cis-regulatory elements were submitted for analysis in the Jaspar database (jaspar.genereg.net). We selected the top-10 hits and their matrixes were reanalysed and refined by the meme server (http://meme-suite.org/).

\section{In silico modelling of the Cav1 mutants}

To analyse the putative membrane domain of the mutant forms Cav1 protein, all sequences were submitted to several predictor servers: octopus (http://octopus.cbr.su.se/), topcons2 (http://topcons.net/) and TMHMM2 (http://www.cbs.dtu.dk/). These methods are optimized for predict transmembrane domains but they can find insertions too (TMHMM worse). The sequences were also submitted to a local implementation of Rosetta software suite v3.8 (www.rosettacommons.org) (Barth et al., 2009) for ab-initio modeling using the mp_framework of the suite. The best model with minimal energy and correct folding (compatible with insertion or deep embedded in membrane) was selected as final template for refinement using the relax tool (Conway et al., 2014; Nivón et al., 2013) of Rosetta suite v3.8 (www.rosettacommons.org). The 
model with minimal energy was selected as final model. To better show the protein position and orientation in the membrane, the model before was submitted to the PPM server (http://opm.phar.umich.edu/). This server is specialized in predict and positioning membrane proteins from 3D structures using a large structural database (membranome) and computational methods. This server predicts an insertion or deep embedded membrane domain. For docking the dimeric form of homo and heterodimers for Cav1 mutants, combinations of two monomers models modelled before were positioned according to the dimeric interface for Cav1 previously published in bibliography using the pymol v1.8.9 program (Schrödinger, 2015) and each new dimeric model was used as initial template. For each template, a span file with coordinates for the theoretical membrane domain was computed using the mp_span_from_pdb tool from the membrane framework of Rosetta suite v3.8 (www.rosettacommons.org), the obtained results were manually curated and modified as necessary. In each dimer, using the complexes templates and span-files obtained before, a primary cycle of docking without positional restrictions using the mp_dock application (Alford et al., 2015) from the membrane framework of Rosetta suite v3.8 (www.rosettacommons.org) and structural clustering was made. For each case, the best model with minimal E compatible with membrane topology and close to the theoretical dimer interface was selected. A final cycle of relax for minimize $\mathrm{E}$ and clashes using the model before as template and the full spanfile was made using a new cycle of docking with positional restrictions (dimer interface) using the mp_dock application from the Rosetta suite v3.8 (www.rosettacommons.org) (Alford et al., 2015). The lowest scoring refined model (lower E) with correct topology and interface was selected in each case as final model. To better show the dimer position and orientation in the membrane, the models before were submitted to the PPM server (http://opm.phar.umich.edu/). This server is specialized in predict and positioning membrane proteins from 3D structures using a large structural database (membranome) and computational methods.

\section{Riboprobe generation}

Specific exonic regions that span between two exons were chosen for riboprobe generation and were amplified by PCR with specific primes (Table4) using as template cDNA from hearts or embryos. The PCR product was ligated to the pGEM-T easy vector (Promega) and DH5a bacterial cells were transfected with the resulting plasmids. Positive colonies were identified by colony-PCR and grew $\mathrm{O} / \mathrm{N}$ in LB medium. We used the Plasmid Maxi Kit (Quiagen) to isolate the plasmids that were confirmed by sequencing. The plasmids were linearized by the appropriate enzyme and riboprobes were transcribed by either $\mathrm{T} 7$ or $\mathrm{Sp} 6$ polymerase $\left(2 \mathrm{~h}\right.$ at $\left.37^{\circ} \mathrm{C}\right)$. The RNA probes were purified by the Illustra AutoSeq G-50 Kit (GA healthcare) and stored at $-80^{\circ} \mathrm{C}$. 
Table 4. Primers for RNA probe

\begin{tabular}{|c|c|}
\hline Name & Sequence \\
\hline cav1 Fwd & ATGACGATGTGGTGAAGGTG \\
\hline cav1 Rvs & ACAGTATTCCTACAATGATAA \\
\hline$m$ Cav1 Fwd & CAAGCATCTCAACGACGAC \\
\hline$m$ Cav1 Rvs & CCCTAAACAAATGGCCGTA \\
\hline mdka Fwd & CAAAGGAAAGAAAGGCAAGGGGAAAGGGAACTAAAGG \\
\hline mdka Rvs & GACTTTCCAATTGCTACTTTTATACCCCGCTAGTAAT \\
\hline mdkb Fwd & CCCAATAGCGGAGACTGT \\
\hline mdkb Rvs & ATCTTGCCAGTTTTCCTCT \\
\hline ptn Fwd & CAGGAATGAAGACTCGCACT \\
\hline ptn Rvs & GCTGCATAAATCAATGACCA \\
\hline
\end{tabular}

\section{Tissues dissection and process}

Adult fish were sacrificed by transfer to fish water containing $0,16 \%$ tricaine, sodium heparin (1,000 UI/ml, Laboratorios Farmacéuticos ROVI) and 0,1M KCl (González-Rosa and Mercader, 2012). Embryos were sacrificed in iced-water and tricaine. For in vivo imaging, fins or embryos were fixed in 2\% paraformaldehyde solution (PFA) and washed with PBS before analysis. Hearts and brains were surgically removed and fixed overnight $(\mathrm{O} / \mathrm{N})$ with $4 \%$ PFA in PBS- $0.1 \%$ tween20 similar to the embryos and the fins. After fixation, tissues and embryos were washes in PBS- $0.1 \%$ tween 20 . For embedding in paraffin, a series of EtOH washes $(50 \%, 70 \%, 80 \%, 90 \%$, $95 \%$, and twice in $100 \% \mathrm{EtOH}$ ) was followed to dehydrate the tissues before xylene washes that followed by incubation in paraffin at $65^{\circ} \mathrm{C}$. For gelatin-embed, tissues were washed in PBS- $0.1 \%$ tween 20 after fixation and then were incubated with $15 \%$ sucrose at $4^{\circ} \mathrm{C} \mathrm{O} / \mathrm{N}$. The samples were then transferred to gelatin and let at $37^{\circ} \mathrm{C}$ until they completely submerged into the medium. Tissues were orientated in gelatin that let solidify at $4^{\circ} \mathrm{C}$ before frizzed by isopentyl at $-80^{\circ} \mathrm{C}$. For wholemount ISH, embryos were washed with PBS- $0.1 \%$ tween 20 after $\mathrm{O} / \mathrm{N}$ fixation, and then dehydrated by $\mathrm{MeOH}$ washes $(50 \% \mathrm{MeOH}, 100 \% \mathrm{MeOH})$ and stored in $100 \% \mathrm{MeOH}$ at $-80^{\circ} \mathrm{C}$.

\section{Immunofluorescence (IF)}

Sections of paraffin-embedded tissue were incubated in xylol for $5 \mathrm{~min}$ (twice) followed by two washes in $100 \% \mathrm{EtOH}$ for $5 \mathrm{~min}$ and subsequently washes in $90 \%, 70 \%, 50 \%$ and $30 \% \mathrm{EtOH}$ for $1 \mathrm{~min}$ each. For antigen retrieval the sections were boiled for $15 \mathrm{~min}$ in $5 \mathrm{mM}$ citrate buffer and endogenous peroxidases were blocked with $3 \% \mathrm{H}_{2} \mathrm{O}_{2}$ in $\mathrm{MQ}$ for $30 \mathrm{~min}$. The tissues were permeabilized with PBT - TX100 and washed with PBS before incubation with the blocking solution ( $2 \%$ bovine serum albumin, $10 \%$ goat serum and $2 \mathrm{mM} \mathrm{MgCl}_{2}$ in PBS) for at least $3 \mathrm{~h}$. For 
the antibodies that the signal was amplified by biotin-streptavidin, sections were additionally blocked with the Biotin-Streptavidin blocking kit (Vector). The primary antibodies (Table5) were diluted in blocking solution and sections were incubated $\mathrm{O} / \mathrm{N}$ at $4^{\circ} \mathrm{C}$. The following day, sections were washed with PBS followed by incubation with the secondary antibody in blocking solution at room temperature (RT) for $1 \mathrm{~h}$. For biotin-streptavidin signal amplification, a secondary biotinconjugated antibody was used followed by a fluorescent-conjugated streptavidin antibody. For tyramide signal amplification (TSA), a secondary antibody coupled to horseradish peroxidase (1h at RT, Dako Cytomation) was used followed by incubation with the substrate (TSA, Perkin Elmer). Sections were washed in PBS before incubation with DAPI (Sigma-Aldrich) for 10 min. Finally, sections were mounted in fluoromount (Southern Biotech). Similar procedure was followed for sections of gelatin-embedded tissue. Sections were left for $15 \mathrm{~min}$ at $37^{\circ} \mathrm{C}$ to dissolve the gelatin and then they were washed with PBS before incubation with the blocking solution. The primary antibody was diluted in blocking solution and let $\mathrm{O} / \mathrm{N}$ at $4^{\circ} \mathrm{C}$. Sections were washed with PBS before incubation with the secondary antibody for $1 \mathrm{~h}$ at RT and washed with PBS before incubation with DAPI. Tyramide or biotin-streptavidin signal amplification was done as paraffin sections.

Table 5. List of antibodies

\begin{tabular}{|l|l|l|l|l|}
\hline Name & From & IF & Amplification & WB \\
\hline Caveolin-1 & BD Transduction & $1 / 200$ & TSA & $1 / 1000$ \\
\hline Caveolin-1 & Cell Signaling Technology & $1 / 200$ & TSA & $1 / 1000$ \\
\hline PTRF (Cavin1) & Atlas antibodies & $1 / 200$ & TSA & $1 / 200$ \\
\hline Caveolin-2 & St John's Laboratory & $1 / 100$ & TSA & - \\
\hline Mdka & (Calinescu et al., 2009b) & $1 / 200$ & TSA & $1 / 250$ \\
\hline GFP & Aves labs & $1 / 100$ & - & - \\
\hline GFP & Clontech Living Colors & $1 / 200$ & - & - \\
\hline MEF-2 & Santa Cruz & $1 / 100$ & Biotin/Streptavidin & - \\
\hline Tropomyosin & DSHB & $1 / 100$ & - & - \\
\hline MF-20 & DSHB & $1 / 20$ & - & - \\
\hline phosho-Smad3 & Abcam & $1 / 200$ & TSA & - \\
\hline Aldh1a2 & Gene Tex & $1 / 200$ & - & - \\
\hline alpha Tubulin & Thermo fisher & - & - & $1 / 5000$ \\
\hline BrdU & BD Transduction & $1 / 30$ & - & - \\
\hline Zn12 & $\begin{array}{l}\text { Zebrafish International Resource } \\
\text { Center (ZIRC) }\end{array}$ & $1 / 20$ & - & - \\
\hline Zns5 & $\begin{array}{l}\text { Zebrafish International Resource } \\
\text { Center (ZIRC) }\end{array}$ & $1 / 20$ & - & - \\
\hline
\end{tabular}




\section{Transmission electron microscopy (TEM)}

Hearts were fixed in 1\% glutaraldehyde/4\% PFA in PBS overnight. Samples were postfixed in $1 \%$ osmium tetroxide for $60 \mathrm{~min}$ and dehydrated through a series of ethanol solutions $(30 \%$, $50 \%, 70 \%, 95 \%, 100 \%$ ) and acetone. After the last dehydration step, samples were incubated in a 1:3, 1:1, 3:1 mixture of DURCUPAN resin and acetone and cured at $60^{\circ} \mathrm{C}$ for $48 \mathrm{~h}$. Ultrathin sections $(50-60 \mathrm{~nm})$ were obtained using a diamond knife (Diatome) in a ultramicrotome (Leica Reichert ultracut S) and collected in 200-mesh copper grids. The sections were counterstained with $2 \%$ uranyl acetate in water from $20 \mathrm{~min}$ followed by a lead citrate solution (Reynolds, 1963). They were examined with a JEOL JEM1010 electron microscope (Tokyo, Japan) equipped with an Orius SC200 digital camera (Gatan Inc).

\section{In situ hybridisation on sections (ISH)}

Paraffin sections were washed twice with xylol and rehydrated by a series of EtOH washes (2x100\%, 90\%, 70\%, 50\%, 30\% $5 \mathrm{~min}$ each) followed by fixation with 4\% PFA for $20 \mathrm{~min}$. After PBS washes the sections were digested with $10 \mu \mathrm{g} / \mathrm{ml}$ proteinase $\mathrm{K}$ for $15 \mathrm{~min}$ at $37^{\circ} \mathrm{C}$ water bath and fixed again with $4 \%$ PFA for 5 min. After fixation they were incubated with $0,07 \mathrm{~N}$ HCL (15min) and with $0,25 \%$ acetic anhydride in $0,1 \mathrm{M}$ triethanolamine solution (10 min). The sections were then incubated at $65^{\circ} \mathrm{C}$ with hybridisation buffer $(50 \%$ formamide, $5 \mathrm{x}$ saline-sodium citrate (SSC) buffer, $0,1 \%$ Tween-20, $50 \mu \mathrm{g} / \mathrm{ml}$ heparin, $5 \mathrm{mg} / \mathrm{ml}$ yeast RNA) for at least $3 \mathrm{~h}$ and then the riboprobe was added in the hybridization buffer and incubated $\mathrm{O} / \mathrm{N} 65^{\circ} \mathrm{C}$. The following day, sections were washed with post-hybridization buffer I ( $50 \%$ formamide, $0,2 \mathrm{x} \mathrm{SSC} \mathrm{pH5,5,} \mathrm{1 \%} \mathrm{SDS)}$ and II ( $50 \%$ formamide, $0,5 \times \mathrm{SSC}$ pH5,5, 5\% SDS) for $2 \times 30 \mathrm{~min}$ each at $65^{\circ} \mathrm{C}$, that was followed by washes in $100 \mathrm{mM}$ maleic acid (MABT). Next, the sections were incubated with blocking solution ( $2 \% \mathrm{FBS}, 1 \%$ blocking reagent - Roche, in $1 \mathrm{x}$ MABT) for $3 \mathrm{~h}$ at RT. The slides then were incubated with the anti-Digoxigenin antibody (Roche) coupled to alkaline phosphatase in blocking solution $\mathrm{O} / \mathrm{N}$. The following day, the sections were washed for $3 \mathrm{~h}$ in MAPT and then in AP buffer $\left(0,1 \mathrm{M} \mathrm{NaCl}, 0,05 \mathrm{M} \mathrm{MgCl}_{2}, 0,1 \mathrm{M}\right.$ Tris-HCL pH9,5, 10\% Tween-20 and 0,002M tetramisole hydrochloride - Sigma Aldrich) 2 x 10 min; followed by incubation in BM-purple (Sigma Aldrich) for the development of the signal. The reaction was stopped by PBS washes and the sections were fixed in 4\% PFA for 15 min followed by dehydration washes via EtOH series. After xylene washes the sections were mounted with Entellan (Merk Millipore) and let dry at RT. 


\section{Fluorescence ISH followed by IF}

The ISH procedure is followed up to the MAPT washes in the second day. Subsequently, the sections are incubated by the anti-Digoxigenin-POD (Sigma-Aldrich) in IM-blocking solution $\mathrm{O} / \mathrm{N}$ at $4^{\circ} \mathrm{C}$. The sections washed in MAPT for $3 \mathrm{~h}$ and are incubated by an antibody coupled to horseradish peroxidase (1h at RT, Dako Cytomation). After PBS washes the primary antibody was added to the slides for $\mathrm{O} / \mathrm{N}$ incubation at $4^{\circ} \mathrm{C}$. The following day, the sections are incubated with the secondary antibody and DAPI similar to the IF protocol.

\section{Whole mount in situ hybridisation (WM-ISH)}

Embryos and fins were stored in $100 \% \mathrm{MeOH}$ at $-80^{\circ} \mathrm{C}$ were rehydrated by $\mathrm{MeOH}$ serial washes $(75 \%, 50 \%, 25 \% \mathrm{MeOH}$ in PBS-Tween $0,1 \%)$ followed by PBS-Tween 0,1\%. The samples were then digested by proteinase $\mathrm{K}$ according to the type of tissue and the stage (fins $20 \mu \mathrm{g} / \mathrm{ml} 20$ min, embryos $10 \mu \mathrm{g} / \mathrm{ml}$ : 2dpf $15 \mathrm{~min}$, 3dpf $20 \mathrm{~min}$ ), washed in PBS-Tween 0,1\% fixed in $4 \%$ PFA for $20 \mathrm{~min}$. After washes with PBS the samples we incubated with the hybridization solution $(50 \%$

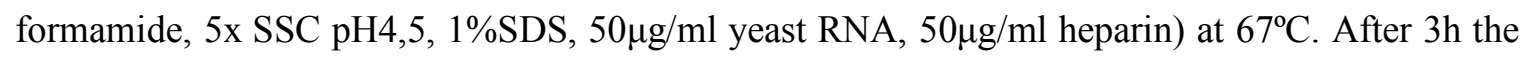
riboprobe was added to the samples with fresh hybridization solution for $\mathrm{O} / \mathrm{N}$ incubation. The samples were washed with saline sodium citrate with $0,1 \%$ Tween 20 (SSCt) as follows 3 x 20 min $50 \% 2 \mathrm{mM} \mathrm{SSCt} / 50 \%$ Formamide, $2 \times 20 \mathrm{~min} 2 \mathrm{mM} \mathrm{SSCt} 2 \mathrm{mM}, 3 \times 30 \mathrm{~min} 0,2 \mathrm{mM} \mathrm{SSCt}$. Next, the sections were incubated with blocking solution ( $2 \%$ FBS, $1 \%$ blocking reagent - Roche, in PBS) for $3 \mathrm{~h}$ at RT and incubated with the anti-digoxigenin antibody coupled to alkaline phosphatase in blocking solution $\mathrm{O} / \mathrm{N}$. The following day the samples were washed with PBS-Tween $0,1 \%$ for $3 \mathrm{~h}$ that proceeded by AP buffer washes for 3 x $5 \mathrm{~min}$. The BM-purple was added to the samples until developing of the colour. The reaction was stopped by PBS washes and the samples were fixed in $4 \%$ PFA O/N and subsequently PBS washes. The tissue or the embryos were mounted on gelatin for imaging.

\section{Histological Stains}

Acid fuchsin orange G-staining (AFOG) is described elsewhere (Poss et al., 2002), and Picro-Sirius red stain performed using standard procedures (Sigma).

\section{RNA extraction and cDNA preparation}

Embryos or hearts were homogenized in Trizol (Invitrogen) using the TissueLyser LT Adapter (Biocompare). To isolate the RNA the Direct-zol RNA MiniPrep kit (Zymo Research) was used and for the cDNA synthesis the SuperScript III First Strand (Invitrogen). 


\section{Quantitative RT-PCR (qPCR)}

Three to five biological replicas (pools of embryos or hearts) with three technical replicas each sample were used for the expression analysis of genes with qPCR (Table 6). The power SYBR Green Master Mix (Applied Biosystems) was used together with the ABI PRISM 7900HT FAST Real-Time PCR System. All measurements were normalized to the elfa or rspm level of expression for the adult heart and for the embryos to the elfa or $18 s$ (McCurley and Callard, 2008). Depending on the experiment, Brown-Forsythe and Welch ANOVA tests or two-tailed unpaired t tests were used for statistical analysis.

Table 6. qPCR primer sequences

\begin{tabular}{|c|c|c|}
\hline Name & Sequence & Reference \\
\hline $18 s$ Fwd & TCGCTAGTTGGCATCGTTTATG & \multirow{4}{*}{$\begin{array}{l}\text { (McCurley and Callard, } \\
\text { 2008) }\end{array}$} \\
\hline $18 s$ Rvs & CGGAGGTTCGAAGACGATCA & \\
\hline elfa Fwd & CAGCTGATCGTTGGAGTCAA & \\
\hline elfa Rvs & TGTATGCGCTGACTTCCTTG & \\
\hline cav1 Fwd & TGGGATGGGGGAATGGAAAC & \multirow{6}{*}{ this thesis } \\
\hline cav1 Rvs & AАCСCTTCACTTCTGCTCACA & \\
\hline cav2 Fwd & GCGTTTATTGCAGGGATTGT & \\
\hline cav2 Rvs & GGATCACTGGCATCACCAC & \\
\hline cav3 Fwd & CAACGAAGATGTCGTGAAGG & \\
\hline cav3 Rvs & GAGACGGTGAAGGTGGTGTAA & \\
\hline cavin $1 b$ Fwd & TCCTGACCACACCATCTACG & \multirow{4}{*}{ (Lim et al., 2017) } \\
\hline cavin $1 b$ Rvs & ATGATCTTCCACCAGCACCA & \\
\hline cavy Fwd & CCTCTGATCAATGGACGTGA & \\
\hline cavy Rvs & AAAACCAGACCCTGGAAACC & \\
\hline mdka Fwd & ACCGTCTCTGTGACCAAACC & \multirow{2}{*}{ (González Rajal, 2012) } \\
\hline mdka Rvs & СТTTCСССТTGCСТTТСТTТ & \\
\hline mdkb Fwd & CAGCATCATATTCACAGCAA & \multirow{4}{*}{ this thesis } \\
\hline$m d k b$ Rvs & AGAGCTATAGAGAACAAACTCC & \\
\hline ptn Fwd & AAGATGCAAGACTCTCAGAA & \\
\hline ptn Rvs & GTTTCGCTCCTTCTTCTTTC & \\
\hline
\end{tabular}

\section{Microscopy and confocal imaging}

Whole mount images of hearts, embryos and fins were taken with a Leica stereoscope (MZFLIII) connected to an Olympus camera DP71. The Olympus BX51 fluorescent microscope 
with a Nikon DP71 camera was used for images of ISH or AFOG staining. IF sections were imaged by the Leica SP8 or the Zeiss LSM 700 confocal microscopes.

\section{Whole mount confocal imaging}

Endogenous fluorescent of whole mount hearts was done as descripted elsewhere (Münch et al., 2017). Briefly, hearts were fixed $\mathrm{O} / \mathrm{N}$ at $2 \%$ PFA and after PBS washes the tissues were immersed in 3\% agarose. The samples then were incubated in CUBIC I solution (Susaki et al., 2014) at $37^{\circ} \mathrm{C}$ for one week. Agarose blocks containing the hearts were mounted to imaging petri dish and approximately $700 \mu \mathrm{m}$ of the injured ventricle was scanned by a 10x objective in the Leica SP8 confocal microscope (z-stack step size $3 \mu \mathrm{m}$ ). For whole mount embryos imaging, the embryos were let in fish water with tricaine $0,16 \%$ on ice and then they were immersed in $3 \%$ agarose horizontally (transverse) or on their lateral side (sagittal). Fins were fixed with 2\% PFA washed in PBS and placed on an imaging petri dish for analysis.

\section{Image analysis}

To analyse the proliferation ratio of the cardiomyocyte, MEF2 positive nuclei were counted in an area $100 \mu \mathrm{m}$ around the injured site using Fiji. BrdU - MEF2 positive cells were also counted and the \% proliferation index was expressed as MEF2 / BrdU-MEF2 ratio. For the proliferative state of the epicardial-derived cells, all GFP-DAPI positive and BrdU- GFP-DAPI cells were counted and presented as above. For TGF-b signalling activation, all psmad3 positive cardiomyocyte ( $100 \mu \mathrm{m}$ of the injury) or GFP positive cells (inside the injured area) were counted and related to the total number of the CM or GFP positive cells. For quantification of the regeneration process, the inured area (fibrotic tissue and collagen) was measured using Fiji and expressed as percentage of the total ventricular area. In the analysis performed on sectioned tissue, at least three sections per samples were used for the measurements. The 3D analysis of the WMheart imaging was carried out by Fiji and IMARIS programmes. The volume of the GFP signal inside the injured area (RFP negative) was also measured and presented in relation to the volume of the injury. Electron microscopy images of the plasma membrane of coronary endothelial cell were taken at 50,000x magnification. Uncoated membrane invaginations of 40-90 nm size and cytosolic vehicles were counted ( $\mathrm{Lim}$ et al., 2017) and expressed as density per $\mu \mathrm{m}^{2}$ of the perinuclear area. Two endothelial cells per three sections of the same heart. The Fiji program was used to calculate the perinuclear area and for caveolae identification.

\section{Statistical analysis}

Sample sizes, statistical test and $\mathrm{P}$ values are specified in the figure legends and performed by the Graph-Prism software. 
Results 


\section{Midkine-a in heart regeneration}

\section{Expression of $m d k a$ in regenerating heart}

We found mdka highly upregulated upon cardiac injury (González Rajal, 2012), so we started by analysing $m d k a$ expression in cryoinjured hearts by in situ hybridization (ISH) (Figure 4A-D', Sup. Figure 1). In control and 12-hours post cryoinjury (hpci) hearts $m d k a$ was not expressed (Sup. Figure 1A-B'). The onset of $m d k a$ expression was one-day post cryoinjury (dpci) in the epicardium, and by 3 dpci was widely expressed in the epicardium (Figure 4A-B'). At 7dpci, the expression became restricted to the multi-layered epicardium that has covered the site of the injury (Figure $\mathrm{C}-\mathrm{C}^{\prime}$ ) while in a later regeneration time point, $14 \mathrm{dpci}, m d k a$ was detected again in the epicardium (Figure 4D-D'). Interestingly, $m d k a$ was detectable up to 130dpci in the epicardium, in the area between the cortical and trabecular myocardium, as well as in the compact layer

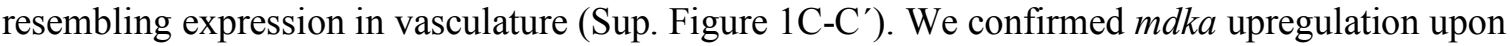
injury by quantitative PCR (qPCR) where the highest was at $3 \mathrm{dpci}$, with a small decrease but still high expression at 7dpci (Figure 4E). To investigate further the epicardial localization of $m d k a$, we performed fluorescence ISH (FISH) in heart sections from the $T g(w t 1 b: G F P)$ line that expresses GFP in $w t 1 b^{+}$epicardial cells (González-Rosa et al., 2012) followed by immunostaining for GFP and Aldh1a2, an enzyme involved in synthesis of RA expressed in epicardial and endocardial cells upon injury (Kikuchi et al., 2011b) (Figure 4F-G'"'). Mdka had overlapping expression with GFP (arrowheads) and in a broader extent with Aldh1a2 (arrows). Overall, mdka was highly upregulated upon injury and detected mainly in the activated epicardium, that by $7 \mathrm{dpci}$, consists of a heterogenous cell population including macrophages and fibroblasts (González-Rosa et al 2012, K. Kikuchi et al 2011, Kazu Kikuchi et al 2011, Sánchez-Iranzo et al 2018, Sanz-Morejón et al 2019).

\section{Generation of $m d k a$ knock-out zebrafish}

Mdka is not expressed in the heart neither during development nor in intact adult hearts, and morpholino experiments do not cause a heart phenotype (Winkler et al., 2003). Additionally, $m d k a$ is activated after injury of different organs including retina, neural tube, fin (González Rajal, 2012; Gramage et al., 2015; King and Yin, 2016; Rabinowitz et al., 2017) and heart. We reasoned that loss of $m d k a$ may have a significant effect in heart regeneration, since it is not expressed in control adult hearts, but is highly upregulated after injury. Thus, we generated $m d k a$ knock-out (KO) zebrafish by CRISPR/Cas9 technology (Figure 5). The $m d k a$ locus is composed of five exons, and we chose to target the fourth exon, that corresponds to the N-terminal of the protein (Figure $5 \mathrm{~A})$. Gene editing efficiency was high and resulted in equal number of deletions or insertions (Figure 5A). The majority of the predicted Mdka mutants had a premature stop codon, due to frame shift of the open reading frame (ORF) (Figure 5B). To select the appropriate allele, we tested embryos from 

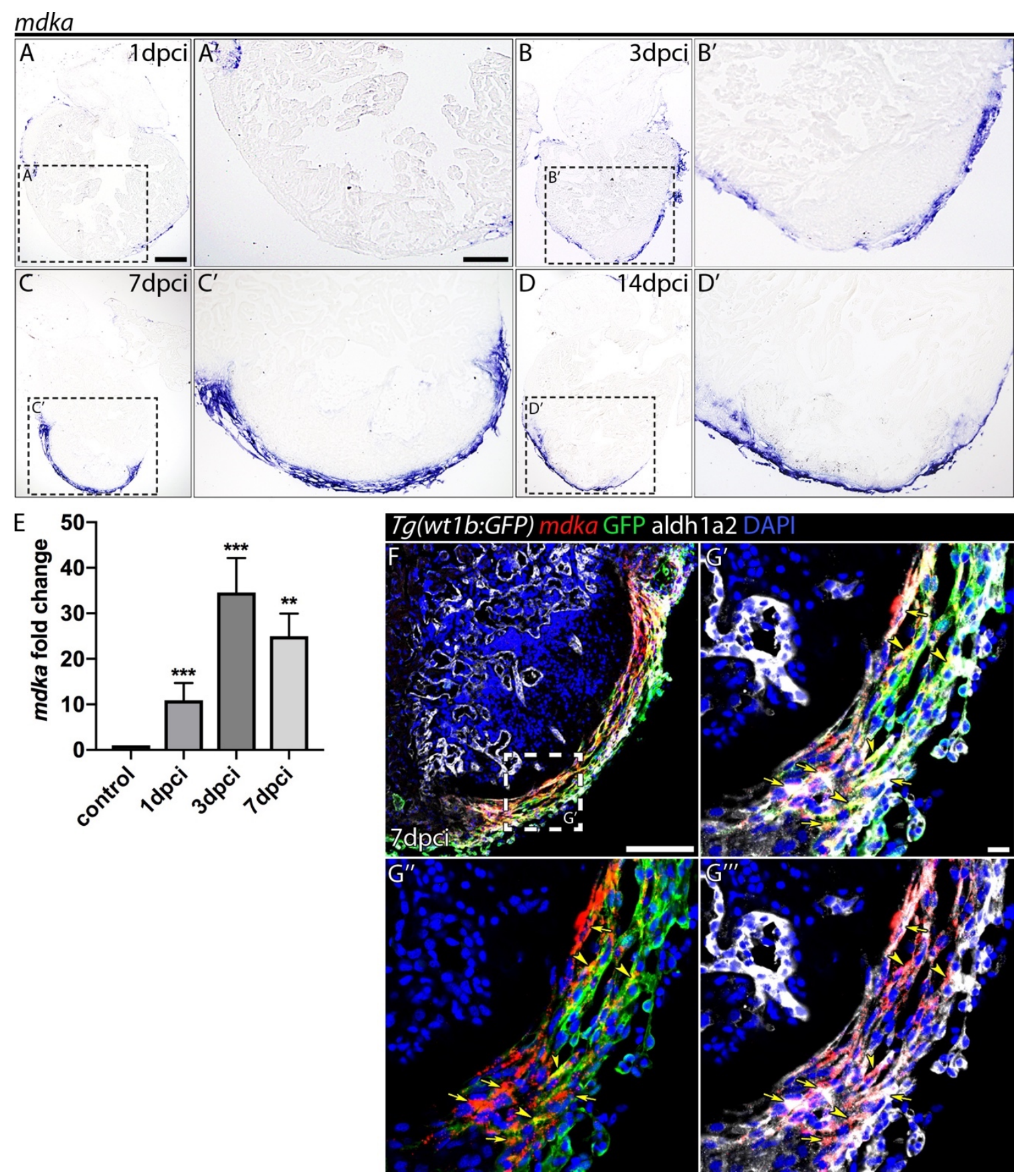

Figure 4. $m d k a$ expression upon injury

(A-D') ISH of $m d k a$ in cryoinjured hearts $1 \mathrm{dpci}\left(\mathrm{A}-\mathrm{A}^{\prime}\right), 3 \mathrm{dpci}\left(\mathrm{B}-\mathrm{B}^{\prime}\right), 7 \mathrm{dpci}\left(\mathrm{C}^{-} \mathrm{C}^{\prime}\right)$ and $14 \mathrm{dpci}$ (D$\left.\mathrm{D}^{\prime}\right)$. Scale bars $100 \mu \mathrm{m}$. (E) qPCR analysis of $m d k a$ in regenerating hearts. Mean \pm S.D., BrownForsythe and Welch ANOVA tests, ${ }^{* *} \mathrm{P}<0.01, * * * \mathrm{P}<0.001$. (F-F"') FISH for mdka followed by immunolabelling of $7 \mathrm{dpci} \operatorname{Tg}(w t 1 b$ : GFP) hearts for GFP and Aldh1a2. Arrowheads indicate $m d k a-$ GFP overlap and arrows $m d k a$-Aldh1a2 overlap. Scale bar F $100 \mu m, F^{\prime}-F^{\prime \prime \prime} 10 \mu m$.

founder fish carrying different genetic mutation by whole-mount in situ hybridization (WM-ISH) and we found that all examined embryos had a significant drop in $m d k a$ expression (Figure 5C). To continue and narrow down the candidates, we performed western blot (WB) analysis to examine 
the protein expression (Figure 5D). Embryos harbouring homozygous genetic mutations lost completely Mdka, in contrast to the heterozygous (HET) and wild-type (WT) zebrafish. Next, we investigated if in the absence of $m d k a$, midkine- $b$ ( $m d k b$ ) and pleiotrophin (ptn) were deregulated. We tested this possibility by qPCR and WM-ISH (Figure 6). Mdkb did not change as detected by qPCR and WM-ISH, that revealed strong $m d k b$ expression. In contrast, ptn although by qPCR was not detected any significant difference, by WM-ISH we observed a clear expression reduction in the forebrain of all tested mutants (Figure 6B, arrowheads). Nevertheless, all embryos carrying different mutant alleles presented no phenotype and were fertile. Overall, all examined genetic mutations resulted in $m d k a$ knockout, since lost Mdka protein expression, and additionally they had the same effect on $m d k b$ and $p t n$. Hence, we selected G33fsX53, hereafter $m d k a^{-/}$, for further studies. First, we wanted to confirm that Mdka was lost also in adult heart, therefore, control or cryoinjured hearts from WT, homozygous and heterozygous animals were analysed by WB (Figure 7A). Mdka was not detectable in control hearts from all three genotypes, whereas it was expressed in 7dpci WT and heterozygous hearts. In contrast, Mdka was absent in hearts from homozygous mutant animals. Immunofluorescences staining also confirmed that Mdka was lost (Figure 7B-C). Furthermore, we examined $m d k a^{-/-}$injured hearts for $m d k b$ and $p t n$ expression (Figure 7D-G). In WT hearts, neither $m d k b$ nor $p t n$ were expressed upon injury, similarly to $m d k a^{-/}$hearts. Thus, the genes related to $m d k a$ were not upregulated in response to $m d k a$ deletion in adult hearts after injury.
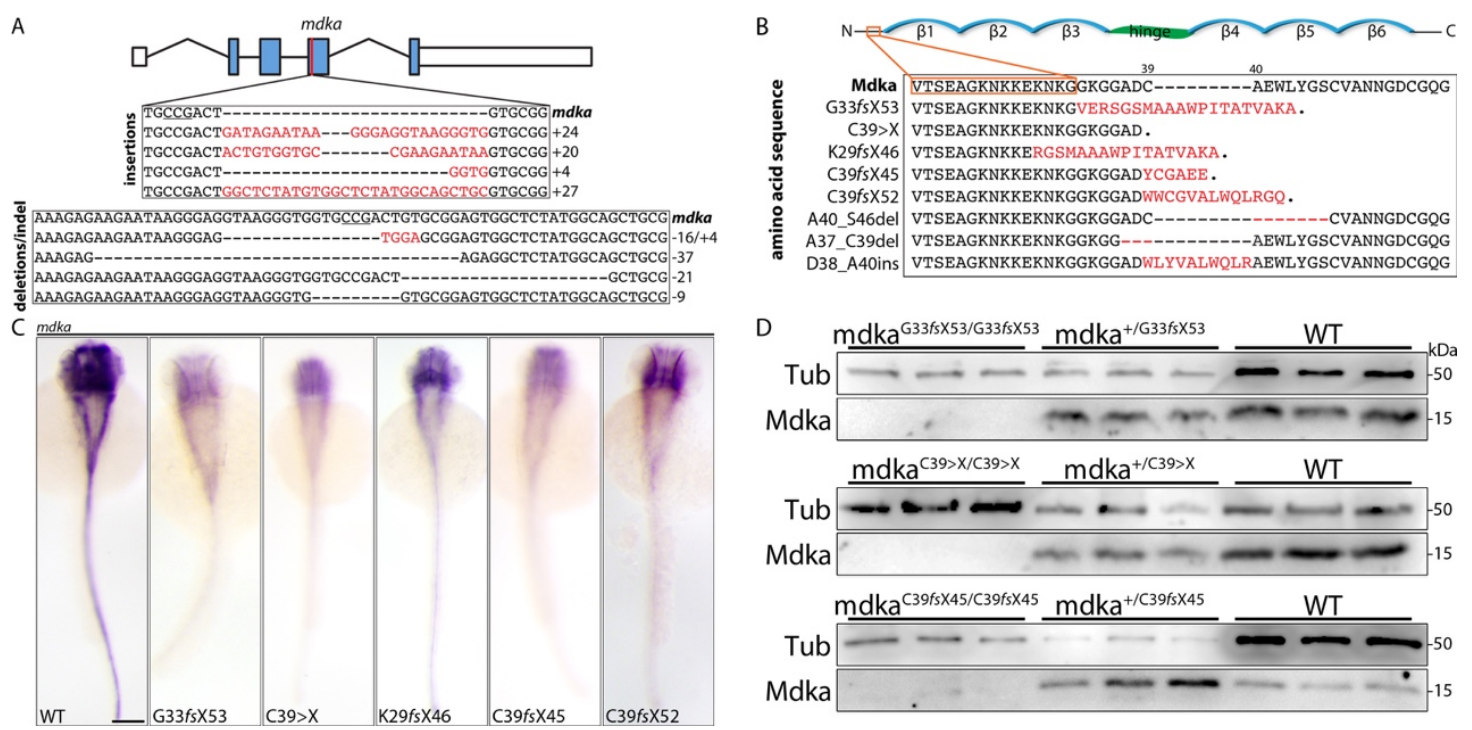

Figure 5. Generation of the mdka-KO zebrafish

(A) Drawing of $m d k a$ locus and DNA mutations introduced by CRISPR/Cas9. Red line $=$ target site. Red letters indicate insertions. (B) Mdka domain organization and predicted mutations in the protein. Red letters denote novel amino acids (a.a.), red hyphen deletions. $\beta=$ beta-strands and in green the conserved hinge domain connecting $\mathrm{N}$-, and C-terminus halves. (C) WM-ISH of twodays post fertilization (dpf) embryos carrying different mutation. Scale bar 200 $\mu \mathrm{m}$. (D) WB analysis for Mdka in WT, heterozygous and homozygous embryos. Tub $=$ Tubulin, $\mathrm{kDa}=$ kilodalton. 


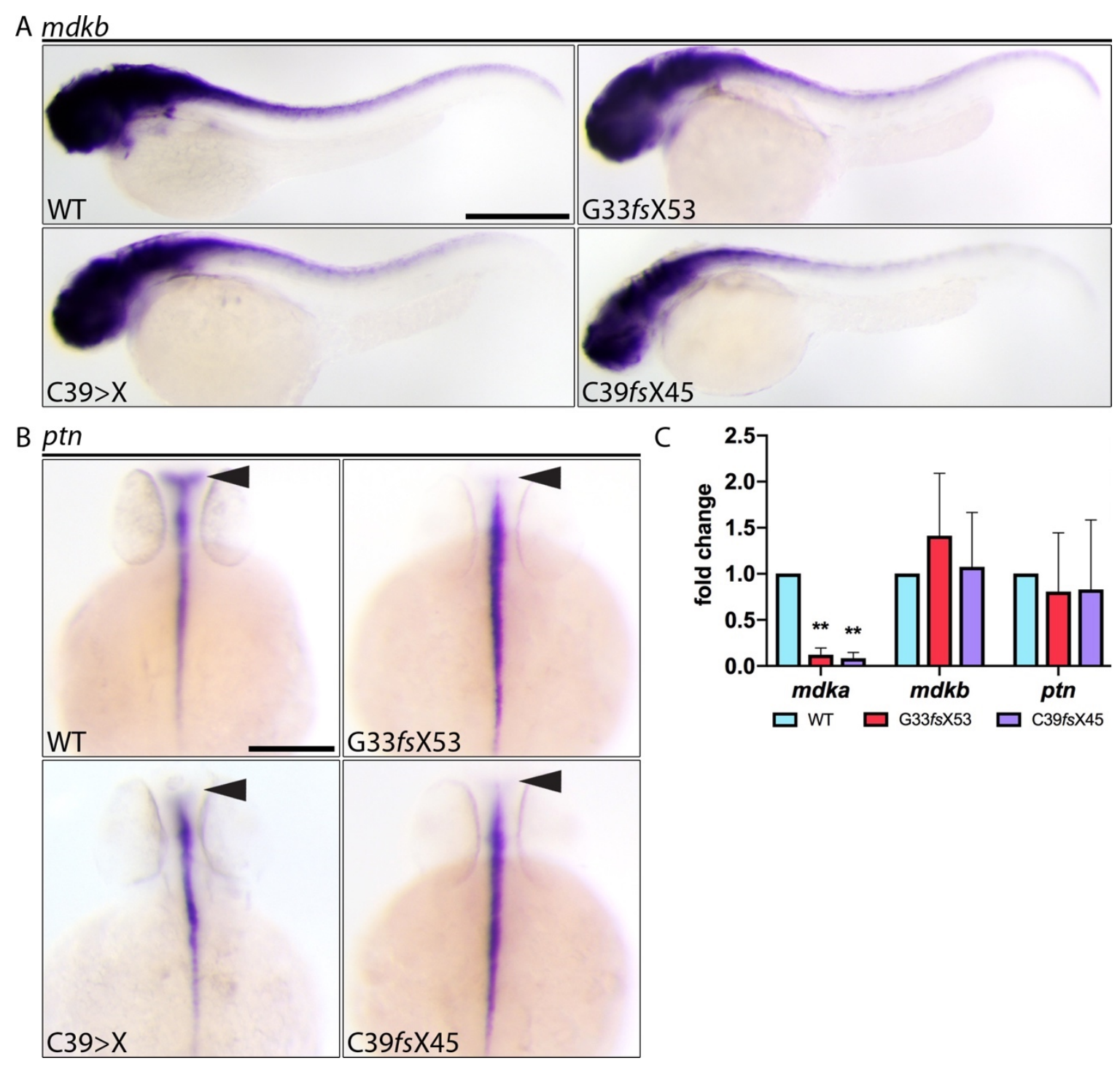

Figure 6. Analysis of $m d k b$ and $p t n$ in mdka mutant embryos

(A) $m d k b$ or (B) $p t n$ WM-ISH in 2dpf $m d k a$ mutant embryos. Scale bars, A $50 \mu \mathrm{m}$ and B $200 \mu \mathrm{m}$.

(C) qPCR of $m d k a, m d k b$ and ptn from selected $m d k a$ mutant embryos. t-test, ${ }^{* *} \mathrm{P}<0.01$, Mean \pm S.D. 

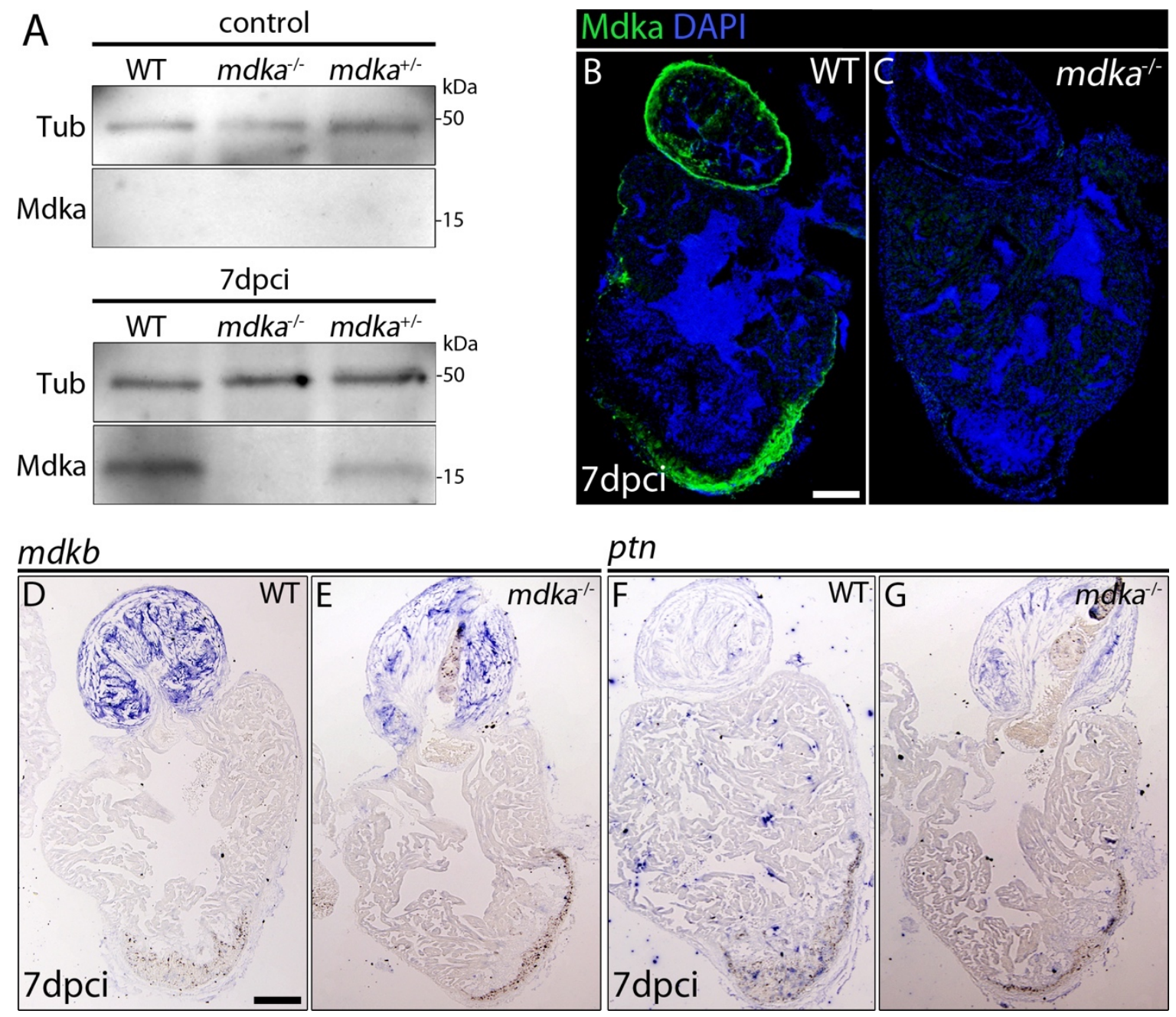

Figure 7. Expression analysis in $\boldsymbol{m d k a ^ { - / }}$ adults heart

(A) Mdka WB analysis in WT and $m d k a^{-/}$, control or 7 dpci hearts. Tubulin (Tub) served as loading control. (B-C) Immunofluorescent staining of Mdka in 7dpci WT or $m d k a^{-/-}$hearts. (D-G) ISH of $m d k b(\mathrm{D}-\mathrm{E})$ and $p t n(\mathrm{~F}-\mathrm{G})$ in $7 \mathrm{dpci}$ WT or $m d k a^{-/-}$hearts. Scale bars $100 \mu \mathrm{m}$. 


\section{mdka-KO hearts fail to regenerate}

We next examined if $m d k a$ inactivation affects heart regeneration. Therefore, we cryoinjured hearts from WT, KO and HET animals and allowed them to regenerate for 90-days. The hearts were harvested and processed for Acid Fuchsin Orange-G (AFOG) staining that labels collagen, fibrin and healthy tissue (Figure 8). The analysis revealed that $m d k a-K O$ hearts had a significant larger injury than the WT and HET siblings (Figure 8D). Additionally, the injured area was characterized by increased collagen deposition in contrast to WT and HET (Figure 8E). These observations indicate that $m d k a$ deletion impairs heart regeneration.

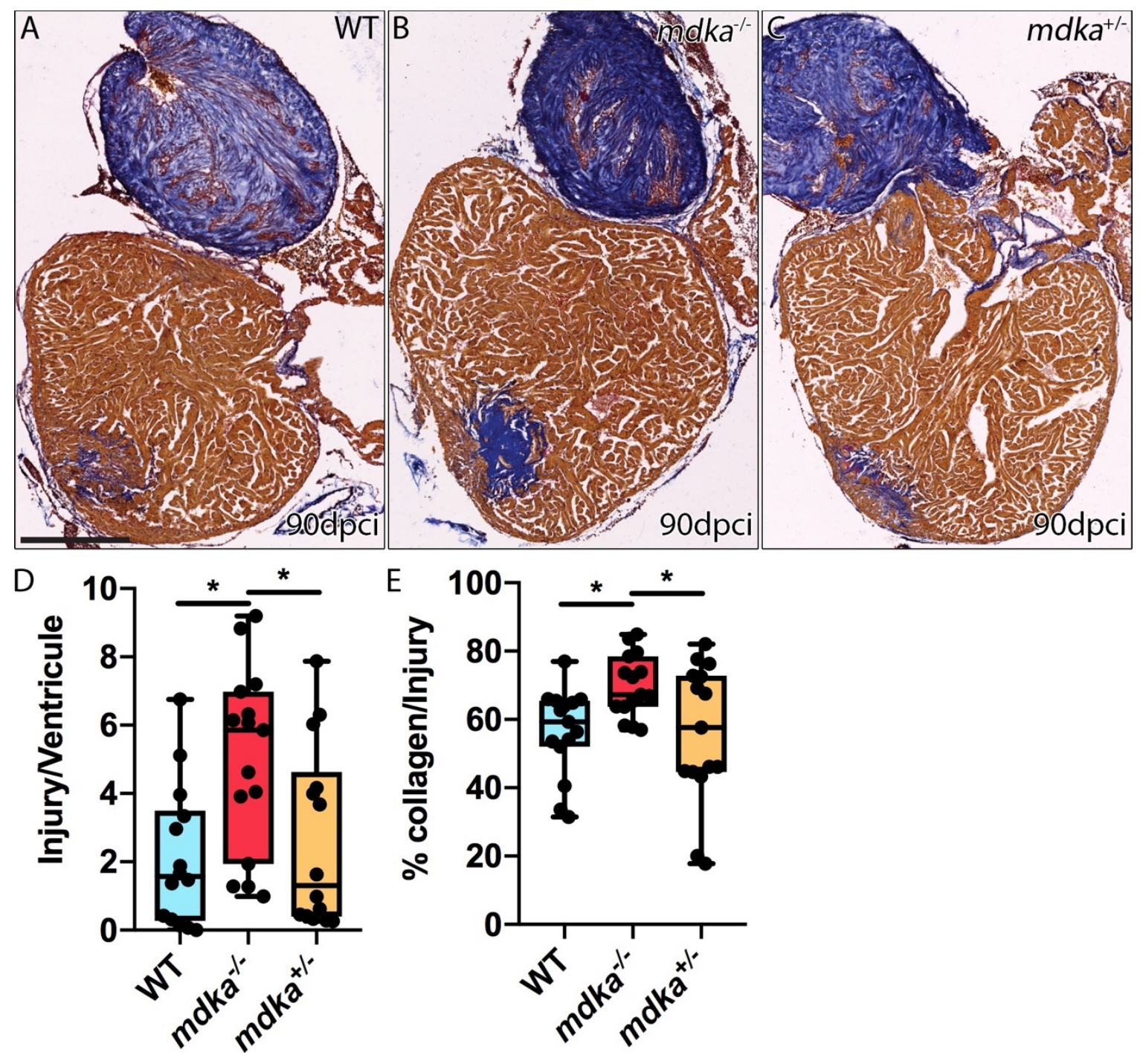

Figure 8. Loss of Mdka leads to arrest of heart regeneration

(A-C) AFOG staining of 90dpci WT, $m d k a^{-/}$and $m d k a^{+-}$. Collagen in blue, fibrin in red, healthy myocardium in brown. Scale bar $250 \mu \mathrm{m}$. (D) Quantification of the injury size as percentage of the damaged tissue (collagen and fibrin) to the ventricular area. $\mathrm{n}_{\mathrm{WT}}=14, \mathrm{n}_{\mathrm{KO}}=15, \mathrm{n}_{\mathrm{HET}}=14$. Ordinary one-way anova. (E) Percentage of the collagen in the injury. $\mathrm{n}_{\mathrm{WT}}=\mathrm{n}_{\mathrm{KO}}=\mathrm{n}_{\mathrm{HET}}=15$. Ordinary oneway anova. 


\section{Cell proliferation analysis in $\mathrm{mdka}^{-/-}$regenerating hearts}

To analyse further the defect in heart regeneration of $m \mathrm{dka}^{-/-}$animals, we examined the proliferation status of the different cell types involved. We started with the epicardial cell $7 \mathrm{dpci}$, in which $m d k a$ had a strong expression. For that, we used the $\operatorname{Tg}(w t 1 b$ :GFP) line and quantified the proliferation rate of the epicardial cells labelled by GFP and Aldh1a2 (Figure 9A-C). Interestingly, the analysis showed that the proliferation of the epicardial cells was not affected by the loss of $m d k a$ (Figure 9C). Next, we focused on the proliferation rate of cardiomyocytes (CM) adjacent to the injury (Figure 9D-F). We labelled 7dpci hearts for BrdU and MEF2 and quantified the $\mathrm{BrdU}^{+} \mathrm{CM}$ in relation to their total number. However, CM proliferation in the KO hearts was similar to the WT (Figure 9F). Overall, loss of Mdka did not affect neither CM nor epicardial cells proliferation.
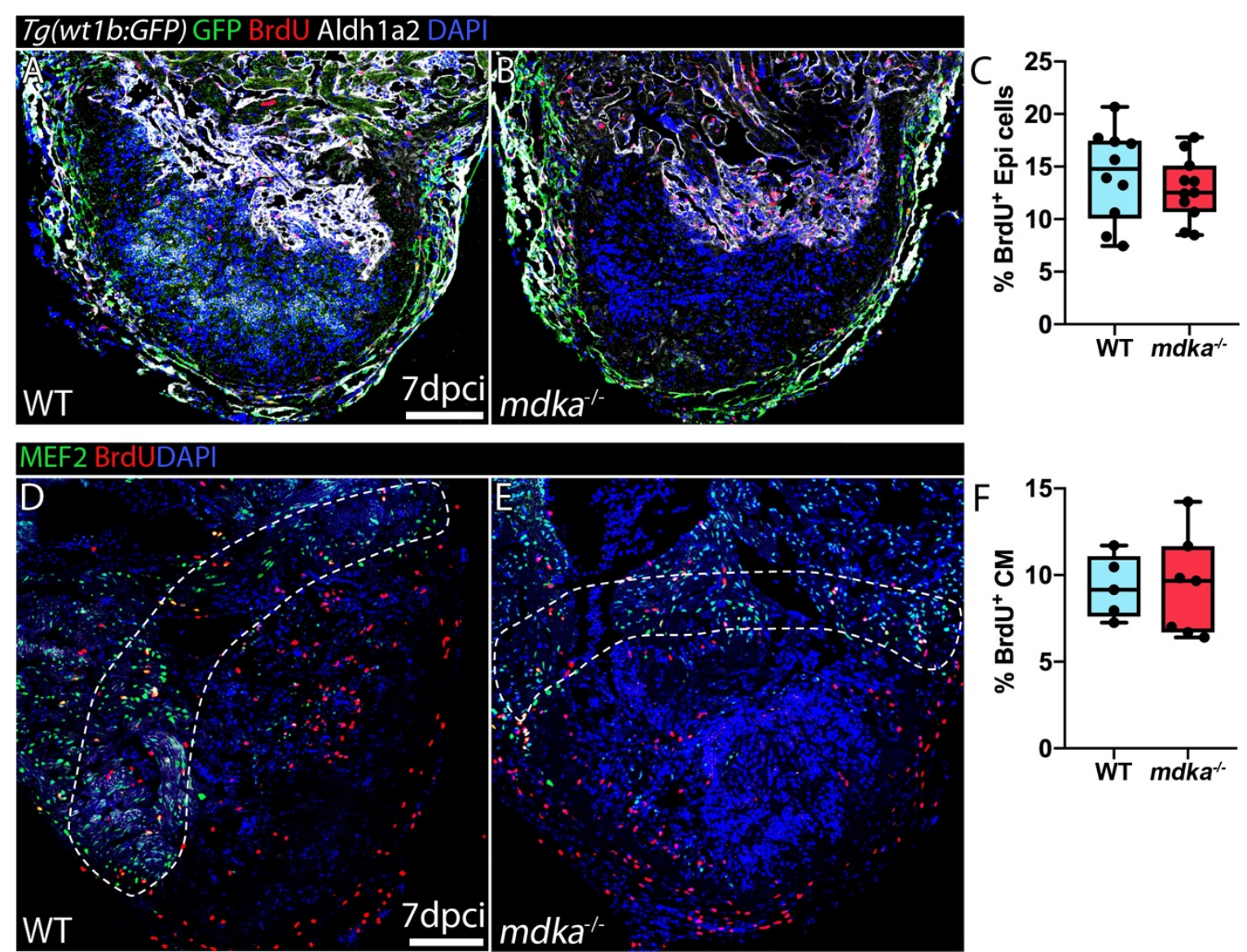

Figure 9. Epicardial cell and CM proliferation in $m d k a^{-/-}$hearts

(A-B) Immunostaining of 7dpci WT (A) or $m d k a^{-/-}$(B) $T g(w t 1 b$ : GFP) hearts for BrdU, GFP and Aldh1a2. Scale bar $100 \mu \mathrm{m}$. (C) Quantification of the BrdU ${ }^{+}$epicardial cells. t-test, $\mathrm{n}_{\mathrm{WT}}=10, \mathrm{n}_{\mathrm{KO}}=$ 11. (D-E) Staining of BrdU and MEF2 in $7 \mathrm{dpci}$ WT and $m d k a^{-/-}$hearts. Scale bar $100 \mu \mathrm{m}$. (F) Percentage of the $\mathrm{BrdU}^{+} / \mathrm{MEF} 2^{+}$cardiomyocytes. t-test, $\mathrm{n}_{\mathrm{WT}}=6, \mathrm{n}_{\mathrm{KO}}=7$. 


\section{Analysis of $m d k a$ epigenetic regulation}

\section{The epigenetic landscape of $m d k a$ locus in development and heart regeneration}

Mdka is a small cytokine that has a strong expression during development (Winkler et al., 2003) and as we showed here, during heart regeneration. In embryos, $m d k a$ is expressed in neural tube and in the brain, whereas in the adult heart is expressed only after injury, mainly in epicardial cell marked by Wt1b and/or Aldh1a2. To investigate such a dissimilar expression, we focused on the epigenetic regulation of $m d k a$, since cis-regulatory elements have been shown to regulate cellspecific spatio-temporal expression (Bogdanović et al., 2012; Kang et al., 2016). Additionally, such an analysis may identify the minimum genomic region that can recapitulate $m d k a$ endogenous expression and can be used as a driver for transgenic lines. To address the transcriptional orchestration of regulatory elements, we took advantage of available sequencing data from chromatin immunoprecipitation (ChIP) from different zebrafish developmental stages and from regenerating hearts 14-days post CM genetic ablation, as well as of sequencing data from assay for transposase-accessible chromatin (ATAC) from embryonic endothelial cells (flila $a^{+}$or flila) (Bogdanović et al., 2012; Goldman et al., 2017; Quillien et al., 2017). We annotated these data to the UCSC genome browser so that we could have a comprehensive view of the epigenetic marks per locus (Figure 10). The H3K4me3 methylation marks (green) denote the transcriptional active promoter, the $\mathrm{H} 3 \mathrm{~K} 4 \mathrm{me}$ (blue) regions with enhancer activity and the H3K27ac acetylation marks (magenta) the transcriptional initiation (Figure 10A) (Dorighi et al., 2017; Wang et al., 2001; Zhang et al., 2013). ChIP-seq data reveal that $m d k a$ promoter (mdka) is flanking the first exon, and in the first long intron, $\mathrm{H} 3 \mathrm{~K} 4 \mathrm{me} 1$ and $\mathrm{H} 3 \mathrm{~K} 27 \mathrm{ac}$ enriched regions uncover three active enhancers, enhancer1, enhancer2 and enhancer3 (enha1, enha2 and enha3) refer to them as enh. Additionally, 5 ' of the gene there are two more enhancers, enhancer4 (enha4) and enhancer5 (enha5), that are located 3 kilobases $(\mathrm{kb})$ and 700 base pair (bp) upstream of the promoter, respectively. ATAC-seq data from $\mathrm{GFP}^{+}$and $\mathrm{GFP}^{-}$cells from 24hpf $T g(f l i l a: G F P$ ) embryos match the ChIP-seq data, since the detected open chromatin regions correspond to developmental methylation or acetylation marks in the promoter and the enhancers. The intensity of the peaks differs between the $\mathrm{GFP}^{+}$and $\mathrm{GFP}^{-}$ cells, for which, the GFP' peaks score higher than the $\mathrm{GFP}^{+}$, suggesting that $m d k a$ epigenetically is induced in not endothelial cells during development. In the regenerating hearts, we were able to map only the genomic coordinates of acetylation marks. In control hearts and 14dpi, the epigenetically active regions are similar to the developmental ones. Interestingly, a region $3^{\prime}$ of the enh seems to be marked only during regeneration with a moderate activation during development. That region appears to be a regeneration-related enhancer named Reg. Similar epigenetic modifications were also observed 48hpf (Sup. Figure 2). We cloned the above regulatory regions into the ZED plasmid (Bessa et al., 2009) that contains the gata2 promoter upstream of GFP and expresses RFP under the Xenopus Cardiac Actin promoter (X.Card.Actin) in all muscles for 
transgenesis control, or into plasmids containing different combinations of $m d k a$ regulatory elements using the endogenous $m d k a$ promoter (Figure 10B). The plasmids were injected into onecells stage zebrafish embryos and the resulting transgenic animals were selected based on their GFP and RFP expression (Figure 10C). Overall, different combinations of $m d k a$ regulatory elements were tested, but we focused on the following transgenic animals: $T g$ (FRT-Xla.Actc1:DsRed-GABFRT,LOXP-mdka-LOXP-gata2a:EGFP-5HS4) referred to as $T g$ (mdka:GFP), $\operatorname{Tg}(F R T$ Xla.Actcl:DsRed-GAB-FRT,LOXP- $\quad+2 m d k a \quad-L O X P$-gata2a:EGFP-5HS4) referred to as $\operatorname{Tg}$ (enh:GFP), Tg(FRT-Xla.Actc1:DsRed-GAB-FRT,LOXP- $\quad$-4.8mdka -LOXP-gata2a:EGFP5HS4) referred to as $T g(e n h a 4: G F P), T g(F R T-X l a . A c t c 1: D s R e d-G A B-F R T, L O X P-+5.8 m d k a-$ LOXP-gata2a:EGFP-5HS4) referred to as $T g(\operatorname{Reg}: G F P), T g(+2 m d k a-m d k a: e G F P)$ referred to as $\operatorname{Tg}(e n h-m d k a: G F P)$, and $T g(-2.5 m d k a-m d k a: e G F P)$ referred to as $T g(e n h a 5: G F P)$. Embryos with fluorescence expression were raised to adulthood, outcrossed to WT and the progeny embryos were studied.
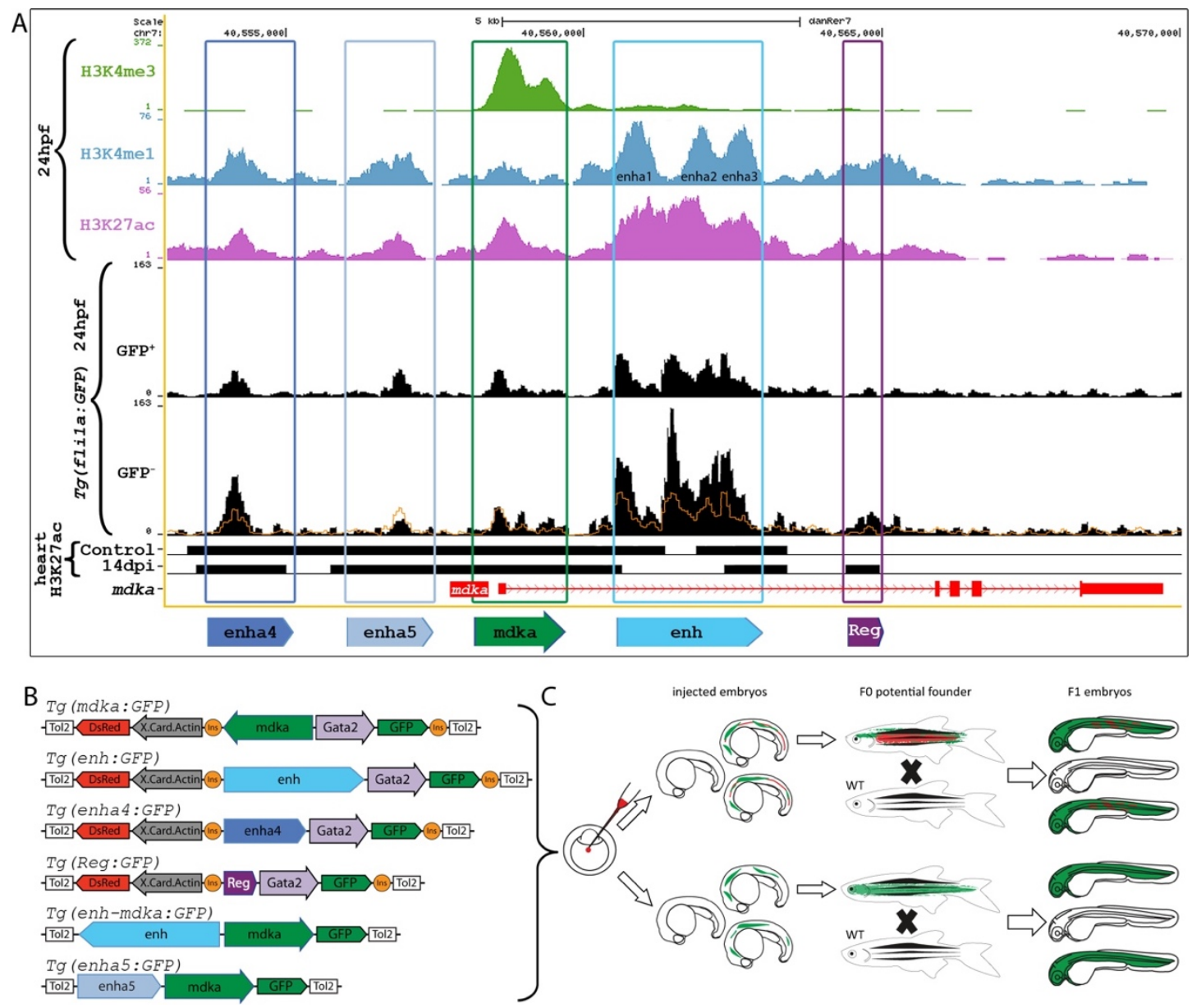

Figure 10. $m d k a$ locus and epigenetic marks in development and heart regeneration

(A) Snapshot of the UCSC genome browser with tracks showing methylation (H3K4me3/1) and acetylation (H3K27ac) marks from 24hpf embryos (peaks) and from control or 14dpi hearts (black boxes); and peaks of ATAC-seq revealing the accessible genomic regions in $\mathrm{GFP}^{+}$and $\mathrm{GFP}^{-}$cells 
from 24hpf $T g(f l i l a: G F P)$ embryos. $m d k a$ in red. The orange line in the $\mathrm{GFP}^{-}$track is the outline of the $\mathrm{GFP}^{+}$peaks to illustrate the difference in intensity. All cloned regions in coloured boxes and the corresponding genomic regions in the associated outlines. (B) Organization of all injected plasmids with their names. X.Card.Actin $=$ Xenopus cardiac actin promoter. ins $=$ insulator. $(\mathrm{C})$ Pipeline of the selection procedure.

\section{Analysis of $T g(m d k a: G F P)$ and $T g(e n h: G F P)$ transgenic lines}

We started the analysis with the promoter of $m d k a$ that in embryos induced the expression of GFP in a specific pattern (Figure 11). GFP was detected in the neural tube of the developing larvae and to some extent in the brain (Figure 11A, B). We traced the GFP expression in the trunk of the embryo from the lateral side to the anteroposterior axis (Figure 11C). GFP was detected in the lateral line of the embryo (Figure 11D, arrowheads), a mechanosensor system comprising neuromasts (hair cells, supporting and mantle cells) that detects motion of the environment in fish [reviewed in (Dambly-Chaudière et al., 2003)], and in neuromasts of the lateral line (Figure 11D, asterisk). Towards the anteroposterior axis, we detected GFP in motor neurons bodies, and axons expanding ventrally (Figure 11E, F brackets and arrowheads, respectively) (Zelenchuk and Brusés, 2011). A pattern that we have not observed by $m d k a$ WM-ISH. Finally, strong GFP expression was also detected in the neural tube (Figure 11G-I, parentheses). We then focused on the adults, and examined the expression after fin amputation and heart cryoinjury (Figure 12). Three-days post amputation (dpa), $m d k a$ is expressed in the blastema - a tissue formed after injury containing undifferentiated cells that is highly proliferative (Nechiporuk and Keating, 2002) - of the regenerating fin (González Rajal, 2012). However, GFP was not induced in the regenerating fin, similarly to the WT negative control and in contrast to the $T g$ (flila:GFP) positive control (Figure 12A-C). Likewise, the $\operatorname{Tg}(m d k a: G F P) 7 \mathrm{dpci}$ hearts did not expressed GFP as the $\operatorname{Tg}(w t 1 b: G F P)$ hearts (Figure 12D-G). Given that our initial interest was in heart regeneration and since the $\operatorname{Tg}(m d k a: G F P)$ line did not expressed GFP upon injury, we examined our next transgenic line. For the $T g(e n h: G F P)$ line we used the intronic enhancers of $m d k a$ that were marked in the epigenetic assays. The GFP expression in embryos was strong and similar to the $\operatorname{Tg}(m d k a: G F P)$ in the neural tube (Figure 13A, B brackets). However, $\operatorname{Tg}($ enh:GFP) expressed GFP in the brain that recapitulated the endogenous $m d k a$ expression when compared to WM-ISH (Figure 13C, D). Additionally, $m d k a$ was expressed also in the pectoral fins, but GFP was weak in transgenic embryos (Figure 13C, D arrowheads). Notably, we did not detect GFP expression in the lateral line or in the neural axons. We further tested the $\operatorname{Tg}(e n h: G F P)$ animals upon injury. However, GFP was not detected neither in amputated fins (Figure 13E, F), nor in regenerating hearts (Figure 13G-H). Overall, we found that $m d k a$ promoter or enh in combination with the gata2 promoter, were able to drive GFP expression in embryos in a similar pattern to $m d k a$ endogenous expression. In contrast, injured fins or hearts were characterized by the lack of GFP signal. 


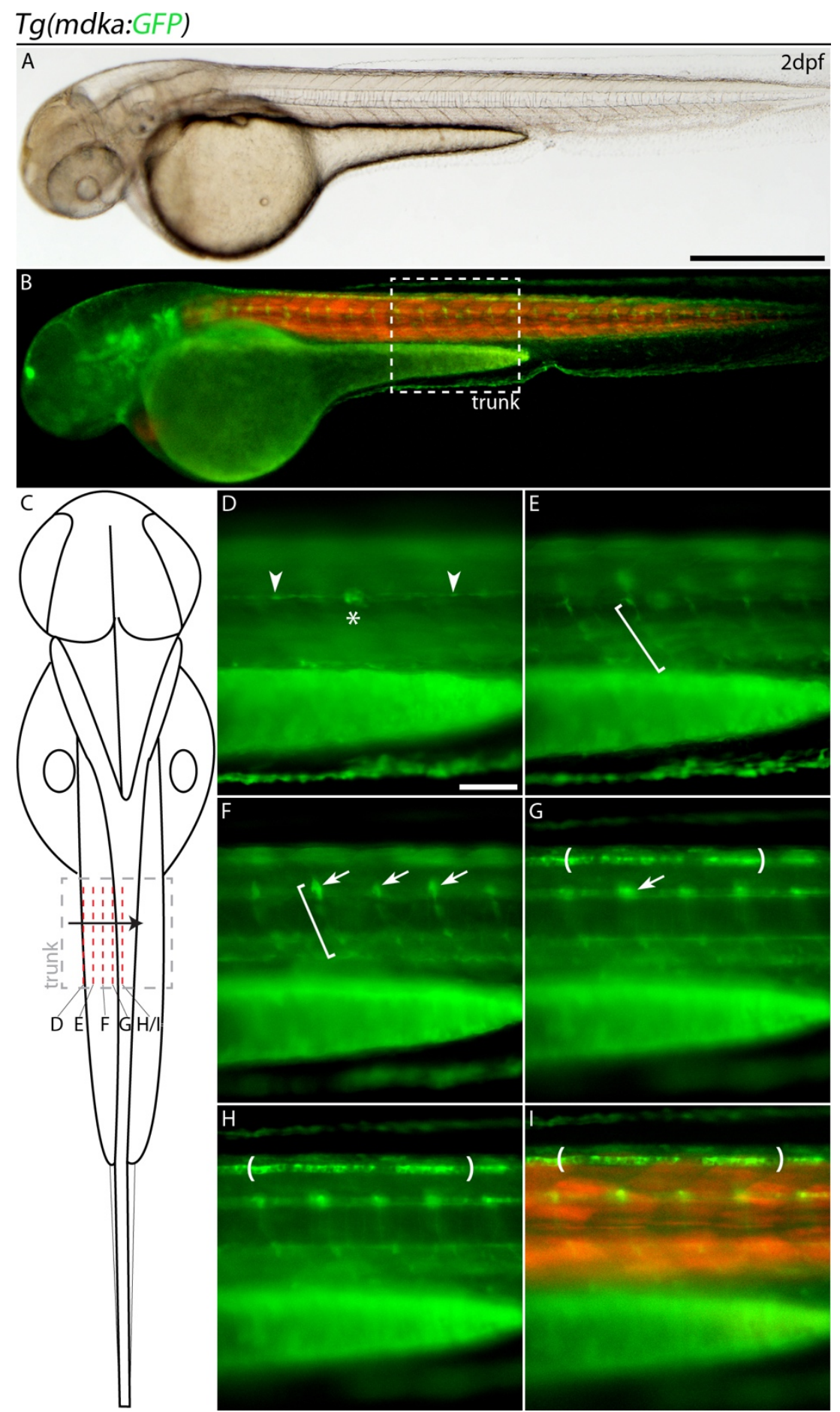

Figure 11. $\mathrm{Tg}(\mathrm{mdka}: G F P)$ expression in embryos

(A, B) $\operatorname{Tg}(m d k a: G F P) 2$ dpf embryo expressing GFP under the promoter of $m d k a$ and RFP under the Xenopus cardiac actin promoter. (C) Dorsal view illustration of a zebrafish embryo indicating the relative position of the D-I images (D-I) Consecutive images of the embryo's trunk from the lateral side towards the neural tube (dashed boxes in B). (D-F) Arrowheads donate GFP expression in the lateral line, asterisk in the neuromasts and brackets indicate the neural axons. (G-I) Parentheses indicate the GFP expression in the neural tube and arrows in the bodies of motor neurons. Scale bars, $500 \mu \mathrm{m}$ in A, B and $100 \mu \mathrm{m}$ in D-I. 

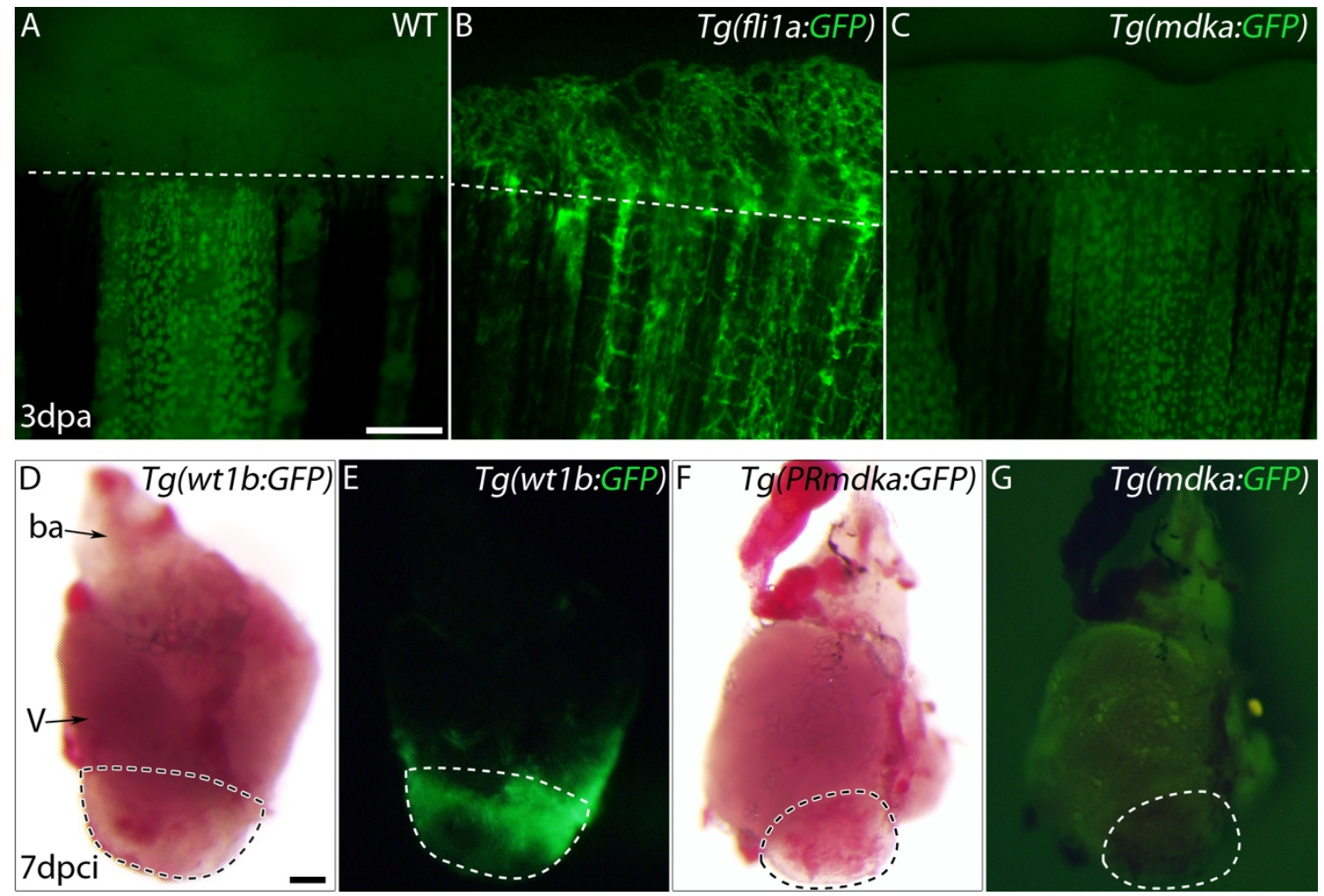

Figure 12. $\operatorname{Tg}(m d k a: G F P)$ in adult tissues upon injury

(A-C) Amputated fins from WT (A), $\operatorname{Tg}(f l i 1 a: G F P)(B)$ and $\operatorname{Tg}(m d k a: G F P)(\mathrm{C})$ examined under a fluorescence microscope. Dashed line = amputation plane. (D-G) $7 \mathrm{dpci} \operatorname{Tg}(w t 1 \mathrm{~b}: G F P)$ and $T g(m d k a: G F P)$ hearts examined for GFP expression. Injured site $=$ dashed area. $\mathrm{V}=$ ventricle, ba $=$ bulbous arteriosus. Scale bars, $100 \mu \mathrm{m}$ in A-C, $200 \mu \mathrm{m}$ in D-G. 

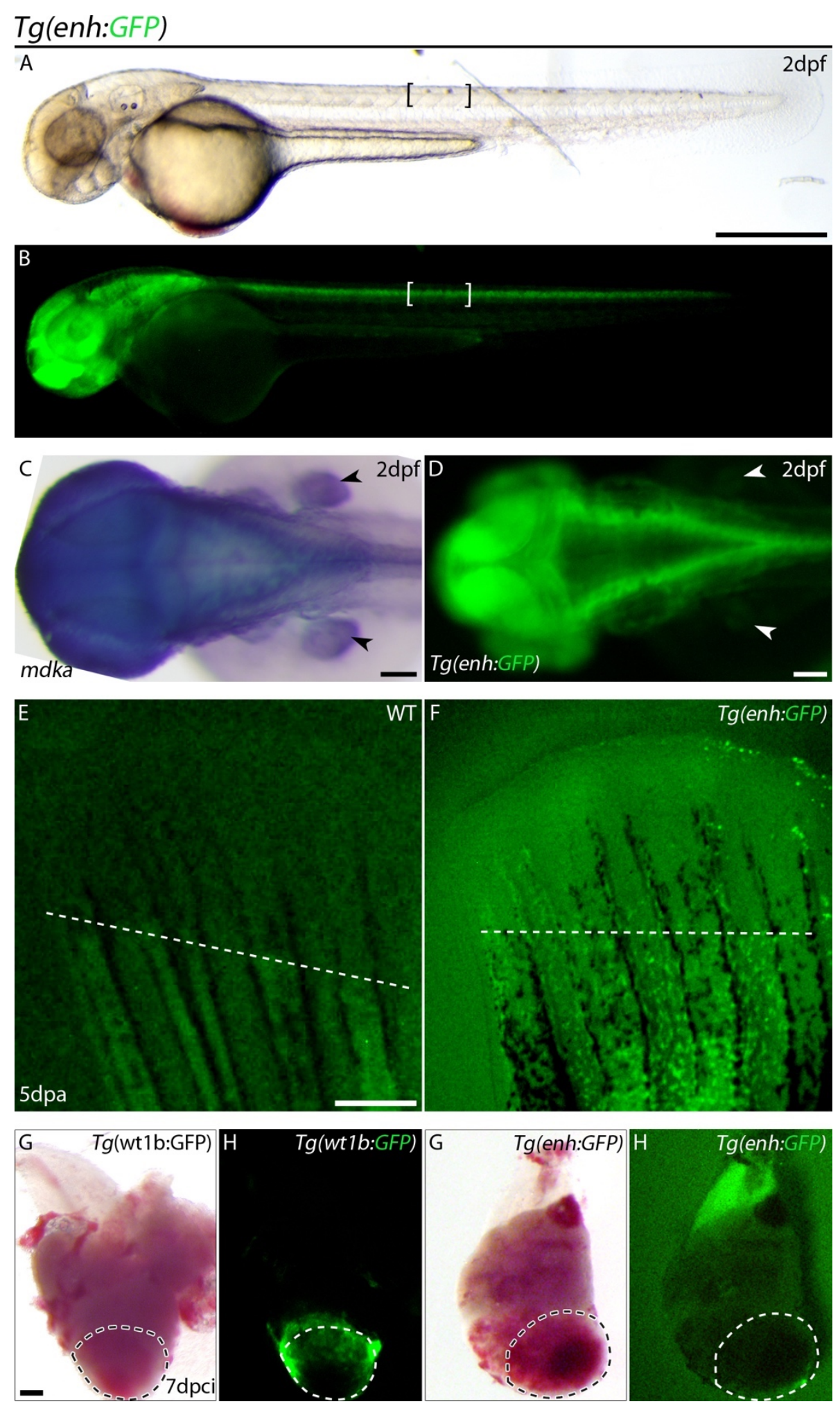

Figure 13. $\mathrm{Tg}(\mathrm{enh}: \mathrm{GFP})$ expression in embryos and adults

(A, B) $\operatorname{Tg}($ enh:GFP) embryos 2 dpf expressing GFP in the neural tube (brackets) and in the brain. (C, D) Dorsal view of $m d k a$ WM-ISH (C) and $\operatorname{Tg}(e n h: G F P$ ) (D) 2dpf embryos. Arrowheads donate pectoral fins. (E, F) 5dpa caudal fin in WT (E) and $\operatorname{Tg}(e n h: G F P)(\mathrm{F})$. Dashed line = amputation plane. (G-H) $7 \mathrm{dpci} \operatorname{Tg}(w t 1 b: G F P)$ or $T g(e n h: G F P)$ hearts. Injury = marked area. Scale bars, $500 \mu \mathrm{m}$ in $\mathrm{A}, \mathrm{B}, 100 \mu \mathrm{m}$ in $\mathrm{C}-\mathrm{F}, 200 \mu \mathrm{m}$ in $\mathrm{G}-\mathrm{H}$. 


\section{The $\operatorname{Tg}(e n h-m d k a: G F P)$ reporter line}

Given that $\operatorname{Tg}(\mathrm{mdka}: G F P)$ showed GFP expression in the neural tube similar to $\operatorname{Tg}($ enh:GFP), but $\operatorname{Tg}($ enh:GFP) had also stronger GFP expression in the brain and comparable to $m d k a$ WM-ISH, we decided to combine the endogenous promoter of $m d k a$ with the intronic enhancers into the $\mathrm{Tg}$ (enh-mdka:GFP) line. In embryos, GFP was detected as early as $15 \mathrm{hpf}$ (Sup. Figure 3) and later it was expressed in the neural tube and in the brain, equal to the endogenous expression of $m d k a$ with the exception of the pectoral fins (Figure 14A-D) (Video 1). To confirm that GFP expression recapitulated the endogenous $m d k a$ expression, we performed fluorescence ISH for $m d k a$ followed by immunolabelling for GFP (Figure 15). In the brain, $m d k a$ and GFP had an overlapping pattern in the outer layer of the hindbrain, as they did in the neural tube (Figure $\left.15 \mathrm{~A}-\mathrm{B}^{\prime \prime \prime}\right)$. However, the GFP domain was broader, likely due to the proliferation of GFPexpressing cells. Therefore, we examined also 6dpf embryos, where $m d k a$ and GFP were colocalizing in the neural tube (Figure $15 \mathrm{C}-\mathrm{C}^{\prime \prime}$ ). The $\mathrm{GFP}^{+}$cells anatomically resembled glia cells (Kim et al., 2008b). To gain a neural-specific reference, we performed immunofluorescence in $6 \mathrm{dpf}$ embryos for GFP and zns5 that marks the plasma membrane of the cranial ganglia (Trevarrow, 2008). We found GFP expressing cells surrounding inferior otic neuromasts of the anterior lateral line labelled by zns5 (Figure 15D-E', Video 2) (Raible and Kruse, 2000). This observation suggests that these cells are mantle or supporting cells, but further analysis is required to clarify this question (Pistocchi et al., 2009). Overall, $\operatorname{Tg}($ enh-mdka:GFP) expressed GFP in a neural-specific manner during development, recapitulating the endogenous mdka expression.

We continued the analysis by examining the $\operatorname{Tg}($ enh-mdka:GFP) line upon injury (Figure 16). We started with fin amputation, and found that at $3 \mathrm{dpa}$, GFP was detected in the blastema (Figure 16A, C). When compared to $m d k a$ ISH in regenerating fin, GFP resembled $m d k a$ endogenous expression (Figure 16B', C). Additionally, GFP was expressed only after amputation (Sup. Figure 4). Furthermore, we examined GFP expression in control and cryoinjured $\mathrm{Tg}$ (enh$m d k a: G F P$ ) hearts (Figure 16D-G). Cryoinjured hearts expressed GFP in the site of the injury, mimicking the restricted $m d k a$ expression in epicardial cells 7 dpci. Moreover, we did not detect GFP in control hearts, indicating that GFP expression was induced by the injury. We additionally examined 14dpci hearts by immunofluorescence in which GFP was detected in the epicardial cells surrounding the injured area (Figure 16H-I). Since, the $T g($ enh-mdka:GFP) was inducing GFP expression similar to $m d k a$ endogenous expression in development and after injury, we wondered if that was the case for other adult tissues that express $m d k a$. Therefore, we examined GFP expression in the brain and found a well-defined GFP signal in the surface of the telencephalon (Tel), habenula (Ha), commissura tectil (Ctec), tectum opticum (TeO), corpus cerebelli (CCe), crista cerebellaris (CC) and medulla oblongata (MO) (Figure 17A, B) (Wullimann et al., 1996). Additionally, strong signal was detected in the inferior lobus of the hypothalamus (Figure 17C, $\mathrm{C}^{\prime}$ ). Hence, we found that GFP expression recapitulated the endogenous $m d k a$ gene expression pattern 
(Winkler et al., 2003). We further analysed the expression by immunolabelling of Mdka and GFP in brain sections, and we found that the GFP and Mdka overlapped in the outer layer of different brain areas including the olfactory bulb (OB) and the inferior lobe of the hippopotamus (IL) (Figure 17D-D"', brackets). Interestingly, Mdka and GFP were co-localized in what appeared to be the vasculature, however further analysis will clarify this finding (Figure 17D'-D'", arrows). Moreover, GFP was detected in the horizontal cells of the outer nuclear layer (ON) in adult retina (Figure 17E-E'), an expression pattern that remains stable through ageing, since $m d k a$ had the same expression in 3dpf retina (Figure 17F-F') and it is in agreement with published expression data (Gramage et al., 2015). Taken together, our data show that the $m d k a$ promoter in combination with the enh region are able to recapitulate $m d k a$ endogenous expression in developing embryos, in adult tissues and in the heart and the fin after injury.
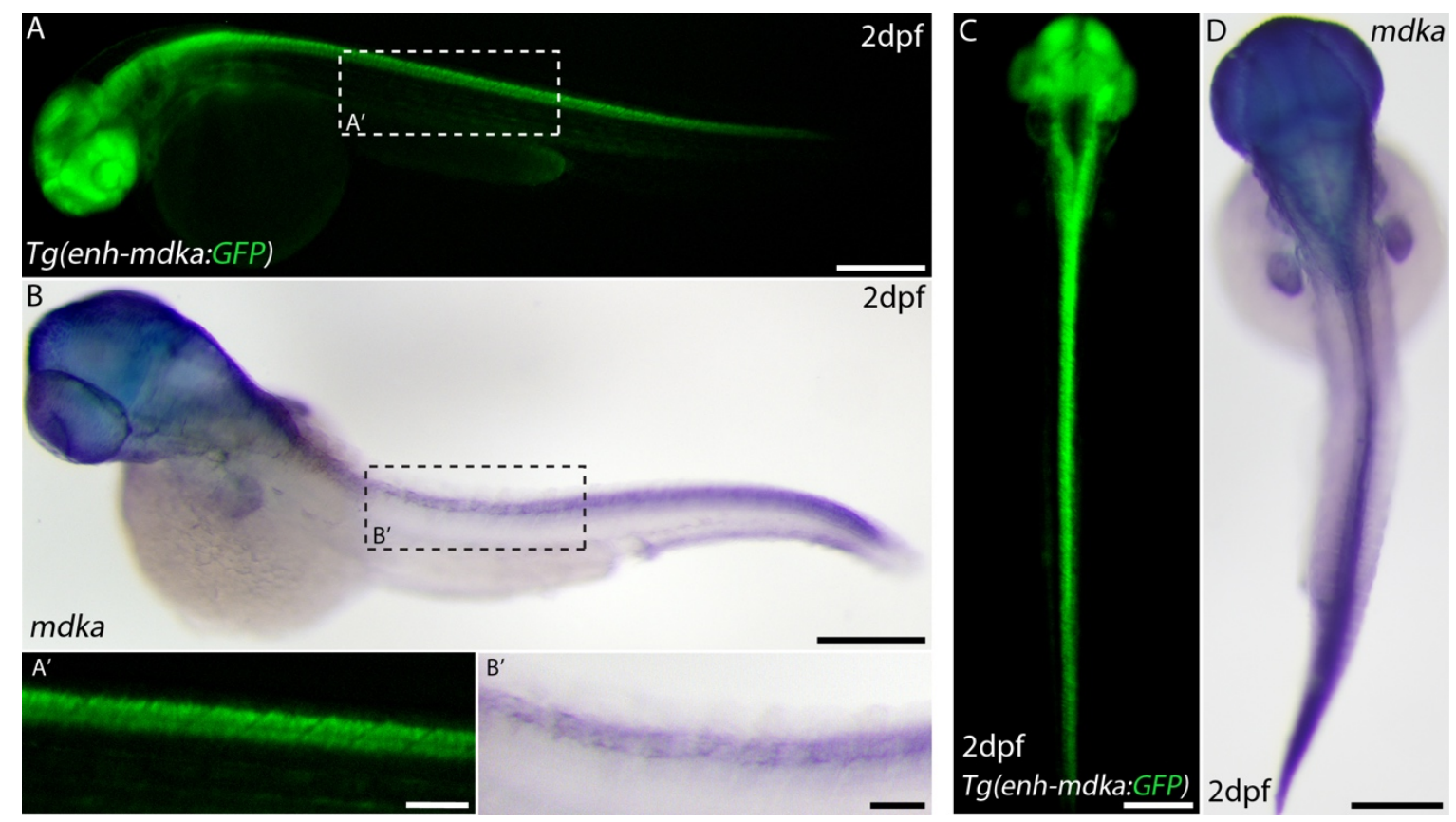

Figure 14. $\mathrm{Tg}($ enh-mdka:GFP) expression in embryos
(A) Lateral view of $2 \mathrm{dpf} T g(e n h-m d k a: G F P)$ embryo.
(B) WM-ISH for $m d k a$ in 2 dpf embryo. (A', B') Higher magnifications of the dashed areas in A and B. Dorsal view of $\operatorname{Tg}($ enh-mdka:GFP) (C) and $m d k a$ WM-ISH (D) 2dpf embryos. Scale bars, $200 \mu \mathrm{m}$ in A, B,C and D, $50 \mu \mathrm{m}$ in A' and B'. 

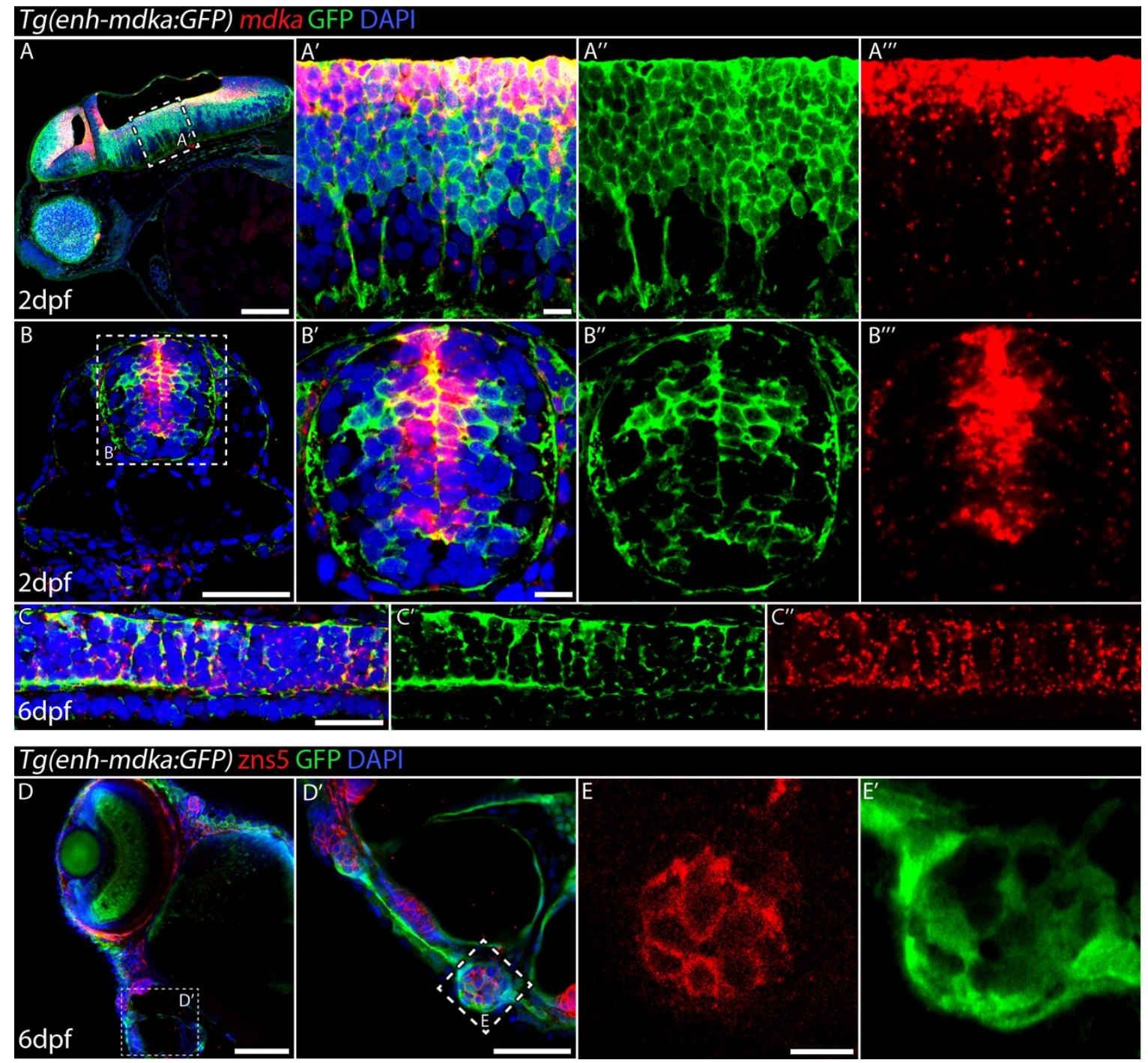

Figure 15. Expression analysis of $T g(e n h-m d k a: G F P)$ embryos by FISH/IF

(A-C") Fluorescence ISH for $m d k a$ coupled by GFP immunostaining. (A) Lateral view of $2 \mathrm{dpf}$ $\operatorname{Tg}($ enh-mdka:GFP) embryo. (A'-A'") Higher magnification of the marked area of the hindbrain in A. (B) Transverse section of $2 \mathrm{dpf} \operatorname{Tg}($ enh-mdka:GFP) trunk, showing the neural tube. (B'-B'"') Magnification of the dashed area in B. (C-C') Image of the neural tube of $6 \mathrm{dpf} \operatorname{Tg}($ enh-mdka:GFP). (D-E') Whole-mount immunolabelling of GFP and zns5 in 6dpf Tg(enh-mdka:GFP) embryos. (D') Magnification of the marked area in D. (E-E') Higher power of the marked area in D'. Scale bars, $100 \mu \mathrm{m}$ in $\mathrm{A}, \mathrm{D}, 50 \mu \mathrm{m}$ in $\mathrm{B}$ and $\mathrm{D}^{\prime}, 25 \mu \mathrm{m}$ in $\mathrm{C}^{-\mathrm{C}^{\prime \prime}}, 10 \mu \mathrm{m}$ in $\mathrm{A}^{\prime}-\mathrm{A}^{\prime \prime \prime}, \mathrm{B}^{\prime}-\mathrm{B}^{\prime \prime \prime}, \mathrm{E}$ and $\mathrm{E}^{\prime}$ 

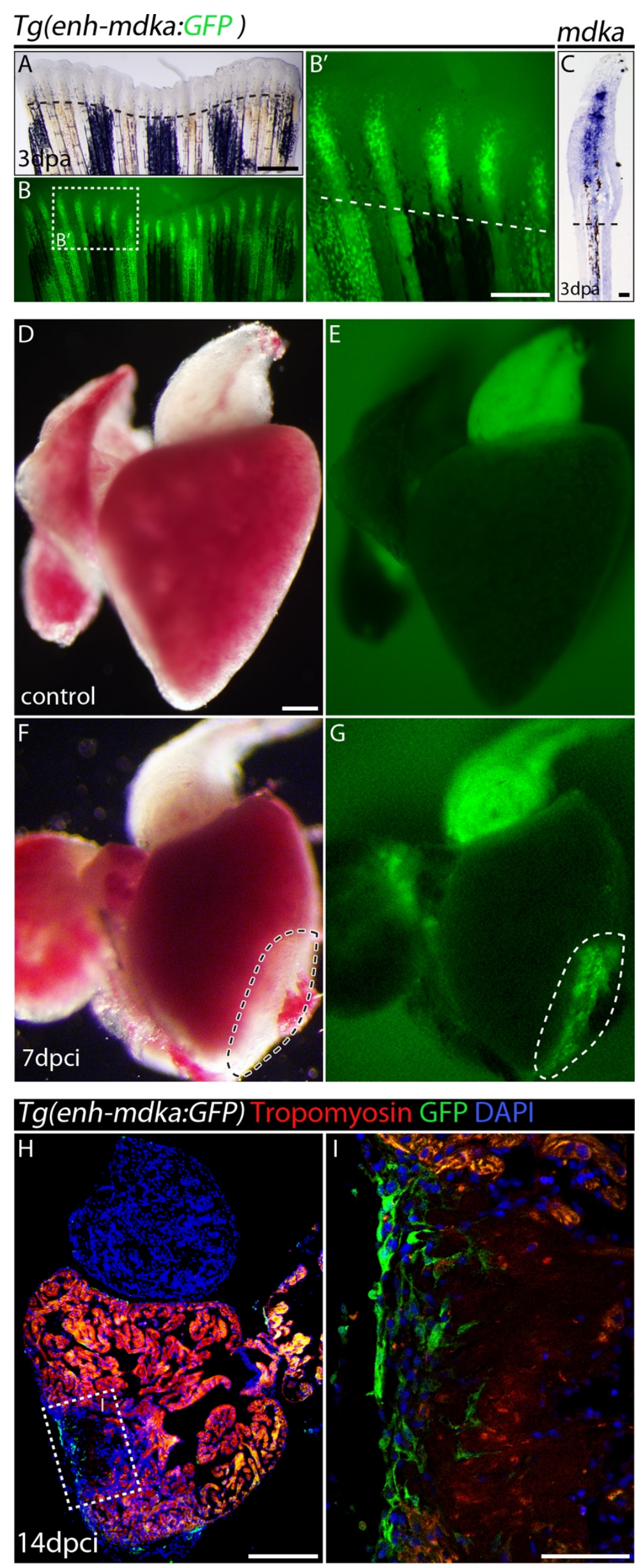

Figure 16. Analysis of adult Tg(enh-mdka:GFP) upon injury

Brightfield (A) and fluorescent (B) images of $3 \mathrm{dpa}$ caudal fin. (B') Higher magnification of the selected area in B. (C) ISH of $m d k a$ on 3 dpa fin section. Dashed lines indicate the amputation plane. (D, E) Whole mount images of control (not injured) $\quad \operatorname{Tg}($ enh-mdka:GFP) heart, brightfield (D) and fluorescent (E). (F, G) Whole mount images of 7dpci $\mathrm{Tg}$ (enh$m d k a: G F P$ ) heart, brightfield (F) and fluorescent $(\mathrm{G})$. Dashed area $=$ injury. $(\mathrm{H})$ GFP staining in $\operatorname{Tg}($ enh-mdka:GFP) $\quad$ 14dpci heart. (I) Magnification of the marked area in H. Scale bar, $200 \mu \mathrm{m}$ in $\mathrm{D}-\mathrm{G}, 100 \mu \mathrm{m}$ in the rest. 

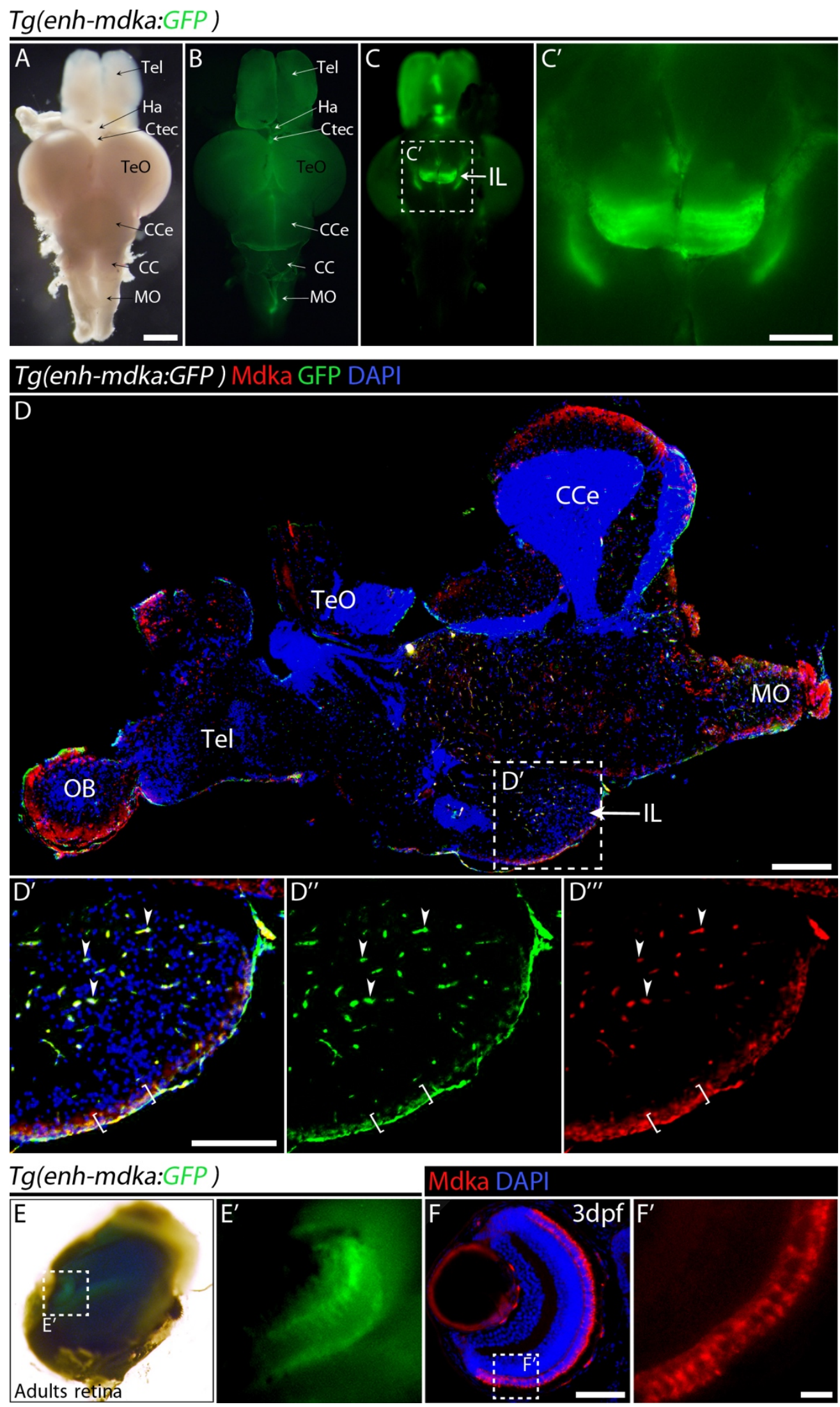

Figure 17. Examination of the GFP expression in $T g(e n h-m d k a$ :GFP) adult neural tissues (A, B) Dorsal view of brain brightfield or fluorescent. Tel = telencephalon, $\mathrm{Ha}=$ habenula, $\mathrm{Ctec}=$ commissura tectil, $\mathrm{TeO}=$ tectum opticum, $\mathrm{CCe}=$ corpus cerebelli, $\mathrm{CC}=$ crista cerebellaris, $\mathrm{MO}=$ 
medulla oblongata, (IL) = inferior lobe of the hippopotamus. (C) Ventral view of $\operatorname{Tg}(e n h$ mdka:GFP) brain. Marked area indicates the lobus inferior (IL) of hypothalamus. (C') Magnification of the selected area in C. (D) Immunostaining of Mdka and GFP in section of adult brain. $\mathrm{OB}=$ olfactory bulb $(\mathrm{OB})$. ( $\left.\mathrm{D}^{\prime}\right)$ Magnification of the dashed area in $\mathrm{D}$. brackets indicate the GFP-Mdka overlapping in the outer layer of the brain, and arrows in internal structures (E) Brightfield view of $T g(e n h-m d k a: G F P)$ adult retina. (E') Magnification of the GFP expression in E. (F) Immunostaining of $3 \mathrm{dpf}$ retina section for Mdka. $\left(\mathrm{F}^{\prime}\right)$ Magnification of the marked area in $\mathrm{E}$. Scale bars, $500 \mu \mathrm{m}$ in A-C, $200 \mu \mathrm{m}$ in $\mathrm{C}^{\prime}, 100 \mu \mathrm{m}$ in $\mathrm{D}, 50 \mu \mathrm{m}$ in $\mathrm{F}, \mathrm{D}^{\prime}-\mathrm{D}^{\prime \prime \prime}$ and $10 \mu \mathrm{m}$ in $\mathrm{F}^{\prime}$.

\section{$\operatorname{Tg}(\operatorname{Reg}: G F P)$ analysis}

Next, we analysed the transgenic fish carrying the Reg genomic region. One-day post fertilization, GFP was expressed in cells of the outer layer of the brain (Figure 18A, B, Video 3). This distinct expression became clearer in $36 \mathrm{hpf}$ embryos analysed by confocal microscopy and 3D volume rendering, that revealed GFP expression also in the midline of the head (Figure 18C, arrow in $\mathrm{D}$, video 4,5 ). The $\mathrm{GFP}^{+}$cells covering the roof of the hindbrain ventricle resembled epithelial cell of the choroid plexus (CP) (Van Leeuwen et al., 2018) that are important for the formation of the blood-cerebrospinal fluid (CSF) barrier (Obermeier et al., 2013). GFP was also detected in the spinal tube and in the axons of motor neurons (Figure 18E, brackets and arrowheads). The expression in the neural axons is similar to the $T g(m d k a: G F P)$ and $T g(e n h: G F P)$ expression, and in contrast to mdka endogenous expression. Nevertheless, to gain a clear view of the GFP longitudinal expression, we crossed the $\operatorname{Tg}(\operatorname{Reg}: G F P)$ to the $T g(f l i l a: D s R e d e x)$ line that expresses DsRed in the vasculature and analysed whole-mount $\operatorname{Tg}(\operatorname{Reg}: G F P) / T g(f l i 1 a: D s R e d e x)$ embryos under a confocal microscope (Figure 19A). 3D volume rendering showed clearly that GFP was expressed in the spinal tube and in neuron's bodies and axons expanding dorsally 2dpf (Figure 19AC, brackets, arrowheads and arrows, respectively, Video6, 7). Similarly, at 6dpf (Figure 19D-F) the GFP signal in the lateral line was strong, as it was in the neural tube (Figure 19D brackets, E yellow arrowheads, F, Video 8, 9). To confirm GFP expression in the neural axons, we immunolabeled GFP together with zns5 on whole-mound $\operatorname{Tg}($ Reg:GFP) embryos (Figure 20). We found GFP associated to the $\mathrm{zns} 5^{+}$axons of motor neurons derived from the spinal tube and not with their bodies (Figure 20A-B"'). Additionally, GFP was detected covering the branches of the otic and supraorbital cranial neuromasts (Figure 20C-G, Video 10) (Raible and Kruse, 2000) but not the ganglia, as we showed in the $T g(e n h-m d k a: G F P)$ line. We had the same results when we tested other neural-specific antibodies (zn3, zn12, Sup. Figure 5), reinforcing the idea that the $\mathrm{GFP}^{+}$cells are glia supporting cells. To address if the Reg genomic region was a regeneration-related enhancer, we examined GFP expression in adult tissues after injury. Three-days post amputation, the regenerating fin expressed weak GFP in the blastema and with less intensity than the $\operatorname{Tg}(e n h$ $m d k a: G F P$ ) fins (Figure 21A, arrows). Control hearts however, had no GFP expression, conversely 
to $7 \mathrm{dpci}$ where GFP was detected in the ventricle, presumable in CM, but not in the site of the injury (Figure 21A, $\mathrm{C}^{\prime}$ ). Suggesting that GFP expression is activated upon injury but in different cells than $m d k a$ endogenous expression. Overall, Reg drove the expression of GFP in the developing spinal tube similar to $m d k a$, and in the brain, in a restricted expression pattern. However, GFP was detected in the axons of the motor neurons where $m d k a$ is not detectable by WM-ISH. Upon fin or heart injury, GFP was detected although either the signal intensity was trivial or in areas not associated to the endogenous $m d k a$ expression.

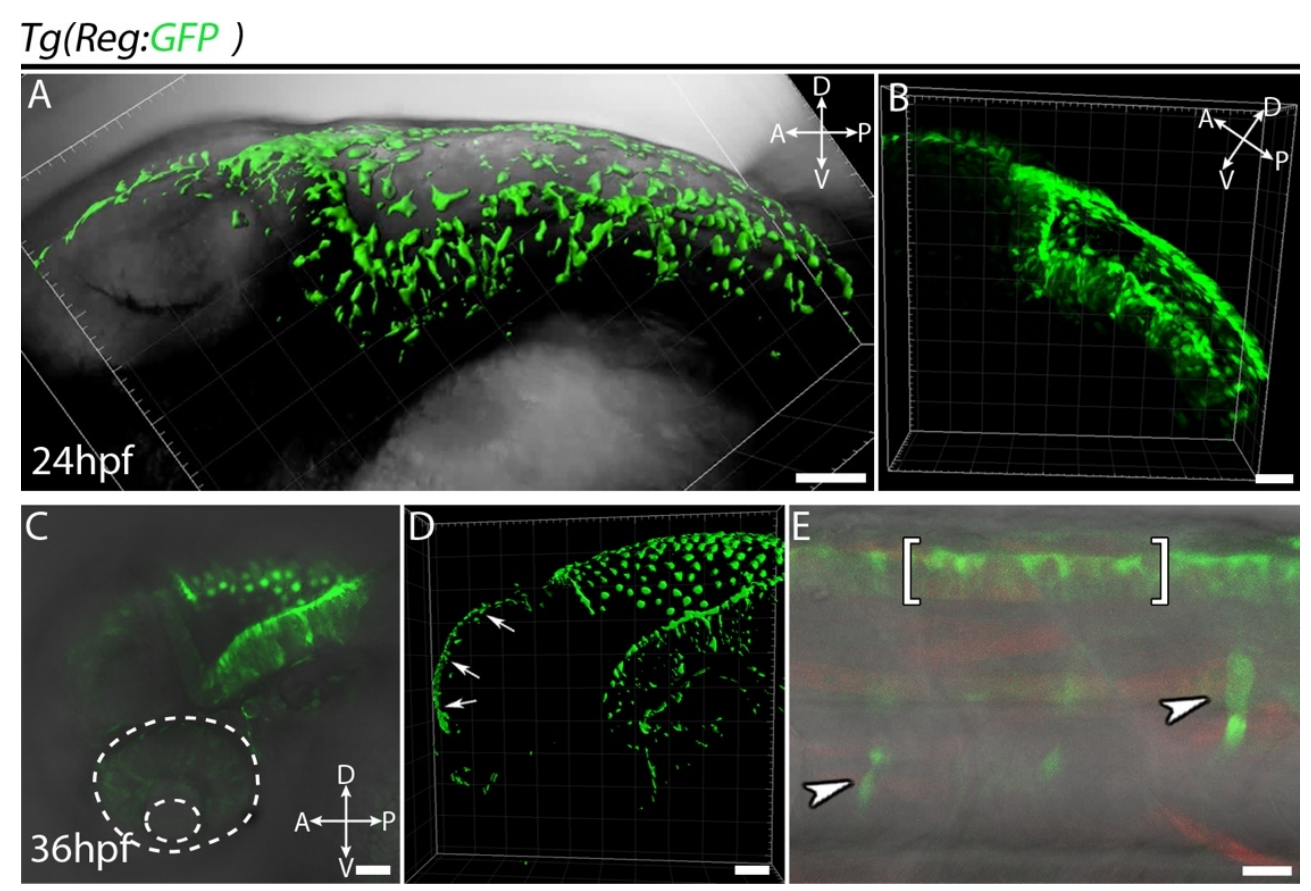

Figure 18. $\operatorname{Tg}(\operatorname{Reg}: G F P)$ expression in embryos

(A, B) 3D volume rendering merge to brightfield and 3D reconstruction of $24 \mathrm{hpf} \operatorname{Tg}(\operatorname{Reg}: G F P)$ embryo. (C, D) Confocal image merge to brightfield and 3D volume rendering of 36hpf embryo brain. Dashed lines indicate the eye, arrows the midline. (E) Merge of fluorescence and brightfield images of the trunk showing GFP expression in the neural tube (brackets) and in the neural axons (arrowheads). DsRed expression is also visible from the X.Card.Actin:DsRed vector. $\mathrm{A}=$ anterior, $\mathrm{P}=$ posterior, $\mathrm{D}=$ dorsal, $\mathrm{V}=$ ventral. Scales, $50 \mu \mathrm{m}$ in $\mathrm{A}, \mathrm{B} ; 70 \mu \mathrm{m}$ in $\mathrm{C}, \mathrm{D}, 20 \mu \mathrm{m}$ in $\mathrm{E}$. 


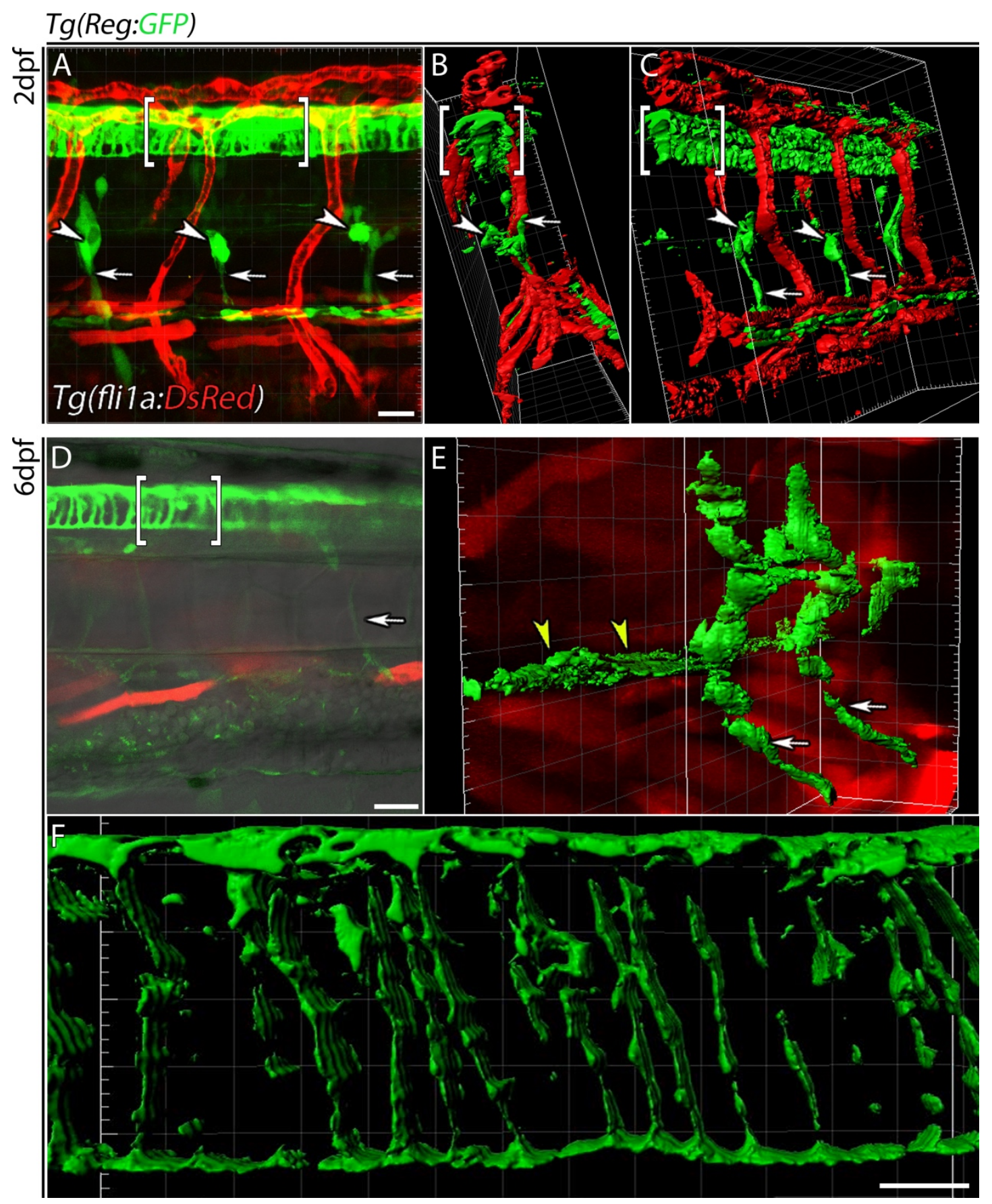

Figure 19. 3D analysis of $T g(\operatorname{Reg}: G F P)$ embryos

(A) Lateral view of the trunk showing GFP expression in the spinal tube (brackets) and in the axons bodies (arrowheads) and axons (arrows) and DsRed expression in the vasculature of a $2 \mathrm{dpf}$ $\operatorname{Tg}$ (Reg:GFP)/Tg(flila:DsRedex) embryo. (B, C) 3D volume rendering of the panel A in transvers and lateral view. Brackets indicate the spinal tube, arrowheads the bodies and arrows the axons. (D) Merge of confocal fluorescence and brightfield images of the trunk of $\operatorname{Tg}(\operatorname{Reg}: G F P)$ embryo 6dpf, brackets indicate the neural tube and the arrow the neural axon. (E, F) 3D volume rendering of the panel D showing the lateral line (yellow arrowheads) and the neural axons (arrows) as well as the spinal tube (F). DsRed muscle cells are visible because of the X.Card.Actin:DsRed cassette. Scale bars, A $20 \mu \mathrm{m}, \mathrm{D} 30 \mu \mathrm{m}, \mathrm{F} 10 \mu \mathrm{m}$. 

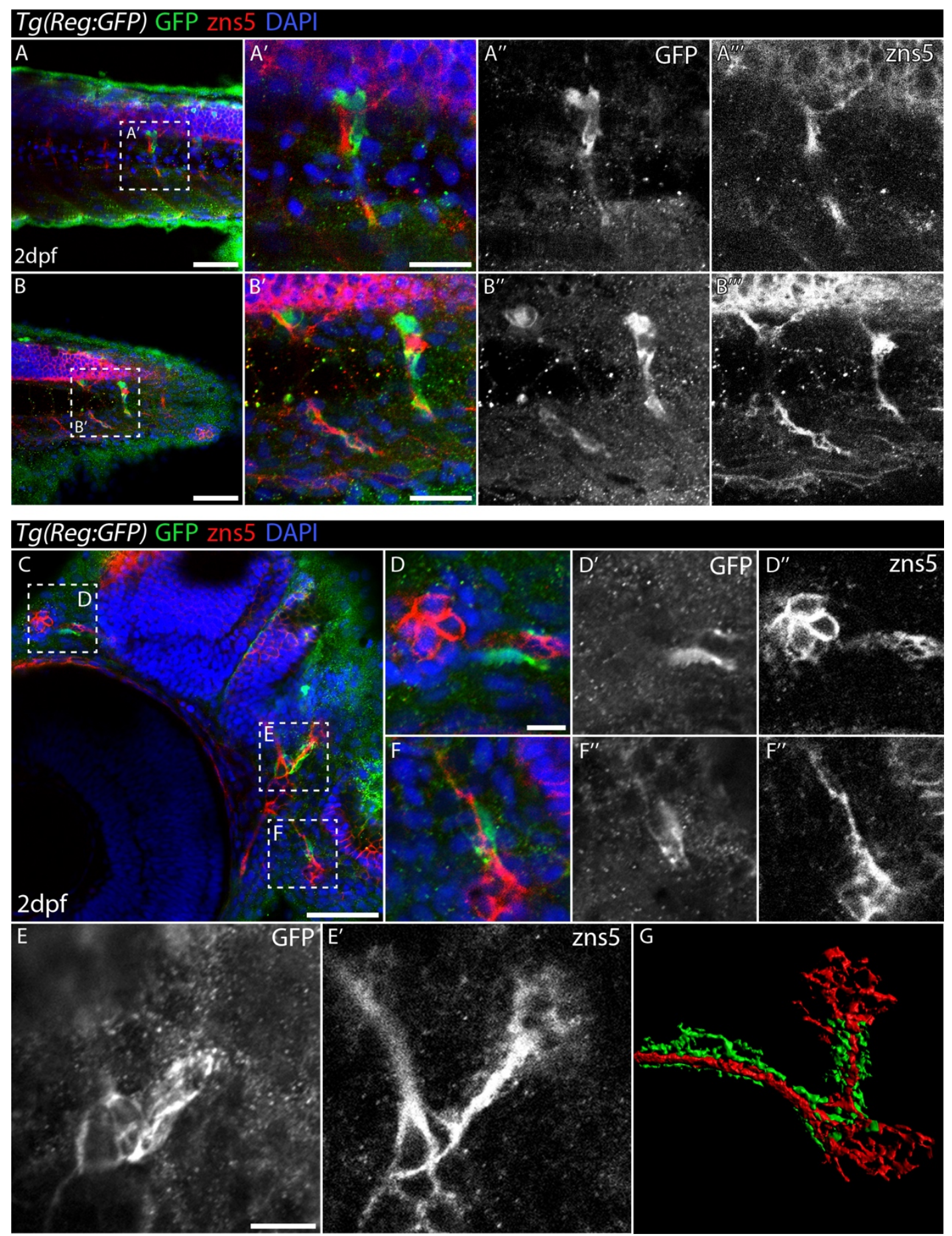

Figure 20. Immunofluorescence analysis of $T g(\operatorname{Reg}: G F P)$ embryos

(A-G) Whole-mount immunostaining of GFP and zns5 of 2dpf embryos illustrating the association of GFP with zns5 $5^{+}$neural axons. ( $\left.\mathrm{A}^{\prime}-\mathrm{A}^{\prime \prime \prime}, \mathrm{B}^{\prime}-\mathrm{B}^{\prime \prime \prime}\right)$ Higher magnification of the boxed areas in A and B. (C-G) Images showing the cranial neuromasts in red and the GFP expression. (D, E, F) Magnifications of the selected areas in C. (G) 3D volume rendering of the panel E. Scale bars, $50 \mu \mathrm{m}$ in $\mathrm{A}, \mathrm{B}$ and $\mathrm{C}, 25 \mu \mathrm{m}$ in $\mathrm{A}^{\prime}-\mathrm{A}^{\prime \prime \prime}, 10 \mu \mathrm{m}$ in D-D"', E, E', F-F"'. 

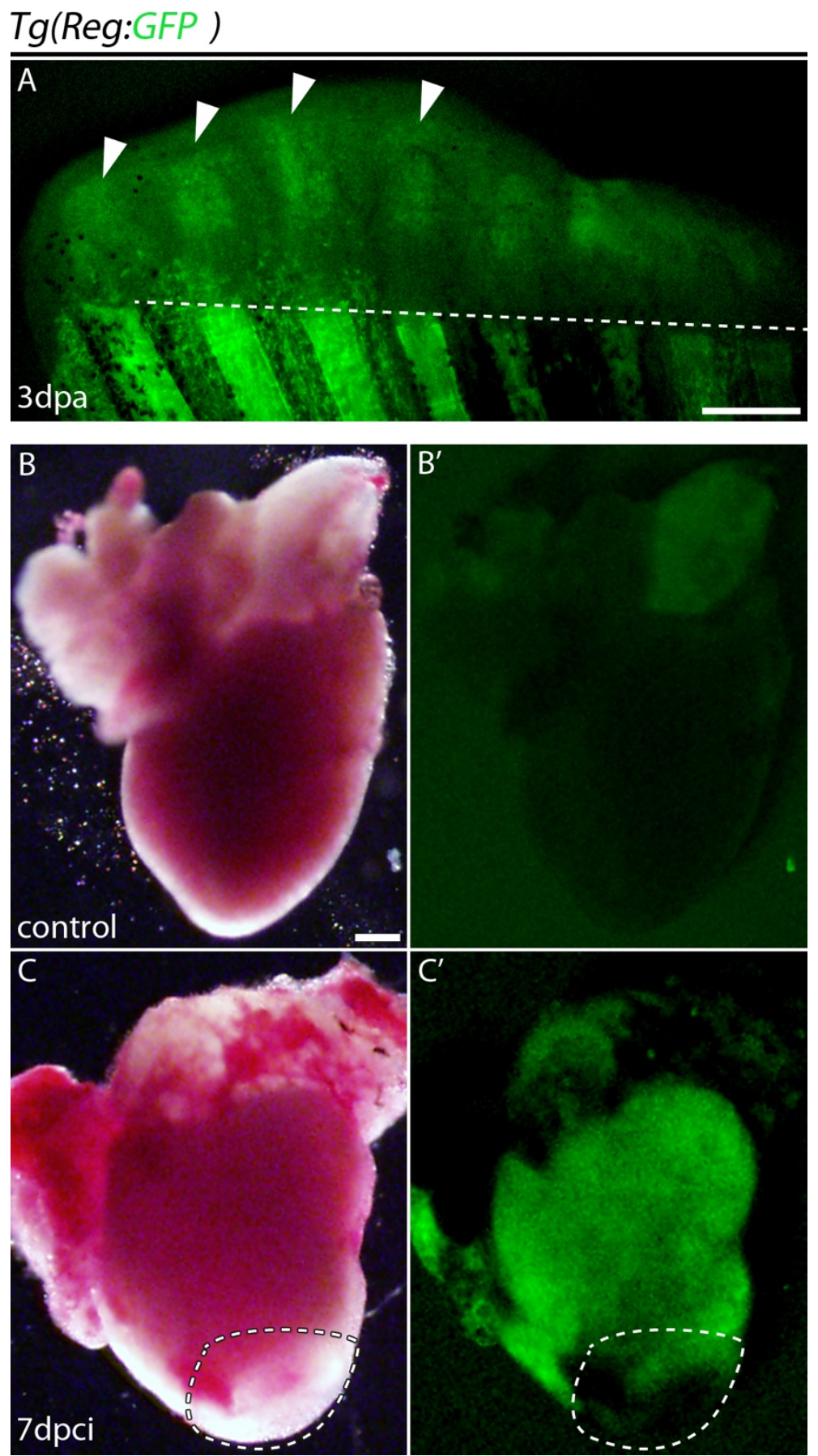

Figure 21. $\operatorname{Tg}(\operatorname{Reg}: G F P)$ after injury

(A) 3dpa fin showing minimal GFP expression (arrows). Whole-mount images of control (B-B') and $7 \mathrm{dpci}\left(\mathrm{C}-\mathrm{C}^{\prime}\right)$ hearts. Dashed lines donate amputation plane in A and injured area in $\mathrm{C}, \mathrm{C}^{\prime}$. Scale bars, $100 \mu \mathrm{m}$ in A and $200 \mu \mathrm{m}$ in the rest. 


\section{Enhancer-4 and enhancer-5 in development and regeneration}

To complete the analysis of $m d k a$ epigenetic landscape we analysed also the $\operatorname{Tg}($ enha4:GFP) and $\operatorname{Tg}($ enha5:GFP) transgenic animals. Two-days post fertilization, $\operatorname{Tg}$ (enha4:GFP) embryos expressed GFP in the brain, eye and in the neural axons and interestingly, also in the heart (Figure 22A arrow, B arrowheads). To analyse this cardiac expression, we crossed $\operatorname{Tg}$ (enha4:GFP) to $\operatorname{Tg}(f l i 1 a: D s R e d e x)$ or to $\operatorname{Tg}(m y l 7: m R F P)$ that expresses PRF targeted to the membranes of CM. We found GFP overlapping with some endothelial DsRed ${ }^{+}$cells in the embryonic heart but also in surrounding cells that were CM (Figure 22C-C"', Figure 22D-D' '). This observation suggested that 2dpf enha4 was driving the GFP expression in specific endocardial cells and in CM. However, 6dpf the GFP expression was restricted in the forming valves without overlapping with CM (Figure 22E-H'). In adults, GFP was detected only in cryoinjured hearts, and further analysis will reveal in which cell type is expressed (Figure 22I-J'). However, in fins 3dpa, GFP co-localized with endothelial DsRed (Figure 22K-L").

The $\operatorname{Tg}($ enha5:GFP) embryos had strong GFP signal in the eye, the pectoral fin and in the heart (Figure 23A, A'). To examine in which cardiac cell type GFP was expressed, we crossed the line to $\operatorname{Tg}(f l i 1 a: D s R e d e x)$ or $\operatorname{Tg}(m y l 7: m R F P)$. We detected GFP in the CM of the embryonic heart $3 \mathrm{dpf}$ surrounded by the membrane RFP (Figure 23B, B'). The CM specific expression of GFP was also confirmed by the $\operatorname{Tg}\left(\right.$ enha5:GFP)/Tg(flila:DsRedex) double transgenic, where the DsRed ${ }^{+}$ endocardium was adjacent to the $\mathrm{GFP}^{+} \mathrm{CM}$ (Figure 23C-C'). Likewise, adult control hearts were expressing GFP in CM of the ventricle and the atrium, similarly to the situation in embryos (Figure 23D-D'). In conclusion, enha4 and enha5 partially drive GFP in tissues that $m d k a$ is expressed such as the brain and the pectoral fin. Interestingly, GFP expression was detected in CM and in the endocardium, two cell types in which $m d k a$ is not expressed during development. Additionally, enha4 has a dynamic expression pattern during cardiogenesis, since GFP was transiently expressed in $\mathrm{CM}$ and endocardium at $2 \mathrm{dpf}$, and switched to the valves by $6 \mathrm{dpf}$. 


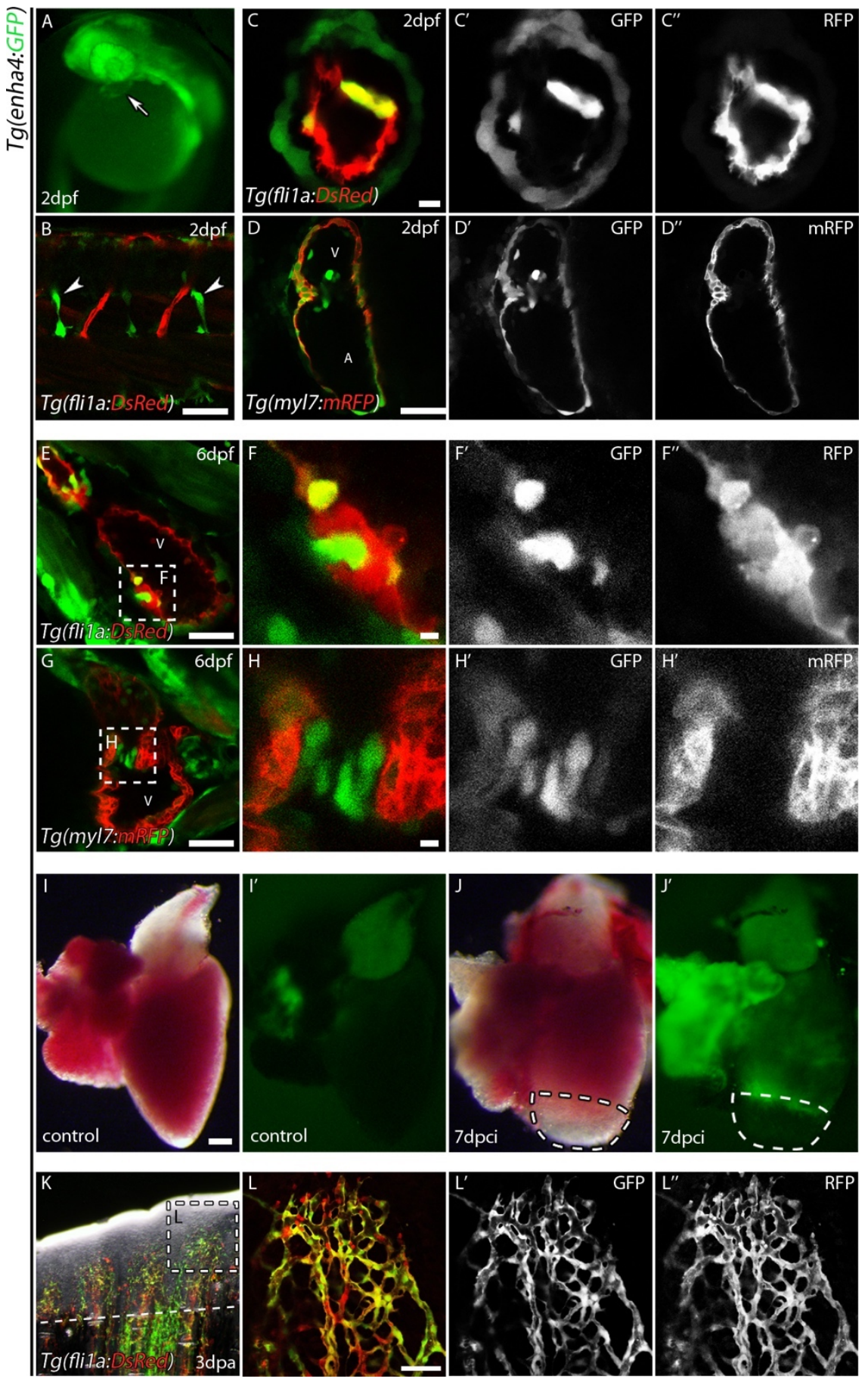

Figure 22. $\operatorname{Tg}(e n h a 4: G F P)$ analysis

(A) $2 \mathrm{dpf}$ embryos presented GFP in the heart (arrow). (B) Image of the trunk of $\operatorname{Tg}$ (enha4:GFP)/Tg(flila:DsRedex) embryo. Fluorescence images of Tg(enha4:GFP) $/ T g(f l i 1 a: D s R e d e x)$ heart $2 \mathrm{dpf} \quad\left(\mathrm{C}-\mathrm{C}^{\prime \prime}\right)$ and $6 \mathrm{dpf} \quad\left(\mathrm{E}-\mathrm{F}^{\prime \prime}\right)$. Fluorescent image of $\operatorname{Tg}$ (enha4:GFP)/Tg(myl7:mRFP) heart 2dpf (D-D' ') and 6dpf (G-H'). Control (I-I') and 7dpci (J$\left.\mathrm{J}^{\prime}\right) \operatorname{Tg}($ enha4:GFP) adult hearts, dashed areas = injury. (K-L'") 3dpa $\operatorname{Tg}($ enha4:GFP)/ $\operatorname{Tg}$ (flila:DsRedex) fin, dashed line = amputation plane. (L-L") Higher magnification of the selected

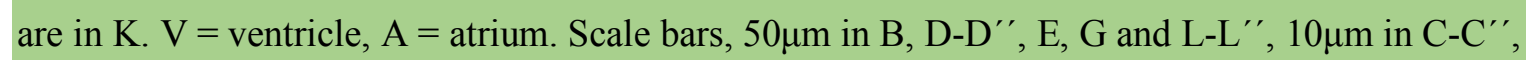
F-F"', H-H', $200 \mu \mathrm{m} \mathrm{I-J'}$. 


\section{Tg(enha5-mdka:GFP)}
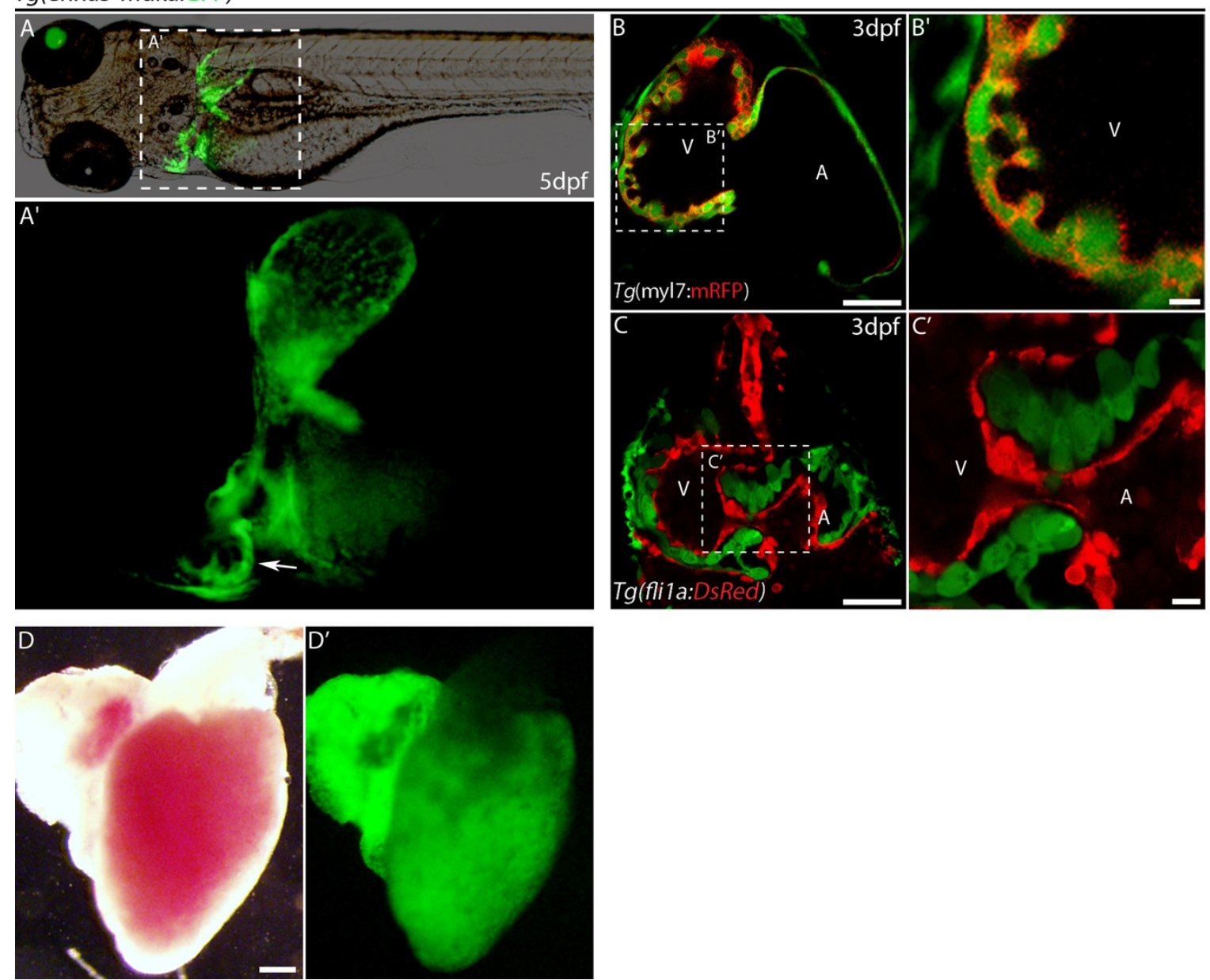

Figure 23. $\operatorname{Tg}($ enha5:GFP) analysis

(A) Lateral view of $5 \mathrm{dpf} \operatorname{Tg}($ enha5:GFP) embryo. (A') Magnification of the dashed area in A, arrow indicates the heart. (B-B') Image of a $3 \mathrm{dpf} \operatorname{Tg}($ enha4:GFP)/Tg(myl7:mRFP) heart. (C-C' $)$ Image of a 3dpf $\operatorname{Tg}($ enha4:GFP)/Tg(flila:DsRedex) heart. (D-D') Whole-mount images of $\operatorname{Tg}\left(\right.$ enha4:GFP) hearts. $\mathrm{V}=$ ventricle, $\mathrm{A}=$ atrium. Scale bars, 50 in $\mathrm{B}, \mathrm{C}, 10 \mathrm{in} \mathrm{B}^{\prime}, \mathrm{C}^{\prime}, 200 \mu \mathrm{m}$ in $\mathrm{D}, \mathrm{D}^{\prime}$. 


\section{Transcription factors analysis on $m d k a$ cis-regulatory elements}

The in vivo evaluation of the $m d k a$ cis-regulatory elements revealed the minimum regulatory genomic regions that drive the expression in development, adults and after injury, the mdka-enh sequences. Additionally, we detected enhancers, enha4 and enha5, that induced GFP expression where $m d k a$ is not expressed, including CM and valves mesenchyme. Therefore, we have investigated the transcription factors (TF) that bind to each regulatory regions and may explain the GFP expression pattern that we detected (Table7) (Grant et al., 2011; Khan et al., 2018). We found TF predicted to bind to more than one region such as Is12, Mef2D and Rxra, that are expressed in the central nervous system (CNS) during development (Hammond and Udvadia, 2010; Moreno et al., 2018; Tallafuss et al., 2006), as well as unique TF, including Hif1a, Nr2e3 and Nfact2. The analysis also predicted Nfatc2, Nkx2-5 and Mef2A in $\operatorname{Tg}($ enha4:GFP) and $\operatorname{Tg}($ enha5:GFP), explaining the CM expression we detected, also in the case of $\operatorname{Tg}(\operatorname{Reg}: G F P)$, although it was only detected in adult animals (Hammond and Udvadia, 2010; Zhang et al., 2018). Because we found strong expression of $\operatorname{Tg}($ enha4:GFP) in endothelial cells after fin amputation, we analysed enha4 for endothelial TF. The analysis predicted that F11a, Gata2 and Erg [reviewed in (Park et al., 2013)] have binding sites in enha4, clarifying the endothelial expression we detected.

Table 7. TF binding sites in $m d k a$ cis-regulatory elements

\begin{tabular}{|c|c|c|}
\hline & TF & p-value \\
\hline \multirow{9}{*}{ enh-mdka } & Creb1 & $1,47 \mathrm{E}-05$ \\
\hline & Foxd1 & $2,18 \mathrm{E}-05$ \\
\hline & Hifla & $3,82 \mathrm{E}-05$ \\
\hline & Is12 & $1,79 \mathrm{E}-05$ \\
\hline & Max & $8,37 \mathrm{E}-05$ \\
\hline & Mef2D & $9,03 \mathrm{E}-05$ \\
\hline & Rxra & $9,67 \mathrm{E}-05$ \\
\hline & Sox19 & $2,99 \mathrm{E}-06$ \\
\hline & Spil & $6,51 \mathrm{E}-05$ \\
\hline \multirow{6}{*}{ Reg } & Is12 & $7,22 \mathrm{E}-05$ \\
\hline & Nkx2-5 & $9,66 \mathrm{E}-05$ \\
\hline & Mef2A & $5,16 \mathrm{E}-05$ \\
\hline & Mef2D & $5,55 \mathrm{E}-05$ \\
\hline & Nr2e3 & $6,51 \mathrm{E}-05$ \\
\hline & Dlx2 & 7,93E-05 \\
\hline
\end{tabular}

\begin{tabular}{|c|c|c|c|}
\hline & & TF & p-value \\
\hline \multirow{10}{*}{ enha4 } & & Fos::Jun & $6,51 \mathrm{E}-05$ \\
\hline & & Spil & $6,51 \mathrm{E}-05$ \\
\hline & & Soc19 & $2,42 \mathrm{E}-05$ \\
\hline & & Max & $3,01 \mathrm{E}-06$ \\
\hline & & Nkx2-5 & $9,66 \mathrm{E}-05$ \\
\hline & & Rxra & $4,40 \mathrm{E}-05$ \\
\hline & \multirow{4}{*}{ 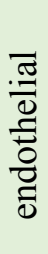 } & Ewsr1::Fli1 & $3,80 \mathrm{E}-05$ \\
\hline & & Fli1 & $3,27 \mathrm{E}-05$ \\
\hline & & Gata2 & $4,96 \mathrm{E}-06$ \\
\hline & & Erg & $1,61 \mathrm{E}-05$ \\
\hline \multirow{7}{*}{ enha5 } & & Is12 & $7,22 \mathrm{E}-05$ \\
\hline & & Nkx2-5 & $9,66 \mathrm{E}-05$ \\
\hline & & Mef2A & $1,64 \mathrm{E}-06$ \\
\hline & & NfatC2 & $7,93 \mathrm{E}-05$ \\
\hline & & Mef2D & 4,94E-07 \\
\hline & & Dlx2 & 7,93E-05 \\
\hline & & Rxra & $5,60 \mathrm{E}-05$ \\
\hline
\end{tabular}




\section{Caveolin-1 in heart regeneration}

\section{Caveolin-1 expression in the heart}

CAV1 expression has been reported in variety of cell lines (Cohen et al., 2003; Mora et al., 1999; Patel et al., 2007; Robenek et al., 2008a; Scherer et al., 1997), hence we started by examining the expression in control and injured adult heart. We used the $\operatorname{Tg}(f l i l a: G F P)$ transgenic line (Lawson and Weinstein, 2002) that expresses GFP in the adult endocardium and endothelium, and simultaneously stained with antibodies against Cav1 and Tropomyosin, to label the CM (Figure $\left.24 \mathrm{~A}-\mathrm{C}^{\prime \prime}\right)$. Robust expression was detected in the vasculature of the cortical myocardium (asterisks in Figure 24B-B") and in the endocardium overlying the trabecular CM (arrowheads in Figure 24B-B" and Figure 24C-C"). Strong expression was also found in the epicardium (arrows in Figure $24 \mathrm{~B}-\mathrm{B}^{\prime \prime}$ ), in the bulbus arteriosus and in the valves (ba and $\mathrm{v}$ in Figure 24A, respectively). Additional, Cav1 expression was observed between the cortical and trabecular myocardium (doted area in Figure 24B-B" and insert in Figure 24B'). We also detected endocardial and bulbus arteriosus expression of Cav1 in the $3 \mathrm{dpf}$ developing heart (Sup. Figure 6A-A'). We have also examined Cav1 expression in wild type embryonic mouse hearts (Sup. Figure 6B-J). At E15,5, Cav1 was expressed in coronary endothelium, endocardium, and in the epicardium (asterisks in Sup. Figure 6F, arrowheads in Sup. Figure 6D, E and arrows in Sup. Figure 6F, respectively). The epicardial, endocardial, and endothelial expression of Cav1 in the mouse heart was confirmed by immunofluorescent labelling (Sup. Figure 6G-J); in which, CAV1 overlapped with isolectinB4 (ISB4) that labels endothelium, endocardium and epicardium (asterisks in Sup. Figure 6J, arrowheads in Sup. Figure 6I and arrows in Sup. Figure 6J, respectively). We then analysed Cav1 expression in regenerating zebrafish heart (Figure 24D). For that, we used the $T g(w t 1 b: G F P)$ line that expresses GFP in the epicardium upon injury (González-Rosa et al., 2012). Seven-days post cryoinjury, Cav1 was expressed strongly in epicardial cells (brackets in Figure 24E and Figure 24F$F^{\prime}$ ) covering the injured site, overlapping with GFP. High expression was also detected in the endocardium inside the injured area (arrows in Figure 24F). To confirm the endocardial expression inside the injured area we used the $\operatorname{Tg}(f l i l a: G F P)$ line in which Cav1 overlapped with GFP in the endocardium invading the damaged tissue (Figure 24G-H' arrows). Moreover, we analysed the expression of caveolae-related genes during regeneration by qPCR (Figure 24I). cav 1 and cavin 1 b were upregulated after cardiac injury, in contrast to the cav2 and cav3, whose expression did not change during the first days of regeneration. Cav1 expression in the undamaged adult zebrafish heart is abundant in different cell types, including the endocardium, endothelium and epicardium, three cardiac cell types that are activated during regeneration (Gonzalez-Rosa et al., 2011; Kikuchi et al., 2011c; Marín-Juez et al., 2016; Münch et al., 2017). Yet, Cav1 was upregulated upon cardiac injury, highly expressed by epicardial cells surrounding the injured site and endocardium invading the damaged tissue. 

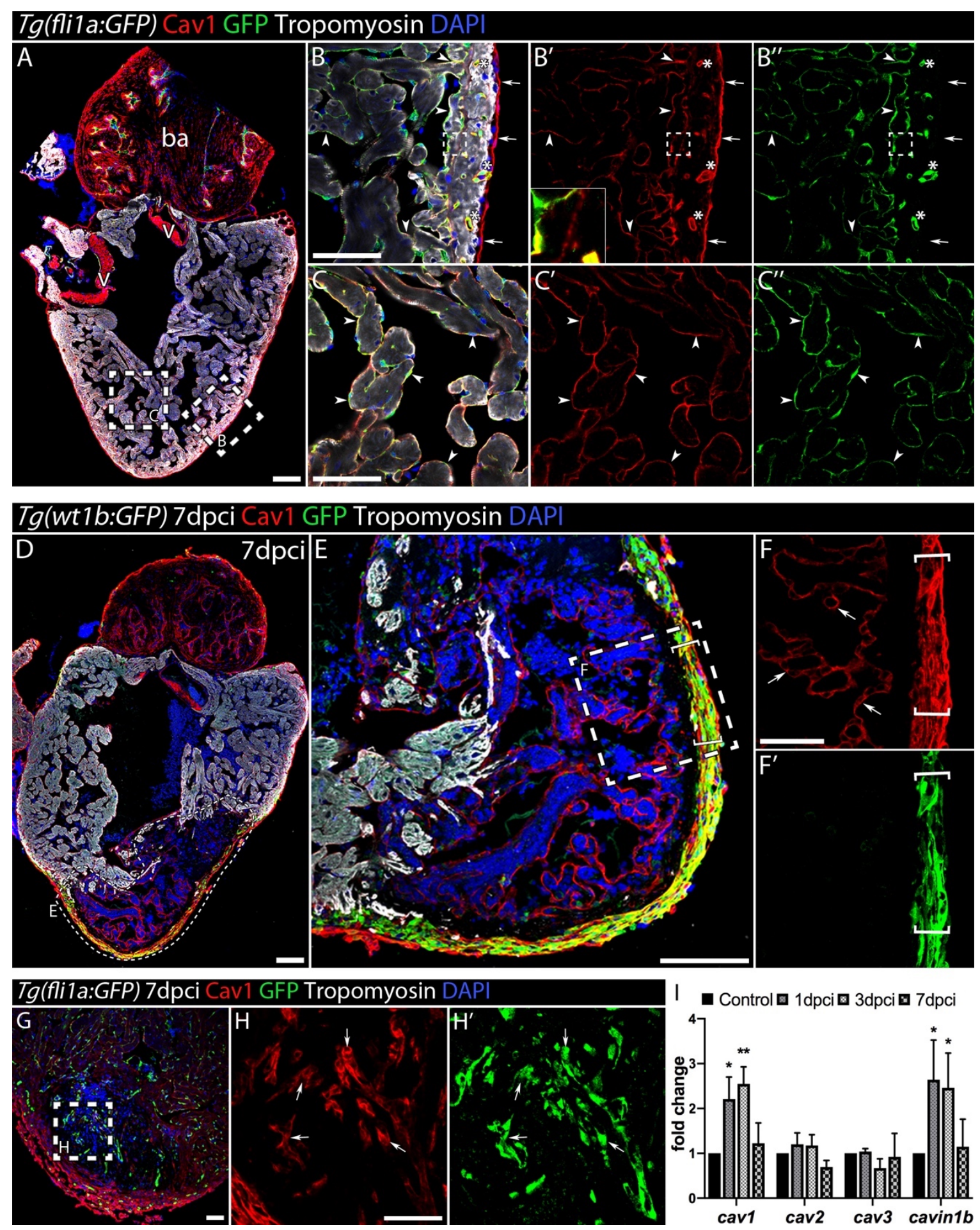

Figure 24. Expression analysis of Cav1 in the adult heart

(A-C") Immunofluorescence staining of Cav1 and Tropomyosin (CM) in control $\operatorname{Tg}$ (flila:GFP) heart. (B-C') Cav1 overlapped with GFP in the endothelium (asterisks), in the endocardium (arrowheads) and expressed in the epicardium (arrows). Cav1 was also detected in the bulbous arteriosus (ba), in the valves (v) and in the zone between the cortical and trabecular CM (insert in $\left.\mathrm{B}^{\prime}\right)$. (D-F') Cav1 immunostaining in 7dpci $\operatorname{Tg}(w t 1 b$ :GFP) injured heart. (D) Dashed area donates the injured area. (E) Magnification of the injured area from D. (F-F') Higher magnification of the dashed area in E, arrows indicate Cav1 in the endocardium and brackets in the epicardium. $\left(\mathrm{G}-\mathrm{H}^{\prime}\right)$ 
Immunolabeling of Cav1 in $7 \mathrm{dpci} \operatorname{Tg}(f l i l a: G F P)$ heart. $\left(\mathrm{H}-\mathrm{H}^{\prime}\right)$ Arrows indicate $\mathrm{Cav}^{+}{ }^{+}$endocardial cells. (I) qPCR analysis of caveolae-related genes during regeneration. Mean \pm S.D., BrownForsythe and Welch ANOVA tests, ${ }^{*} \mathrm{P}<0.05,{ }^{*} \mathrm{P}<0.01$. Scale bars $100 \mu \mathrm{m}$ in $\mathrm{A}, \mathrm{D}, \mathrm{E}$ and $50 \mu \mathrm{m}$ in the rest.

\section{Caveolin-2 and Cavin1 expression in control and injured adult hearts}

We further investigated the expression of two more proteins, Cav2 and Cavin1, so that we could have a better understanding of the expression pattern of caveolae-related proteins in the adult heart. From in vitro expression studies, CAV2 has been reported to follow the expression of CAV1 in different cell lines, so we examined if this was the case also in the zebrafish heart (Figure 25A$\mathrm{D}^{\prime \prime}$ ) (Mora et al., 1999). Cav2 immunolabelling showed expression in the endothelium, endocardium and epicardium in control and cryoinjured hearts, similarly to Cav1 (Figure 25A, C; asterisks in Figure 25B-B", arrowheads in Figure 25B-B", D and arrows in Figure 25B-B" and D-D", respectively). However, we did not detect Cav2 in the zone between cortical and trabecular CM. Cavin1 is also important for caveolae formation and stability (Lim et al., 2017; Liu et al., 2008), so we examined if its expression in the zebrafish heart was similar to the other caveolaerelated proteins (Figure 25E-J"). Cavin1 showed a similar distribution to Cav1, and was detected in the epicardium (Figure 25E; Figure 25F-F" arrows), endothelium (Figure 25F-F"' asterisks) and in-between the cortical and trabecular myocardium (Figure 25F-F" brackets), but not in the endocardium. Cavin1 was also weakly expressed in CM (Figure 25F-F" arrowheads). The epicardial and endothelial expression did not change after cryoinjury, where Cavin1 was strongly

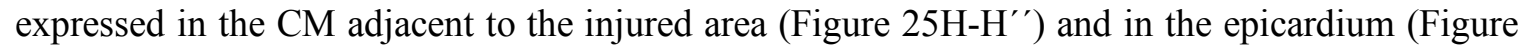
25I-J'). Overall, Cav2 followed Cav1 expression in the adult heart, whereas Cavin1 was not detected in the endocardium but in CM, and Cavin1 expression was elevated after injury in the proliferative $\mathrm{CM}$ in the border zone between healthy and damaged tissue.

\section{Generation of $\operatorname{cav1-KO}$ zebrafish by CRISPR/Cas9}

We decided to generate cav1 mutant zebrafish to study the role of Cav1 and caveolae during heart regeneration. The $\mathrm{C}$-terminal domain of the protein is important for Cav1 oligomerization, intercellular trafficking and for biogenesis of caveolae (Schlegel and Lisanti, 2000). Previous studies have shown that truncation of the C-terminal domain results in Cav1 arrested at the Golgi and blockage of caveolae formation. Interestingly, mutations in the C-terminal domain have been identified in human diseases and associated with pulmonary hypertension, atypical lipodystrophy and hypertriglyceridemia (Austin et al., 2012; Cao et al., 2008). Therefore, our initial strategy was to introduce mutations in the C-terminal tail so that we could block Cav1 intracellular trafficking and caveolae formation, without affecting the transcription of the cavl that may lead to genetic compensation (El-Brolosy et al., 2019). We have used the CRISPR/Cas9 gene editing 

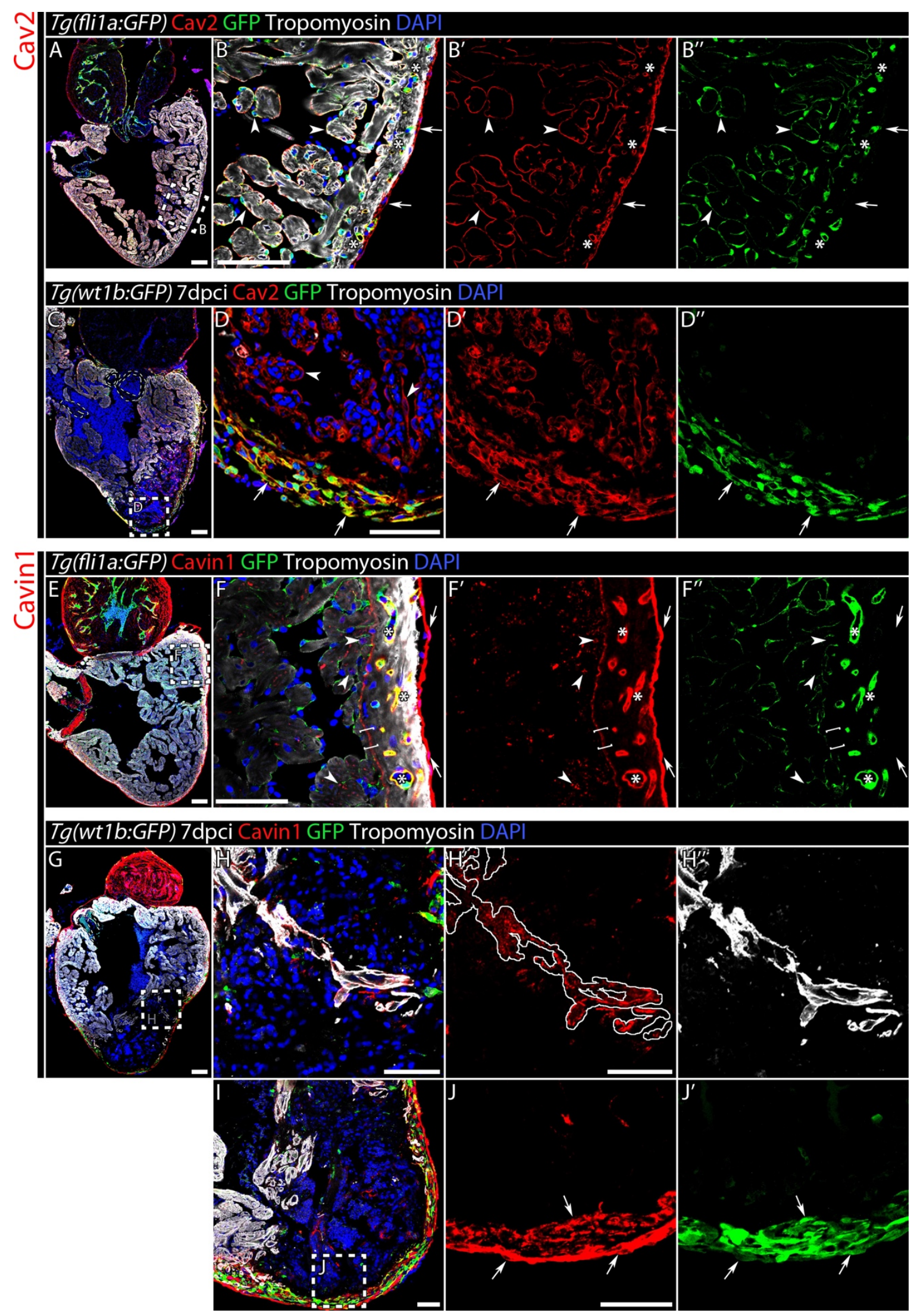

Figure 25. Caveolin-2 and Cavin 1 expression in control and regenerating heart

(A-B") Immunostaining of Cav2 in control $T g(f l i l a: G F P)$ heart. (B-B' ') Higher magnification of the dashed area in A, arrowheads indicate the endocardial, arrows the epicardial and asterisks the 
endothelial Cav2 expression. (C-D') Labelling of Cav2 in 7dpci $\operatorname{Tg}(w t 1 b: G F P)$ hearts. Dashed lines in $\mathrm{C}$ indicate the valves (D-D') Higher magnification of the dashed area in C. Arrows donate the epicardial expression of Cav2. (E-F") Cavin1 immunolabelling of in control $\operatorname{Tg}(f l i 1 a$ :GFP) heart. (F-F') Magnification of the dashed area in E. Cavin1 was found in the epicardium (arrows), endothelium (asterisks), between cortical and trabecular myocardium (brackets) and in CM (arrowheads). (G-J') Expression of Cavin1 in 7dpci $\mathrm{Tg}(w t 1 b: G F P)$ hearts. Dashed lines in $\mathrm{G}$ indicate the valves. $\left(\mathrm{H}-\mathrm{H}^{\prime \prime}\right)$ Magnification of the area in $\mathrm{G}$. $\left(\mathrm{H}^{\prime}\right)$ white line marks the periphery of the $\mathrm{CM}$ in $\mathrm{H}^{\prime \prime}$. (I) Magnification of the injured area from $\mathrm{G}\left(\mathrm{J}-\mathrm{J}^{\prime}\right)$ Higher magnification of the area in I. Arrows indicate Cavin $1^{+}$epicardial cells. Scale bars A, C, E, G $100 \mu \mathrm{m}$ and $50 \mu \mathrm{m}$ in the rest.

technology to target the third exon of cavl, that encodes the Cav1 carboxy-terminus (Figure 26). cavl is a short gene that generates two transcripts, cavla and cavlb, sharing the majority of their coding sequences, including the last exon (Figure 26A) (Fang et al., 2006). We analysed the third exon to identify the best target site but none appropriate target was found. Consequently, we selected the fittest target site leading to less potentially intronic off-targets, although this was in the region that translates the second a-helix of the intermembrane domain. After microinjection of the gene editing reagents in the fertilized egg and genomic screening in embryos, we identified mutations in the cavl locus, being predominantly deletions (Figure 26B). The predicted effect in the protein was an ORF shift that would lead to an amino acid change and a premature stop codon (Figure 26C). The identified mutations were L114fsX135, L114fsX136, A115fsX166 and L114_A115del, refer to as X135, X136, X166 and 115del, respectively. All mutant larvae had no apparent morphological abnormalities, developed normally and were fertile. The alteration in Cav1 structure directly impacts in caveolae formation, so we examined by in silico 3D modelling the ability of Cav1 mutants to form homodimers or heterodimers (with WT Cav1), and entering the plasma membrane (Sup. Figure 7). The analysis predicted that X136 and X166 mutant versions would fail to form homo-, or heterodimers, or that they would be unstable without being able to enter in the membrane, whereas X135 and 115del homodimers were predicted to be inserted into the membrane, with $115 \mathrm{del}$ resembling the WT Cav1. We further analysed the mutants by qPCR excluding $115 \mathrm{del}$, since it behaved similarly to the WT in the in silico analysis (Figure 26D). X134 and X135 had similar effects in caveolae-related genes, decreasing significantly cavl transcription, possible through non-sense mediated decay (Frischmeyer et al., 2002) with a parallel increase of cav2. Interestingly, the reduction in cavl expression was not observed in the X166 embryos although cav2 was upregulated. No transcriptional deregulation was detected for cav3, cavin1b or cavy (Kirkham et al., 2008) for all three genotypes. The effects in cavl transcript was confirmed by WM-ISH (Figure 26E, F'), which revealed that cavl expression was decreased in X136 embryos, while in X166 was similar to WT. 

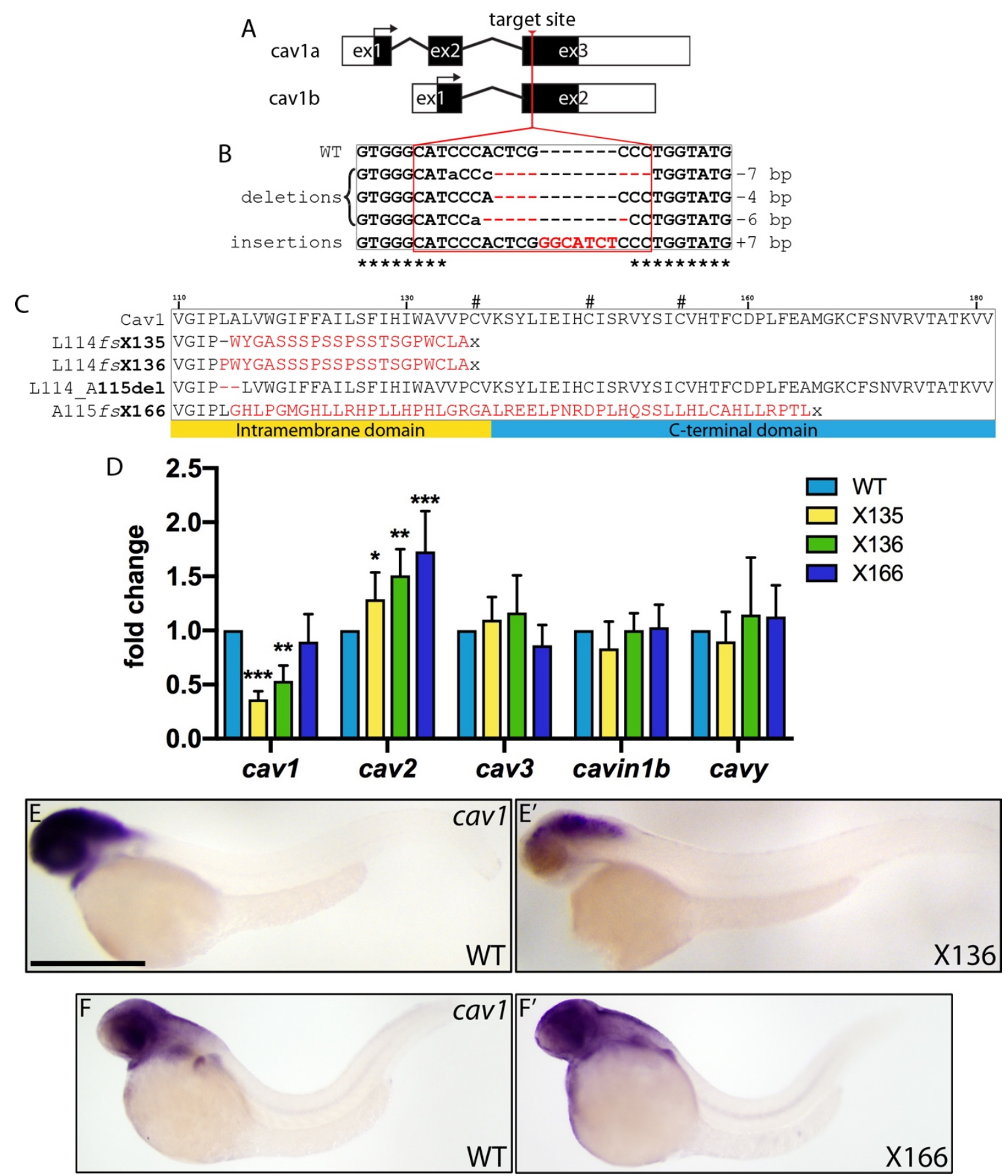

Figure 26. Generation of cav1-KO by CRISPR/Cas9 and transcriptional analysis

(A) Schematic representations of cavl locus with the two transcripts, cavla and cavlb. Target site of the guide RNA in red arrowhead. ex = exon. (B) Identified genetic mutations. Red hyphens indicate deletions, red uppercase letters insertions and black lowercase letters silent mutations. bp = base pairs. (C) Cav1 domain organization and the predicted effect on the protein. The novel amino acids are in red, hashtags showing the conserved cysteines and $\mathrm{x}$ indicates a stop codon. (D) qPCR analysis of 2 dpf embryos for the caveolae-related genes. Mean \pm S.D., t-test, $* \mathrm{P}<0.05, * * \mathrm{P}<0.01$, *** $\mathrm{P}<0.001$. ( $\left.\mathrm{E}-\mathrm{F}^{\prime}\right) \mathrm{WM}-\mathrm{ISH}$ of cav1 in WT controls (E and F), X136 (E') and X166 (F') 2dpf embryos. Scale bar $200 \mu \mathrm{m}$. 


\section{Analysis of Cav1a and Cav1b expression in X136 and X166 hearts}

As described above, the mutant X136 and X166 Cav1 versions were predicted neither to homodimerize, nor to be embedded in the membrane, and X135 had decreased transcriptional expression. Therefore, we investigated the Cav1 protein distribution by immunofluorescent. We labelled 7dpci WT, X136 and X166 hearts with an antibody that recognizes specifically Cav1a (Figure 27). Surprisingly, Cav la was completely lost in the adult hearts in both X136 (Figure 27DF) and X166 (Figure 27G-I). The normal Cav1a signal in the epicardium, endocardium, endothelium, valves and bulbus arteriosus (Figure 27 A-C) was absent in X136 and X166 hearts (Figure 27 D-I). We then labelled hearts with an antibody that recognises both Cav1 proteins, Cav1a and Cav1b (Figure 28). In X136 and X166, Cav1b (since we have lost Cav1a expression) was absent from the epicardium, endocardium, bulbus arteriosus and valves, similarly to Cav1a (Figure 28DI). Similar results were obtained with X135 hearts, in contrast to $115 \mathrm{del}$, in which Cav1 was normally expressed (Sup. Figure 8). However, some signal was detected using the Cav1 antibody in CM that was not noticed in the WT hearts, possibly due to the strong Cav1 expression in endocardium and coronary endothelial cells. To clarify if the signal in the CM was specific for Cav1, and not an artefact or unspecific labelling, we performed WB analysis in control and injured hearts (Figure 29A). WB using the Cav1a specific antibody revealed that X136 and X166 hearts have lost expression of Cavla. However, WB against both Cav1, revealed three bands in control hearts, instead of the expected two, Cav1a and Cav1b bands. In X136 and X166 hearts, the two bands corresponding to Cav1 a and b were absent (Figure 29A). Additionally, no truncated Cav1a or Cav1b were detected, since the mutated versions of Cav1 were predicted to have a lower molecular weight than the Cav1b band. To ensure that we have inactivated the expression of both Cav1 proteins, we analysed by immunostaining and WB a Cav1a-KO (hereafter X21) fish that we have generated (Sup. Figure 9). WB analysis revealed that in X21 hearts, Cav1a was lost in contrast to Cav1b, although the third band was still detectable (Figure 29B). We also observed lack of expression of both Cav1 isoforms in fins, in which the third band was absent (Figure 29C), suggesting that this band and the signal in CM were not Cav1-specific. These results indicate that the X136 and X166 genetic mutations that were predicted to lead to expression of a shorter Cav1 protein, cause the complete loss of Cav1 expression in the adult heart. Hence, both X136 and X166 genetic mutations led to cavl knockout that for simplicity we will keep referring to as X136 and X166, respectively. 


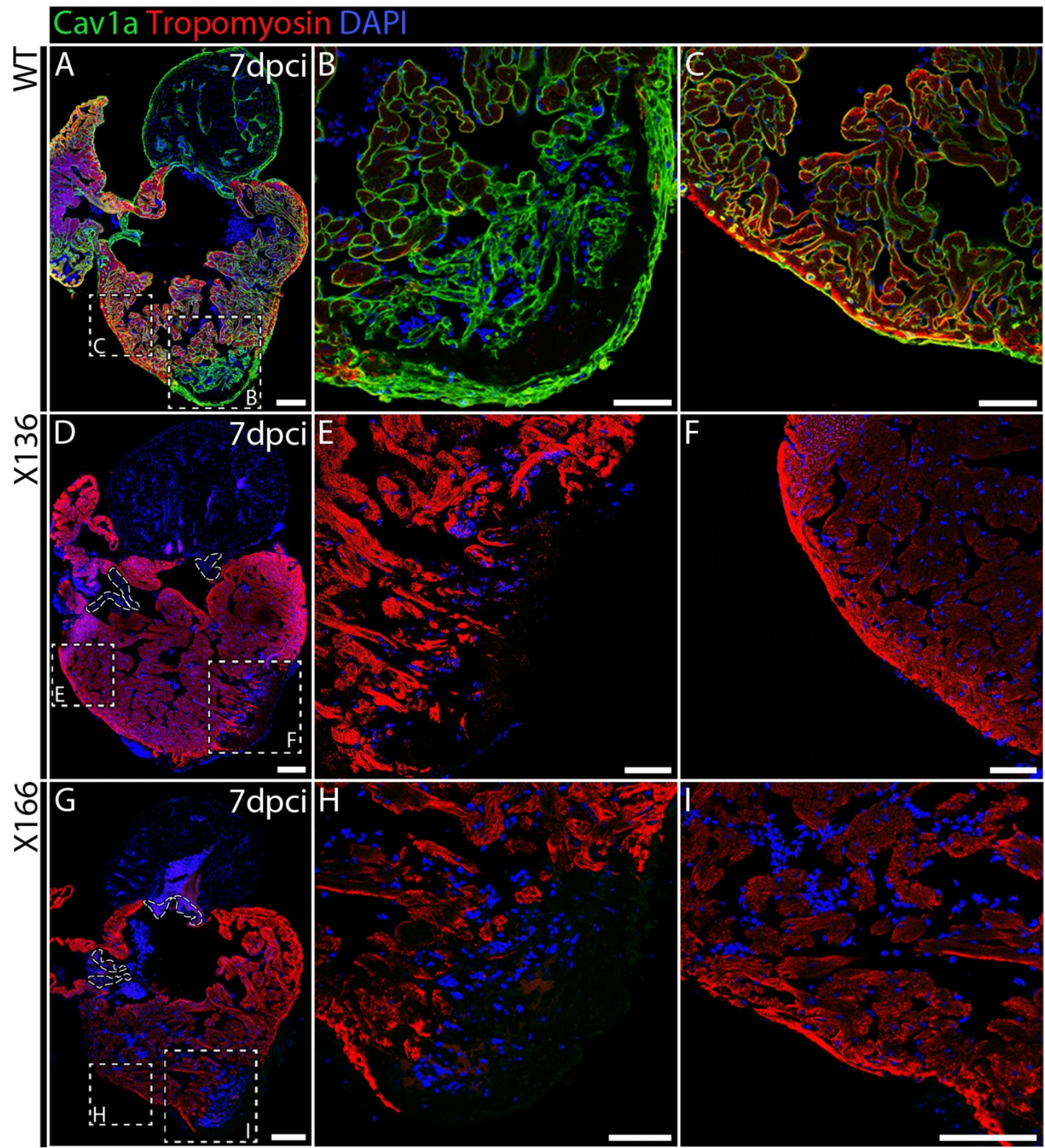

Figure 27. Expression analysis of Cav1a in X136 and X166 hearts

(A-I) Immunostaining of Cav1a in 7dpci WT (A-C), X136 (D-F) and X166 (G-I) in hearts. B and $\mathrm{C}$; E and F; H and I, magnifications of the selected areas in A, D and G, respectively. (D and G) Outlines of the valves in dashed line. Scale bars $100 \mu \mathrm{m}$ in A, D, G; $50 \mu \mathrm{m}$ in the rest. 


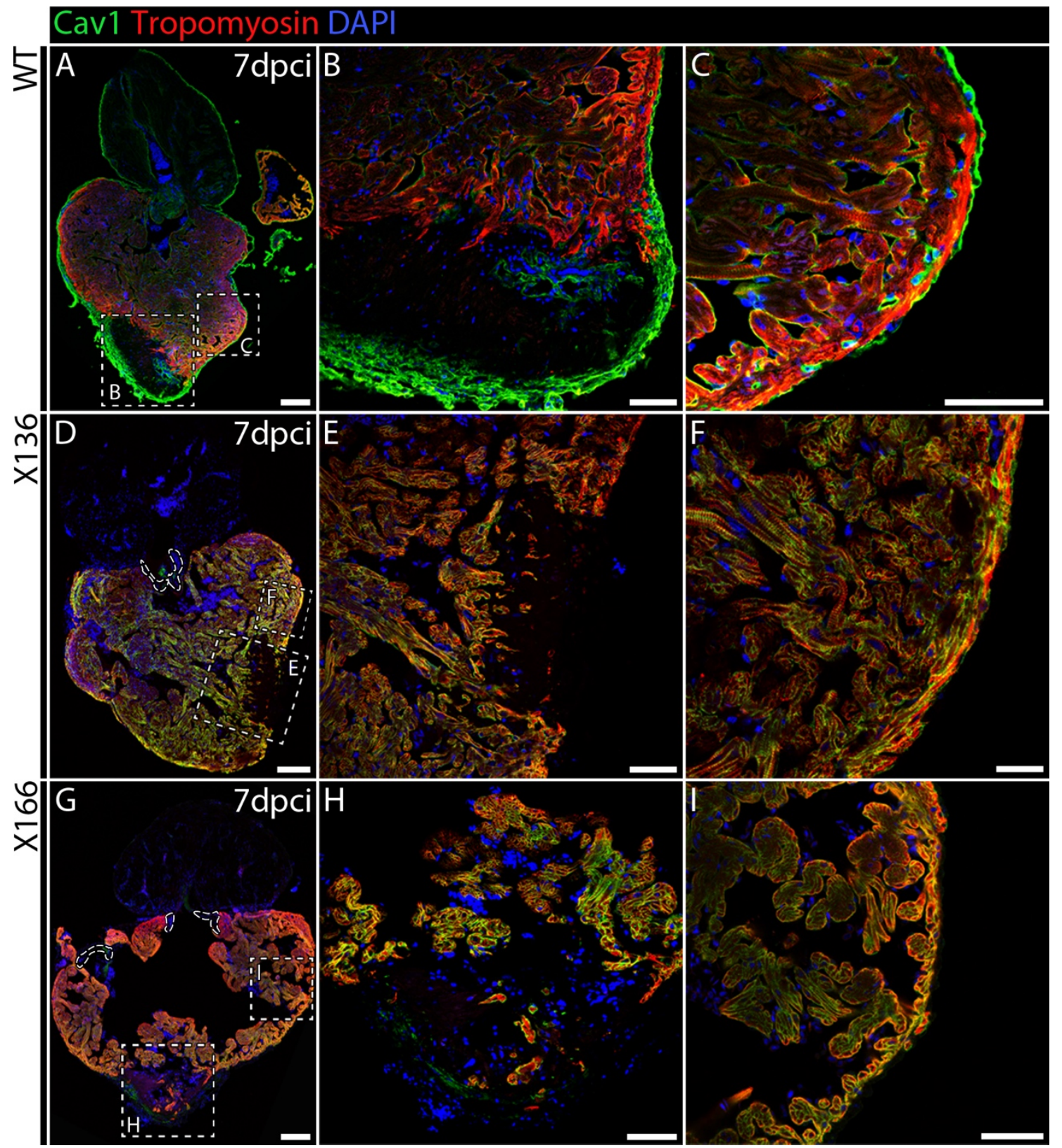

Figure 28. Expression analysis of Cav1b in X136 and X166 hearts

(A-I) Immunostaining of Cav1 in 7dpci WT (A-C), X136 (D-F) and X166 (G-I) hearts. B and C, E and F, H and I, magnifications of the selected areas in A, D and G, respectively. (D and G) Outlines of the valves in dashed line. Scale bars $100 \mu \mathrm{m}$ in A, D, G; $50 \mu \mathrm{m}$ in the rest. 


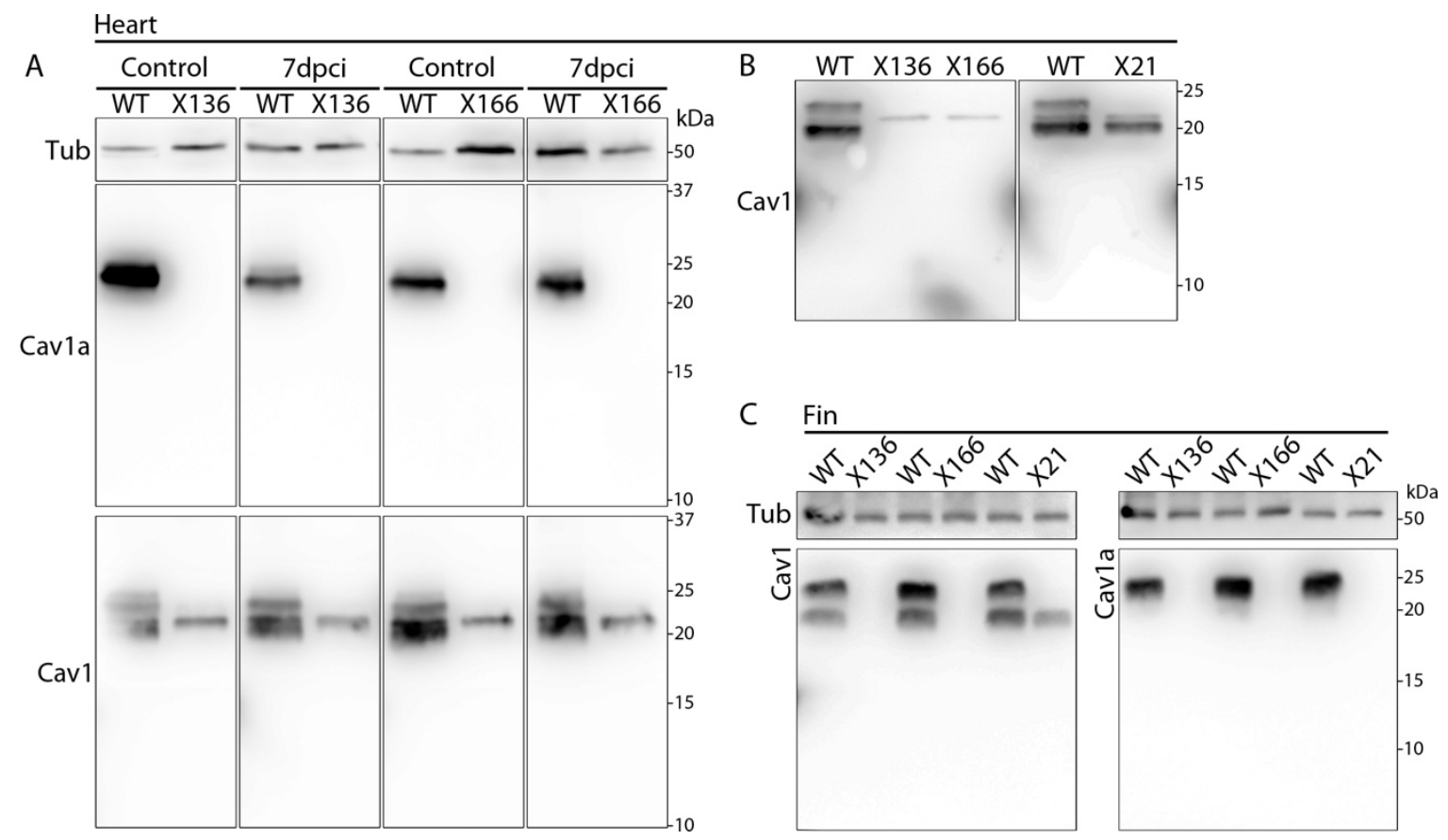

Figure 29. WB analysis of Cav1 in X136 and X166 hearts

(A) Control and 7dpci WT, X136 and X166 hearts were analysed by WB using antibodies against Cav1a or Cav1 (Cavla and Cav1b). Note the absent of Cav1 truncations below 20kDa. Cav1a $\approx$ $22-24 \mathrm{kDa}, \mathrm{Cav} 1 \mathrm{~b} \approx 20-21 \mathrm{kDa}, \mathrm{X136a} \approx 15.16 \mathrm{kDa}, \mathrm{X136b} \approx 11.35 \mathrm{kDa}, \mathrm{X} 166 \mathrm{a} \approx 18.79 \mathrm{kDa}$, $\mathrm{X} 166 \mathrm{~b} \approx 14.98 \mathrm{kDa}$. (B) WB analysis in control hearts of WT, X136, X166 and X21 animals. (C) Fin samples from all genotypes analysed by WB. Tub $=$ Tubulin, $\mathrm{kDa}=$ kilodalton.

\section{Cavin1b expression in the X136 and X166 hearts}

Cav1 and Cavin1 are essential components of caveolae (Hill et al., 2008) and deletion of Cav1 decreases Cavin1 expression in mice (Hansen et al., 2013). Therefore, we asked if Cavin1 was affected by Cav1 loss in our animals (Figure 30). In the WT heart, Cavin1 had a strong expression in the epicardium, in CM next to the damaged tissue, in the bulbus arteriosus and in the valves (Figure 30A-D' and I-L'). In the cavl-KO hearts, Cavin1 expression was decreased (Figure 30E-H' and M-P'). Specifically, in the valves Cavin1 was absent (Figure 30E and M), whereas it had decreased expression in the bulbus arteriosus (Figure 30E and $\mathrm{M}$ ) and in the CM within the proliferative zone (Figure 30G-G' and $\mathrm{O}^{\prime} \mathrm{O}^{\prime}$ ). The decreased Cavin 1 protein expression was also observed by WB analysis (Sup. Figure 10). 

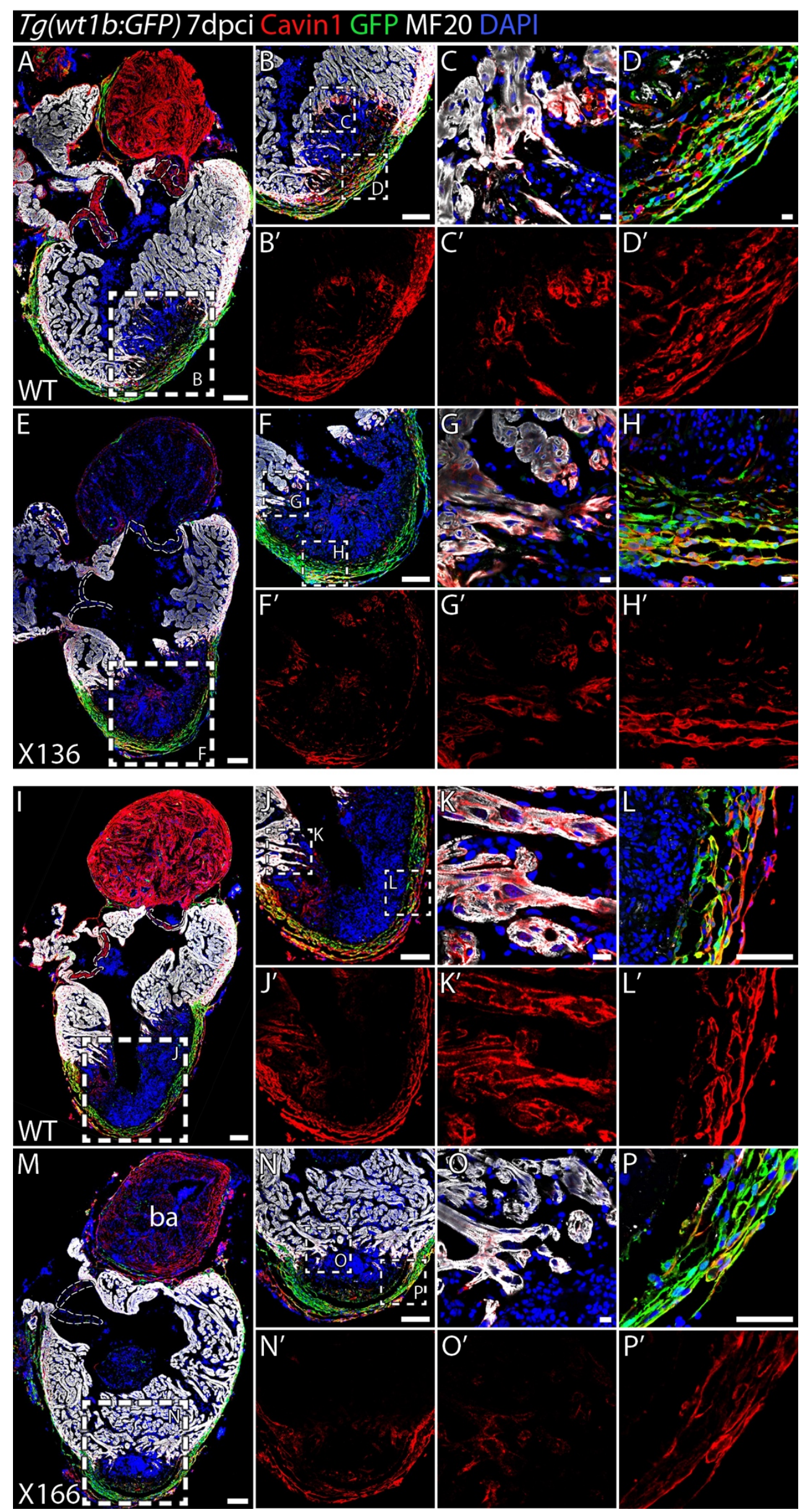


\section{Figure 30. Cavin1b expression in the X136 and X166 hearts}

(A-H') Immunostaining of Cavin1 in 7dpci WT and X136 $\mathrm{Tg}(w t 1 b: G F P)$ hearts. (B) Magnification of selected area in A. (C-D') Magnifications of the dashed areas in B. (F) Magnification of selected area in E. (G-H') Magnifications of the dashed areas in F. (I-P') Immunostaining for Cavin1 in 7dpci WT and X166 $\mathrm{Tg}(w t 1 b: G F P)$ hearts. (J) Magnification of selected area in I. (K-L') Magnifications of the areas marked in J. (N) Magnification of selected area in M. (O-P') Magnifications of the dashed areas in N. (A, E, I, M) Dashed lines mark the valves. Scale bars, A, $\mathrm{B}, \mathrm{E}, \mathrm{F}, \mathrm{I}, \mathrm{J}, \mathrm{M}$ and $\mathrm{N} 100 \mu \mathrm{m} ; 50 \mu \mathrm{m}$ in the rest.

\section{Loss of caveolae in the X136 and X166 hearts}

Expression of Cavl in a system without endogenous expression leads to de-novo caveolae formation (Fra et al., 1995), whereas deletion of Cav1 results in loss of caveolae (Drab et al., 2001; Razani et al., 2001a; Zhao et al., 2002). Hence, we asked if our cav1-KO zebrafish were able to form caveolae. To answer this question, we analysed WT, X136 and X166 hearts by transmission electron microscopy (TEM). We found caveolae in abundance in WT hearts (Figure 31A). The endothelial cells of the coronary vasculature were filled with membrane-bound caveolae, as well as with numerous intracellular caveola-like vesicles (Figure 31B, arrowheads). Additionally, the plasma membrane of epicardial cells was also densely packed with caveola-invaginations, distributed equally in the apical or basal surface (Figure 31C, arrowheads). To quantify the caveolae, we focused on the endothelial cells of the coronary vasculature (Figure 32). Likewise, WT control hearts were filled with caveolae (Figure 32A', D' arrowheads and Figure 32 A' insert). In contrast, X136 and X166 hearts were characterized by the lack of caveolae (Figure 32B-C', D$F^{\prime}$ ). Neither membrane-bound caveolae nor intracellular caveola-like vesicles were found, demonstrating the complete loss of caveolae in X136 and X166 hearts. 


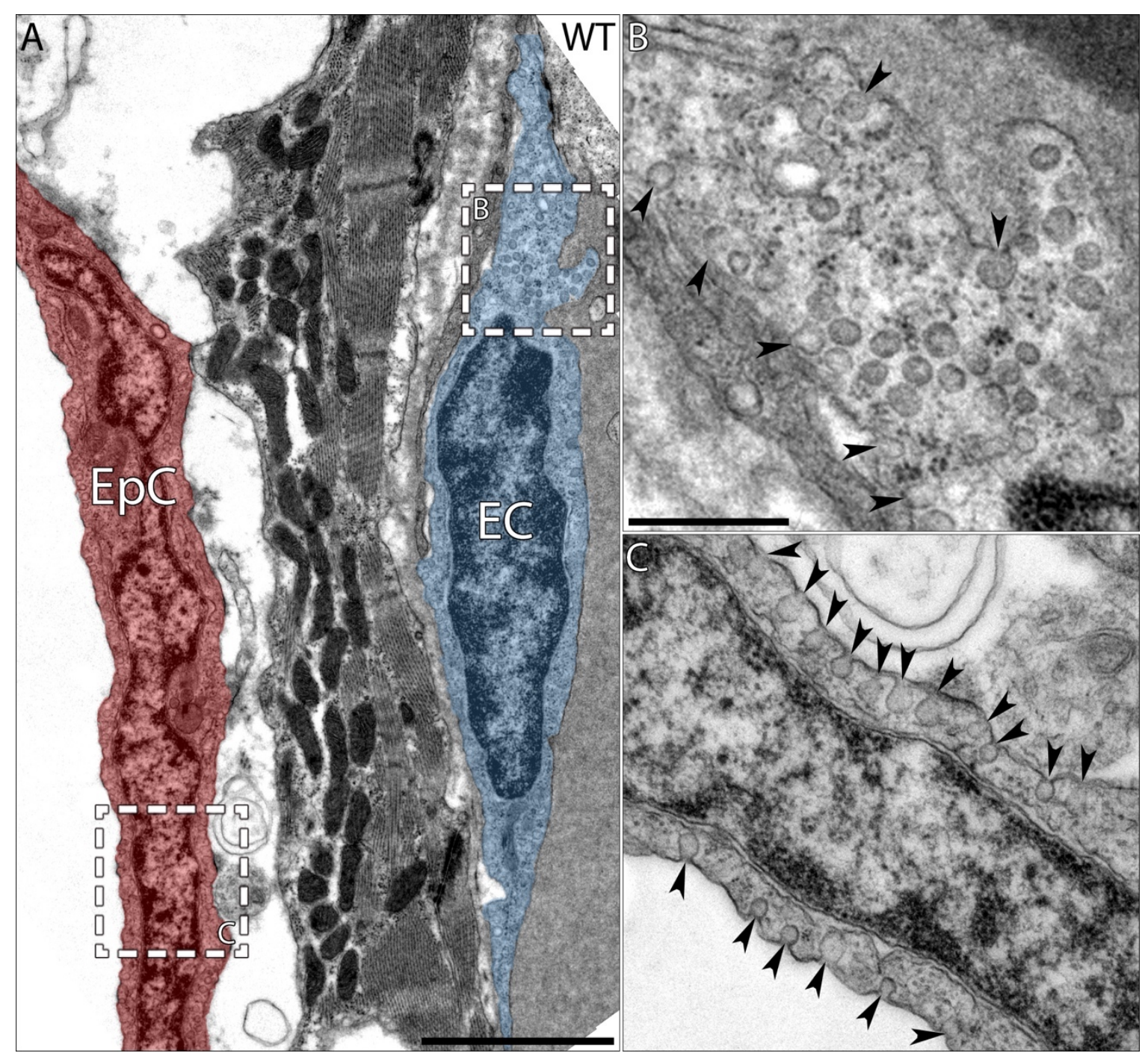

Figure 31. Caveolae abundance in adult heart

(A) Pseudo-colorized TEM images of an epicardial cell (EpC, red) with an underlined endothelial cell (EC, blue) of the cortical layer. (B, C) Higher magnifications of the selected areas in A, arrowheads indicate membrane-bound caveolae. Scale bars, A 200nm, B and C 50nm. 
Results - Caveolin-1
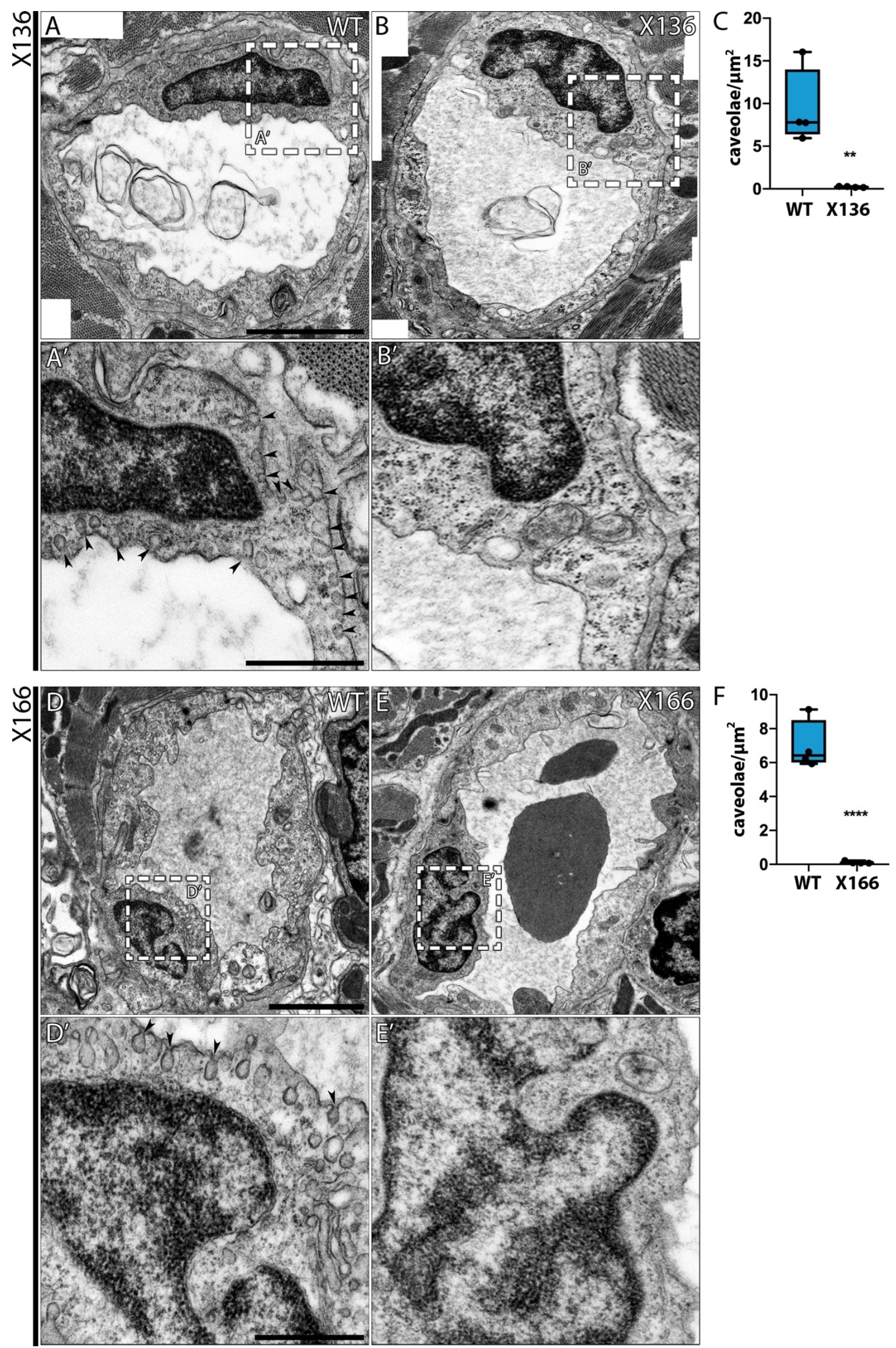


\section{Figure 32. Loss of caveolae in X136 and X166 hearts}

(A-B', D-E') TEM images of WT control (A-A', D-D'), X136 (B-B') and X166 (E-E') endothelial cells from the coronary vasculature for caveolae visualization. ( $\mathrm{A}^{\prime}$ and $\mathrm{D}^{\prime}$ ) Arrowheads indicate membrane-bound caveolae. Scale bars, A-B, D-E $1 \mu \mathrm{m}, \mathrm{A}^{\prime}-\mathrm{B}^{\prime}, \mathrm{D}^{\prime}-\mathrm{E}^{\prime} 0,5 \mu \mathrm{m}$. (C and F) Quantification of the caveolae number per $\mu \mathrm{m}^{2}$ of endothelial cell. $\mathrm{n}_{\mathrm{WT}}=\mathrm{n}_{\mathrm{X} 136}=\mathrm{n}_{\mathrm{X} 166}=4$, t-test, $* * \mathrm{P}<0.01, * * * * \mathrm{P}<0.0001$.

\section{Response of caveolae-depleted hearts to injury}

We found caveolae and Cav1 in different cell types of the zebrafish heart, thus we examined the effect that loss of Cav1/caveolae may have in heart regeneration. We cryoinjured WT and X136 hearts and allow them to regenerate for 90-days (Chablais and Jaźwińska, 2012; Gonzalez-Rosa et al., 2011; Schnabel et al., 2011). We then analysed the hearts by Acid Fuchsin Orange-G (AFOG) staining that stains myocardium in orange and collagen in blue, and helps to distinguish the damaged area from the healthy myocardium. We found that X136 hearts regenerated similarly to the WT hearts at 90dpci (Figure 33G-J). Therefore, we decided to monitor the regeneration process by analysing injured heart 30-, and 60dpci (Figure 33A-F). The X136 hearts had similar scar size than WT controls at $30 \mathrm{dpci}$ and $60 \mathrm{dpci}$. These results were surprising, since heart regeneration has been reported to be impaired after ventricular resection in a zebrafish cav1$K O$ model (Cao et al., 2016). Therefore, we also examined X166 animals, in which cavl was not affected transcriptionally. We cryoinjured WT and X166 hearts and followed the regeneration process every 30-days (Figure 34). Similarly, X166 hearts regenerated normally and we did not detect any differences in the size of the injury between WT and X166 hearts at 30-, 60- or 90dpci. In the CAV1-KO mouse, cryoinjury leads to extensive collagen deposition and cardiac remodelling (Miyasato et al., 2011) therefore we took advantage of the AFOG staining to examine two more parameters, the collagen and fibrin in the injury, and the volume of the ventricle (Sup. Figure 11). However, no differences were detected between WT and cavl-KO hearts, neither in collagen deposition or fibrin amount in the injury, nor in the size of the ventricle. Overall, these results indicate that loss of caveolae does not affect neither the formation or degradation of the scar tissue nor heart regeneration. 

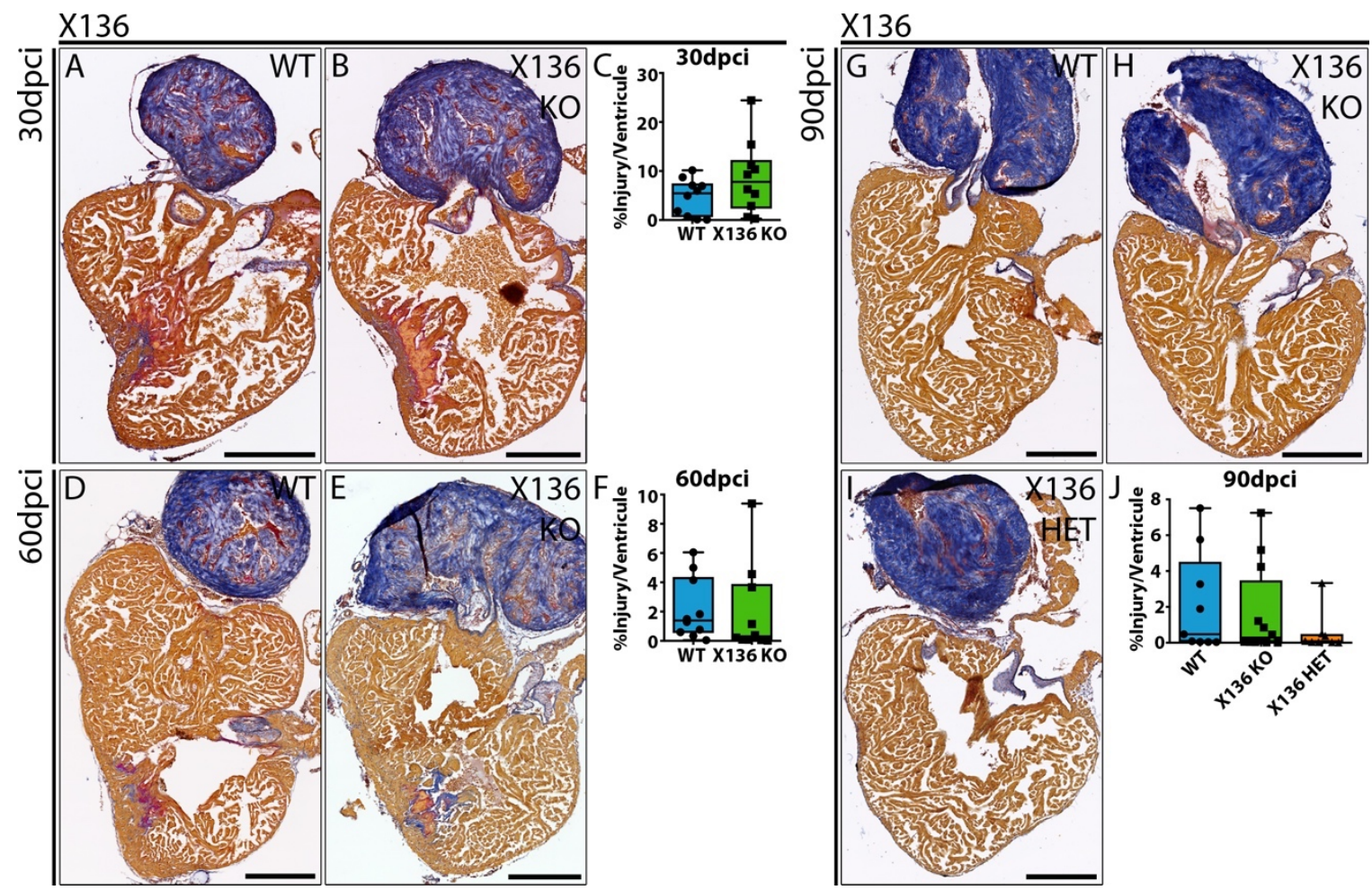

Figure 33. Monitoring of the regeneration process in X136 hearts

(A-J) WT and X136 hearts were cryoinjured and harvested 30- (A-C), 60- (D-F), or 90dpci (G-J), and processed for AFOG staining that labels collagen in blue, fibrin in red and the healthy myocardium in brown. (C, F, J) The size of the injuries was quantified as percentage of the damaged tissue (collagen and fibrin) to the total area of the ventricle. (C) 30 dpci, $n_{\mathrm{WT}}=\mathrm{n}_{\mathrm{X} 136}=10$. (F) 60dpci, $\mathrm{n}_{\mathrm{WT}}=\mathrm{n}_{\mathrm{X} 136}=10 .(\mathrm{J}) 90 \mathrm{dpci}, \mathrm{n}_{\mathrm{WT}}=9, \mathrm{n}_{\mathrm{X} 136}=12$. t-test. Scale bars $250 \mu \mathrm{m}$. 


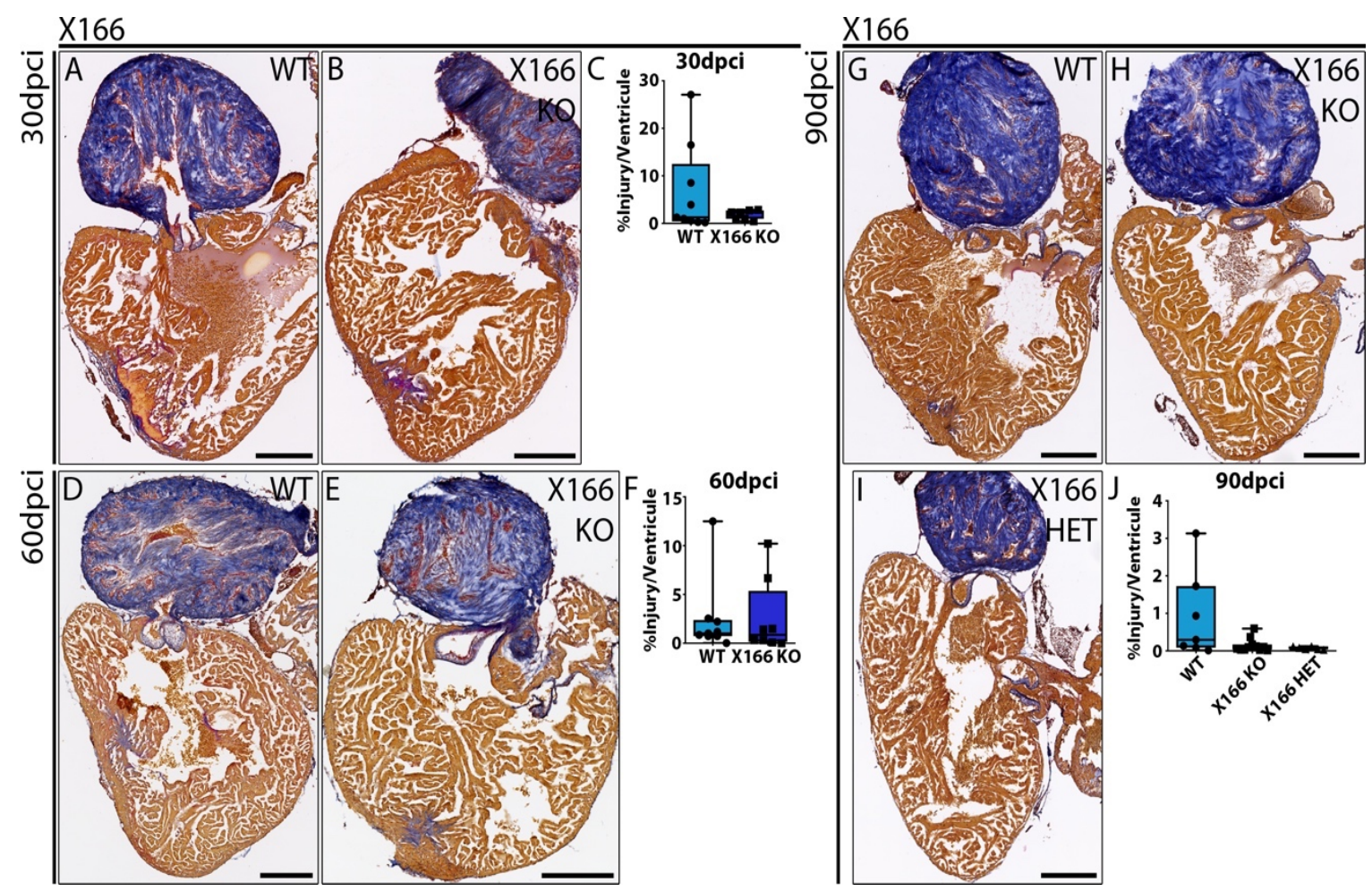

Figure 34. Investigation of X166 hearts regeneration

(A-J) AFOG staining of WT and X166 hearts 30- (A-C), 60- (D-F) and 90dpci (G-J). Collagen in blue, fibrin in red and healthy myocardium in brown. (C, F, J) The damaged area was quantified as the percentage of the collagen/fibrin area to the total ventricular area. (C) $30 \mathrm{dpci}, \mathrm{n}_{\mathrm{WT}}=9, \mathrm{n}_{\mathrm{X} 166}=$ 7. (F) $60 \mathrm{dpci}, \mathrm{n}_{\mathrm{WT}}=8, \mathrm{n}_{\mathrm{X} 166}=7$. (J) $90 \mathrm{dpci}, \mathrm{n}_{\mathrm{WT}}=7, \mathrm{n}_{\mathrm{X} 166}=11$. t-test. Scale bars $250 \mu \mathrm{m}$.

\section{TGF $\beta$ signalling activation is unaffected in X136 hearts}

Caveolae are involved via Cav1 in the regulation of the TGF $\beta$ pathway (Razani et al., 2001b), a major signalling cascade that controls ECM deposition and is active during zebrafish heart regeneration (Chablais and Jazwinska, 2012). Since both X136 and X166 cavl-KO hearts regenerated normally, we focused only in X136. To address TGF $\beta$ activity in the X136 hearts upon injury, we quantified the nuclear localization of phospho-Smad3 (psmad3), a downstream effector of TGF 3 (Figure 35). We used 14dpci $\operatorname{Tg}\left(f l i 1\right.$ : GFP) hearts to calculate the psmad3 ${ }^{+}$nuclei of the

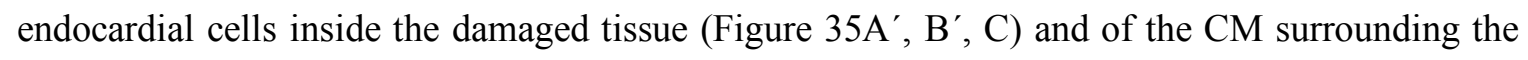
injured site (Figure 35A", B", D). The analysis revealed that TGF $\beta$ signalling was evenly active in WT and X136 hearts in both CM and in endocardial cells. Additionally, we examined if fibrotic deposition in intact X136 hearts was increased, since hearts of CAV1-KO mice show increased interstitial fibrosis (Cohen et al., 2003; Drab et al., 2001; Murata et al., 2007; Park et al., 2003). Therefore, we stained with Picrosirius red that labels collagen fibers, but we did not find a difference in interstitial fibrosis between WT and X136 hearts (Sup. Figure 12). Hence, loss of caveolae does not affect TGF $\beta$ activity in control or cryoinjured hearts. 

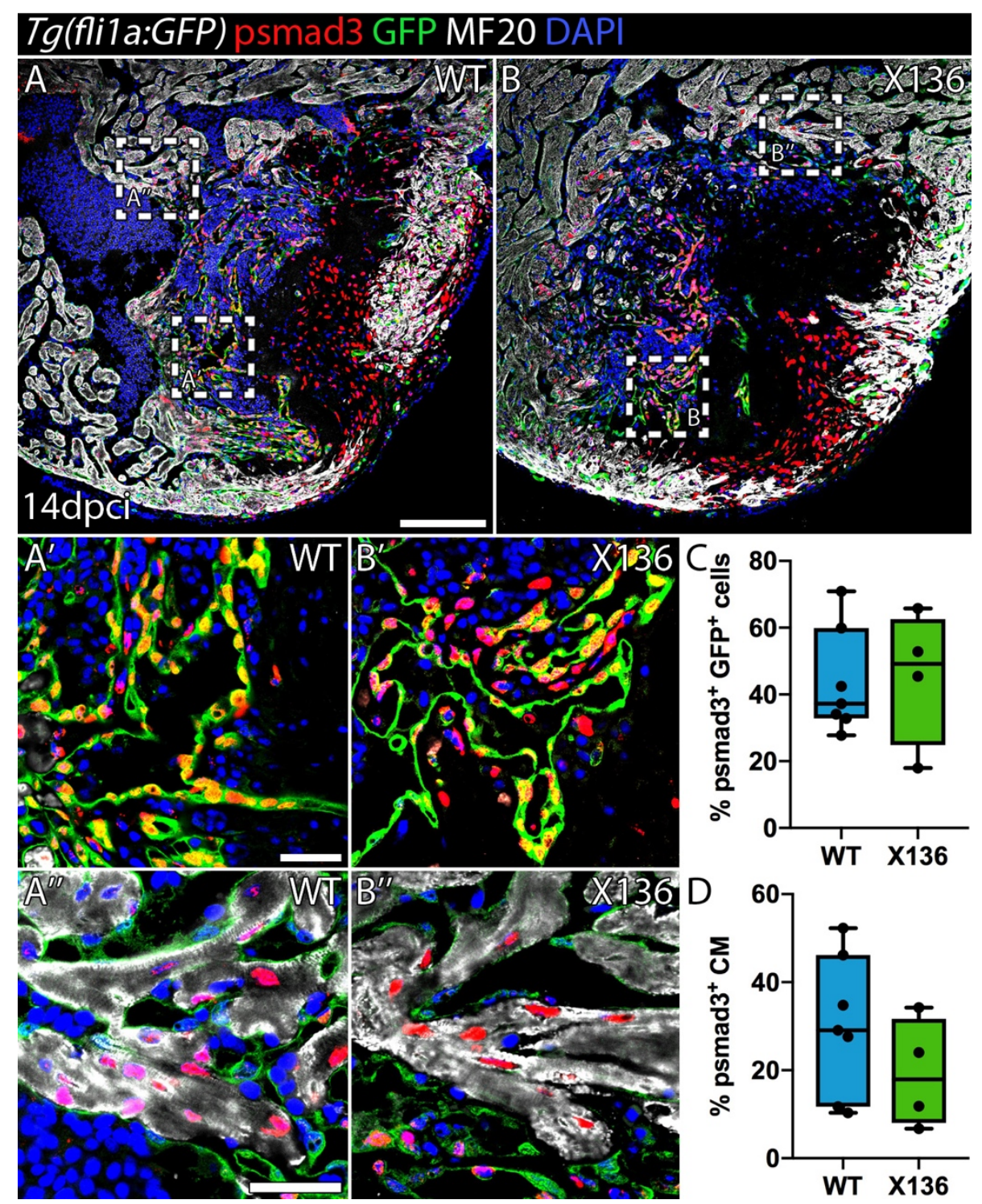

Figure 35. TGF $\beta$ signalling activation in injured caveolae-less heart

(A-D) 14dpci WT and X136 Tg(flila:GFP) hearts labelled for psmad3, GFP and MF20. (A', B') Magnification of $\mathrm{GFP}^{+}$endocardial cells marked in A, B. (B'-B') Higher magnifications of CM marked in A, B. (C) Quantification of the $\mathrm{psmad}^{+} / \mathrm{GFP}^{+}$in the injured area. t-test. $\mathrm{n}_{\mathrm{WT}}=7, \mathrm{n}_{\mathrm{X} 136}=$ 4. (D) Percentage of $\mathrm{CM}$ with psmad $3^{+}$nuclei in a $100 \mu \mathrm{m}$ area surrounding the damaged tissue. $\mathrm{t}-$ test, $\mathrm{n}_{\mathrm{WT}}=7, \mathrm{n}_{\mathrm{X} 136}=4$. Scale bars, $100 \mu \mathrm{m} \mathrm{A}$ and $\mathrm{B}, 25 \mu \mathrm{m}$ in the rest.

\section{Epicardial and endocardial cell function in X136 heart is unaffected}

We then examined the epicardial and endocardial cells in which Cav1 expression is high at $7 \mathrm{dpci}$ (Figure 36). We bred the $\mathrm{Tg}(w t 1 b$ :GFP) line with our cav1-KO backgrounds to examine epicardial proliferation by measuring the ratio between $\mathrm{BrdU}^{+}$and $\mathrm{GFP}^{+}$nuclei. We found no difference in epicardial proliferation between the WT and X136 hearts (Figure 36A-C). We then investigated the abundance of the endocardial cells within the damaged tissue, by breeding the double transgenic fish $\operatorname{Tg}(f l i l a: G F P) / \operatorname{Tg}(m y l 7: m R F P)$ that expressed GFP in endocardial/ 
endothelial cells and RFP in the membrane of CM with our cav1-KO backgrounds (Figure 36G-F). 3D volume rendering (Video 11, 12) and analysis of the $\mathrm{GFP}^{+}$cells inside the $\mathrm{RFP}^{-}$area showed that endocardial cells in the X136 hearts populated the damaged tissue similarly to the WT. These data indicate that epicardial proliferation as well as migration and proliferation of the endocardial cells is not affected in X136 hearts. Thus, loss of Cav1 and caveolae did not affect the function of epicardial or endocardial cells in heart regeneration.
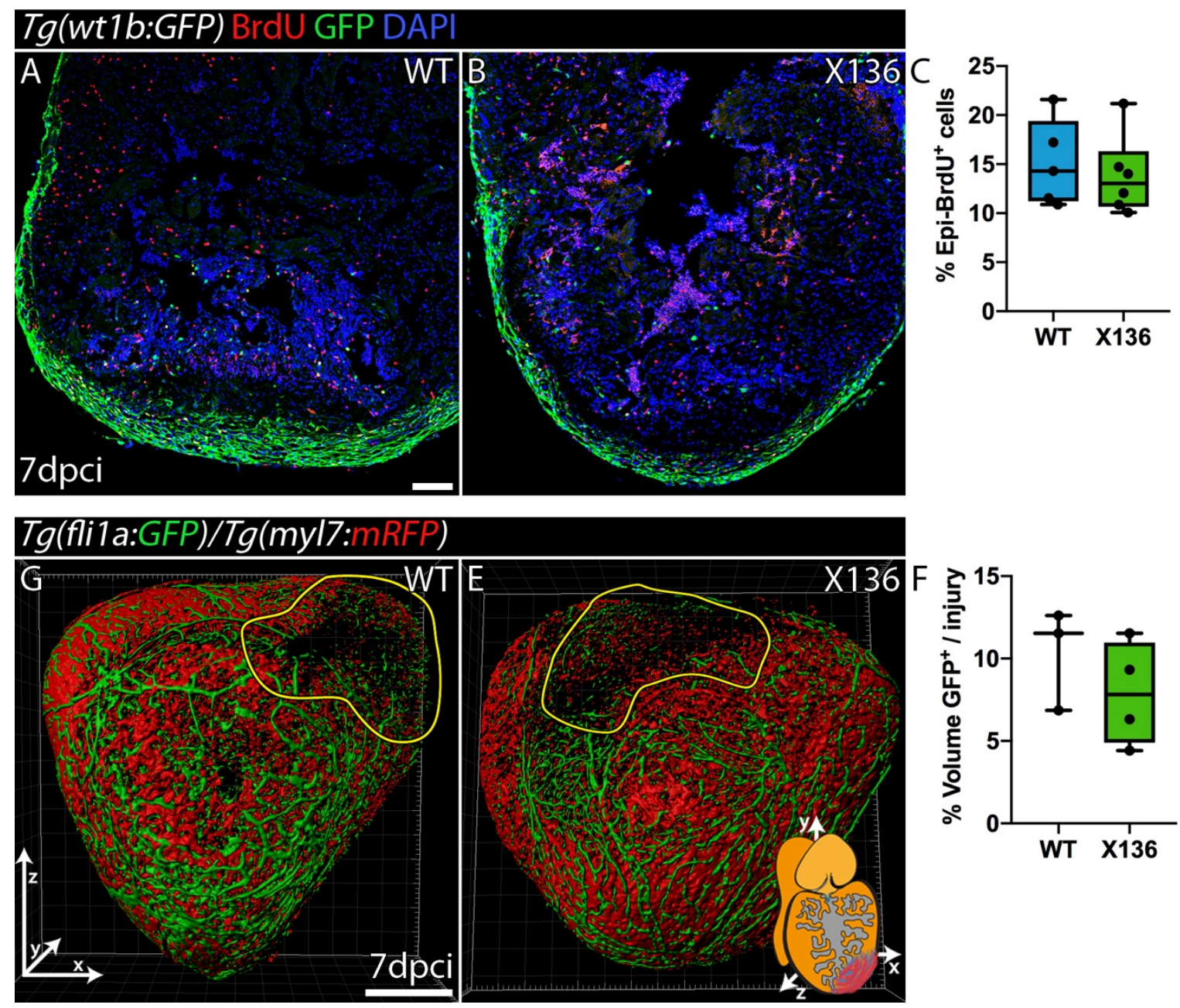

Figure 36. Epicardial proliferation and endocardial behaviour in X136 hearts after injury (A-B) Immunostaining of $7 \mathrm{dpci} \operatorname{Tg}(w t 1 \mathrm{~b}$ :GFP) heart sections labelled for BrdU and GFP. (C) Percentage of proliferating epicardial $\mathrm{GFP}^{+}$cells. $\mathrm{n}_{\mathrm{WT}}=5, \mathrm{n}_{\mathrm{X} 136}=6$. Scale bar $100 \mu \mathrm{m}$. (G-F) 3D volume rendering of the apical injured site of $7 \mathrm{dpci} \operatorname{Tg}(f l i l a: G F P) / \operatorname{Tg}(m y l 7: m R F P)$ hearts. Yellow lines indicate the injured area and heart cartoon the $\mathrm{x} / \mathrm{y} / \mathrm{z}$ axes. (F) Quantification of the $\mathrm{GFP}^{+}$cells volume inside the $\mathrm{RFP}^{-}$area. t-test, $\mathrm{n}_{\mathrm{WT}}=3, \mathrm{n}_{\mathrm{X} 136}=4$. Scale bar $300 \mu \mathrm{m}$. 


\section{Cardiomyocytes proliferation is transiently affected in injured X136 hearts}

We further examined the proliferations of $\mathrm{CM}$, since it has been reported that this feature was affected upon ventricular resection in a Cav1-KO zebrafish model (Cao et al., 2016). Therefore, we analysed the $\mathrm{CM}$ adjacent to the injured area at $7 \mathrm{dpci}$, because they are highly proliferative (Bednarek et al., 2015; Wang et al., 2011). Quantification of BrdU incorporation in CM revealed that they proliferated less in X136 hearts (Figure 37A-C). To confirm this result, we examined CM proliferation in the X166 animals and we made the same observation (Sup. Figure 13). We then wondered why that difference in CM proliferation did not affect the regeneration process and thus, we examined the proliferation status of CM 14dpci (Figure 37D-F). The analysis showed that CM in the X136 hearts proliferated with the same rate as in WT at 14dpci. Taken together, these data indicate that loss of caveolae leads to a transient effect in CM proliferation at $7 \mathrm{dpci}$, that was normalized by $14 \mathrm{dpci}$, leading to normal cardiac regeneration at $90 \mathrm{dpci}$.
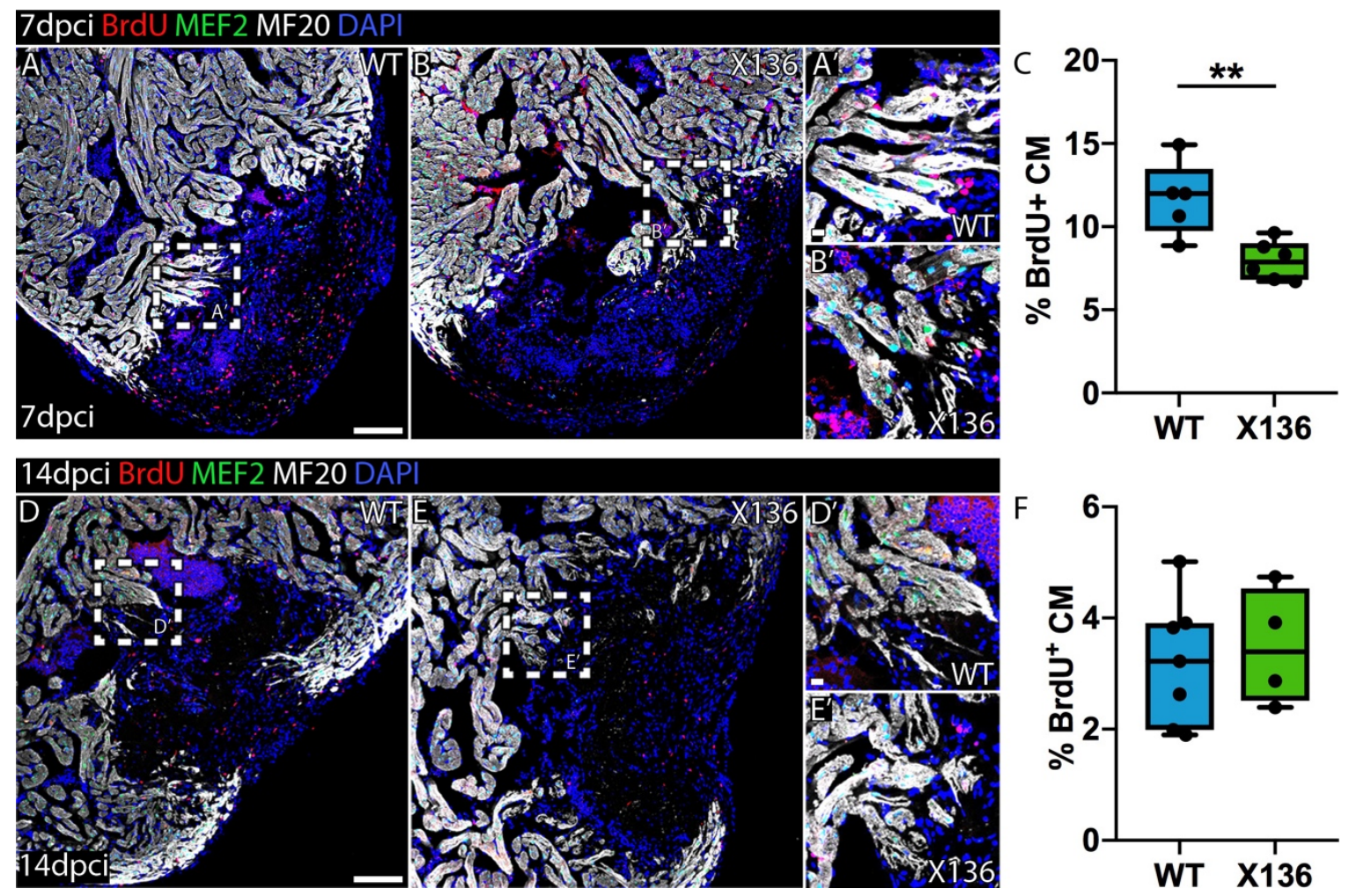

Figure 37. CM proliferation upon injury in X136 hearts

(A-B) 7dpci WT and X136 hearts were labelled for BrdU, MEF2 and MF20. A' and B' magnifications of the dashed areas in A and B. (C) CM proliferation rate was addressed by quantifying the $\mathrm{BrdU}^{+} / \mathrm{MEF}^{+}$nuclei to the total $\mathrm{CM}$ number in a $100 \mu \mathrm{m}$ radius around the injured area. $\mathrm{n}_{\mathrm{WT}}=5, \mathrm{n}_{\mathrm{X} 136}=6$, t-test, $* * \mathrm{P}<0.01$. (D-E) Immunostaining of $14 \mathrm{dpci} \mathrm{WT}$ and $\mathrm{X} 136$ hearts for BrdU, MEF2 and MF20. (F) Quantification of CM proliferation 14dpci. $\mathrm{n}_{\mathrm{WT}}=7, \mathrm{n}_{\mathrm{X} 136}=4$. Scale

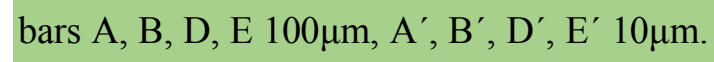




\section{Impaired heart function in the X136 animals}

Cavl deficient mouse have been reported to show decreased systolic function (Cohen et al., 2003; Zhao et al., 2002). To address this issue in our cav1-KO zebrafish model, we examined heart function using ultrasound. We analysed adult WT and X136 animals by echocardiography (Figure 38). Our analysis revealed that cavl-KO hearts were less efficient in pumping blood than the WT hearts, since their ejection fraction (EF) was significantly decreased (Figure 38A). Additionally, the heart rate of the X136 animals was significantly lower than in WT littermates (Figure 38B). Thus, the X136 hearts had decreased EF and heart rate, indicating that caveolae are important for normal cardiac function.

\section{$\square$ WT $\square$ X136}
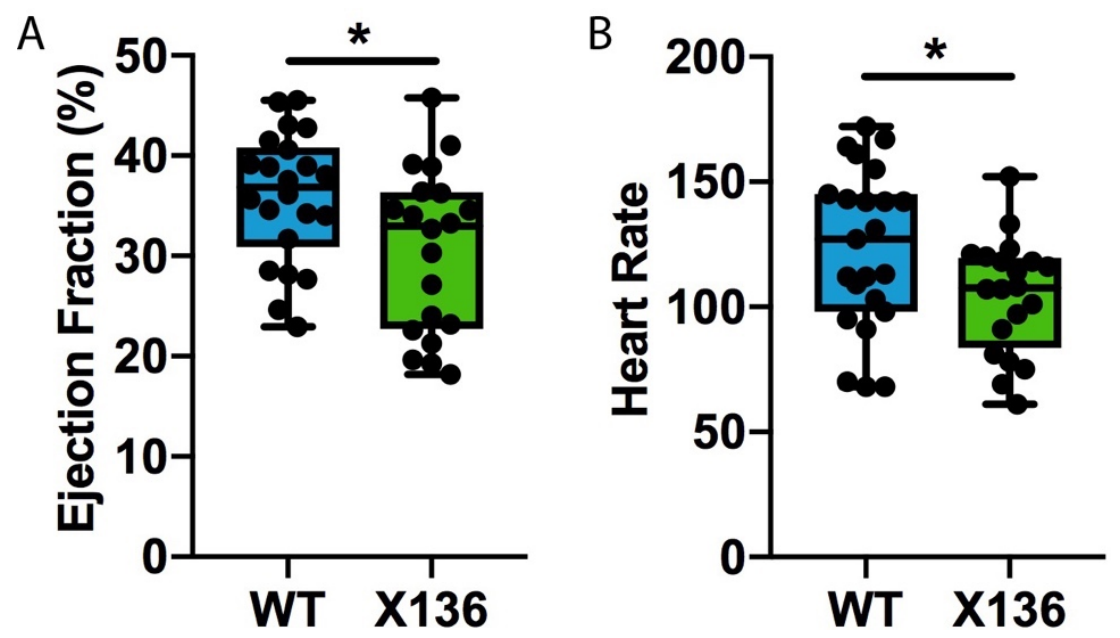

Figure 38. Echocardiography analysis of WT and X136 hearts

(A) Quantification of the EF in WT and X136 hearts. $\mathrm{n}_{\mathrm{WT}}=22, \mathrm{n}_{\mathrm{X} 136}=20$. (B) Comparison of the heart rate in $\mathrm{WT}$ and $\mathrm{X} 136$ animals. $\mathrm{n}_{\mathrm{WT}}=23, \mathrm{n}_{\mathrm{X} 136}=20$. t-test, $* \mathrm{P}<0.05$.

\section{Depletion of caveolae affects cardiac elasticity}

Caveolae have been showed to provide protection against mechanical stress (Gervásio et al., 2011; Sinha et al., 2011). Since the biomechanical properties of cells influence the behaviour of tissues (Mathur et al., 2001) and caveolae are involved in mechanoprotection, we investigated the mechanical response of WT and cavl-KO cardiac tissue, trying to get an insight into the differences observed in cardiac function by echocardiography. We have used atomic force microscopy (AFM) to determine the mechanical properties of the adult WT and cavl-KO cardiac tissue. Freshly isolated hearts were placed on an agarose gel and the apex of the ventricle was oriented to the direction of the cantilever and procced to take the measurements (Figure 39A-D).

We found that X136 animals had significantly increased cardiac tissue stiffness when compared to WT (Figure 39E). Applying the same force, the WT hearts were deformed 1.5 times 
more that the X136, without causing permanent deformation, since the curves returned to zero (Figure 39F). Arguably, the difference in elasticity and the increase in stiffness can only be attributed to the depletion of the membrane reservoirs, caveolae. Importantly, the sensitivity of the method reveals that changes in epicardial and cortical myocytes are responsible for the switch in stiffness we detected. Hence, absent of caveolae, due to the loss of the broad and robust expression of Cav1 in the ventricle, results in increased tissue stiffness.
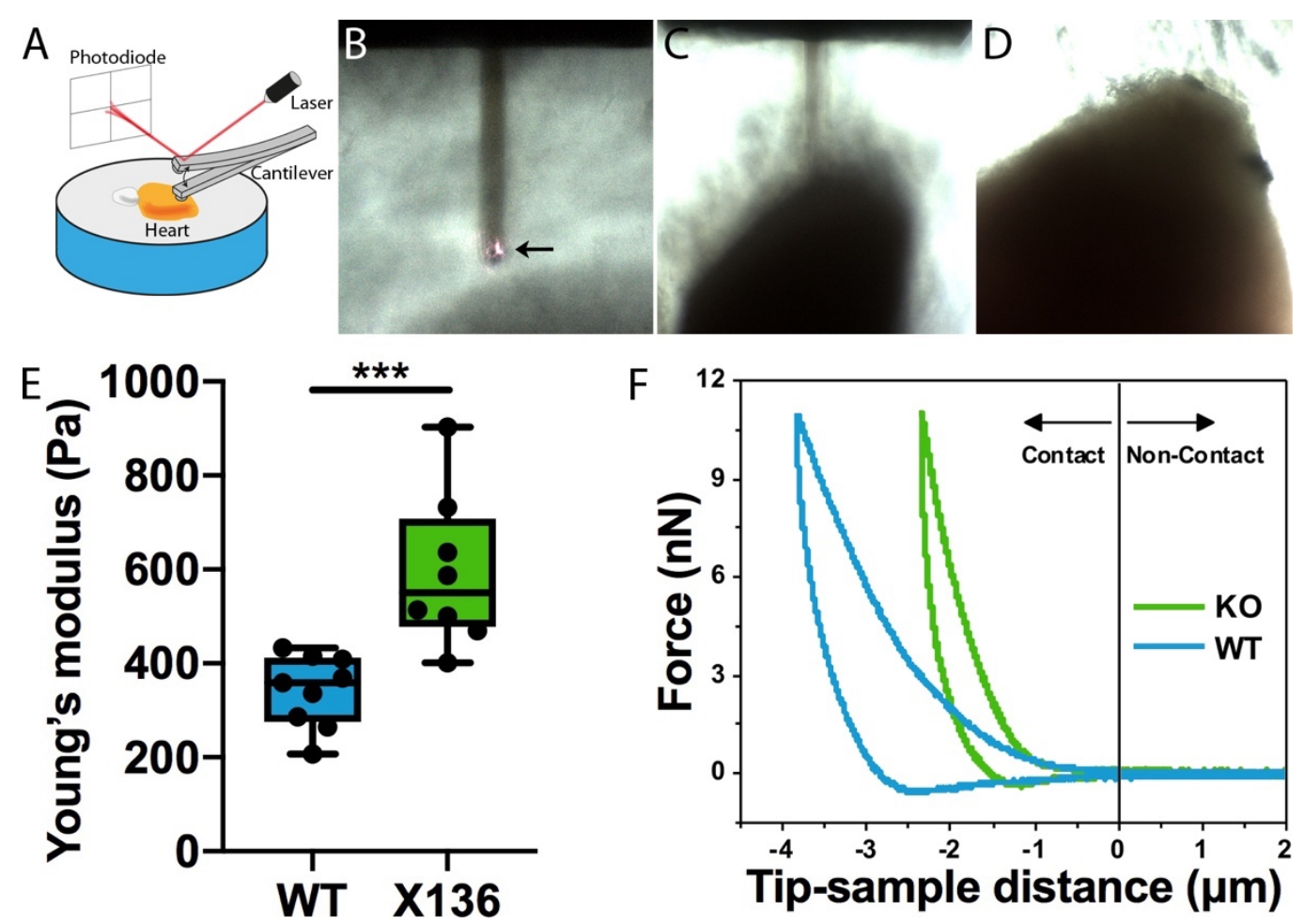

Figure 39. Loss of caveolae leads to stiffer cardiac tissue

(A) Illustration of AFM set-up. (B) Arrow indicates the cantilever and the laser beam. (C-D) the apex of the ventricle was orientated in respect to the cantilever and used for taking measurements. (E) Biomechanical characterization of WT and X136 ventricles as measured by AFM and expressed in Young's moduli. $\mathrm{n}_{\mathrm{WT}}=9, \mathrm{n}_{\mathrm{X} 136}=8, \mathrm{t}$-test, $* * * \mathrm{P}<0.001$. (F) Force - distance graph for the indentation and retraction of the cantilever over the ventricular surface. 
Supplementary Figures 


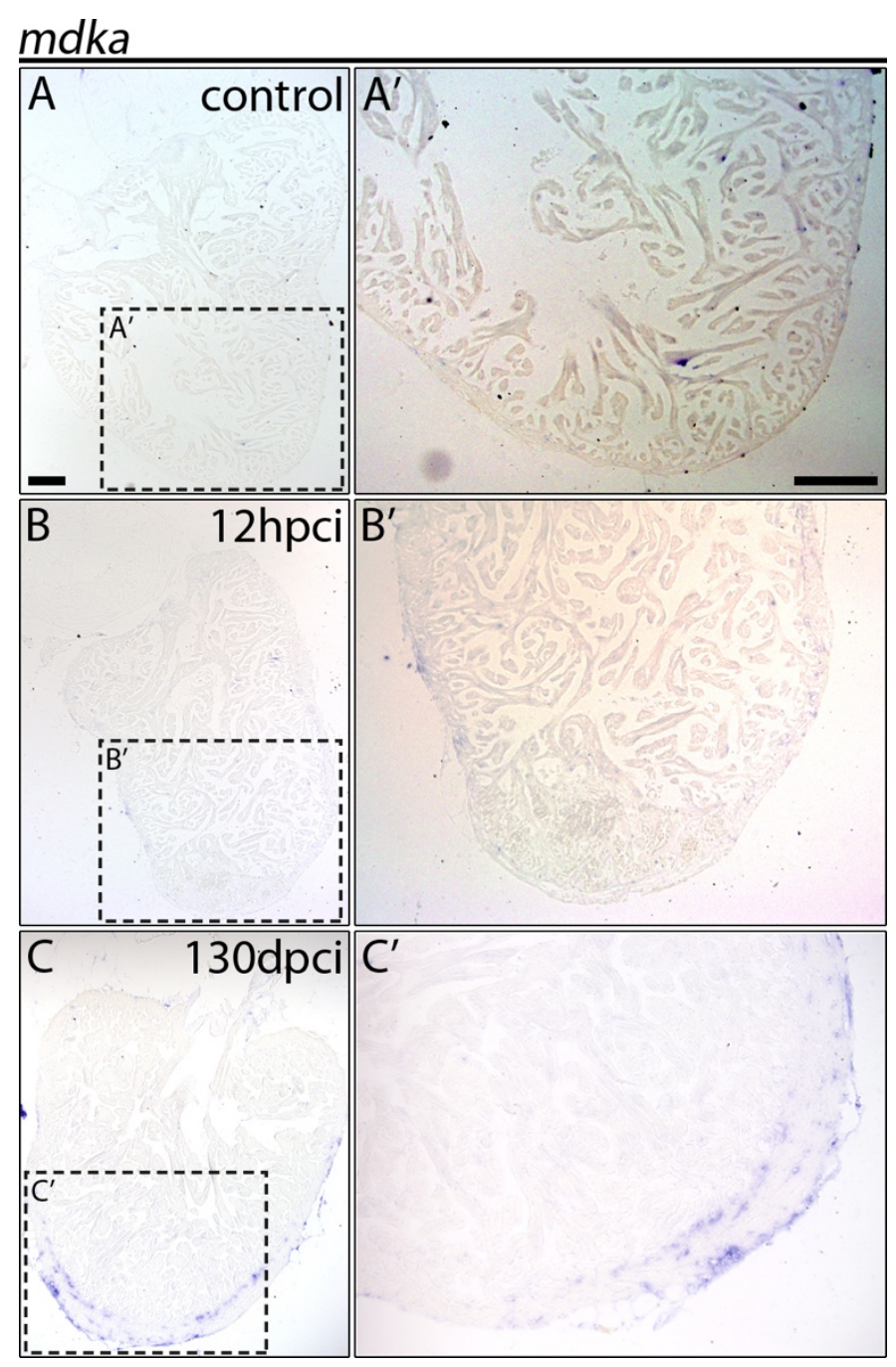

Supplementary Figure 1. Expression of $m d k a$ in control and regenerating hearts

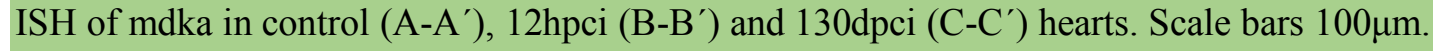




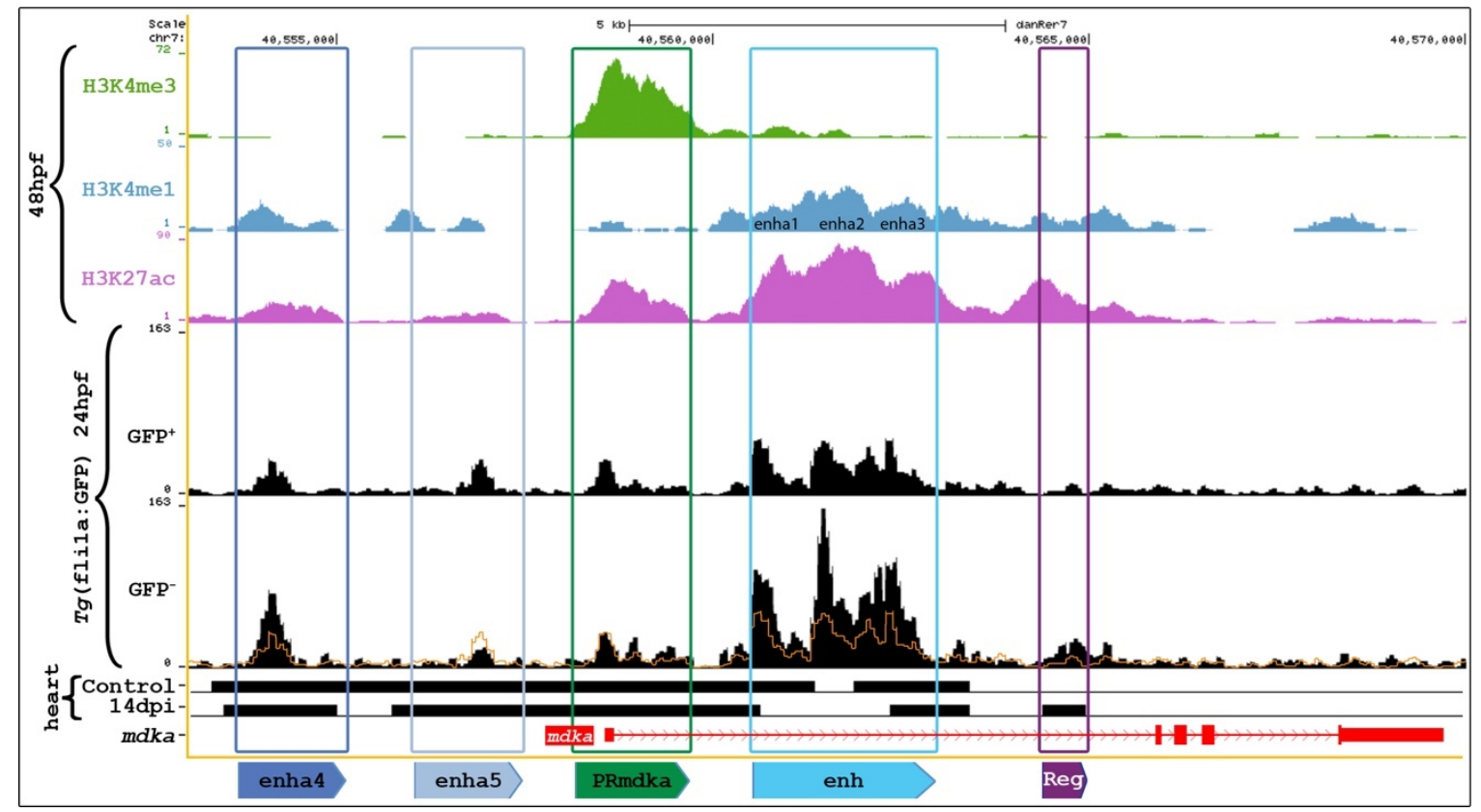

\section{Supplementary Figure 2. Epigenetic marks in mdka locus $48 \mathrm{hpf}$}

Snapshot of the UCSC genome browser with tracks showing methylation (H3K4me3/1) and acetylation (H3K27ac) marks from 48hpf embryos (peaks) and from control or 14dpi hearts (black boxes) and peaks of ATAC-seq illuminating the accessible genomic regions from $\mathrm{GFP}^{+}$or $\mathrm{GFP}^{-}$ cells from $24 \mathrm{hpf} \operatorname{Tg}\left(f l i l a\right.$ :GFP) embryos. $m d k a$ locus in red. The orange line in $\mathrm{GFP}^{-}$track is the outline of the $\mathrm{GFP}^{+}$peaks to illustrate the difference in intensity. All cloned regions in coloured boxes and the corresponding genomic regions in the associated coloured outlines.

\section{Tg(enh-mdka:GFP)}

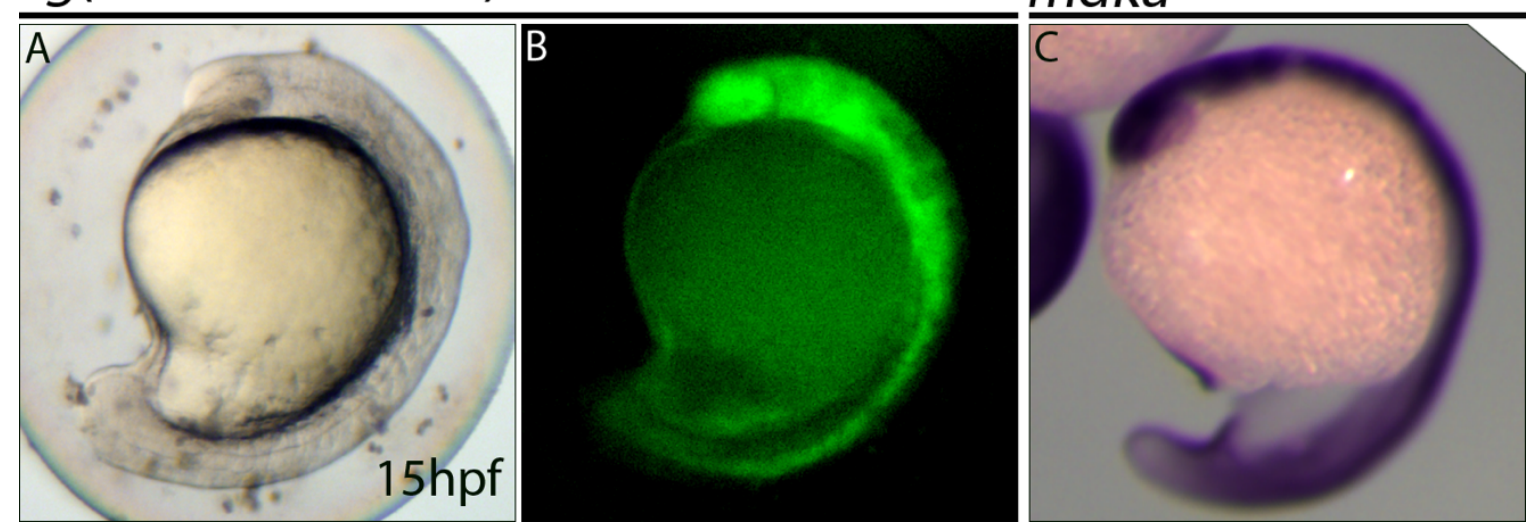

Supplementary Figure 3. Tg(enha-mdka:GFP) expression at 15hpf

Brightfield (A) and fluorescent (B) images of $15 \mathrm{hpf} T g(e n h-m d k a: G F P)$ embryo. (C) $m d k a$ WMISH in 15 hpf embryo. 


\section{Tg(enh-mdka:GFP)}

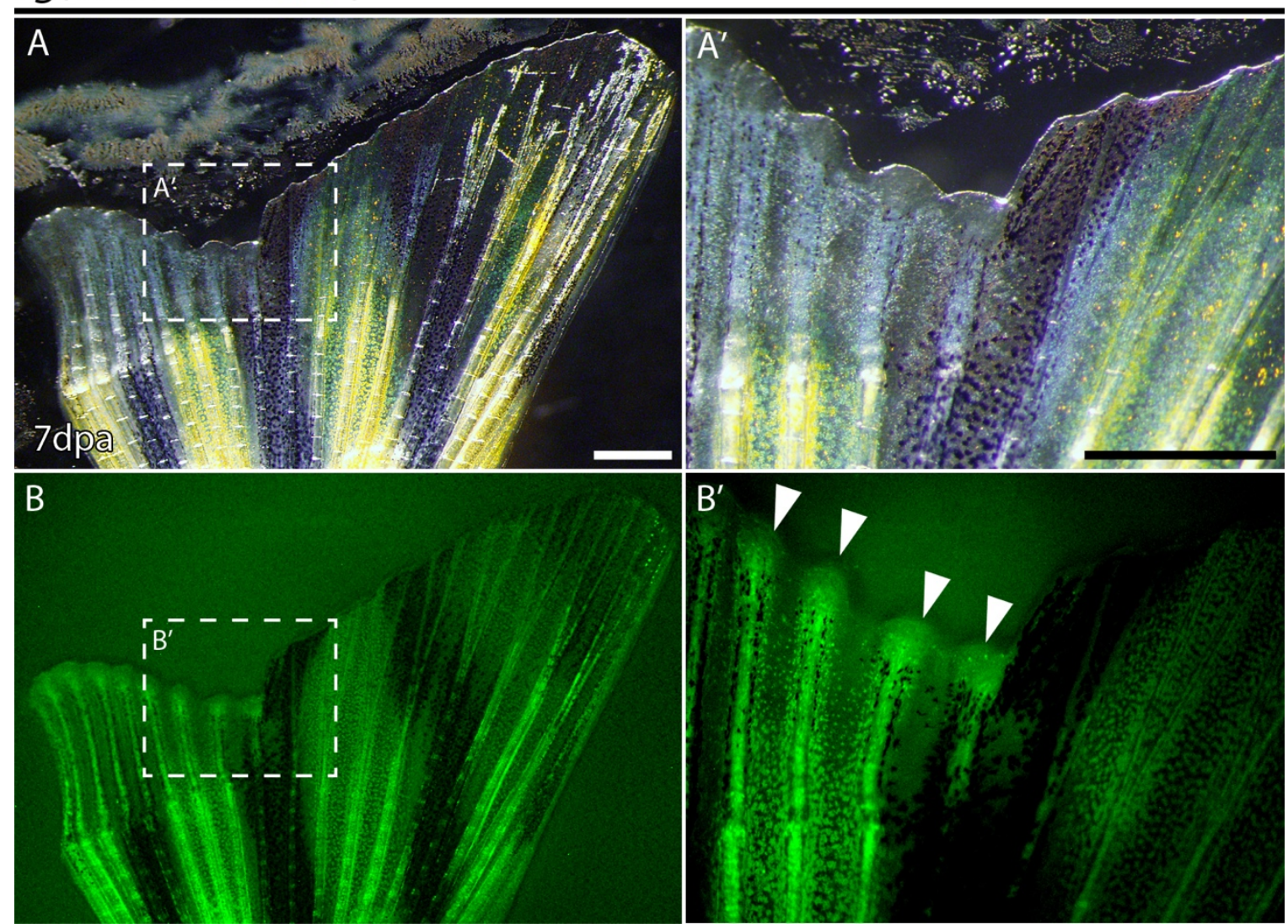

Supplementary Figure 4. Tg(enh-mdka:GFP) animals show GFP expression only in regenerating fin

(A, B) The dorsal halve of the fin was amputated and the ventral was kept intact for control of the GFP expression. $\left(\mathrm{A}^{\prime}, \mathrm{B}^{\prime}\right)$ Magnifications of the marked areas in A and B. Arrowheads indicate GFP in the regenerating fins. 

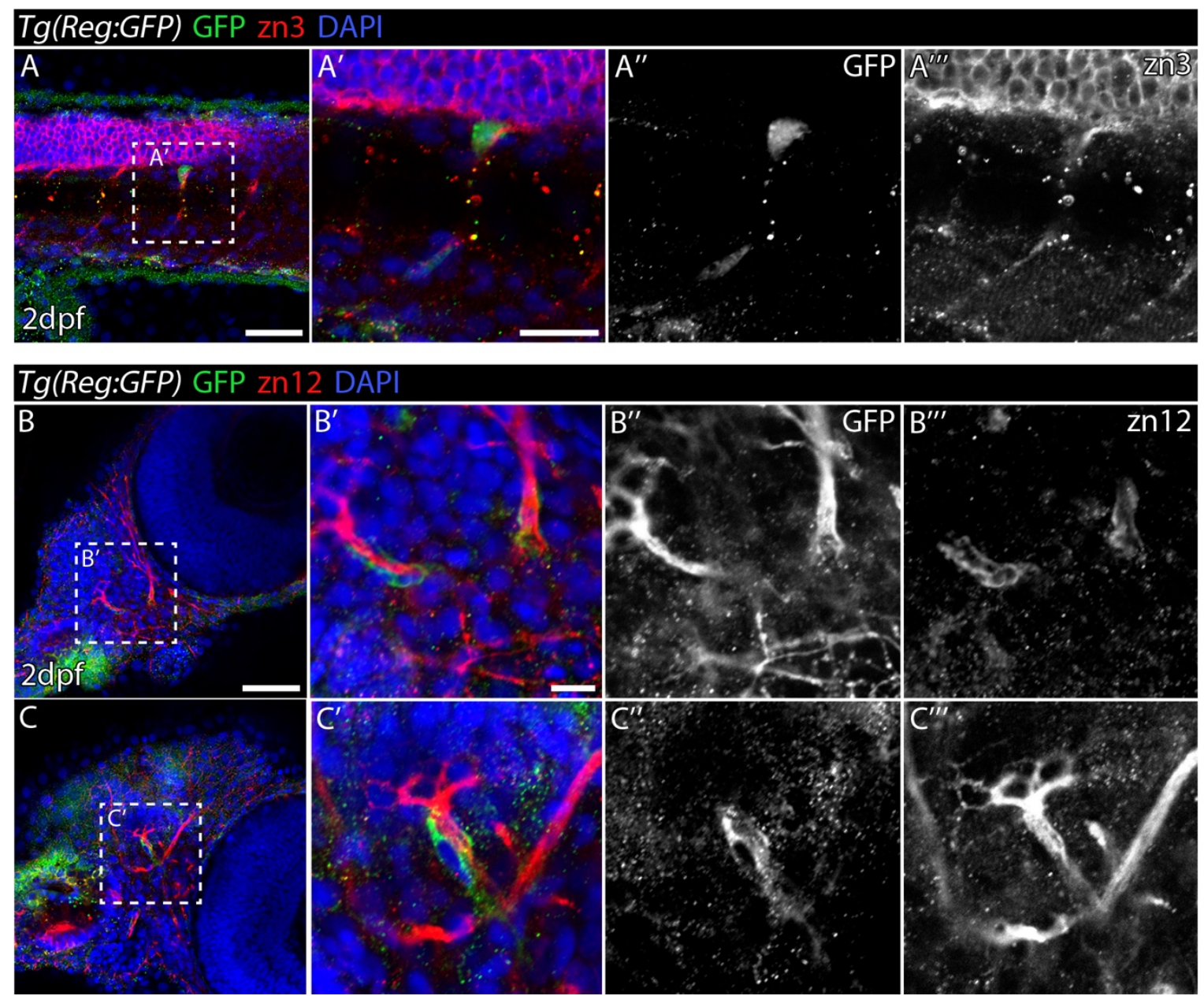

Supplementary Figure 5. Immunofluorescence of GFP and zn3 or zn12 in $\operatorname{Tg}(\operatorname{Reg}: G F P)$ embryos

Whole-mount immunostaining of GFP and zn3 (A-A"') or zn12 (B-C"') of 2dpf embryos. (A'$\left.A^{\prime \prime \prime}\right)$ Higher magnification of the boxed areas in A. (B-C"') Images showing cranial neuromasts in red and GFP expression in supporting cells. $\left(\mathrm{B}^{\prime}-\mathrm{B}^{\prime \prime \prime}\right)$ and $\left(\mathrm{C}^{\prime}-\mathrm{C}^{\prime \prime \prime}\right)$ Magnifications of the

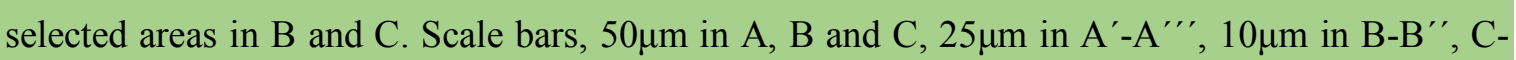
$\mathrm{C}^{\prime \prime}$. 

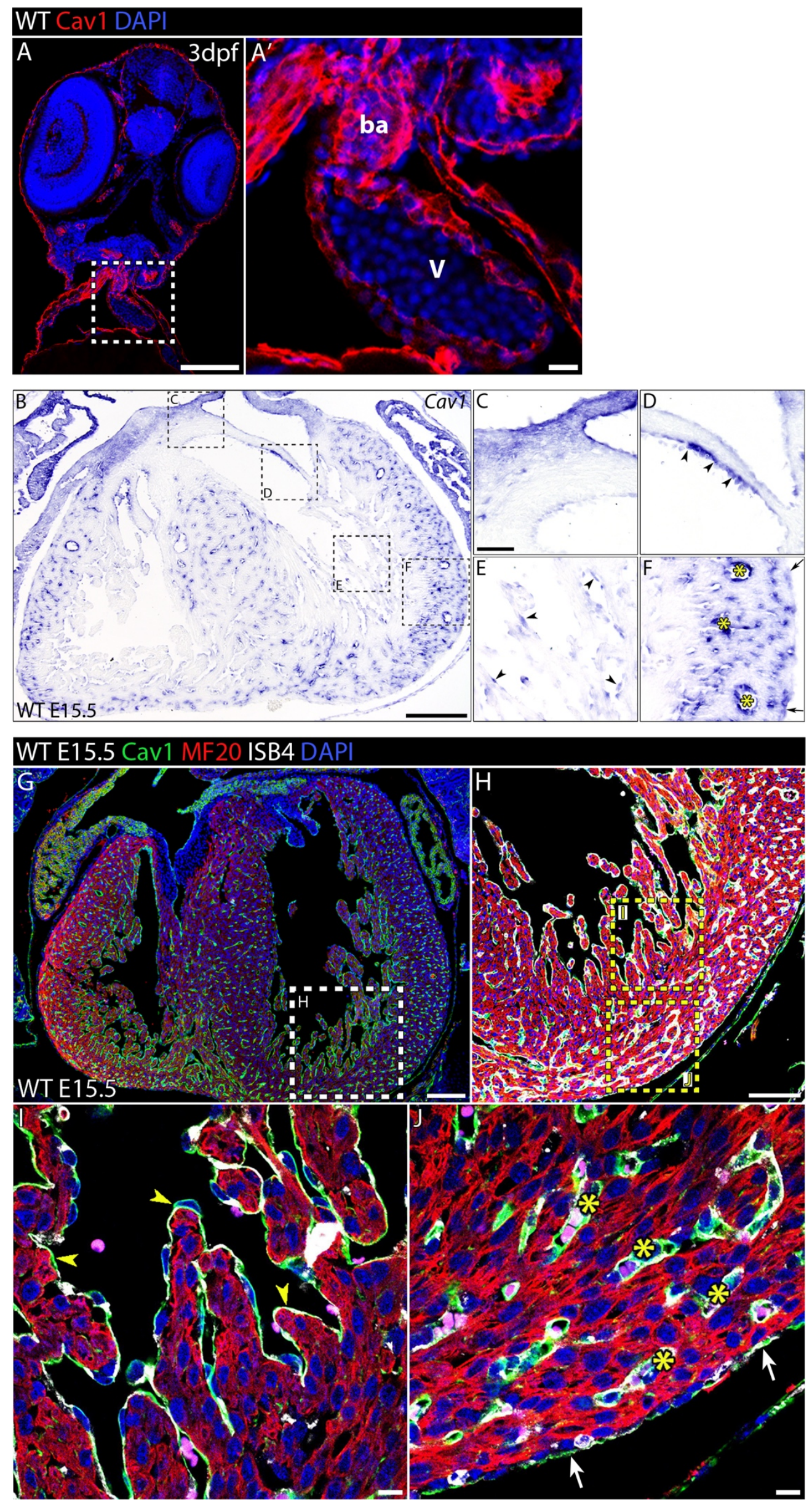


\section{Supplementary Figure 6. Cav1 expression in embryonic zebrafish and mouse heart}

(A-A') Immunostaining of Cav1 in 3dpf zebrafish embryo. (A') Higher magnification of the dashed area in A. ba $=$ bulbous arteriosus, $\mathrm{v}=$ ventricle. Scale bars, A $100 \mu \mathrm{m}, \mathrm{A}^{\prime} 50 \mu \mathrm{m}$. (B-F) In situ hybridization of Cavl in mouse embryonic E15.5 heart. (C-F) Higher magnifications of the corresponding areas in B. (D and E) Arrowheads indicate Cavl in endocardium. (F) Arrows and asterisks show Cavl in epicardium and vasculature, respectively. Scale bars, B $100 \mu \mathrm{m}, \mathrm{C}-\mathrm{F} 50 \mu \mathrm{m}$. (G-J) Mouse embryonic heart labelled for CAV1, isolectinB4 (ISB4) and MF20 (CM). (H) Higher magnification of the dashed area in G. (I-J) Magnifications of dashed areas in H. Arrowheads, asterisks and arrows indicate CAV1 in the endocardium, endothelium and epicardium, respectively. Scale bars, G and H $100 \mu \mathrm{m}$, I and J $50 \mu \mathrm{m}$.

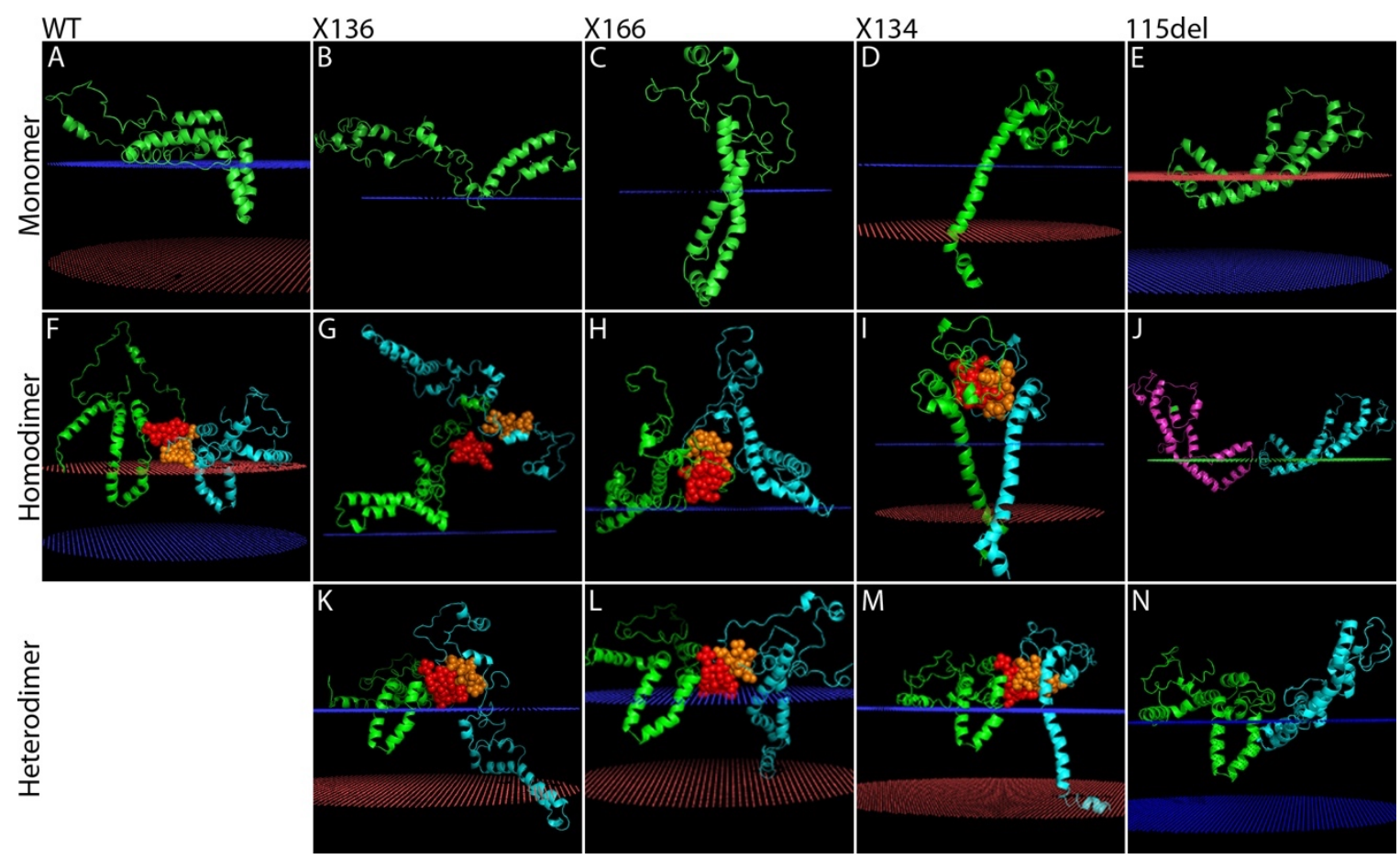

Supplementary Figure 7. In silico 3D modelling and analysis of the Cav1 mutant proteins

(A-E) Intramembrane Analysis. 3D models of the Cav1 WT and mutant proteins showing the putative a-hairpin membrane insertion and the embedded residues with the membrane layers presented as red and blue dots planes. (A) The modelling predicted a deep (half membrane) ahairpin insertion for Cav1. (B) This model failed to predict a membrane insertion, only minimal contact with embedded residues around the scaffolding domain and not in the predicted putative membrane domain. (C) This model predicted an insertion of the initial C-terminus scaffolding domain and polymerization domain and a spatial reorientation of the protein to make the transmembrane (TM) domain ordinary to the membrane layer insertion. This, probably, is an unrealistic model. (D) The model predicted a putative trans-membrane insertion. (E) The analysis predicted an insertion or deep embedded membrane domain. This insertion is minor deeper than in 
the WT. (F-J) Analysis of homodimer. Views of the 3D models of homodimers showing the A chain in green, the B chain in cyan (or magenta), the putative a-hairpin membrane insertion and the dimer interface as red or orange spheres. (F) The predicted homodimer fulfils the topological membrane restrictions and the spatial interface coordinates. The slightly out-of-membrane position of the A chain probably is an artefactual effect because the algorithm considers the membrane as a rigid body, however, this distortion maybe compatible with the proposed membrane curvature/deformities for caveolae formation. (G) The prediction was that is not possible the homodimer formation by the theoretical interface. The analysis indicates that there is not dimer but only a transient dimer form for this mutant protein that is matching to a peripheral membrane protein. (H) This model is compatible with a superficial or slightly embedded protein. (I) The predicted homodimer dimerizes by the theoretical interface and also predicted to be a TM protein. (J) The predicted homodimer dimerizes by the theoretical interface and also predicted to be an imbedded protein. (K-N) Analysis of heterodimer. Cartoon representations the Cav1 and mutant's 3D heterodimer models, showing the WT in green and mutants in cyan, the membrane layer as red and blue dots and the dimers interface as red/orange spheres. (K) The predicted heterodimer fulfilled the spatial interface coordinates and predicted a superficial membrane dimer or the mutant chain to be slightly embedded. Additionally, the server failed to predict a correct model of inserting the mutant very deep in membrane as TM. This representation is an unrealistic model. (L) The analysis resulted in a superficial membrane dimer or slight embedding for the mutant chain. The representation of the very deep membrane insertion for the mutant is an unrealistic model. (M) The predicted heterodimer is inserted or TM dimer. $(\mathrm{N})$ The analysis predicted an PM embedded or TM membrane dimer and all the dimers are possible and stable. Overall, all dimers, excluded the X136 homodimer are possible and stable considering the energy requirements and the stability of each one. The 3D modelling predicted that the energy of the X136 homodimer is very high, resulting in great instability and suggested that this mutant does not dimerize making it the best candidate among the other mutations. 

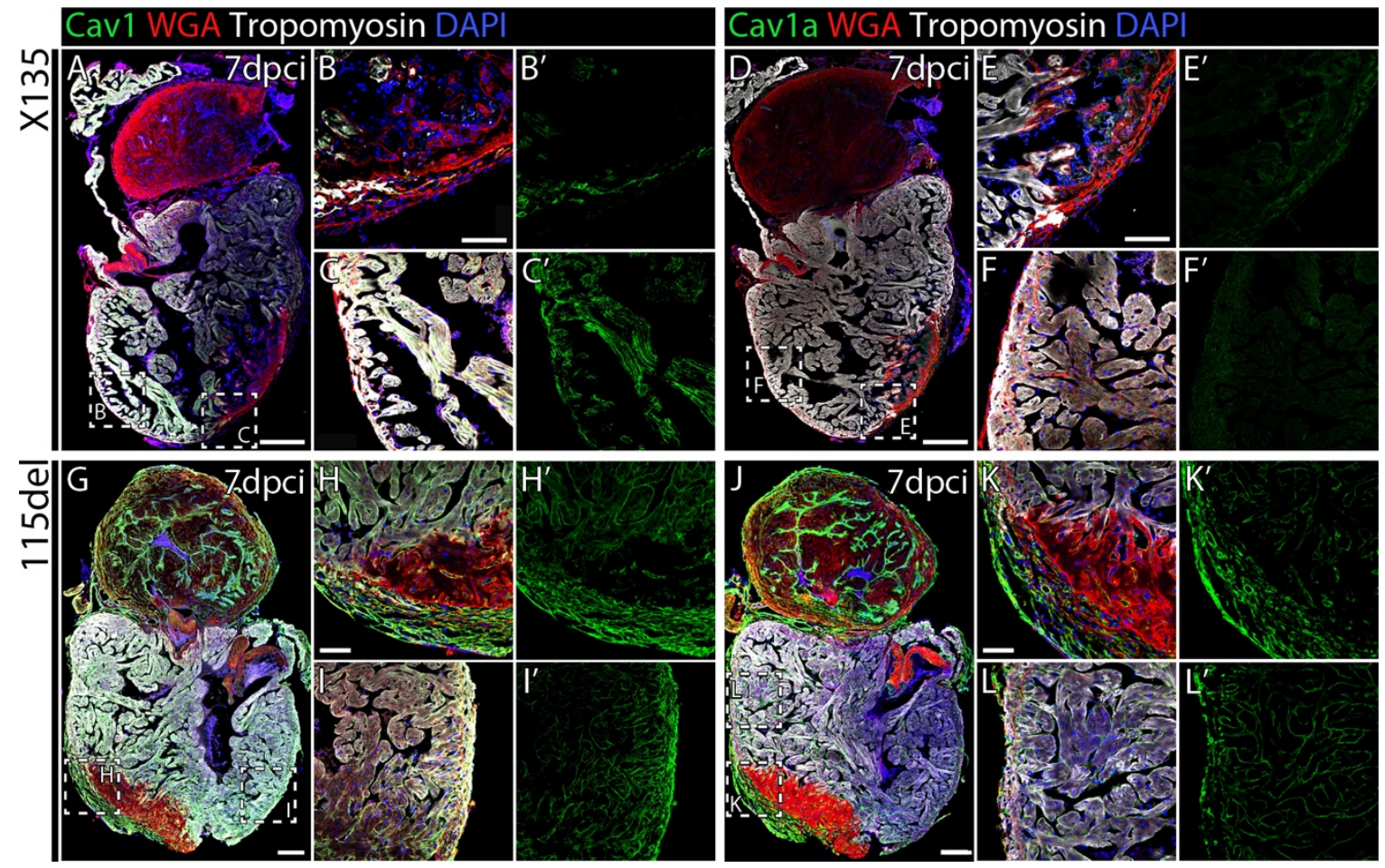

Supplementary Figure 8. Expression analysis of Cav1 in X135 and 115del hearts

(A-L') Immunostaining for Cav1 or Cav1a in X135 (A-F) and 115del (G-L) 7dpci $T g$ (wt $1 b$ :GFP) hearts. B-C'; E-F'; H-I' and K-L' magnifications of the selected areas in A, D, G and J, respectively. Scale bars $100 \mu \mathrm{m}$ in $\mathrm{A}, \mathrm{D}, \mathrm{G}$ and $\mathrm{J} ; 50 \mu \mathrm{m}$ in the rest. 
A

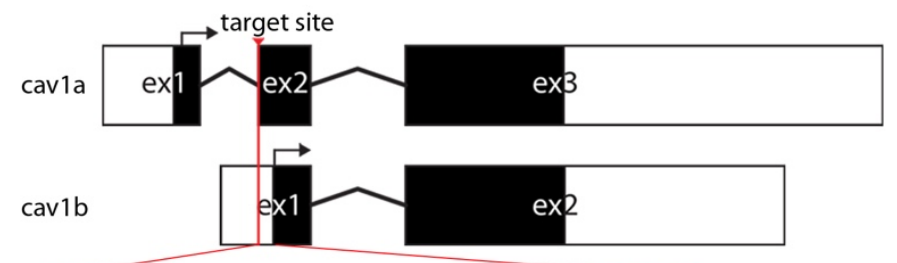

B WT TTTGGAACCGCAGGAATACGCTCACTCGCCGTTTATCAGGAAACAGGGGACATTTACAAACCAAACAATAAAGAAATGG

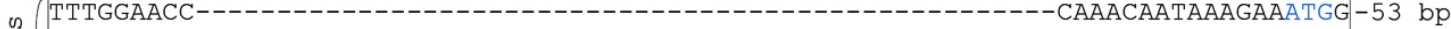

:

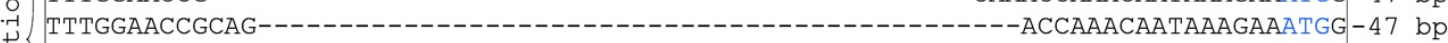

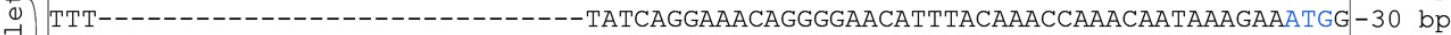

के TTTGGAA---------------------------------ACAGGGGAACATTTACAAACCAAACAATAAAGAAATGG -35 bp TTTGAAAC----_-

$\star * \star *$

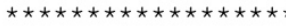

WT TTTGGAACCGCAG-----------------GAATACGCTCACTCGCCGTTTATCA

ins $\{$ TTTGGAACCGCAGTCA----------ATACGAATACGCTCACTCGCCGTTTATCA +7 bp TTTGGAACCGCAGGAATACGCTCTTTCTTTGGAATACGCTCACTCGCCGTTTATCA +18 bp

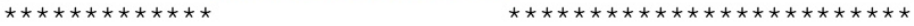

C Cav1a PEEEYAHSPFIRKOGNIYKPNNKEMDNDS INEKTLODVHTKE IDLVNRDP F19fSX21 PEEEYAHSPKQ.

F19fSX53 PEEEYAHSP-NOTIKKWITTASTKRHFRMSTPRRLTWSTETOSI.

E13fsX16 PEETKQ.

E13 P18del PEE------FIRKQGNIYKPNNKEMDNDS INEKTLQDVHTKEIDLVNRDP

Y14 fsX23 EEETGEHLOTKO.

E13fSX54 PEEGNRGTFTNQTIKKWITTASTKRHFRMSTPRRLTWSTETQSI.

Cavla PEEE------YAHSPFIRKQGNIYKPNNKEMDNDS INEKTLQDVHTKEID

E13fsX34 PEESIRIRSLAVYQETGEHLQTKQ.

E13_Y14ins PEEEYALSLEYAHSPFIRKQGNIYKPNNKEMDNDS INEKTLQDVHTKEID

Cavla intron1
cavla exon2
cav1b 5'region
cav1b exon1
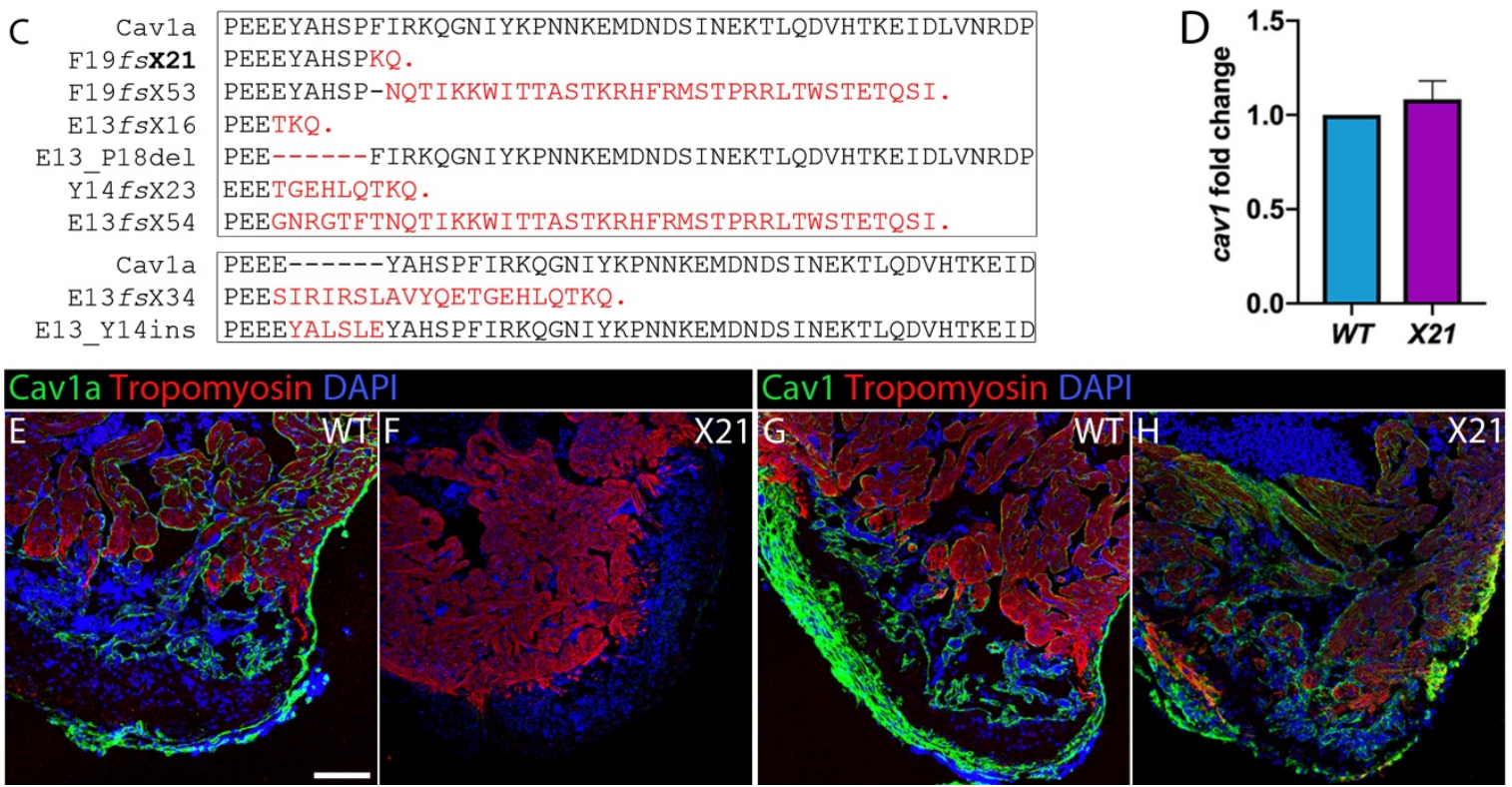

Supplementary Figure 9. cav1a deletion by CRISPR/Cas9 and Cav1 expression analysis in cavla-KO hearts

(A) Schematic representations of cavl locus with the two transcripts, cavla and cavlb. Target site of the guide RNA in red arrowhead. ex = exon. (B) Identified genetic mutations. Hyphens indicate deletions and red letters insertions. Blue letters $=c a v 1 b$ start codon. $\mathrm{bp}=$ base pairs. $(\mathrm{C})$ Predicted effect on protein. Red hyphens indicate deletions and red letters the novel amino acids. (D) cav1 qPCR analysis in 2 dpf embryos. Mean \pm S.D., t-test. (E-H) Immunostaining of Cav1a or Cav1 in heart sections from WT and X21. Scale bar $100 \mu \mathrm{m}$. (I-J) Fluorescent labelling of WT and X21 hearts $7 \mathrm{dpci}$ with BrdU and aldh1a2. Scale bar $100 \mu \mathrm{m}$. 

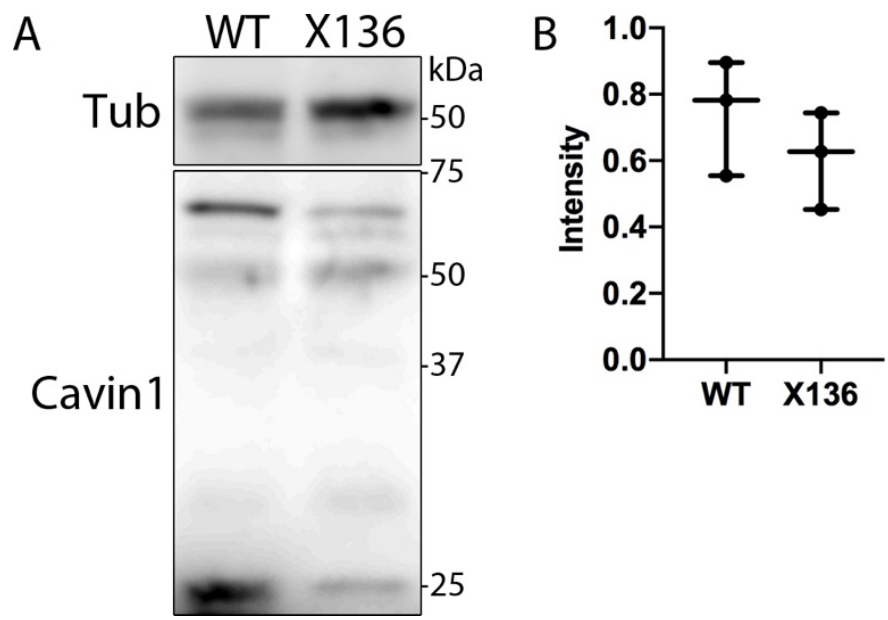

\section{Supplementary Figure 10. WB analysis of Cavin1 in X136 hearts}

(A) WT and X136 hearts were analysed by WB using an antibody against Cavin1. Tubulin (Tub) served as loading control. $\mathrm{kDa}=$ kilodalton. (B) Quantification of Cavin1 WB intensity normalized to Tub. $\mathrm{n}_{\mathrm{WT}}=\mathrm{n}_{\mathrm{X} 136}=3$. t-test.
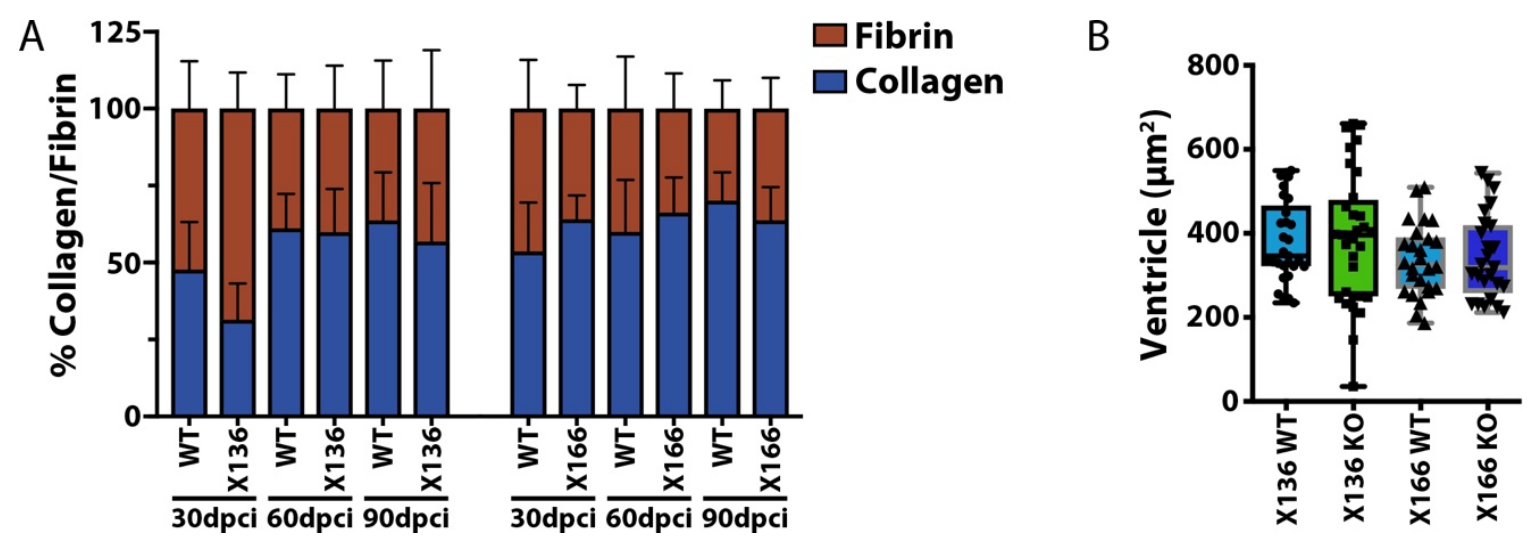

Supplementary Figure 11. Analysis of collagen and fibrin of the injured area and ventricular volume

(A) Percentages of the collagen and fibrin of the injury 30-, 60- and 90dpci in WT, X136 and X166 cryoinjured hearts. Two-way ANOVA. $\mathrm{n}_{\mathrm{WT}(\mathrm{X} 136)} 30-, 60-, 90 \mathrm{dpci}=10,9,9 ; \mathrm{n}_{\mathrm{X} 136} 30-, 60-, 90 \mathrm{dpci}$ $=10,10,12 ; \mathrm{n}_{\mathrm{WT}(\mathrm{X} 166)} 30-, 60-, 90 \mathrm{dpci}=9,9,8 ; \mathrm{n}_{\mathrm{X} 166} 30-, 60-, 90 \mathrm{dpci}=7,8,11$. (B) Ventricular size of all hearts analysed by AFOG staining. t-test. $\mathrm{n}_{\mathrm{WT}(\mathrm{X} 136)}=29 ; \mathrm{n}_{\mathrm{X} 136}=32 ; \mathrm{n}_{\mathrm{WT}(\mathrm{X} 166)}=26 ; \mathrm{n}_{\mathrm{X} 166}$ $=25$. 

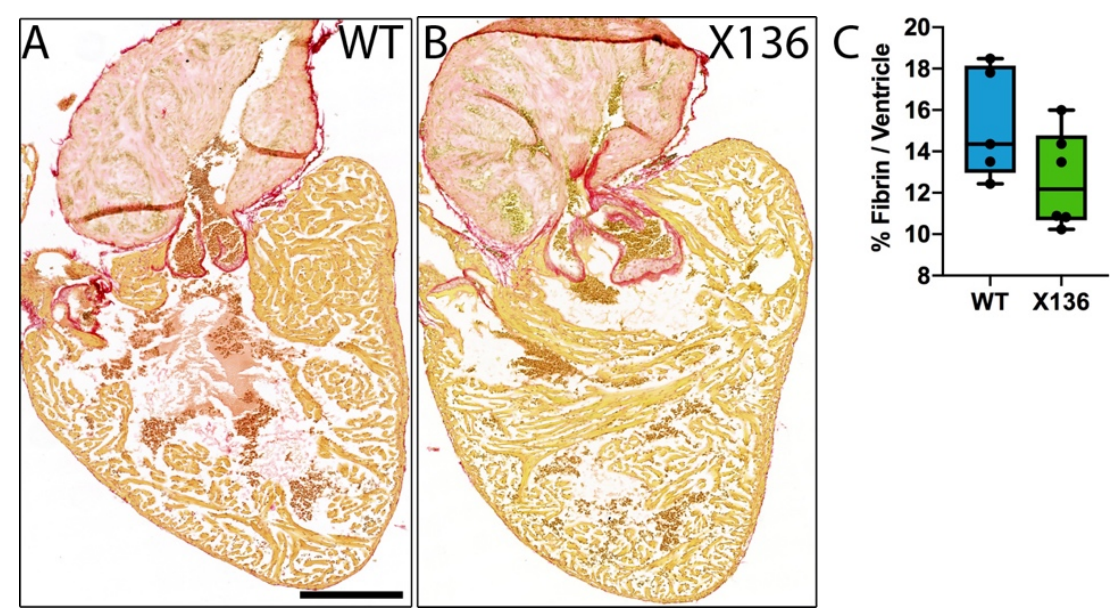

Supplementary Figure 12. Analysis of interstitial fibrosis in WT and X136 hearts

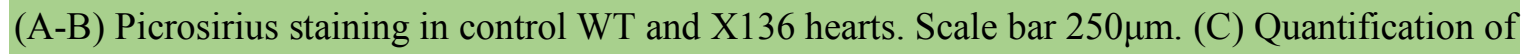
the red-labelled fibers in the ventricle. $\mathrm{t}$-test, $\mathrm{n}_{\mathrm{WT}}=5, \mathrm{n}_{\mathrm{X} 136}=6$.

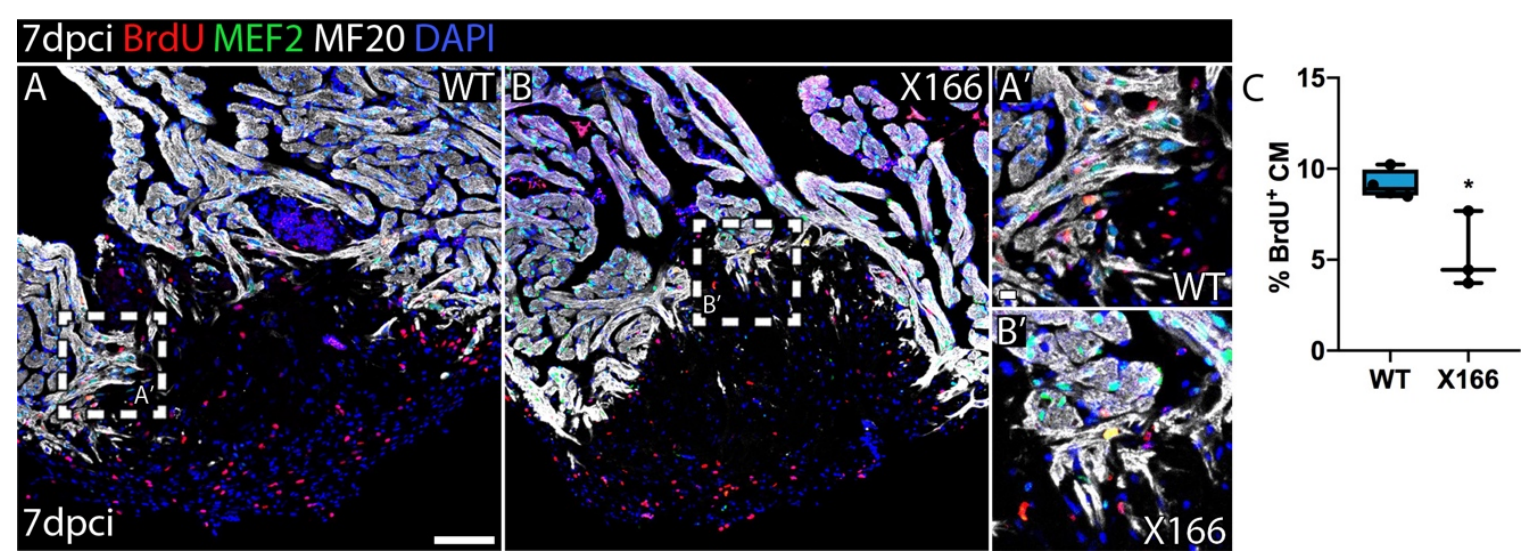

Supplementary Figure 13. X166 CM proliferation upon injury

(A-B') 7dpci WT and X166 hearts were labelled for BrdU, MEF2 and MF20. (A'-B') Higher

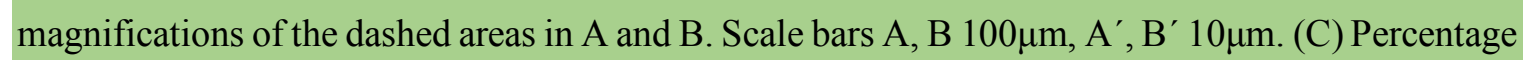
of the $\mathrm{BrdU}^{+} \mathrm{CM}$ to the total number of the $\mathrm{CM}$ in a $100 \mu \mathrm{m}$ area surrounding the damaged tissue. $\mathrm{n}_{\mathrm{WT}}=4, \mathrm{n}_{\mathrm{X} 136}=3$. t-test, $* \mathrm{P}<0.05$. 
Discussion 
Midkine is a highly conserved secreted neurite-promoting factor that get activated upon cardiac damage (Horiba et al., 2006; Hugosson et al., 2014; Kadomatsu et al., 1990; Winkler et al., 2003). In heart injury and disease, Mdk in involved in ECM regulation, neutrophils recruitment and revascularization (Fukui et al., 2008; Horiba et al., 2006; Weckbach et al., 2019). In a previous study in the laboratory, we found midkine- $a$ upregulated in a microarray analysis of regenerating hearts (González Rajal, 2012). Here, we generated the $m d k a-K O$ zebrafish strain to study the role of Mdka in heart regeneration.

\section{Mdka expression after injury}

Expression analysis of $m d k a$ revealed that $m d k a$ is not expressed in intact adult heart but it is highly upregulated upon injury. We found that the onset of $m d k a$ expression was $1 \mathrm{dpci}$ in the entire epicardial layer, similarly to $3 \mathrm{dpci}$, and that by $7 \mathrm{dpci}$, the expression was restricted to the epicardial cells surrounding the damaged tissue. After the end of heart regeneration, $m d k a$ was also detected in the newly formed compact layer possibly in vasculature, as well as in the zone between the cortical and trabecular myocardium. This distinct spatio-temporal pattern of expression is in contrast with data from injured rodent hearts, which show $M d k$ expression in CM and/or endothelial cells, but not in epicardial cells (Fukui et al., 2008; Obama et al., 1998). In adult mice, the epicardium becomes activated after MI and re-expresses embryonic genes such as RA and WT1 (van Wijk et al., 2012), similarly to zebrafish (González-Rosa et al., 2012; Kikuchi et al., 2011b). However, transcriptional analysis of mouse injured hearts, reveals that Mdk is upregulated in leukocytes. Additionally, that is enriched in CM, fibroblasts and endothelial cells of adult hearts after MI compared to post-natal MI (Quaife-Ryan et al., 2017), illustrating the dynamics of Midkine expression after cardiac injury.

\section{The mdka-KO line}

To study Mdka function in heart regeneration, we generated $m d k a-K O$ zebrafish using CRISPR/Cas9 genetic editing. Mdka-KO embryos developed normally without transcriptional deregulation of $m d k b$. Mdka and Mdkb share 68\% amino acid identity (Winkler et al., 2003) and show overlapping expression patterns in embryos, suggesting that $m d k b$ may compensate the loss of $m d k a$ during development. Interestingly, WM-ISH showed that ptn expression was lost in the forebrain of the developing embryo, however, we did not detect behavioural differences in the $m d k a-K O$ larvae or adults, as it was reported for the $M d k-K O$ mouse (Nakamura et al., 1998).

\section{Mdka in heart regeneration}

In the adult heart, we detected $m d k a$ expression only after cardiac injury, suggesting that $m d k a$ deletion might have adverse results in heart regeneration. In our KO model, Mdka was lost in the injured heart without activation of $m d k b$ or $p t n$, whereas, in the Mdk-KO mouse, Ptn is 
upregulated in intact adult hearts (Herradon et al., 2005). Additionally, data from the mouse reveal that there is a level of functional redundancy between Ptn and Mdk, since the double KO develops severe auditory deficiency via loss of a-Tectorin, whereas in the Mdk-KO or Ptn-KO mice, the phenotype is mild (Zou et al., 2006). These observations suggest that functional compensation in the heart from at least $m d k b$ or $p t n$, two molecules that belong to the same gene family, is unlikely.

Cryoinjury of $m d k a-K O$ hearts resulted in incomplete regeneration at $90 \mathrm{dpci}$, the final timepoint of the regeneration process after cryoinjury. The scar was characterized by the persistent presence of collagen. However, the proliferation rate of CM and epicardial cell was not affected. The fact that we detected more collagen in the injury suggests that Mdka is involved in fibrosis, affecting either collagen production and deposition, or ECM clearance. For the former, treatment of mouse hearts with Mdk after MI leads to decreased collagen deposition (Takenaka et al., 2009). However in pulmonary fibrosis, loss of $M d k$ results in decrease collagen and TGF $\beta$ expression in the lungs (Misa et al., 2017). Additionally, Mdk administration in cultured skin fibroblasts increases collagen (type I and III) and hyaluronic acid synthesis in a dose-dependent manner, as well as transcription of TGFb1 (Yamada et al., 1997). Fibroblasts are the main source of ECM [reviewed in (Kendall and Feghali-Bostwick, 2014)] and RNA-seq data shows that $m d k a$ is highly upregulated in epicardial-derived fibroblast in regenerating zebrafish hearts (Sánchez-Iranzo et al., 2018a). Additionally, $m d k a$ is detected in a broad-epicardial manner and epicardial cells secrete fibronectin (Wang et al., 2013). The above suggest that Mdka may be involved in ECM deposition from epicardial cells possible via interaction with TGF $\beta$ signalling.

Another possibility is that Mdka is involved in ECM clearance by regulating MMPs production directly or by affecting immune cells recruitment. Indeed, treatment of skin fibroblasts with Mdk results in increased $m m p 2$ transcription (Yamada et al., 1997) and also Mdk is involved in neutrophils tethering (Weckbach et al., 2014). In the early phase of the inflammatory response, immune cells express MMPs and remodel ECM, that in turn mediates leukocytes recruitment by activation of chemokines [reviewed in (Frangogiannis, 2019)]. Similarly in zebrafish heart regeneration, inhibition of MMPs results in reduction of macrophages and neutrophils infiltration in the injured area (Xu et al., 2018). Although the profile of the ECM changes during regeneration (Garcia-Puig et al., 2019), MMPs such as mmp2, mmp9, mmp13 and mmp14 are upregulated upon injury and most importantly, collagenolytic activity is reported to be active at least until 30dpci, mediating scar tissue degradation (Gamba et al., 2017; Xu et al., 2018). Hence, loss of Mdka may affected ECM clearance, resulting in a collagen-enriched scar.

Furthermore, $m d k a$ deletion may affect vascularization via impaired immune cells infiltration. Delayed immune response in zebrafish heart regeneration is associated with impaired neovascularization and resolution of the scar tissue (Lai et al 2017), whereas long retention of neutrophils in the wound promotes revascularization (Xu et al 2019). Interestingly, we found $m d k a$ on $w t 1 b^{+}$epicardial cells, and $w t 1 b$ is also active in a subset of macrophages (Sanz-Morejón et al., 
2019). These $w t 1 b^{+}$macrophages acquire a pro-regenerative profile and their depletion impairs heart regeneration. However, our results showed that proliferation of epicardial cells was not affected by loss of Mdka, suggesting that Mdka functions in other immune cells. Mdka could affect recruitment of other leukocytes, such as neutrophils (Weckbach et al., 2014). That could occur during the acute inflammatory or later phase of cardiac healing, to promote angiogenesis.

Moreover, Mdka is a cytokine and it is shown to activate PI3-kinase/Akt and ERK1/2 phosphorylation cascades after mouse heart injury (Sumida et al., 2010; Takenaka et al., 2009). In zebrafish heart regeneration, blockage of the MAPK/ERK signalling pathway leads to regeneration arrest and delayed vascularization without inhibition of CM proliferation. Hence, Mdka may be involved in angiogenesis via activation of phosphorylation cascades.

Overall, Mdka implications in zebrafish heart regeneration can be in ECM deposition/degradation directly or via immune cells recruitment, that could also affect revascularization of the regenerating tissue. Future experiment will clarify why the heart of $m d k a-$ $K O$ zebrafish does not regenerate. 


\section{Dissection and in vivo evaluation of $m d k a$ epigenetic landscape}

Mdka during development is expressed in neural tissues including the brain and the neural tube (Schäfer et al., 2005; Winkler et al., 2003). Here, we found that $m d k a$ is not expressed in intact hearts and that is strongly upregulated in injured heart, having dynamic expression in the epicardium. To explain this change in the spatio-temporal activation of $m d k a$ and to identify the minimum regulatory sequences that drive $m d k a$ expression in development and regenerating, we focused on the epigenetic regulation of $m d k a$. For that, we took advantage of available ChIP-seq and ATAC-seq data from embryos and regenerating hearts (Bogdanović et al., 2012; Goldman et al., 2017; Quillien et al., 2017). We assembled the epigenetic landscape of $m d k a$ and investigated in vivo the expression of a GFP reporter driven by the detected cis-regulatory elements.

\section{The $\operatorname{Tg}(e n h-m d k a: G F P)$ reporter}

The first lines we designed, $\operatorname{Tg}(m d k a: G F P)$ and $\operatorname{Tg}($ enh:GFP), expressed GFP in the embryos similarly to $m d k a$ expression. However, we did not detect GFP in regenerating heart that we found strong $m d k a$ expression, nor in injured fin that $m d k a$ is also activated (González Rajal, 2012). Therefore, we combined the $m d k a$ promoter and the large intronic enhancer, enh, in the $\operatorname{Tg}($ enh-mdka:GFP) line that recapitulated $m d k a$ endogenous expression during development, in adult tissues and after heart and fin injury. In particular, GFP was detected in the outer layer of the fore-, mid-, and hindbrain, in and in cells surrounding cranial ganglia resembling mantle cells. Additionally, we found GFP expressing cells adjacent to neural axons and in the neural tube, resembling the localization of radial glia cells, neural precursors cell that in zebrafish are maintained into adulthood (Bernardos and Raymond, 2006). During development, m $d k a$ is expressed in the neural ectoderm (Winkler et al., 2003) that gives rise to the neural tube [reviewed in (Schmidt et al., 2013)]. Hence, the GFP expression analysis and $m d k a$ developmental expression suggest that the GFP is induced in glia cells. Use of established glia markers such as gfap (Kim et al., 2008b) will elucidate this hypothesis.

The neuromasts of the lateral line contain the mechanosensory hair cells surrounded by mantle and supporting cells (Dambly-Chaudière et al., 2003). After loss of hair cells, mantle cell proliferate to contribute to the new neuromasts (Williams and Holder, 2000). Interestingly, $m d k a$ expression is induced in mantle cells after hair cell ablation (Steiner et al., 2014) and Creb1, a TF predicted to bind to mdka-enh sequences, is expressed in mantle cells (Jiang et al., 2014). Additionally, Creb1 is active in the adult brain including the ventricular zone of the dorsal telencephalon where radial glia is located, and coinciding with the location where we also found GFP expression (Dworkin et al., 2007; März et al., 2010). Collectively, all the TF predicted to bind to the mdka- enh regions are expressed in the CNS and in the retina similarly to $m d k a$, thus explaining the restricted pattern of GFP expression in the CNS (Chen et al., 2012; Erickson et al., 
2010; Hammond and Udvadia, 2010; Rojas et al., 2007; Tallafuss et al., 2006; Thisse, 2001; Webb et al., 2011).

The $T g(e n h-m d k a: G F P)$ line also expressed GFP in regenerating fins and hearts. The TFbinding site analysis revealed that Hifla and Rxra had binding sites in the mdka-enh, sequence and thus, both may activate GFP expression upon injury. Interestingly, although the enh region alone had predicted binding sites for Hifla and Rxra, it was not sufficient to drive GFP expression after injury. This implies that the combination of this enhancer with the endogenous promoter was necessary to drive expression, since neither $\operatorname{Tg}(e n h: G F P)$ nor $T g(m d k a: G F P)$ expressed GFP upon injury. Hypoxia is detected in the epicardium and in the damaged area during zebrafish heart regeneration (Jopling et al., 2012) and the promoter of mammalian Mdk contains HIF1-responsve elements (Reynolds et al., 2004). Therefore, it was possible that GFP could be expressed in response to hypoxia in regenerating hearts. Moreover, $M d k$ was identified after a screen for RA-responsive gene (Kadomatsu et al., 1988), and it was found that contains RA-responsive elements (Matsubara et al., 1994). RA signalling is important for heart regeneration and it is active in epicardial cells (Kikuchi et al., 2011b), suggesting that RA could be upstream of $m d k a$. To test these hypotheses, further functional experiment will be the exposure of transgenic larvae to hypoxic conditions and/or use of the $\operatorname{Tg}($ hsp70l:cyp26al) line that expresses the RA-degrading Cyp26 enzyme after heatshock (Kikuchi et al., 2011b).

\section{The $\operatorname{Tg}(\operatorname{Reg}: G F P)$ transgenic line}

The $\operatorname{Tg}(\operatorname{Reg}: G F P)$ line was generated because a short intronic region was found to be active in regenerating hearts (Goldman et al., 2017). Intriguingly, Reg was sufficient to drive GFP expression in embryos similar to $m d k a$ and to $T g(e n h-m d k a: G F P)$. We detected restricted expression of GFP in the dorsal hindbrain, in cells surrounding nerves, as well as in the lateral line. In the cranial ganglia, $\mathrm{GFP}^{+}$cells were perineural of neuromasts' branches, adjacent to the neuromasts. Moreover, $\mathrm{GFP}^{+}$cells were adjacent to neural axons expanding from the spinal tube. These results suggest that the GFP expressing cells could be glial cells, similarly to the $\operatorname{Tg}(e n h$ $m d k a: G F P)$ line.

In $\operatorname{Tg}(\operatorname{Reg}: G F P)$ line, GFP expression was induced after heart injury but not in epicardial cells, where $m d k a$ was detected. Interestingly, binding sites for Nkx5-5 and Mef2A (Hammond and Udvadia, 2010; Zhang et al., 2018) were predicted to exist in the Reg sequences implying that the GFP expression in the adult heart is CM-specific.

\section{The $T g(e n h a 4: G F P)$ and $T g(e n h a 5: G F P)$ transgenic lines}

The $T g(e n h a 4: G F P)$ expression in embryos was partially similar to $m d k a$, with some GFP detected in the brain and the retina. The interesting observation was that the GFP expression shifted from endocardia cells and CM, to valve-specific during development. Additionally, we observed 
that GFP was strongly expressed in the vasculature of the regenerating fin. Such a peculiar pattern implies that enha4 is a "broad enhancer" able to interact along time with different TF. Indeed, analysis of the enha4 sequence predicted binding sites for neural specific TF as Sox19 and Max (Chen et al., 2012; Okuda et al., 2006; Webb et al., 2011), cardiac TF as Nkx2-5 and Fos-Jun (Thisse, C., and Thisse, 2004; Thisse, 2001; Zhang et al., 2018) and endothelial TF as Fli1 (Thompson et al., 1998). Furthermore, the dynamics of the GFP expression in embryonic heart and the TF analysis indicate that the detected GFP expression in the injured heart could be under the control of multiply TFs and in different type of cells.

The $\operatorname{Tg}($ enha5:GFP) line had strong expression in $\mathrm{CM}$ of the developing heart, and the expression persisted in adults. This observation is consistent with the results of our in silico analysis, that predicted binding sites for Nkx2-5, Mef2 and Nfatc2 (Hammond and Udvadia, 2010; Zhang et al., 2018) in the enha5 sequence. Interestingly, $\operatorname{Tg}($ enha5:GFP) was the only line in which the pectoral fin was strongly labelled, as we detected in $m d k a$ WM-ISH analysis. That implies that enha5 is responsible for $m d k a$ expression in the pectoral fin.

\section{Conclusions of cis-regulatory elements study}

Analysis of the cis-regulatory sequences can be helpful for understanding how the expression pattern and function of genes is regulated in development and disease (Franke and Gómez-Skarmeta, 2018). Our in vivo evaluation showed that it is possible to identify the minimum regulatory sequences that can be used as drivers in transgenic reporter or overexpression lines, designed to study the function of genes with dynamic and widespread expression (Yuan et al., 2018). It can be an alternative to BAC (Bacterial Artificial Chromosome) transgenesis, especially for genes without appropriate BAC (Bussmann and Schulte-Merker, 2011). Additionally, this approach may reveal injury-specific regulatory sequences. Such information is very valuable, since injury-active regions can be deleted and prevent the activation of genes upon injury and not during development, overcoming possible genomic compensation (Zhu et al., 2018). 
Caveolin-1 is the main structural protein of caveolae, small membrane invaginations involved in signal transduction and in mechanoprotection (Gervásio et al., 2011; Sinha et al., 2011; Sowa et al., 2001). Here, we investigated the importance of Cav1 and caveolae in heart homeostasis and regeneration using cryoinjury. We found that Cav1 has a strong expression upon injury but deletion of Cav1/caveolae did not affect heart regeneration. Still, loss of Cav1/caveolae results in an increased in cardiac stiffness and affects cardiac function.

\section{Cav1 expression in intact and injured heart}

Our data show that Cav1 had a broad and robust expression in intact hearts, detected in the endothelium, endocardium and epicardium. The expression of Cav1 was upregulated after injury and strongly expressed 7dpci in epicardial cells that have covered the damaged tissue (GonzálezRosa et al., 2012). We also found Cav1 in the endocardium invading the injured area and creating a barrier between the proliferating CM and the damaged tissue (Kikuchi et al., 2011c; Münch et al., 2017). Both, epicardial and endocardial cells get activated early after the cardiac insult contributing to the regeneration process (Gonzalez-Rosa et al., 2011; Kikuchi et al., 2011c; Münch et al., 2017; Schnabel et al., 2011).

\section{Generation of the $\operatorname{cav} 1-K O$ zebrafish}

To examine the role of Cav1 in the cardiac tissue we generated zebrafish strains carrying cavl genetic mutations that hypothetically would encode proteins lacking the carboxy tail, important for Cav1 trafficking and caveolae formation (Ren, 2004; Schlegel and Lisanti, 2000; Song et al., 1997). These mutant proteins were predicted to be shorter with novel amino acids in the C-terminal half. Here, we have focused on two cavl mutant alleles, X136 and X166, that affected differently cavl transcription. In X136, cavl transcription was decreased possibly due to non-sense mediated decay (Frischmeyer et al., 2002), in contrast to X166 in which cav1 transcription was unaffected. Nevertheless, both X136 and X166 genetic mutations led to loss of Cav1 protein expression resulting in cav1-KO. In the case of X136, the decrease of the transcript via non-sense mediated decay could explain loss of the protein. However, in X166, loss of the protein could possibly occur through degradation, since mutations in C-terminal domain often result in conformational changes altering protein's solubility, leading to degradation (Elstak et al., 2012). Interestingly, cav2 was upregulated in both cases suggesting that the compensatory mechanisms were activated (El-Brolosy and Stainier, 2017). Since Cav1 is indispensable for caveolae formation, we examined if this was the case in our cavl-KO zebrafish models. TEM analysis showed that caveolae were completely lost in both X136 and X166 hearts, whereas they were abundant in WT hearts. Thus, X136 and X166 led to loss of Cav1 and consequently of caveolae. Furthermore, loss of Cav1 also affected Cavin1 protein expression, although transcriptionally appeared unaffected. The cavl-KO hearts lacked Cavin1 in the valves and the 
overall expression was decreased, as observed in immunofluorescence and WB analysis, similarly to what has been reported in the Cav1-KO mouse (Hansen et al., 2013; Hill et al., 2008; Taniguchi et al., 2016). Furthermore, we found that cav2 was upregulated transcriptionally. However, further analysis of Cav2 protein expression in the cav1-KO hearts was not carried out because experiments in the Cav1-KO models show that deletion of Cav1 results in Cav2 loss (Drab et al., 2001; Park et al., 2003; Razani et al., 2001a).

\section{Cav1 is not required for adult zebrafish heart regeneration after cryoinjury}

To investigate the effect of Cav1/caveolae loss in cardiac regeneration we have used the cryoinjury model that bears the importance of scar tissue formation that gradually resolves leading to complete tissue regeneration at 90dpci (Chablais and Jaźwińska, 2012; Gonzalez-Rosa et al., 2011; Schnabel et al., 2011). We monitored the regeneration process every 30-days and we found that the injury size in the cav1-KO hearts was similar (30-and 60dpci) to the WT, reaching complete cardiac regeneration at $90 \mathrm{dpci}$. This was an unexpected result, since it has been reported that ventricular resection in a cav1-KO zebrafish model results in increased ECM deposition, leading to arrest of heart regeneration 30dpi (Cao et al., 2016). The zebrafish cardiac regeneration model previously used (ventricular resection) has a significant difference compared to cryoinjury. Cryoinjury leads to formation of a transient scar tissue, whereas in the ventricular resection model, the fibrotic scar is minimal. Additionally, there is evidence suggesting that these two cardiac injury methods result in different regenerative responses (Vivien et al., 2019). Thus, it is possible that loss of Cav1 impacts differently on the regeneration process, depending on the injury model. Importantly, the regeneration process in the resection model completes within 60-days, which is the final point of full cardiac recovery (Poss et al., 2002) and the effect of Cav1 inactivation was examined only until 30-days after ventricular resection (Cao et al., 2016), when the regeneration process is not fully completed, and subtle differences in the regeneration dynamics between WT and cav1-KO hearts could be interpreted as a loss of regenerative ability in cavl-KO.

\section{Fibrosis in the cavl-KO heart}

Caveolin-1 negatively regulates TGF $\beta$ signalling (Razani et al., 2001b) and loss of Cav1 leads to increased ECM deposition after injury (Miyasato et al., 2011; Shivshankar et al., 2014). However, the quantity of collagen inside the damaged tissue was not affected, nor TGF $\beta$ pathway activation. Additionally, intact cavl-KO hearts were not characterized by interstitial fibrosis as has been reported in the mouse Cav1-KO models (Cohen et al., 2003; Drab et al., 2001; Murata et al., 2007; Park et al., 2003). Moreover, Cav1 was detected in the epicardium covering the injured area and in endocardial cells invading the damaged tissue. Upon injury, the epicardial cells are comprised of fibroblast and macrophages that contribute to fibrosis (Sánchez-Iranzo et al., 2018a; Sanz-Morejón et al., 2019), and loss of Cav1 has been associated with increased fibrosis and 
macrophages infiltration after cardiac injury (Miyasato et al., 2011; Shivshankar et al., 2014). However, the proliferation rate of epicardial cells did not change, indicating that these two cell types are unaffected in our Cav1-deficient hearts. In addition, endocardial cells have been associated to collagen (Münch et al., 2017) and Cav1 was completely lost in these cells. However, endocardial cell abundance was similar between WT and X136 hearts, suggesting that their proliferation and migration was unaffected. In addition, TGF $\beta$ signalling activation during regeneration appeared to be unaffected, explaining the normal ECM deposition upon cryoinjury. Collectively, these findings indicate that loss of Caveolin-1 and caveolae does not lead to deregulation of fibrosis in intact, or in cryoinjured hearts. It is well documented, however, that Cav1 interacts and regulates TGF $\beta$ signalling so that the relatively normal ECM deposition in our cav1$K O$ suggests that there is a compensatory mechanism buffering Cav1 loss (El-Brolosy et al., 2019).

\section{Cav1 is transiently required for $\mathrm{CM}$ proliferation during regeneration}

Interestingly and in agreement with a previous report, CM proliferation was significantly decreased 7dpci (Cao et al., 2016). However, that was not reflected in defective or even delayed regeneration. Indeed, CM proliferation rate at $14 \mathrm{dpci}$ was similar to the control, so that $\mathrm{CM}$ were able to increase in numbers, populate the damaged area and contribute to normal heart regeneration. A variety of approaches have showed that CAV1 is weakly expressed in mammalian CM (Cho et al., 2010; Chow et al., 2007; Patel et al., 2007; Robenek et al., 2008b). In zebrafish, available RNAseq data from gata ${ }^{+} \mathrm{CM}$ of injured hearts and cortical $\mathrm{CM}$ from intact hearts show low cav1 expression (Ben-Yair et al., 2019; Sánchez-Iranzo et al., 2018b). Furthermore, the stiffness of ventricular myocardium is increased upon injury only in the border zone between healthy and damaged tissue, where the proliferative CM are detected, and is normalized between 14-35dpci ( $\mathrm{Yu}$ et al., 2018). Studies from mouse embryonic fibroblast show that loss of Cav1 leads to increased Yap phosphorylation in response to stiffer environment (Moreno-Vicente et al., 2018), and hence to Yap cytoplasmic detention and transcriptional arrest of Yap target genes. YAP nuclear localization is important for mouse embryonic CMs proliferation and heart growth, as well as adult CMs renewal after injury (Heallen et al., 2013; Lin et al., 2014; Von Gise et al., 2012; Xin et al., 2013). In our model, caveolae depletion may leads to increased Yap phosphorylation in response of the stiffer border zone which may explain the decrease in CM proliferation that is normalized by 14dpci. Further investigation is required to test this hypothesis and to examine other signalling molecules that are involved in CM proliferation during zebrafish regeneration.

\section{Cav1 is necessary for proper heart function and cardiac cell stiffness}

Cavl-deficient mouse shows abnormal cardiac remodelling, including dilation and hypertrophy of the chambers (Cohen et al., 2003; Drab et al., 2001; Murata et al., 2007; Park et al., 2003). No such phenotypes were detected in our cavl-KO zebrafish line, since neither the size of 
the ventricle was altered, nor fibrosis was observed in the cavl-KO hearts. Furthermore, in Cav1null mouse heart, the systolic and diastolic functions of the heart are affected (Cohen et al., 2003; Park et al., 2003; Wunderlich et al., 2006; Zhao et al., 2002). Accordingly, our X136 line showed reduced cardiac performance, as indicated by the reduced EF that was accompanied by a lower heart rate. Since we did not detect cardiac fibrosis, the switch in cardiac elasticity could provide explanation for the cardiac dysfunction of cavl-KO hearts. Membrane invaginations such as caveolae, provide the necessary stretch capacity for cells to buffer the impact of mechanical forces (Gervásio et al., 2011; Sinha et al., 2011). Our AFM analysis revealed that cavl-KO hearts were significantly stiffer than the WT. The elasticity of the cardiac tissue was decreased almost twofolds in the caveolae-deprived hearts. That significant switch in the biomechanical properties of the cardiac tissue undoubtedly was caused by loss of Cav1 expression. The sensitivity of AFM allows us to conclude that the measurements represent the stiffness of both the epicardial cells and the underlying cortical myocytes. Here, we found that Cav1 was strongly expressed in the epicardium of intact hearts and that its expression was lost in X136 hearts. Additionally, RNA-seq data show that Cav1 is moderately expressed in cortical CM, but significantly higher than in trabecular CM (Sánchez-Iranzo et al., 2018b). Caveolae are required for smooth muscle cells contractility (Gosens et al., 2011; Halayko et al., 2008) and cell contraction results in changes in membrane tension that caveolae absorb (Gervásio et al., 2011; Sinha et al., 2011). In addition, Cav1 and caveolae regulate RhoA activation (a GTPase protein that regulates cytoskeleton) that in turn regulates actomyosin contractility (Budnar et al., 2019; Grande-García et al., 2007; Peng et al., 2007). Hence, all these data suggest that loss of Cav1/caveolae impaired cell elasticity and/or contractility and therefore, pumping efficiency, which could explain the drop in cardiac performance.

Lastly, Cav1 regulates eNOS by direct binding (García-Cardeña et al., 1996) and the availability of nitric oxide (NO) in the endothelium that mediates vessel relaxation (Palmer et al., 1987). Additionally, endothelial-specific Cav1 reconstruction in Cav1-KO mice rescues the cardiac and pulmonary phenotypes, highlighting the importance of Cav1-eNOS interaction (Murata et al., 2007). Here, we report strong expression of Cav1 in endothelium that is lost in our cav1-KO model. Thus, we cannot exclude the possibility that increased eNOS activity could also affect the cavl-KO cardiac performance, and that should be addressed in future studies. 
Conclusions 


\section{Conclusions}

1. Loss of Mdka results in arrest of heart regeneration and increased collagen deposition in the injured area.

2. The promoter of $m d k a$ in combination with the intronic enhancers can recapitulate $m d k a$ endogenous expression during development, in adult tissues and in heart and fin regeneration.

3. Loss of Cav1 and caveolae do not affect heart regeneration upon cryoinjury.

4. Caveolae and Cav1 loss do not lead to neither cardiac remodelling nor fibrosis in intact or injured hearts.

5. Loss of Cav1 and caveolae increases the stiffness of the epicardial cells and cortical cardiomyocytes, hence altering the mechanical properties of the heart.

6. Cav1 is important for normal adult cardiac function. 


\section{Conclusiones}

1. La deleción de $m d k a$ produce un bloqueo en la regeneración cardiaca y un aumento de la deposición de colágeno en el área dañada.

2. La mínima secuencia regulatoria en cis de $m d k a$ con actividad enhancer y capaz de recapitular el patrón endógeno de expresión de $m d k a$ durante el desarrollo y la regeneración del corazón y la aleta, se ha encontrado en un intron.

3. La pérdida de cav1 y de las caveolas no afecta a la regeneración cardiaca del pez cebra tras criolesión.

4. La eliminación de las caveolas y de Cav1 no conducen ni a la remodelación cardiaca ni a fibrosis en corazones intactos o dañados.

5. La pérdida de Cav1 y de caveolas induce un aumento en la rigidez de las células epicárdicas y de los cardiomiocitos corticales, lo que altera las propiedades mecánicas del corazón.

6. Cav1 es importante para la función cardíaca normal del adulto. 
Bibliography 
Akimenko, M. A., Johnson, S. L., Westerfield, M. and Ekker, M. (1995). Differential induction of four msx homeobox genes during fin development and regeneration in zebrafish. Development.

Alford, R. F., Koehler Leman, J., Weitzner, B. D., Duran, A. M., Tilley, D. C., Elazar, A. and Gray, J. J. (2015). An Integrated Framework Advancing Membrane Protein Modeling and Design. PLoS Comput. Biol.

Ann, F, R., Hsu, P. D., Wright, J., Agarwala, V., Scott, D. A. and Zhang, F. (2013). Genome engineering using the CRISPR-Cas9. Nat Protoc.

Austin, E. D., Ma, L., LeDuc, C., Rosenzweig, E. B., Borczuk, A., Phillips, J. A., Palomero, T., Sumazin, P., Kim, H. R., Talati, M. H., et al. (2012). Whole exome sequencing to identify a novel gene (Caveolin-1) associated with human pulmonary arterial hypertension. Circ. Cardiovasc. Genet.

Bader, D. and Oberpriller, J. O. (1978). Repair and reorganization of minced cardiac muscle in the adult newt (Notophthalmus viridescens). J. Morphol.

Bai, L., Deng, X., Li, J., Wang, M., Li, Q., An, W., A, D. and Cong, Y. S. (2011). Regulation of cellular senescence by the essential caveolar component PTRF/Cavin-1. Cell Res.

Banno, H., Takei, Y., Muramatsu, T., Komori, K. and Kadomatsu, K. (2006). Controlled release of small interfering RNA targeting midkine attenuates intimal hyperplasia in vein grafts. J. Vasc. Surg.

Barth, P., Wallner, B. and Baker, D. (2009). Prediction of membrane protein structures with complex topologies using limited constraints. Proc. Natl. Acad. Sci.

Bastiani, M., Liu, L., Hill, M. M., Jedrychowski, M. P., Nixon, S. J., Lo, H. P., Abankwa, D., Luetterforst, R., Fernandez-Rojo, M., Breen, M. R., et al. (2009). MURC/Cavin-4 and cavin family members form tissue-specific caveolar complexes. J. Cell Biol.

Becker, T., Wullimann, M. F., Becker, C. G., Bernhardt, R. R. and Schachner, M. (1997). Axonal regrowth after spinal cord transection in adult zebrafish. J. Comp. Neurol.

Bednarek, D., González-Rosa, J. M., Guzmán-Martínez, G., Gutiérrez-Gutiérrez, Ó., Aguado, T., Sánchez-Ferrer, C., Marques, I. J., Galardi-Castilla, M., de Diego, I., Gómez, M. J., et al. (2015). Telomerase Is Essential for Zebrafish Heart Regeneration. Cell Rep.

Ben-Yair, R., Butty, V. L., Busby, M., Qiu, Y., Levine, S. S., Goren, A., Boyer, L. A., Burns, C. G. and Burns, C. E. (2019). H3K27me3-mediated silencing of structural genes is required for zebrafish heart regeneration. Development.

Benjamin, E. J., Muntner, P., Alonso, A., Bittencourt, M. S., Callaway, C. W., Carson, A. P., Chamberlain, A. M., Chang, A. R., Cheng, S., Das, S. R., et al. (2019). Heart Disease and Stroke Statistics-2019 Update: A Report From the American Heart Association. Circulation.

Bernardos, R. L. and Raymond, P. A. (2006). GFAP transgenic zebrafish. Gene Expr. Patterns. 
Bessa, J., Tena, J. J., De La Calle-Mustienes, E., Fernández-Miñán, A., Naranjo, S., Fernández, A., Montoliu, L., Akalin, A., Lenhard, B., Casares, F., et al. (2009). Zebrafish Enhancer Detection (ZED) vector: A new tool to facilitate transgenesis and the functional analysis of cis-regulatory regions in zebrafish. Dev. Dyn.

Bogdanović, O., Fernandez-Miñán, A., Tena, J. J., De La Calle-Mustienes, E., Hidalgo, C., Van Kruysbergen, I., Van Heeringen, S. J., Veenstra, G. J. C. and Gómez-Skarmeta, J. L. (2012). Dynamics of enhancer chromatin signatures mark the transition from pluripotency to cell specification during embryogenesis. Genome Res.

Budnar, S., Husain, K. B., Gomez, G. A., Naghibosadat, M., Varma, A., Verma, S., Hamilton, N. A., Morris, R. G. and Yap, A. S. (2019). Anillin Promotes Cell Contractility by Cyclic Resetting of RhoA Residence Kinetics. Dev. Cell.

Bussmann, J. and Schulte-Merker, S. (2011). Rapid BAC selection for tol2-mediated transgenesis in zebrafish. Development.

Calinescu, A. A., Vihtelic, T. S., Hyde, D. R. and Hitchcock, P. F. (2009a). Cellular expression of midkine-a and midkine-b during retinal development and photoreceptor regeneration in zebrafish. J. Comp. Neurol.

Calinescu, A. A., Raymond, P. A. and Hitchcock, P. F. (2009b). Midkine expression is regulated by the circadian clock in the retina of the zebrafish. Vis. Neurosci.

Cao, H., Alston, L., Ruschman, J. and Hegele, R. A. (2008). Heterozygous CAV1 frameshift mutations (MIM 601047) in patients with atypical partial lipodystrophy and hypertriglyceridemia. Lipids Health Dis.

Cao, J., Navis, A., Cox, B. D., Dickson, A. L., Gemberling, M., Karra, R., Bagnat, M. and Poss, K. D. (2016). Single epicardial cell transcriptome sequencing identifies Caveolin 1 as an essential factor in zebrafish heart regeneration. Development.

Chablais, F. and Jazwinska, A. (2012). The regenerative capacity of the zebrafish heart is dependent on TGF signaling. Development.

Chablais, F. and Jaźwińska, A. (2012). Induction of Myocardial Infarction in Adult Zebrafish Using Cryoinjury. J. Vis. Exp.

Chablais, F., Veit, J., Rainer, G. and Jawiska, A. (2011). The zebrafish heart regenerates after cryoinjury-induced myocardial infarction. BMC Dev. Biol.

Chang, M. H., Huang, C. J., Hwang, S. P. L., Lu, I. C., Lin, C. M., Kuo, T. F. and Chou, C. M. (2004). Zebrafish heparin-binding neurotrophic factor enhances neurite outgrowth during its development. Biochem. Biophys. Res. Commun.

Chen, Y. Y., Harris, M. P., Levesque, M. P., Nüsslein-Volhard, C. and Sonawane, M. (2012). Heterogeneity across the dorso-ventral axis in zebrafish EVL is regulated by a novel module consisting of sox, snailla and max genes. Mech. Dev.

Cheng, J. P. X., Mendoza-Topaz, C., Howard, G., Chadwick, J., Shvets, E., Cowburn, A. S., 
Dunmore, B. J., Crosby, A., Morrell, N. W. and Nichols, B. J. (2015). Caveolae protect endothelial cells from membrane rupture during increased cardiac output. J. Cell Biol.

Cherian, A. V., Fukuda, R., Augustine, S. M., Maischein, H. M. and Stainier, D. Y. R. (2016). N-cadherin relocalization during cardiac trabeculation. Proc. Natl. Acad. Sci. U. S. A.

Cho, W. J., Chow, A. K., Schulz, R. and Daniel, E. E. (2010). Caveolin-1 exists and may function in cardiomyocytes. Can. J. Physiol. Pharmacol.

Chow, A. K., Cena, J., El-Yazbi, A. F., Crawford, B. D., Holt, A., Cho, W. J., Daniel, E. E. and Schulz, R. (2007). Caveolin-1 inhibits matrix metalloproteinase-2 activity in the heart. J. Mol. Cell. Cardiol.

Cohen, A. W., Park, D. S., Woodman, S. E., Williams, T. M., Chandra, M., Shirani, J., De Souza, A. P., Kitsis, R. N., Russell, R. G., Weiss, L. M., et al. (2003). Caveolin-1 null mice develop cardiac hypertrophy with hyperactivation of p42/44 MAP kinase in cardiac fibroblasts. Am. J. Physiol. - Cell Physiol. 284,

Cong, L., Ran, F. A., Cox, D., Lin, S., Barretto, R., Habib, N., Hsu, P. D., Wu, X., Jiang, W., Marraffini, L. A., et al. (2013). Multiplex genome engineering using CRISPR/Cas systems. Science (80-. ).

Conway, P., Tyka, M. D., DiMaio, F., Konerding, D. E. and Baker, D. (2014). Relaxation of backbone bond geometry improves protein energy landscape modeling. Protein Sci.

Couet, J., Li, S., Okamoto, T., Ikezu, T. and Lisanti, M. P. (1997). Identification of peptide and protein ligands for the caveolin- scaffolding domain. Implications for the interaction of caveolin with caveolae-associated proteins. J. Biol. Chem.

Covassin, L. D., Siekmann, A. F., Kacergis, M. C., Laver, E., Moore, J. C., Villefranc, J. A., Weinstein, B. M. and Lawson, N. D. (2009). A genetic screen for vascular mutants in zebrafish reveals dynamic roles for Vegf/Plcg1 signaling during artery development. Dev. Biol.

Dambly-Chaudière, C., Sapède, D., Soubiran, F., Decorde, K., Gompel, N. and Ghysen, A. (2003). The lateral line of zebrafish: A model system for the analysis of morphogenesis and neural development in vertebrates. Biol. Cell.

Das, B. C., Thapa, P., Karki, R., Das, S., Mahapatra, S., Liu, T. C., Torregroza, I., Wallace, D. P., Kambhampati, S., Van Veldhuizen, P., et al. (2014). Retinoic acid signaling pathways in development and diseases. Bioorganic Med. Chem.

De Preux Charles, A. S., Bise, T., Baier, F., Marro, J. and Jaźwińska, A. (2016). Distinct effects of inflammation on preconditioning and regeneration of the adult zebrafish heart. Open Biol.

Dorighi, K. M., Swigut, T., Henriques, T., Bhanu, N. V., Scruggs, B. S., Nady, N., Still, C. D., Garcia, B. A., Adelman, K. and Wysocka, J. (2017). M113 and Ml14 Facilitate Enhancer RNA Synthesis and Transcription from Promoters Independently of H3K4 Monomethylation. Mol. Cell. 
Drab, M., Verkade, P., Elger, M., Kasper, M., Lohn, M., Lauterbach, B., Menne, J., Lindschau, C., Mende, F., Luft, F. C., et al. (2001). Loss of caveolae, vascular dysfunction, and pulmonary defects in caveolin-1 gene-disrupted mice. Science (80-. ).

Dworkin, S., Heath, J. K., deJong-Curtain, T. A., Hogan, B. M., Lieschke, G. J., Malaterre, J., Ramsay, R. G. and Mantamadiotis, T. (2007). CREB activity modulates neural cell proliferation, midbrain-hindbrain organization and patterning in zebrafish. Dev. Biol.

El-Brolosy, M. A. and Stainier, D. Y. R. (2017). Genetic compensation: A phenomenon in search of mechanisms. PLoS Genet.

El-Brolosy, M. A., Kontarakis, Z., Rossi, A., Kuenne, C., Günther, S., Fukuda, N., Kikhi, K., Boezio, G. L. M., Takacs, C. M., Lai, S. L., et al. (2019). Genetic compensation triggered by mutant mRNA degradation. Nature.

Elstak, E. D., Te Loo, M., Tesselaar, K., Van Kerkhof, P., Loeffen, J., Grivas, D., Hennekam, E., Boelens, J. J., Hoogerbrugge, P. M., Van der Sluijs, P., et al. (2012). A novel Dutch mutation in UNC13D reveals an essential role of the C2B domain in munc13-4 function. Pediatr. Blood Cancer.

Erickson, T., French, C. R. and Waskiewicz, A. J. (2010). Meis1 specifies positional information in the retina and tectum to organize the zebrafish visual system. Neural Dev.

Fan, Q. W., Muramatsu, T. and Kadomatsu, K. (2000). Distinct expression of midkine and pleiotrophin in the spinal cord and placental tissues during early mouse development. Dev. Growth Differ.

Fang, P. K., Solomon, K. R., Zhuang, L., Qi, M., McKee, M., Freeman, M. R. and Yelick, P. C. (2006). Caveolin-1 $\alpha$ and $-1 \beta$ perform nonredundant roles in early vertebrate development. Am. J. Pathol.

Fang, Y., Gupta, V., Karra, R., Holdway, J. E., Kikuchi, K. and Poss, K. D. (2013). Translational profiling of cardiomyocytes identifies an early Jak1/Stat3 injury response required for zebrafish heart regeneration. Proc. Natl. Acad. Sci. U. S. A.

Fielding, C. J. and Fielding, P. E. (2004). Membrane cholesterol and the regulation of signal transduction. In Biochemical Society Transactions, .

Flinn, M. A., Jeffery, B. E., O'Meara, C. C. and Link, B. A. (2019). Yap is required for scar formation but not myocyte proliferation during heart regeneration in zebrafish. Cardiovasc. Res.

Fra, A. M., Williamson, E., Simons, K. and Parton, R. G. (1995). De novo formation of caveolae in lymphocytes by expression of VIP21-caveolin. Proc. Natl. Acad. Sci.

Frangogiannis, N. G. (2019). The Extracellular Matrix in Ischemic and Nonischemic Heart Failure. Circ. Res.

Franke, M. and Gómez-Skarmeta, J. L. (2018). An evolutionary perspective of regulatory landscape dynamics in development and disease. Curr. Opin. Cell Biol. 
Frischmeyer, P. A., Van Hoof, A., O'Donnell, K., Guerrerio, A. L., Parker, R. and Dietz, H. C. (2002). An mRNA surveillance mechanism that eliminates transcripts lacking termination codons. Science (80-. ).

Fukui, S., Kitagawa-Sakakida, S., Kawamata, S., Matsumiya, G., Kawaguchi, N., Matsuura, N. and Sawa, Y. (2008). Therapeutic Effect of Midkine on Cardiac Remodeling in Infarcted Rat Hearts. Ann. Thorac. Surg.

Galbiati, F., Engelman, J. A., Volonte, D., Zhang, X. L., Minetti, C., Li, M., Hou, H., Kneitz, B., Edelmann, W. and Lisanti, M. P. (2001). Caveolin-3 Null Mice Show a Loss of Caveolae, Changes in the Microdomain Distribution of the Dystrophin-Glycoprotein Complex, and T-tubule Abnormalities. J. Biol. Chem.

Gamba, L., Amin-Javaheri, A., Kim, J., Warburton, D. and Lien, C.-L. (2017). Collagenolytic Activity Is Associated with Scar Resolution in Zebrafish Hearts after Cryoinjury. $J$. Cardiovasc. Dev. Dis.

García-Cardeña, G., Fan, R., Stern, D. F., Liu, J. and Sessa, W. C. (1996). Endothelial nitric oxide synthase is regulated by tyrosine phosphorylation and interacts with caveolin-1. J. Biol. Chem.

Garcia-Puig, A., Mosquera, J. L., Jiménez-Delgado, S., García-Pastor, C., Jorba, I., Navajas, D., Canals, F. and Raya, A. (2019). Proteomics Analysis of Extracellular Matrix Remodeling During Zebrafish Heart Regeneration. Mol. Cell. Proteomics.

Garcia, J., Bagwell, J., Njaine, B., Norman, J., Levic, D. S., Wopat, S., Miller, S. E., Liu, X., Locasale, J. W., Stainier, D. Y. R., et al. (2017). Sheath Cell Invasion and Transdifferentiation Repair Mechanical Damage Caused by Loss of Caveolae in the Zebrafish Notochord. Curr. Biol.

Gemberling, M., Karra, R., Dickson, A. L. and Poss, K. D. (2015). Nrg1 is an injury-induced cardiomyocyte mitogen for the endogenous heart regeneration program in zebrafish. Elife.

Gervásio, O. L., Phillips, W. D., Cole, L. and Allen, D. G. (2011). Caveolae respond to cell stretch and contribute to stretch-induced signaling. J. Cell Sci.

Goldman, J. A., Kuzu, G., Lee, N., Karasik, J., Gemberling, M., Foglia, M. J., Karra, R., Dickson, A. L., Sun, F., Tolstorukov, M. Y., et al. (2017). Resolving Heart Regeneration by Replacement Histone Profiling. Dev. Cell.

Gonzalez-Rosa, J. M., Martin, V., Peralta, M., Torres, M. and Mercader, N. (2011). Extensive scar formation and regression during heart regeneration after cryoinjury in zebrafish. Development.

González-Rosa, J. M. and Mercader, N. (2012). Cryoinjury as a myocardial infarction model for the study of cardiac regeneration in the zebrafish. Nat. Protoc.

González-Rosa, J. M., Peralta, M. and Mercader, N. (2012). Pan-epicardial lineage tracing reveals that epicardium derived cells give rise to myofibroblasts and perivascular cells during 
zebrafish heart regeneration. Dev. Biol.

González-Rosa, J. M., Guzmán-Martínez, G., Marques, I. J., Sánchez-Iranzo, H., JiménezBorreguero, L. J. and Mercader, N. (2014). Use of echocardiography reveals reestablishment of ventricular pumping efficiency and partial ventricular wall motion recovery upon ventricular cryoinjury in the Zebrafish. PLoS One.

González Rajal, Á. (2012). "Identificación y validación funcional de nuevos genes implicados en la regeneración de la aleta y del corazón del pez cebra" (Unpublished doctoral dissertation).

Gosens, R., Stelmack, G. L., Bos, S. T., Dueck, G., Mutawe, M. M., Schaafsma, D., Unruh, H., Gerthoffer, W. T., Zaagsma, J., Meurs, H., et al. (2011). Caveolin-1 is required for contractile phenotype expression by airway smooth muscle cells. J. Cell. Mol. Med.

Gramage, E., D'Cruz, T., Taylor, S., Thummel, R. and Hitchcock, P. F. (2015). Midkine-a protein localization in the developing and adult retina of the zebrafish and its function during photoreceptor regeneration. PLoS One.

Grande-García, A., Echarri, A., De Rooij, J., Alderson, N. B., Waterman-Storer, C. M., Valdivielso, J. M. and Del Pozo, M. A. (2007). Caveolin-1 regulates cell polarization and directional migration through Src kinase and Rho GTPases. J. Cell Biol.

Grant, C. E., Bailey, T. L. and Noble, W. S. (2011). FIMO: Scanning for occurrences of a given motif. Bioinformatics.

Gupta, V. and Poss, K. D. (2012). Clonally dominant cardiomyocytes direct heart morphogenesis. Nature.

Haffner-Luntzer, M., Heilmann, A., Rapp, A. E., Roessler, R., Schinke, T., Amling, M., Ignatius, A. and Liedert, A. (2016). Antagonizing midkine accelerates fracture healing in mice by enhanced bone formation in the fracture callus. Br. J. Pharmacol.

Halayko, A. J., Tran, T. and Gosens, R. (2008). Phenotype and functional plasticity of airway smooth muscle: Role of caveolae and caveolins. Proc. Am. Thorac. Soc.

Halpern, M. E., Hatta, K., Amacher, S. L., Talbot, W. S., Yan, Y. L., Thisse, B., Thisse, C., Postlethwait, J. H. and Kimmel, C. B. (1997). Genetic interactions in zebrafish midline development. Dev. Biol.

Hammond, D. R. and Udvadia, A. J. (2010). Cabin1 expression suggests roles in neuronal development. Dev. Dyn.

Han, Y., Chen, A., Umansky, K. B., Oonk, K. A., Choi, W. Y., Dickson, A. L., Ou, J., Cigliola, V., Yifa, O., Cao, J., et al. (2019). Vitamin D Stimulates Cardiomyocyte Proliferation and Controls Organ Size and Regeneration in Zebrafish. Dev. Cell.

Hansen, C. G., Bright, N. A., Howard, G. and Nichols, B. J. (2009). SDPR induces membrane curvature and functions in the formation of caveolae. Nat. Cell Biol.

Hansen, C. G., Shvets, E., Howard, G., Riento, K. and Nichols, B. J. (2013). Deletion of cavin genes reveals tissue-specific mechanisms for morphogenesis of endothelial caveolae. Nat. 


\section{Commun.}

Harrison, M. R. M., Bussmann, J., Huang, Y., Zhao, L., Osorio, A., Burns, C. G., Burns, C. E., Sucov, H. M., Siekmann, A. F. and Lien, C. L. (2015). Chemokine-Guided Angiogenesis Directs Coronary Vasculature Formation in Zebrafish. Dev. Cell.

Hasegawa, T., Takeuchi, A., Miyaishi, O., Xiao, H., Mao, J. and Isobe, K. I. (2000). PTRF (polymerase I and transcript-release factor) is tissue-specific and interacts with the BFCOL1 (binding factor of a type-I collagen promoter) zinc-finger transcription factor which binds to the two mouse type-I collagen gene promoters. Biochem. $J$.

Hayashi, K., Banno, H., Kadomatsu, K., Takei, Y., Komori, K. and Muramatsu, T. (2005). Antisense oligodeoxyribonucleotide as to the growth factor midkine suppresses neointima formation induced by balloon injury. Am. J. Physiol. - Hear. Circ. Physiol.

Heallen, T., Morikawa, Y., Leach, J., Tao, G., Willerson, J. T., Johnson, R. L. and Martin, J. F. (2013). Hippo signaling impedes adult heart regeneration. Dev.

Herradon, G., Ezquerra, L., Nguyen, T., Silos-Santiago, I. and Deuel, T. F. (2005). Midkine regulates pleiotrophin organ-specific gene expression: Evidence for transcriptional regulation and functional redundancy within the pleiotrophin/midkine developmental gene family. Biochem. Biophys. Res. Commun.

Hill, M. M., Bastiani, M., Luetterforst, R., Kirkham, M., Kirkham, A., Nixon, S. J., Walser, P., Abankwa, D., Oorschot, V. M. J., Martin, S., et al. (2008). PTRF-Cavin, a Conserved Cytoplasmic Protein Required for Caveola Formation and Function. Cell.

Honda, Y., Shishido, T., Takahashi, T., Watanabe, T., Netsu, S., Kinoshita, D., Narumi, T., Kadowaki, S., Nishiyama, S., Takahashi, H., et al. (2016). Midkine deteriorates cardiac remodeling via epidermal growth factor receptor signaling in chronic kidney disease. Hypertension.

Horiba, M., Kadomatsu, K., Nakamura, E., Muramatsu, H., Ikematsu, S., Sakuma, S., Hayashi, K., Yuzawa, Y., Matsuo, S., Kuzuya, M., et al. (2000). Neointima formation in a restenosis model is suppressed in midkine- deficient mice. J. Clin. Invest.

Horiba, M., Kadomatsu, K., Yasui, K., Lee, J. K., Takenaka, H., Sumida, A., Kamiya, K., Chen, S., Sakuma, S., Muramatsu, T., et al. (2006). Midkine plays a protective role against cardiac ischemia/reperfusion injury through a reduction of apoptotic reaction. Circulation.

Housley, M. P., Njaine, B., Ricciardi, F., Stone, O. A., Hölper, S., Krüger, M., Kostin, S. and Stainier, D. Y. R. (2016). Cavin4b/Murcb Is Required for Skeletal Muscle Development and Function in Zebrafish. PLoS Genet.

Hu, N., Joseph Yost, H. and Clark, E. B. (2001). Cardiac morphology and blood pressure in the adult zebrafish. Anat. Rec.

Huang, W. C., Yang, C. C., Chen, I. H., Liu, Y. M. L., Chang, S. J. and Chuang, Y. J. (2013). Treatment of Glucocorticoids Inhibited Early Immune Responses and Impaired Cardiac 
Repair in Adult Zebrafish. PLoS One.

Hugosson, F., Sjögren, C., Birve, A., Hedlund, L., Eriksson, T. and Palmer, R. H. (2014). The Drosophila midkine/Pleiotrophin homologues Miple1 and Miple2 affect adult lifespan but are dispensable for Alk signaling during embryonic gut formation. PLoS One.

Ikutomo, M., Sakakima, H., Matsuda, F. and Yoshida, Y. (2014). Midkine-deficient mice delayed degeneration and regeneration after skeletal muscle injury. Acta Histochem.

Inoh, K., Muramatsu, H., Ochiai, K., Torii, S. and Muramatsu, T. (2004). Midkine, a heparinbinding cytokine, plays key roles in intraperitoneal adhesions. Biochem. Biophys. Res. Commun.

Ishiguro, H., Horiba, M., Takenaka, H., Sumida, A., Opthof, T., Ishiguro, Y. S., Kadomatsu, K., Murohara, T. and Kodama, I. (2011). A single intracoronary injection of midkine reduces ischemia/reperfusion injury in swine hearts: A novel therapeutic approach for acute coronary syndrome. Front. Physiol.

Itou, J., Oishi, I., Kawakami, H., Glass, T. J., Richter, J., Johnson, A., Lund, T. C. and Kawakami, Y. (2012). Migration of cardiomyocytes is essential for heart regeneration in zebrafish. Dev.

Jasmin, J. F., Rengo, G., Lymperopoulos, A., Gupta, R., Eaton, G. J., Quann, K., Gonzales, D. M., Mercier, I., Koch, W. J. and Lisanti, M. P. (2011). Caveolin-1 deficiency exacerbates cardiac dysfunction and reduces survival in mice with myocardial infarction. Am. J. Physiol. - Hear. Circ. Physiol.

Jiang, L., Romero-Carvajal, A., Haug, J. S., Seidel, C. W. and Piotrowski, T. (2014). Geneexpression analysis of hair cell regeneration in the zebrafish lateral line. Proc. Natl. Acad. Sci. U.S. A.

Johnson, S. L. and Weston, J. A. (1995). Temperature-sensitive mutations that cause stagespecific defects in zebrafish fin regeneration. Genetics.

Jopling, C., Sleep, E., Raya, M., Martí, M., Raya, A. and Belmonte, J. C. I. (2010). Zebrafish heart regeneration occurs by cardiomyocyte dedifferentiation and proliferation. Nature.

Jopling, C., Suñé, G., Faucherre, A., Fabregat, C. and Izpisua Belmonte, J. C. (2012). Hypoxia induces myocardial regeneration in zebrafish. Circulation.

Kadomatsu, K., Tomomura, M. and Muramatsu, T. (1988). cDNA cloning and sequencing of a new gene intensely expressed in early differentiation stages of embryonal carcinoma cells and in mid-gestation period of mouse embryogenesis. Biochem. Biophys. Res. Commun.

Kadomatsu, K., Hung, R. P., Suganuma, T., Murata, F. and Muramatsu, T. (1990). A retinoic acid responsive gene MK found in the teratocarcinoma system is expressed in spatially and temporally controlled manner during mouse embryogenesis. J. Cell Biol.

Kang, J., Hu, J., Karra, R., Dickson, A. L., Tornini, V. A., Nachtrab, G., Gemberling, M., Goldman, J. A., Black, B. L. and Poss, K. D. (2016). Modulation of tissue repair by 
regeneration enhancer elements. Nature.

Karra, R., Knecht, A. K., Kikuchi, K. and Poss, K. D. (2015). Myocardial NF-kB activation is essential for zebrafish heart regeneration. Proc. Natl. Acad. Sci. U. S. A.

Kendall, R. T. and Feghali-Bostwick, C. A. (2014). Fibroblasts in fibrosis: Novel roles and mediators. Front. Pharmacol.

Khan, A., Fornes, O., Stigliani, A., Gheorghe, M., Castro-Mondragon, J. A., Van Der Lee, R., Bessy, A., Chèneby, J., Kulkarni, S. R., Tan, G., et al. (2018). JASPAR 2018: Update of the open-access database of transcription factor binding profiles and its web framework. Nucleic Acids Res.

Kikuchi, K., Holdway, J. E., Werdich, A. A., Anderson, R. M., Fang, Y., Egnaczyk, G. F., Evans, T., MacRae, C. A., Stainier, D. Y. R. and Poss, K. D. (2010). Primary contribution to zebrafish heart regeneration by gata4+ cardiomyocytes. Nature.

Kikuchi, K., Gupta, V., Wang, J., Holdway, J. E., Wills, A. A., Fang, Y. and Poss, K. D. (2011a). tcf21+ epicardial cells adopt non-myocardial fates during zebrafish heart development and regeneration. Development.

Kikuchi, K., Holdway, J. E., Major, R. J., Blum, N., Dahn, R. D., Begemann, G. and Poss, K. D. (2011b). Retinoic Acid Production by Endocardium and Epicardium Is an Injury Response Essential for Zebrafish Heart Regeneration. Dev. Cell.

Kikuchi, K., Holdway, J. E., Major, R. J., Blum, N., Dahn, R. D., Begemann, G. and Poss, K. D. (2011c). Retinoic Acid Production by Endocardium and Epicardium Is an Injury Response Essential for Zebrafish Heart Regeneration. Dev. Cell.

Kim, C. A., Delépine, M., Boutet, E., El Mourabit, H., Le Lay, S., Meier, M., Nemani, M., Bridel, E., Leite, C. C., Bertola, D. R., et al. (2008a). Association of a homozygous nonsense caveolin-1 mutation with berardinelli-seip congenital lipodystrophy. J. Clin. Endocrinol. Metab.

Kim, H., Shin, J., Kim, S., Poling, J., Park, H. C. and Appel, B. (2008b). Notch-regulated oligodendrocyte specification from radial glia in the spinal cord of zebrafish embryos. Dev. Dyn.

Kim, J., Wu, Q., Zhang, Y., Wiens, K. M., Huang, Y., Rubin, N., Shimada, H., Handin, R. I., Chao, M. Y., Tuan, T. L., et al. (2010). PDGF signaling is required for epicardial function and blood vessel formation in regenerating zebrafish hearts. Proc. Natl. Acad. Sci. U. S. A.

Kimmel, C. B., Ballard, W. W., Kimmel, S. R., Ullmann, B. and Schilling, T. F. (1995). Stages of embryonic development of the zebrafish. Dev. Dyn.

King, B. L. and Yin, V. P. (2016). A conserved microRNA regulatory circuit is differentially controlled during limb/appendage regeneration. PLoS One.

Kirkham, M., Nixon, S. J., Howes, M. T., Abi-Rached, L., Wakeham, D. E., Hanzal-Bayer, M., Ferguson, C., Hill, M. M., Fernandez-Rojo, M., Brown, D. A., et al. (2008). 
Evolutionary analysis and molecular dissection of caveola biogenesis. J. Cell Sci.

Koleske, A. J., Baltimore, D. and Lisanti, M. P. (1995). Reduction of caveolin and caveolae in oncogenically transformed cells. Proc. Natl. Acad. Sci. U. S. A.

Kroehne, V., Freudenreich, D., Hans, S., Kaslin, J. and Brand, M. (2011). Regeneration of the adult zebrafish brain from neurogenic radial glia-type progenitors. Development.

Kurzchalia, T. V., Dupree, P., Parton, R. G., Kellner, R., Virta, H., Lehnert, M. and Simons, K. (1992). VIP21, a 21-kD membrane protein is an integral component of trans-Golginetwork-derived transport vesicles. J. Cell Biol.

Lackner, Weber, B., Baur, M., Haffner-Luntzer, M., Eiseler, T., Fois, G., Gebhard, F., Relja, B., Marzi, I., Pfeifer, R., Halvachizadeh, S., et al. (2019). Midkine Is Elevated After Multiple Trauma and Acts Directly on Human Cardiomyocytes by Altering Their Functionality and Metabolism. Front. Immunol.

Lafontant, P. J., Behzad, A. R., Brown, E., Landry, P., Hu, N. and Burns, A. R. (2013). Cardiac Myocyte Diversity and a Fibroblast Network in the Junctional Region of the Zebrafish Heart Revealed by Transmission and Serial Block-Face Scanning Electron Microscopy. PLoS One.

Lai, S. L., Marín-Juez, R., Moura, P. L., Kuenne, C., Lai, J. K. H., Tsedeke, A. T., Guenther, S., Looso, M. and Stainier, D. Y. R. (2017). Reciprocal analyses in zebrafish and medaka reveal that harnessing the immune response promotes cardiac regeneration. Elife.

Lawson, N. D. and Weinstein, B. M. (2002). In vivo imaging of embryonic vascular development using transgenic zebrafish. Dev. Biol.

Lepilina, A., Coon, A. N., Kikuchi, K., Holdway, J. E., Roberts, R. W., Burns, C. G. and Poss, K. D. (2006). A Dynamic Epicardial Injury Response Supports Progenitor Cell Activity during Zebrafish Heart Regeneration. Cell.

Li, Y. S., Milner, P. G., Chauhan, A. K., Watson, M. A., Hoffman, R. M., Kodner, C. M., Milbrandt, J. and Deuel, T. F. (1990). Cloning and expression of a developmentally regulated protein that induces mitogenic and neurite outgrowth activity. Science (80-. ).

Lim, Y. W., Lo, H. P., Ferguson, C., Martel, N., Giacomotto, J., Gomez, G. A., Yap, A. S., Hall, T. E. and Parton, R. G. (2017). Caveolae Protect Notochord Cells against Catastrophic Mechanical Failure during Development. Curr. Biol.

Lin, Z., Von Gise, A., Zhou, P., Gu, F., Ma, Q., Jiang, J., Yau, A. L., Buck, J. N., Gouin, K. A., Van Gorp, P. R. R., et al. (2014). Cardiac-specific YAP activation improves cardiac function and survival in an experimental murine MI model. Circ. Res.

Liu, L., Brown, D., McKee, M., LeBrasseur, N. K., Yang, D., Albrecht, K. H., Ravid, K. and Pilch, P. F. (2008). Deletion of Cavin/PTRF Causes Global Loss of Caveolae, Dyslipidemia, and Glucose Intolerance. Cell Metab.

Lo, H. P., Nixon, S. J., Hall, T. E., Cowling, B. S., Ferguson, C., Morgan, G. P., Schieber, N. L., Fernandez-Rojo, M. A., Bastiani, M., Floetenmeyer, M., et al. (2015). The caveolin- 
Cavin system plays a conserved and critical role in mechanoprotection of skeletal muscle. $J$. Cell Biol.

Lowe, V., Wisniewski, L., Sayers, J., Evans, I., Frankel, P., Mercader-Huber, N., Zachary, I. C. and Pellet-Many, C. (2019). Neuropilin 1 mediates epicardial activation and revascularization in the regenerating zebrafish heart. Development.

Luo, J., Uribe, R. A., Hayton, S., Calinescu, A. A., Gross, J. M. and Hitchcock, P. F. (2012). Midkine-A functions upstream of Id2a to regulate cell cycle kinetics in the developing vertebrate retina. Neural Dev.

Marín-Juez, R., Marass, M., Gauvrit, S., Rossi, A., Lai, S.-L., Materna, S. C., Black, B. L. and Stainier, D. Y. R. (2016). Fast revascularization of the injured area is essential to support zebrafish heart regeneration. Proc. Natl. Acad. Sci.

Marro, J., Pfefferli, C., De Charles, A. S. P., Bise, T. and Jaźwiñska, A. (2016). Collagen XII contributes to epicardial and connective tissues in the zebrafish heart during ontogenesis and regeneration. PLoS One.

März, M., Chapouton, P., Diotel, N., Vaillant, C., Hesl, B., Takamiya, M., Lam, C. S., Kah, O., Bally-Cuif, L. and Strähle, U. (2010). Heterogeneity in progenitor cell subtypes in the ventricular zone of the zebrafish adult telencephalon. Glia.

Mathur, A. B., Collinsworth, A. M., Reichert, W. M., Kraus, W. E. and Truskey, G. A. (2001). Endothelial, cardiac muscle and skeletal muscle exhibit different viscous and elastic properties as determined by atomic force microscopy. J. Biomech.

Matsubara, S., Take, M., Pedraza, C. and Muramatsu, T. (1994). Mapping and characterization of a retinoic acid-responsive enhancer of midkine, a novel heparin-binding growth/differentiation factor with neurotrophic activity. J. Biochem.

McCurley, A. T. and Callard, G. V. (2008). Characterization of housekeeping genes in zebrafish: Male-female differences and effects of tissue type, developmental stage and chemical treatment. BMC Mol. Biol.

McMahon, K. A., Zajicek, H., Li, W. P., Peyton, M. J., Minna, J. D., Hernandez, V. J., LubyPhelps, K. and Anderson, R. G. W. (2009). SRBC/cavin-3 is a caveolin adapter protein that regulates caveolae function. $E M B O J$.

Michikawa, M., Kikuchi, S., Muramatsu, H., Muramatsu, T. and Kim, S. U. (1993). Retinoic acid responsive gene product, midkine, has neurotrophic functions for mouse spinal cord and dorsal root ganglion neurons in culture. J. Neurosci. Res.

Misa, K., Tanino, Y., Wang, X., Nikaido, T., Kikuchi, M., Sato, Y., Togawa, R., Tanino, M., Tanaka, S., Kadomatsu, K., et al. (2017). Involvement of midkine in the development of pulmonary fibrosis. Physiol. Rep.

Missinato, M. A., Saydmohammed, M., Zuppo, D. A., Rao, K. S., Opie, G. W., Kühn, B. and Tsang, M. (2018). Dusp6 attenuates Ras/MAPK signaling to limit zebrafish heart 
regeneration. Dev.

Mitsiadis, T. A., Salmivirta, M., Muramatsu, T., Muramatsu, H., Rauvala, H., Lehtonen, E., Jalkanen, M. and Thesleff, I. (1995). Expression of the heparin-binding cytokines, midkine (MK) and HB-GAM (pleiotrophin) is associated with epithelial-mesenchymal interactions during fetal development and organogenesis. Development.

Miyasato, S. K., Loeffler, J., Shohet, R., Zhang, J., Lindsey, M. and Le Saux, C. J. (2011). Caveolin-1 modulates TGF- $\beta 1$ signaling in cardiac remodeling. Matrix Biol.

Monier, S., Dietzen, D. J., Hastings, W. R., Lublin, D. M. and Kurzchalia, T. V. (1996). Oligomerization of VIP21-caveolin in vitro is stabilized by long chain fatty acylation or cholesterol. FEBS Lett.

Mora, R., Bonilha, V. L., Marmorstein, A., Scherer, P. E., Brown, D., Lisanti, M. P. and Rodriguez-Boulan, E. (1999). Caveolin-2 localizes to the Golgi complex but redistributes to plasma membrane, caveolae, and rafts when co-expressed with caveolin-1. J. Biol. Chem.

Moreno-Vicente, R., Pavón, D. M., Martín-Padura, I., Català-Montoro, M., Díez-Sánchez, A., Quílez-Álvarez, A., López, J. A., Sánchez-Álvarez, M., Vázquez, J., Strippoli, R., et al. (2018). Caveolin-1 Modulates Mechanotransduction Responses to Substrate Stiffness through Actin-Dependent Control of YAP. Cell Rep.

Moreno, R. L., Williams, K., Jones, K. L. and Ribera, A. B. (2018). Investigation of Islet2a function in zebrafish embryos: Mutants and morphants differ in morphologic phenotypes and gene expression. PLoS One.

Münch, J., Grivas, D., González-Rajal, Á., Torregrosa-Carrión, R. and de la Pompa, J. L. (2017). Notch signalling restricts inflammation and serpinel expression in the dynamic endocardium of the regenerating zebrafish heart . Development.

Muramatsu, H., Shirahama, H., Yonezawa, S., Maruta, H. and Muramatsu, T. (1993). Midkine, A retinoic acid-inducible growth/differentiation factor: Immunochemical evidence for the function and distribution. Dev. Biol.

Muramatsul, H., Yokoi, K., Chen, L., Ichihara-Tanaka, K., Kimura, T. and Muramatsu, T. (2011). Midkine as a factor to counteract the deposition of amyloid -peptide plaques: In vitro analysis and examination in knockout mice. Int. Arch. Med.

Murata, T., Lin, M. I., Huang, Y., Yu, J., Bauer, P. M., Giordano, F. J. and Sessa, W. C. (2007). Reexpression of caveolin-1 in endothelium rescues the vascular, cardiac, and pulmonary defects in global caveolin-1 knockout mice. J. Exp. Med.

Nakagawara, A., Takao, S., Shimazu, H. and Muramatsu, T. (1993). A New Family of Heparinbinding Growth/Differentiation Factors: Increased Midkine Expression in Wilms9 Tumor and Other Human Carcinomas1. Cancer Res.

Nakamura, E., Kadomatsu, K., Yuasa, S., Muramatsu, H., Mamiya, T., Nabeshima, T., Fan, Q. W., Ishiguro, K., Igakura, T., Matsubara, S., et al. (1998). Disruption of the midkine 
gene (Mdk) resulted in altered expression of a calcium binding protein in the hippocampus of infant mice and their abnormal behaviour. Genes to Cells.

Nechiporuk, A. and Keating, M. T. (2002). A proliferation gradient between proximal and msxbexpressing distal blastema directs zebrafish fin regeneration. Development.

Netsu, S., Shishido, T., Kitahara, T., Honda, Y., Funayama, A., Narumi, T., Kadowaki, S., Takahashi, H., Miyamoto, T., Arimoto, T., et al. (2014). Midkine exacerbates pressure overload-induced cardiac remodeling. Biochem. Biophys. Res. Commun.

Nivón, L. G., Moretti, R. and Baker, D. (2013). A Pareto-Optimal Refinement Method for Protein Design Scaffolds. PLoS One.

Nixon, S. J., Wegner, J., Ferguson, C., Méry, P. F., Hancock, J. F., Currie, P. D., Key, B., Westerfield, M. and Parton, R. G. (2005). Zebrafish as a model for caveolin-associated muscle disease; caveolin-3 is required for myofibril organization and muscle cell patterning. Hum. Mol. Genet.

Nixon, S. J., Carter, A., Wegner, J., Ferguson, C., Floetenmeyer, M., Riches, J., Key, B., Westerfield, M. and Parton, R. G. (2007). Caveolin-1 is required for lateral line neuromast and notochord development. J. Cell Sci.

Obama, H., Biro, S., Tashiro, T., Tsutsui, J. I., Ozawa, M., Yoshida, H., Tanaka, H. and Muramatsu, T. (1998). Myocardial infarction induces expression of midkine, a heparinbinding growth factor with reparative activity. Anticancer Res.

Obermeier, B., Daneman, R. and Ransohoff, R. M. (2013). Development, maintenance and disruption of the blood-brain barrier. Nat. Med.

Ogata, T., Naito, D., Nakanishi, N., Hayashi, Y. K., Taniguchi, T., Miyagawa, K., Hamaoka, T., Maruyama, N., Matoba, S., Ikeda, K., et al. (2014). MURC/Cavin-4 facilitates recruitment of ERK to caveolae and concentric cardiac hypertrophy induced by $\alpha 1$-adrenergic receptors. Proc. Natl. Acad. Sci. U. S. A.

Okuda, Y., Yoda, H., Uchikawa, M., Furutani-Seiki, M., Takeda, H., Kondoh, H. and Kamachi, Y. (2006). Comparative genomic and expression analysis of group B1 sox genes in zebrafish indicates their diversification during vertebrate evolution. Dev. Dyn.

Palade, G. E. (1953). An electron microscope study of the mitochondrial structure. J. Histochem. Cytochem.

Palmer, R. M. J., Ferrige, A. G. and Moncada, S. (1987). Nitric oxide release accounts for the biological activity of endothelium-derived relaxing factor. Nature.

Park, D. S., Cohen, A. W., Frank, P. G., Razani, B., Lee, H., Williams, T. M., Chandra, M., Shirani, J., De Souza, A. P., Tang, B., et al. (2003). Caveolin-1 Null (-/-) Mice Show Dramatic Reductions in Life Span. Biochemistry.

Park, C., Kim, T. M. and Malik, A. B. (2013). Transcriptional regulation of endothelial cell and vascular development. Circ. Res. 
Parton, R. G., Hanzal-Bayer, M. and Hancock, J. F. (2006). Biogenesis of caveolae: A structural model for caveolin-induced domain formation. J. Cell Sci.

Patel, H. H., Tsutsumi, Y. M., Head, B. P., Niesman, I. R., Jennings, M., Horikawa, Y., Huang, D., Moreno, A. L., Patel, P. M., Insel, P. A., et al. (2007). Mechanisms of cardiac protection from ischemia/reperfusion injury: A role for caveolae and caveolin-1. FASEB $J$.

Peng, F., Wu, D., Ingram, A. J., Zhang, B., Gao, B. and Krepinsky, J. C. (2007). RhoA activation in mesangial cells by mechanical strain depends on caveolae and caveolin-1 interaction. J. Am. Soc. Nephrol.

Peralta, M., Steed, E., Harlepp, S., González-Rosa, J. M., Monduc, F., Ariza-Cosano, A., Cortés, A., Rayón, T., Gómez-Skarmeta, J. L., Zapata, A., et al. (2013). Heartbeat-driven pericardiac fluid forces contribute to epicardium morphogenesis. Curr. Biol.

Perner, B., Englert, C. and Bollig, F. (2007). The Wilms tumor genes wt1a and wt1b control different steps during formation of the zebrafish pronephros. Dev. Biol.

Pestel, J., Ramadass, R., Gauvrit, S., Helker, C., Herzog, W. and Stainier, D. Y. R. (2016). Real-time 3D visualization of cellular rearrangements during cardiac valve formation. Dev.

Pfefferli, C. and Jaźwińska, A. (2017). The careg element reveals a common regulation of regeneration in the zebrafish myocardium and fin. Nat. Commun.

Pistocchi, A., Feijáo, C. G., Cabrera, P., Villablanca, E. J., Allende, M. L. and Cotelli, F. (2009). The zebrafish prospero homolog prox1 is required for mechanosensory hair cell differentiation and functionality in the lateral line. BMC Dev. Biol.

Porrello, E. R., Mahmoud, A. I., Simpson, E., Hill, J. A., Richardson, J. A., Olson, E. N. and Sadek, H. A. (2011). Transient regenerative potential of the neonatal mouse heart. Science (80-. ).

Poss, K. D., Wilson, L. G. and Keating, M. T. (2002). Heart regeneration in zebrafish. Science (80-.).

Quaife-Ryan, G. A., Sim, C. B., Ziemann, M., Kaspi, A., Rafehi, H., Ramialison, M., El-Osta, A., Hudson, J. E. and Porrello, E. R. (2017). Multicellular transcriptional analysis of mammalian heart regeneration. Circulation.

Quillien, A., Abdalla, M., Yu, J., Ou, J., Zhu, L. J. and Lawson, N. D. (2017). Robust Identification of Developmentally Active Endothelial Enhancers in Zebrafish Using FANSAssisted ATAC-Seq. Cell Rep.

Rabinowitz, J. S., Robitaille, A. M., Wang, Y., Ray, C. A., Thummel, R., Gu, H., Djukovic, D., Raftery, D., Berndt, J. D. and Moon, R. T. (2017). Transcriptomic, proteomic, and metabolomic landscape of positional memory in the caudal fin of zebrafish. Proc. Natl. Acad. Sci. U. S. A.

Raible, D. W. and Kruse, G. J. (2000). Organization of the lateral line system in embryonic zebrafish. J. Comp. Neurol. 
Razani, B., Engelman, J. A., Wang, X. B., Schubert, W., Zhang, X. L., Marks, C. B., Macalusol, F., Russell, R. G., Li, M., Pestell, R. G., et al. (2001a). Caveolin-1 Null Mice Are Viable but Show Evidence of Hyperproliferative and Vascular Abnormalities. J. Biol. Chem.

Razani, B., Zhang, X. L., Bitzer, M., Von Gersdorff, G., Böttinger, E. P. and Lisanti, M. P. (2001b). Caveolin-1 Regulates Transforming Growth Factor (TGF)- $\beta /$ SMAD Signaling through an Interaction with the TGF- $\beta$ Type I Receptor. J. Biol. Chem.

Razani, B., Wang, X. B., Engelman, J. A., Battista, M., Lagaud, G., Zhang, X. L., Kneitz, B., Hou, H., Christ, G. J., Edelmann, W., et al. (2002). Caveolin-2-Deficient Mice Show Evidence of Severe Pulmonary Dysfunction without Disruption of Caveolae. Mol. Cell. Biol.

Reischauer, S., Arnaout, R., Ramadass, R. and Stainier, D. Y. R. (2014). Actin binding GFP allows 4D in vivo imaging of myofilament dynamics in the zebrafish heart and the identification of ErBB2 signaling as a remodeling factor of myofibril architecture. Circ. Res.

Ren, X. (2004). Conformational Defects Slow Golgi Exit, Block Oligomerization, and Reduce Raft Affinity of Caveolin-1 Mutant Proteins. Mol. Biol. Cell.

Reynolds, E. S. (1963). THE USE OF LEAD CITRATE AT HIGH pH AS AN ELECTRONOPAQUE STAIN IN ELECTRON MICROSCOPY. J. Cell Biol.

Reynolds, P. R., Mucenski, M. L., Le Cras, T. D., Nichols, W. C. and Whitsett, J. A. (2004). Midkine is regulated by hypoxia and causes pulmonary vascular remodeling. J. Biol. Chem.

Robenek, H., Weissen-Plenz, G. and Severs, N. J. (2008a). Freeze-fracture replica immunolabelling reveals caveolin-1 in the human cardiomyocyte plasma membrane. J Cell Mol Med.

Robenek, H., Weissen-Plenz, G. and Severs, N. J. (2008b). Freeze-fracture replica immunolabelling reveals caveolin-1 in the human cardiomyocyte plasma membrane. J. Cell. Mol. Med.

Rohr, S., Otten, C. and Abdelilah-Seyfried, S. (2008). Asymmetric involution of the myocardial field drives heart tube formation in zebrafish. Circ. Res.

Rojas, D. A., Perez-Munizaga, D. A., Centanin, L., Antonelli, M., Wappner, P., Allende, M. L. and Reyes, A. E. (2007). Cloning of hif- $1 \alpha$ and hif- $2 \alpha$ and mRNA expression pattern during development in zebrafish. Gene Expr. Patterns.

Rothbart, S. B. and Strahl, B. D. (2014). Interpreting the language of histone and DNA modifications. Biochim. Biophys. Acta - Gene Regul. Mech.

Rothberg, K. G., Heuser, J. E., Donzell, W. C., Ying, Y. S., Glenney, J. R. and Anderson, R. G. W. (1992). Caveolin, a protein component of caveolae membrane coats. Cell.

Sánchez-Iranzo, H., Galardi-Castilla, M., Sanz-Morejón, A., González-Rosa, J. M., Costa, R., Ernst, A., Sainz de Aja, J., Langa, X. and Mercader, N. (2018a). Transient fibrosis resolves via fibroblast inactivation in the regenerating zebrafish heart. Proc. Natl. Acad. Sci. 
Sánchez-Iranzo, H., Galardi-Castilla, M., Minguillón, C., Sanz-Morejón, A., González-Rosa, J. M., Felker, A., Ernst, A., Guzmán-Martínez, G., Mosimann, C. and Mercader, N. (2018b). Tbx5a lineage tracing shows cardiomyocyte plasticity during zebrafish heart regeneration. Nat. Commun.

Sanz-Morejón, A., García-Redondo, A. B., Reuter, H., Marques, I. J., Bates, T., GalardiCastilla, M., Große, A., Manig, S., Langa, X., Ernst, A., et al. (2019). Wilms Tumor 1b Expression Defines a Pro-regenerative Macrophage Subtype and Is Required for Organ Regeneration in the Zebrafish. Cell Rep.

Sato, W., Kadomatsu, K., Yuzawa, Y., Muramatsu, H., Hotta, N., Matsuo, S. and Muramatsu, T. (2001). Midkine Is Involved in Neutrophil Infiltration into the Tubulointerstitium in Ischemic Renal Injury. J. Immunol.

Schäfer, M., Rembold, M., Wittbrodt, J., Schartl, M. and Winkler, C. (2005). Medial floor plate formation in zebrafish consists of two phases and requires trunk-derived Midkine-a. Genes Dev.

Scherer, P. E., Okamoto, T., Chun, M., Nishimoto, I., Lodish, H. F. and Lisanti, M. P. (1996). Identification, sequence, and expression of caveolin-2 defines a caveolin gene family. Proc. Natl. Acad. Sci. U. S. A.

Scherer, P. E., Lewis, R. Y., Volonté, D., Engelman, J. A., Galbiati, F., Couet, J., Kohtz, D. S., Van Donselaar, E., Peters, P. and Lisanti, M. P. (1997). Cell-type and tissue-specific expression of caveolin-2. Caveolins 1 and 2 co-localize and form a stable hetero-oligomeric complex in vivo. J. Biol. Chem.

Schlegel, A. and Lisanti, M. P. (2000). A Molecular Dissection of Caveolin-1 Membrane Attachment and Oligomerization. J. Biol. Chem.

Schlegel, A., Schwab, R. B., Scherer, P. E. and Lisanti, M. P. (1999). A role for the caveolin scaffolding domain in mediating the membrane attachment of caveolin-1. The caveolin scaffolding domain is both necessary and sufficient for membrane binding in vitro. J. Biol. Chem.

Schmidt, R., Strähle, U. and Scholpp, S. (2013). Neurogenesis in zebrafish - from embryo to adult. Neural Dev.

Schnabel, K., Wu, C. C., Kurth, T. and Weidinger, G. (2011). Regeneration of cryoinjury induced necrotic heart lesions in zebrafish is associated with epicardial activation and cardiomyocyte proliferation. PLoS One.

Schrödinger, L. (2015). The PyMOL molecular graphics system, version 1.8. https://www.pymol.org/citing.

Sedmera, D., Pexieder, T., Vuillemin, M., Thompson, R. P. and Anderson, R. H. (2000). Developmental patterning of the myocardium. Anat. Rec.

Semenza, G. L. (2000). Hif-1 and human disease: One highly involved factor. Genes Dev. 
Shisan Xu (2019). Prolonged neutrophil retention in the wound impairs zebrafish heart regeneration after cryoinjury. Fish Shellfish Immunol.

Shivshankar, P., Halade, G. V., Calhoun, C., Escobar, G. P., Mehr, A. J., Jimenez, F., Martinez, C., Bhatnagar, H., Mjaatvedt, C. H., Lindsey, M. L., et al. (2014). Caveolin-1 deletion exacerbates cardiac interstitial fibrosis by promoting M2 macrophage activation in mice after myocardial infarction. Curr. Ther. Res. - Clin. Exp.

Sinha, B., Köster, D., Ruez, R., Gonnord, P., Bastiani, M., Abankwa, D., Stan, R. V, ButlerBrowne, G., Vedie, B., Johannes, L., et al. (2011). Cells respond to mechanical stress by rapid disassembly of caveolae. Cell.

Song, K. S., Tang, Z., Li, S. and Lisanti, M. P. (1997). Mutational analysis of the properties of caveolin-1. A novel role for the C-terminal domain in mediating homo-typic caveolincaveolin interactions. J. Biol. Chem.

Souders, C. A., Bowers, S. L. K. and Baudino, T. A. (2009). Cardiac fibroblast: The renaissance cell. Circ. Res.

Sowa, G., Pypaert, M. and Sessa, W. C. (2001). Distinction between signaling mechanisms in lipid rafts vs. caveolae. Proc. Natl. Acad. Sci.

St. John Sutton, M. G. and Sharpe, N. (2000). Left ventricular remodeling after myocardial infarction: Pathophysiology and therapy. Circulation.

Steiner, A. B., Kim, T., Cabot, V. and Hudspeth, A. J. (2014). Dynamic gene expression by putative hair-cell progenitors during regeneration in the zebrafish lateral line. Proc. Natl. Acad. Sci. U. S. A.

Stoeber, M., Schellenberger, P., Siebert, C. A., Leyrat, C., Grünewald, K. and Helenius, A. (2016). Model for the architecture of caveolae based on a flexible, net-like assembly of Cavin1 and Caveolin discs. Proc. Natl. Acad. Sci. U. S. A.

Sumi, Y., Muramatsu, H., Takei, Y., Hata, K. I., Ueda, M. and Muramatsu, T. (2002). Midkine, a heparin-binding growth factor, promotes growth and glycosaminoglycan synthesis of endothelial cells through its action on smooth muscle cells in an artificial blood vessel model. J. Cell Sci.

Sumida, A., Horiba, M., Ishiguro, H., Takenaka, H., Ueda, N., Ooboshi, H., Opthof, T., Kadomatsu, K. and Kodama, I. (2010). Midkine gene transfer after myocardial infarction in rats prevents remodelling and ameliorates cardiac dysfunction. Cardiovasc. Res.

Susaki, E. A., Tainaka, K., Perrin, D., Kishino, F., Tawara, T., Watanabe, T. M., Yokoyama, C., Onoe, H., Eguchi, M., Yamaguchi, S., et al. (2014). Whole-brain imaging with singlecell resolution using chemical cocktails and computational analysis. Cell.

Takada, T., Toriyama, K., Muramatsu, H., Song, X. J., Torii, S. and Muramatsu, T. (1997). Midkine, a retinoic acid-inducible heparin-binding cytokine in inflammatory responses: Chemotactic activity to neutrophils and association with inflammatory synovitis. J. Biochem. 
Takenaka, H., Horiba, M., Ishiguro, H., Sumida, A., Hojo, M., Usui, A., Akita, T., Sakuma, S., Ueda, Y., Kodama, I., et al. (2009). Midkine prevents ventricular remodeling and improves long-term survival after myocardial infarction. Am. J. Physiol. - Hear. Circ. Physiol.

Tallafuss, A., Hale, L. A., Yan, Y. L., Dudley, L., Eisen, J. S. and Postlethwait, J. H. (2006). Characterization of retinoid-X receptor genes rxra, rxrba, rxrbb and rxrg during zebrafish development. Gene Expr. Patterns.

Tang, Z., Scherer, P. E., Okamoto, T., Song, K., Chu, C., Kohtz, D. S., Nishimoto, I., Lodish, H. F. and Lisanti, M. P. (1996). Molecular cloning of caveolin-3, a novel member of the caveolin gene family expressed predominantly in muscle. J. Biol. Chem.

Taniguchi, T., Maruyama, N., Ogata, T., Kasahara, T., Nakanishi, N., Miyagawa, K., Naito, D., Hamaoka, T., Nishi, M., Matoba, S., et al. (2016). PTRF/Cavin-1 deficiency causes cardiac dysfunction accompanied by cardiomyocyte hypertrophy and cardiac fibrosis. PLoS One.

Thisse, C., and Thisse, B. (2004). Fast Release Clones: A High Throughput Expression Analysis. ZFIN Direct Data Submiss.

Thisse, B. (2001). Expression of the zebrafish genome during embryogenesis. ZFIN Direct Data Submiss.

Thompson, M. A., Ransom, D. G., Pratt, S. J., MacLennan, H., Kieran, M. W., Detrich, H. W., Vail, B., Huber, T. L., Paw, B., Brownlie, A. J., et al. (1998). The cloche and spadetail genes differentially affect hematopoiesis and vasculogenesis. Dev. Biol.

Trevarrow, B. (2008). Antibody Descriptions. ZFIN Direct Data Submission. http://zfin.org.

Van Leeuwen, L. M., Evans, R. J., Jim, K. K., Verboom, T., Fang, X., Bojarczuk, A., Malicki, J., Johnston, S. A. and van der Sar, A. M. (2018). A transgenic zebrafish model for the in vivo study of the blood and choroid plexus brain barriers using claudin 5. Biol. Open.

van Wijk, B., Gunst, Q. D., Moorman, A. F. M. and van den Hoff, M. J. B. (2012). Cardiac Regeneration from Activated Epicardium. PLoS One.

Vargas-González, A., Prado-Zayago, E., León-Olea, M., Guarner-Lans, V. and CanoMartínez, A. (2005). Myocardial regeneration in Ambystoma mexicanum after surgical injury. Arch. Cardiol. Mex.

Vihtelic, T. S. and Hyde, D. R. (2000). Light-induced rod and cone cell death and regeneration in the adult albino zebrafish (Danio rerio) retina. J. Neurobiol.

Vivien, C. J., Pichol-Thievend, C., Sim, C. B., Smith, J. B., Bower, N. I., Hogan, B. M., Hudson, J. E., Francois, M. and Porrello, E. R. (2019). Vegfc/d-dependent regulation of the lymphatic vasculature during cardiac regeneration is influenced by injury context. npj Regen. Med.

Von Gise, A., Lin, Z., Schlegelmilch, K., Honor, L. B., Pan, G. M., Buck, J. N., Ma, Q., Ishiwata, T., Zhou, B., Camargo, F. D., et al. (2012). YAP1, the nuclear target of Hippo 
signaling, stimulates heart growth through cardiomyocyte proliferation but not hypertrophy. Proc. Natl. Acad. Sci. U. S. A.

Wang, H., Cao, R., Xia, L., Erdjument-Bromage, H., Borchers, C., Tempst, P. and Zhang, Y. (2001). Purification and functional characterization of a histone H3-lysine 4-specific methyltransferase. Mol. Cell.

Wang, J., Panáková, D., Kikuchi, K., Holdway, J. E., Gemberling, M., Burris, J. S., Singh, S. P., Dickson, A. L., Lin, Y.-F., Sabeh, M. K., et al. (2011). The regenerative capacity of zebrafish reverses cardiac failure caused by genetic cardiomyocyte depletion. Development.

Wang, J., Karra, R., Dickson, A. L. and Poss, K. D. (2013). Fibronectin is deposited by injuryactivated epicardial cells and is necessary for zebrafish heart regeneration. Dev. Biol.

Wang, J., Cao, J., Dickson, A. L. and Poss, K. D. (2015). Epicardial regeneration is guided by cardiac outflow tract and Hedgehog signalling. Nature.

Way, M. and Parton, R. G. (1995). M-caveolin, a muscle-specific caveolin-related protein. FEBS Lett.

Webb, K. J., Coolen, M., Gloeckner, C. J., Stigloher, C., Bahn, B., Topp, S., Ueffing, M. and Bally-Cuif, L. (2011). The enhancer of split transcription factor Her8a is a novel dimerisation partner for Her3 that controls anterior hindbrain neurogenesis in zebrafish. BMC Dev. Biol.

Weckbach, L. T., Groesser, L., Borgolte, J., Pagel, J. I., Pogoda, F., Schymeinsky, J., MüllerHöcker, J., Shakibaei, M., Muramatsu, T., Deindl, E., et al. (2012). Midkine acts as proangiogenic cytokine in hypoxia-induced angiogenesis. Am. J. Physiol. - Hear. Circ. Physiol.

Weckbach, L. T., Gola, A., Winkelmann, M., Jakob, S. M., Groesser, L., Borgolte, J., Pogoda, F., Pick, R., Pruenster, M., Müller-Höcker, J., et al. (2014). The cytokine midkine supports neutrophil trafficking during acute inflammation by promoting adhesion via $\beta 2$ integrins (CD11/CD18). Blood.

Weckbach, L. T., Grabmaier, U., Uhl, A., Gess, S., Boehm, F., Zehrer, A., Pick, R., Salvermoser, M., Czermak, T., Pircher, J., et al. (2019). Midkine drives cardiac inflammation by promoting neutrophil trafficking and NETosis in myocarditis. J. Exp. Med.

Williams, J. A. and Holder, N. (2000). Cell turnover in neuromasts of zebrafish larvae. Hear. Res.

Winkler, C., Schäfer, M., Duschl, J., Schartl, M. and Volff, J. N. (2003). Functional divergence of two zebrafish midkine growth factors following fish-specific gene duplication. Genome Res.

Wu, C. C., Kruse, F., Vasudevarao, M. D., Junker, J. P., Zebrowski, D. C., Fischer, K., Noël, E. S., Grün, D., Berezikov, E., Engel, F. B., et al. (2016). Spatially Resolved Genome-wide Transcriptional Profiling Identifies BMP Signaling as Essential Regulator of Zebrafish Cardiomyocyte Regeneration. Dev. Cell.

Wullimann, M. F., Rupp, B., Reichert, H., Wullimann, M. F., Rupp, B. and Reichert, H. 
(1996). The brain of the zebrafish Danio rerio: a neuroanatomical atlas. In Neuroanatomy of the Zebrafish Brain, .

Wunderlich, C., Schober, K., Lange, S. A., Drab, M., Braun-Dullaeus, R. C., Kasper, M., Schwencke, C., Schmeisser, A. and Strasser, R. H. (2006). Disruption of caveolin-1 leads to enhanced nitrosative stress and severe systolic and diastolic heart failure. Biochem. Biophys. Res. Commun.

Xiao, C., Gao, L., Hou, Y., Xu, C., Chang, N., Wang, F., Hu, K., He, A., Luo, Y., Wang, J., et al. (2016). Chromatin-remodelling factor Brg1 regulates myocardial proliferation and regeneration in zebrafish. Nat. Commun.

Xin, M., Kim, Y., Sutherland, L. B., Murakami, M., Qi, X., McAnally, J., Porrello, E. R., Mahmoud, A. I., Tan, W., Shelton, J. M., et al. (2013). Hippo pathway effector Yap promotes cardiac regeneration. Proc. Natl. Acad. Sci. U. S. A.

Xu, S., Webb, S. E., Lau, T. C. K. and Cheng, S. H. (2018). Matrix metalloproteinases (MMPs) mediate leukocyte recruitment during the inflammatory phase of zebrafish heart regeneration. Sci. Rep.

Xu, S., Xie, F., Tian, L., Manno, S. H., Manno, F. A. M. and Cheng, S. H. (2019). Prolonged neutrophil retention in the wound impairs zebrafish heart regeneration after cryoinjury. Fish Shellfish Immunol.

Yamada, E. (1955). The fine structure of the gall bladder epithelium of the mouse. J. Biophys. Biochem. Cytol.

Yamada, H., Inazumi, T., Tajima, S., Muramatsu, H. and Muramatsu, T. (1997). Stimulation of collagen expression and glycosaminoglycan synthesis by midkine in human skin fibroblasts. Arch. Dermatol. Res.

Yelon, D., Horne, S. A. and Stainier, D. Y. R. (1999). Restricted expression of cardiac myosin genes reveals regulated aspects of heart tube assembly in zebrafish. Dev. Biol.

Yu, J. K., Sarathchandra, P., Chester, A., Yacoub, M., Brand, T. and Butcher, J. T. (2018). Cardiac regeneration following cryoinjury in the adult zebrafish targets a maturation-specific biomechanical remodeling program. Sci. Rep.

Yuan, X., Song, M., Devine, P., Bruneau, B. G., Scott, I. C. and Wilson, M. D. (2018). Heart enhancers with deeply conserved regulatory activity are established early in zebrafish development. Nat. Commun.

Zelenchuk, T. A. and Brusés, J. L. (2011). In Vivo labeling of zebrafish motor neurons using an mnx1 enhancer and Gal4/UAS. Genesis.

Zhang, B., Day, D. S., Ho, J. W., Song, L., Cao, J., Christodoulou, D., Seidman, J. G., Crawford, G. E., Park, P. J. and Pu, W. T. (2013). A dynamic H3K27ac signature identifies VEGFA-stimulated endothelial enhancers and requires EP300 activity. Genome Res.

Zhang, L., Yang, Y., Li, B., Scott, I. C. and Lou, X. (2018). The DEAD-box RNA helicase 
Ddx39ab is essential for myocyte and lens development in zebrafish. Development.

Zhao, Y.-Y., Liu, Y., Stan, R.-V., Fan, L., Gu, Y., Dalton, N., Chu, P.-H., Peterson, K., Ross, J. and Chien, K. R. (2002). Defects in caveolin-1 cause dilated cardiomyopathy and pulmonary hypertension in knockout mice. Proc. Natl. Acad. Sci.

Zhao, S. L., Zhang, Y. J., Li, M. H., Zhang, X. L. and Chen, S. L. (2014). Mesenchymal stem cells with overexpression of midkine enhance cell survival and attenuate cardiac dysfunction in a rat model of myocardial infarction. Stem Cell Res. Ther.

Zhao, L., Ben-Yair, R., Burns, C. E. and Burns, C. G. (2019). Endocardial Notch Signaling Promotes Cardiomyocyte Proliferation in the Regenerating Zebrafish Heart through Wnt Pathway Antagonism. Cell Rep.

Zhu, X., Xiao, C. and Xiong, J.-W. (2018). Epigenetic Regulation of Organ Regeneration in Zebrafish. J. Cardiovasc. Dev. Dis.

Zogbi, C., Saturi de Carvalho, A. E. T., Nakamuta, J. S., Caceres, V. de M., Prando, S., Giorgi, M. C. P., Rochitte, C. E., Meneghetti, J. C. and Krieger, J. E. (2014). Early postnatal rat ventricle resection leads to long-term preserved cardiac function despite tissue hypoperfusion. Physiol. Rep.

Zou, P., Muramatsu, H., Sone, M., Hayashi, H., Nakashima, T. and Muramatsu, T. (2006). Mice doubly deficient in the midkine and pleiotrophin genes exhibit deficits in the expression of $\beta$-tectorin gene and in auditory response. Lab. Investig. 


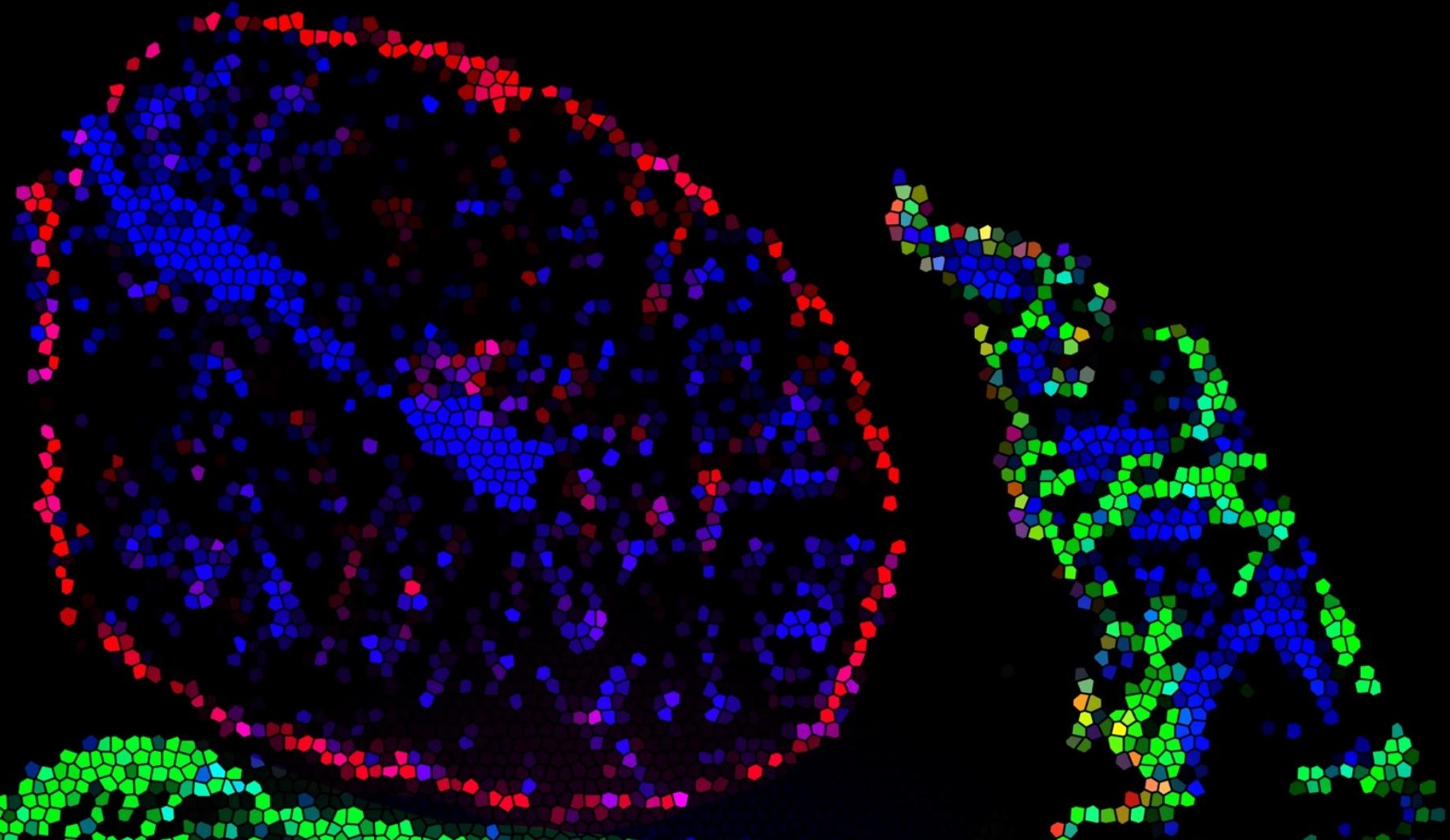

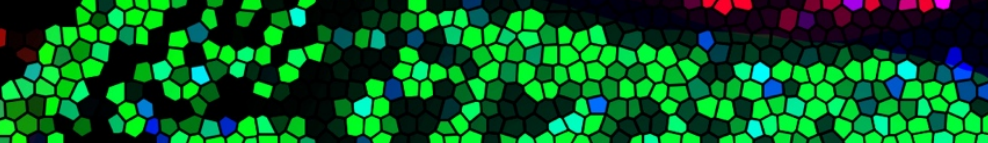

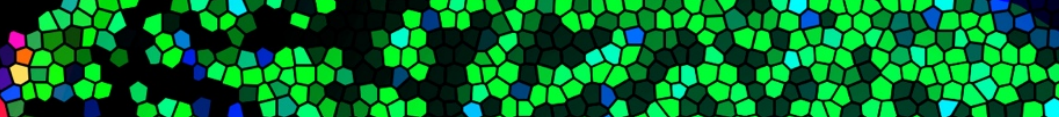
K5:

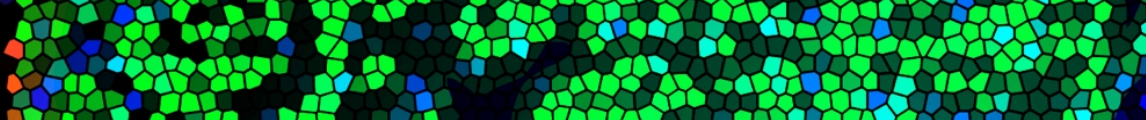

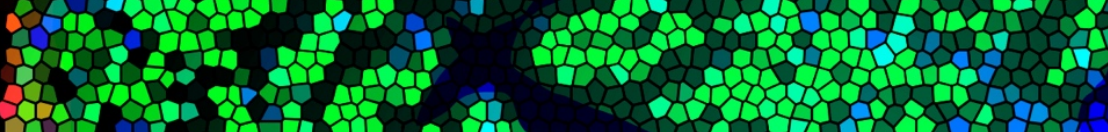

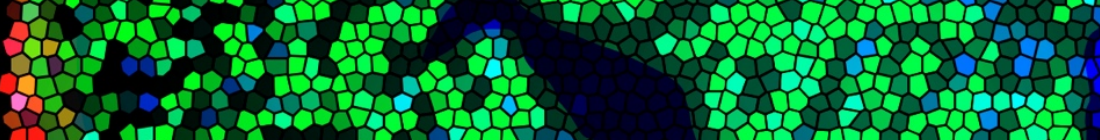

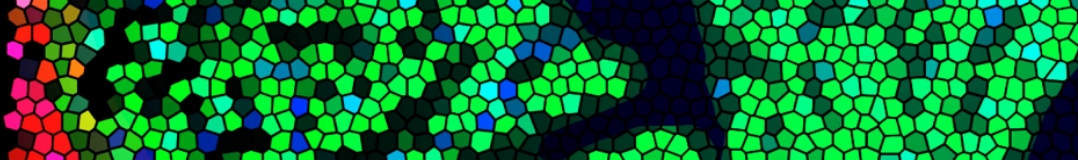

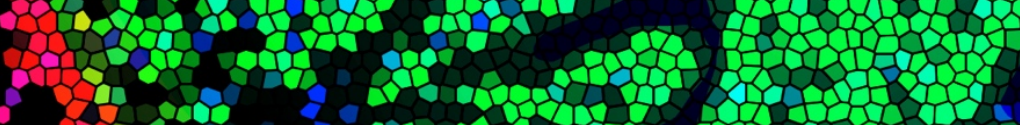

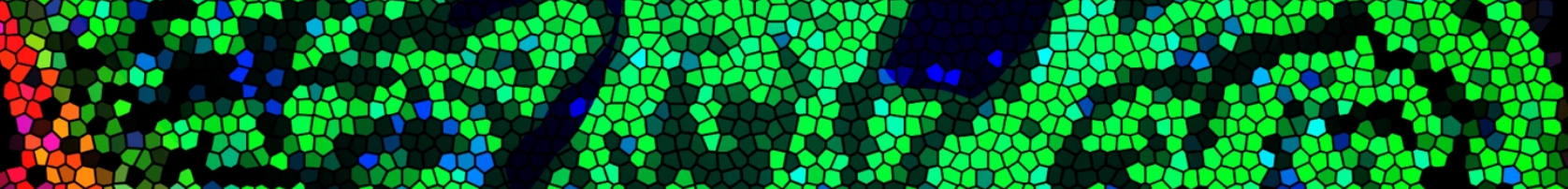

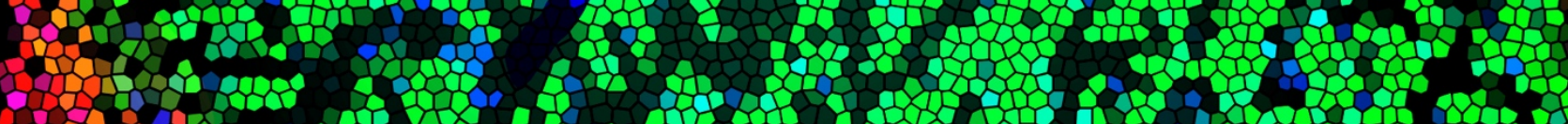
H. \&.35 203030

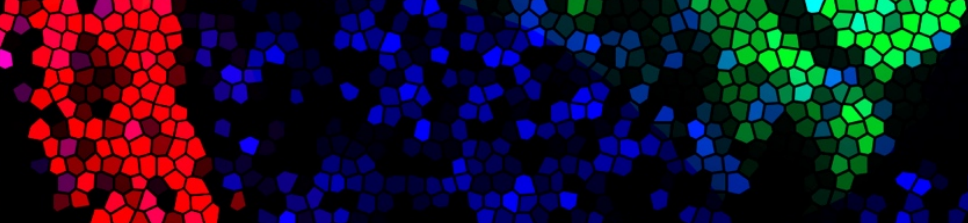

sut Lets 2035 B.82

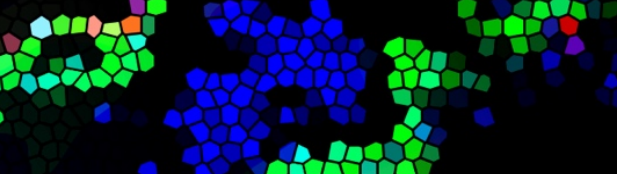

